\title{
ELECTROMAGNETIC FIELD FLUCTUATIONS AND OPTICAL NONLINEARITIES IN METAL-DIELECTRIC COMPOSITES
}

Andrey K. SARYCHEV ${ }^{\mathrm{a}, \mathrm{b}}$, Vladimir M. SHALAEV ${ }^{\mathrm{a}}$

${ }^{a}$ Department of Physics, New Mexico State University, Las Cruces, NM 88003, USA ${ }^{\mathrm{b}}$ Center for Applied Problems of Electrodynamics, 127412, Moscow, Russia

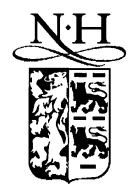

ELSEVIER 


\title{
Electromagnetic field fluctuations and optical nonlinearities in metal-dielectric composites
}

\author{
Andrey K. Sarychev ${ }^{\mathrm{a}, \mathrm{b}}$, Vladimir M. Shalaev ${ }^{\mathrm{a}, *}$ \\ ${ }^{a}$ Department of Physics, New Mexico State University, Las Cruces, NM 88003, USA \\ ${ }^{\mathrm{b}}$ Center for Applied Problems of Electrodynamics, 127412, Moscow, Russia
}

\section{Contents}

1. Introduction

2. Scaling theory of field fluctuations and high-order field moments

2.1. Local field distribution in percolation composites with $\varepsilon_{\mathrm{d}}=-\varepsilon_{\mathrm{m}}^{\prime}$

2.2. High-order moments of local electric fields

2.3. Field fluctuations for the frequencies much below the resonance $\left(\left|\varepsilon_{\mathrm{m}}\right| \gg 1\right)$

3. Numerical and experimental studies of field distributions in two-dimensional percolation composites

3.1. Numerical model

3.2. Field distribution on semicontinuous metal films

4. Anomalous light scattering from semicontinuous metal films

4.1. Rayleigh scattering

4.2. Scaling properties of correlation function
5. Raman scattering

5.1. General formalism

5.2. Raman and hyper-Raman scattering in metal-dielectric composites

6. Giant enhancements of optical nonlinearities in metal-dielectric composites

6.1. Kerr-type optical nonlinearities

6.2. Percolation-enhanced nonlinear scattering from metal-dielectric films

7. Electromagnetic properties of metaldielectric crystals and composites: beyond the quasistatic approximation

7.1. Metal-dielectric composites

7.2. Electromagnetic crystals

7.3. Optical properties of metal semicontinuous films beyond the quasistatic approximation

References

\begin{abstract}
A scaling theory of local field fluctuations and optical nonlinearities is developed for random metaldielectric composites near a percolation threshold. The theory predicts that in the optical and infrared spectral ranges the local fields are very inhomogeneous and consist of sharp peaks representing localized surface plasmons (s.p.). The localization maps the Anderson localization problem described by the random
\end{abstract}

\footnotetext{
* Corresponding author. Fax: + 1-(505)-6461934.

E-mail address: vshalaev@nmsu.edu (V.M. Shalaev).
} 
Hamiltonian with both on- and off-diagonal disorder. The local fields exceed the applied field by several orders of magnitudes resulting in giant enhancements of various optical phenomena. A new numerical method based on the developed theory is suggested. This method is employed to calculate the giant field fluctuations and enhancement of various optical processes in 2D metal-dielectric composites - semicontinuous metal films. The local field fluctuations appear to be highly correlated in space. These fluctuations result in dramatically enhanced Rayleigh and Raman light scattering. The scaling analysis is performed to describe the giant light scattering in a vicinity of the percolation threshold. The developed theory describes quantitatively enhancement of various nonlinear optical processes in percolation composites. It is shown that enhancement depends strongly on whether nonlinear multiphoton scattering includes an act of photon subtraction (annihilation). The magnitudes and spectral dependencies of enhancements in optical processes with photon subtraction, such as Raman and hyper-Raman scattering, Kerr refraction and four-wave mixing, are dramatically different from those processes without photon subtraction, such as sum-frequency and high-harmonic generation. Electromagnetic properties of metal-dielectric crystals and composites beyond the quasistatic approximation are also studied. Equations of macroscopic electromagnetism are presented for these systems. Both linear and nonlinear optical responses are considered in the case of a strong skin effect in metal grains. It is shown that the magnetic field undergoes giant spatial fluctuations. Scaling properties of the local magnetic field and its high-order moments are analyzed. (C) 2000 Elsevier Science B.V. All rights reserved.

PACS: 72.20. - e; 73.20.Mf; 72.15.Gd

Keywords: Composites; Percolation; Optics 


\section{Introduction}

Local electromagnetic field fluctuations and related enhancement of nonlinear optical phenomena in metal-dielectric composites near percolation threshold (percolation composites) have recently become an area of active studies, because of many fundamental problems involved and high potential for various applications. At zero frequency, strong nonlinearity may result in breaking down conducting elements when the electric current exceeds some critical value [1-4]. If the external electric field exceeds some value known as a critical field, a crack spreads over the system. The critical field decreases to zero when the concentration of the conducting component approaches the percolation threshold. This indicates that percolation composites become progressively more responsive to the external field when the percolation threshold is approached. The simplest fuse model can be applied, e.g., for description of fractures in disordered media and related problem of weak tensility of materials in comparison with the strength of the atomic bonds [5]. The tension concentrates around weak points of materials and a crack spreads out starting from these weak points.

Another example of unusual nonlinear behavior has been observed recently for the AC and DC conductivities in a percolation mixture of carbon particles embedded in the wax matrix [6]. In this case, neither carbon particles nor wax matrix have any nonlinearity in their conductivities; nevertheless, the conductivity of a macroscopic composite sample increases twice when the applied voltage increases by few volts. Such a strong nonlinear response can be attributed to the quantum tunneling between conducting (carbon) particles, which is a distinguished feature of the electric transport in composites near the percolation threshold [7]. The current and electric field are concentrated in few "hot" junctions and make it possible to change their conductances under action of the high local fields, whereas the external field is relatively small. In general, percolation systems are very sensitive to the external electric field since their transport and optical properties are determined by rather sparse network of conducting channels, and the field concentrates in the weak points of the channels. Therefore, composite materials should have much larger nonlinear susceptibilities at zero and finite frequencies than those of its constitutes.

The distinct feature of percolation composites, which amplifies nonlinearities of its components, has been recognized very early [4,5,8-11], and nonlinear conductivities and susceptibilities have been intensively studied during the last decade (see, for example, [12-17,140]).

In this review article we consider relatively weak nonlinearities when conductivity $\sigma(E)$ can be expanded in the power series of the applied electric field $E$, and the leading term, i.e., the linear conductivity $\sigma^{(1)}$, is much larger than others. This situation is typical for various nonlinearities in the optical and infrared spectral ranges considered here. Even weak nonlinearities lead to qualitatively new physical effects. For example, generation of higher harmonics can be strongly enhanced in percolation composites and bistable behavior of the effective conductivity can occur when the conductivity switches between two stable values [18]. We note that the "languages" of nonlinear currents/conductivities and nonlinear polarizations/susceptibilities (or dielectric constants) are completely equivalent and they will be used here interchangeably.

The local field fluctuations can be strongly enhanced in the optical and infrared spectral ranges for a composite material containing metal particles that are characterized by the dielectric constant with negative real and small imaginary parts. Then, the enhancement is due to the surface plasmon resonance in metallic granules and their clusters $[12,14,19,20]$. The strong fluctuations of the local 
electric field lead to enhancement of various nonlinear effects. Nonlinear percolation composites are potentially of great practical importance [21] as media with intensity-dependent dielectric functions and, in particular, as nonlinear filters and optical bistable elements. The optical response of nonlinear composites can be tuned, for example, by controlling the volume fraction and morphology of constitutes.

In Refs. [10,22-24] nonlinear optical properties of fractal aggregates of metal particles have been studied. The main result is that the aggregation of initially isolated particles into fractals results in huge enhancements of the nonlinear responses within the spectral range of the cluster plasmon resonances. Typical size $a \sim 10 \mathrm{~nm}$ of metal particles in fractal clusters is much smaller than the wavelength $\lambda>300 \mathrm{~nm}$ in the optical and infrared spectral ranges. The average density of particles in fractals is much smaller than in bulk materials and tends to zero with increasing the fractal size. With this simplifications, it is possible to consider each particle as an elementary dipole and introduce corresponding interaction operator. Then the problem of the optical response of metal fractals reduces to diagonalizing the interaction operator for the dipoles induced by light. If the number of metal particles in a fractal aggregate is not very large the diagonalization of the interaction operator can be done numerically and thus the local electric field can be calculated. Local fields fluctuations in metal fractals were studied numerically, for example, in [25-27]. It has been found that the areas of large field fluctuations are localized in different small parts of a fractal that change with the wavelength.

The prediction of large enhancements of optical nonlinearities in metal fractals has been verified experimentally for the example of degenerate four-wave mixing and nonlinear refraction and absorption [22]. Aggregation of initially isolated silver particles into fractal clusters in these experiments led to a $10^{6}$-fold enhancement of the efficiency of the nonlinear four-wave process and $\sim 10^{3}$ enhanced nonlinear refraction and absorption. The localized and strongly fluctuating local fields in fractals were imaged by means of the near-field scanning optical microscopy (NSOM) in [27]. Similar pattern for the field distribution was obtained for self-affine thin films [28] that have fractal structure of the surface, with different scaling properties in the plane of the film and normal to it.

Enhanced optical processes in composites with layered and other structures were studied by Sipe, Boyd and their co-workers [29] both theoretically and experimentally. The theoretical treatment of nonlinear effects in composite with parallel slabs microstructure can be performed analytically due to the rather simple geometry of the system (see also [18]). Nonlinear susceptibilities of some hierarchical structures and periodic composites with shell structure were considered in [30] and [31], respectively.

In spite of big efforts and some achievements, outlined above, the local field distribution and corresponding nonlinearities were, till very recently, poorly known for percolation metal-dielectric composites, in the most interesting spectral range where the plasmon resonances occur in metal grains. When a small volume concentration $p \ll 1$ of the nonlinear material is embedded in a linear host the effective nonlinear response of the whole composite can be calculated explicitly [32,33]. As one could expect the nonlinearities are enhanced at the frequency $\omega_{\mathrm{r}}$ corresponding to the plasmon resonance of a single metal grain. Numerical calculations [34] for a finite concentration $p$ also give a considerable enhancement in the narrow frequency range around $\omega_{\mathrm{r}}$. These calculations also show that the system sizes tractable for the known numerical methods [35] are not enough to make quantitative conclusions about the nonlinear properties for the frequencies $\omega$ close to the 
resonance frequency $\omega_{\mathrm{r}}$. Thus the system size $L$ equals to $L=10$ in calculations of Ref. [34], whereas the local field fluctuations have typically much larger spatial separation $\xi_{\mathrm{e}}$ for the frequencies $\omega<\omega_{\mathrm{r}}[15,36-48]$. Then the system size $L$ is an artificial damping factor that cuts off all field fluctuations with $\xi_{\mathrm{e}}>L$ and results in the corresponding decrease of the nonlinearities.

To avoid direct numerical calculations, the effective medium theory [49] that has the virtue of mathematical and conceptual simplicity, was extended for the nonlinear response of percolation composites $[12,13,50-55]$ and fractal clusters [56]. For linear problems, predictions of the effective medium theory are usually sensible physically and offer quick insight into problems that are difficult to attack by other means [12]. The effective medium theory, however, has disadvantages typical for all mean-field theories, namely, it diminishes fluctuations in a system. For example, it assumes that local electric fields are the same in the volume occupied by each component of a composite. The electric fields in different components are determined self-consistently.

For the static case the results of the last modification of the nonlinear effective medium theory $[54,55]$ are in best agreement with comprehensive computer simulations performed for a twodimensional (2D) percolation composite $[52,54,55]$. The original approach that combines the effective medium theory and spectral representation [12,57] has been developed in Refs. [16,140]. In spite of this success, application of any kind nonlinear effective medium theories is rather questionable for the frequency range corresponding to the plasmon resonances in metal grains. The first computer $[15,36-38,40-45,47]$ and experimental results $[39,46,58]$ for the field distribution in percolation composites show that the local field distributions contain sharp peaks with distances between them much larger than the metal grain size. This pattern agrees qualitatively with numerical calculations and experimental results for metal fractals $[25,27,59,60]$ and self-affine films [28]. Therefore, the local electric field by no means can be considered as the same in all metal grains of the composite. Then the main assumption of the effective medium theory fails for the frequency range corresponding to the plasmon resonance in the films.

A new theory of electromagnetic field distribution and nonlinear optical processes in metaldielectric composites has been developed recently [15,36-48]. The new approach is based on a percolation theory and the fact that the problem of optical excitations in percolation composites mathematically maps the Anderson transition problem. The theory predicts localization of surface plasmons (s.p.) in percolation composites and describes in detail the localization pattern. It is shown that the s.p. eigenstates are localized on the scale much smaller than the wavelength of an incident light. The s.p. eigenstates with eigenvalues close to zero (resonant modes) are excited most efficiently by the external field. Since the eigenstates are localized and only a small portion of them are excited by the incident beam, the overlapping of the eigenstates can typically be neglected and that significantly simplifies theoretical considerations and allows one to obtain relatively simple expressions for enhancements of linear and nonlinear optical responses. It is important to stress that the s.p. localization length is much smaller than the light wavelength; in that sense, the predicted sub-wavelength localization of the s.p. differs from the well-known localization of light due to strong scattering in a random homogeneous medium [61].

We also note that the developed scaling theory of optical nonlinearities in percolation composites opens new means to study the classical Anderson problem, taking advantage of unique characteristics of laser radiation, namely, its coherence and high intensity. For example, the new theory predicts that at percolation there is a minimum in nonlinear optical responses of 
metal-dielectric composites, the fact that follows from the Anderson localization of s.p. modes and can be studied and verified in laser experiments.

The rest of the paper is organized as follows. In Section 2, we consider local fields and their high-order moment distributions in percolation composites. We also show there that the field distribution maps the Anderson localization problem in quantum mechanics and employ this fact to describe in detail a localization pattern of s.p. modes. The mapping and scaling arguments are used to obtain the field high-order moments and their dependencies on the frequency of an incident wave and metal concentration, for arbitrary optical nonlinearity. Our numerical approach and computer results for the local fields are described in Section 3. In Section 4 we consider light scattering (Rayleigh scattering) from two-dimensional (2D) metal-dielectric composites also known as semicontinuous metal films. It is generally accepted that fluctuations are especially strong in 2D systems. Therefore, we speculate that enhancement of different optical effects due to the local field fluctuations are especially large in semicontinuous metal films. In Section 5 we present self-consistent, general theory of Raman scattering in inhomogeneous media. The results of this theory are used to find surface-enhanced Raman scattering in semicontinuous metal films. Hyper-Raman scattering is also discussed in this section. In Section 6, we calculate enhancement factors for a number of nonlinear optical processes, including Kerr-type nonlinear refraction and absorption and $n$th harmonic generation. We show that most of the enhancement originates from strongly localized nanometer-scale areas, where the local electric field has its maxima. Enhancements in these "hot zones" are giant and exceed a "background" nonlinear signal by many orders of magnitude. In Section 7 we generalize our approach for the case when the local electromagnetic field cannot be considered as a potential field (even on a "microscopical" scale), i.e., when the skin effect is strong. Two different classes of metal-dielectric systems will be analyzed, percolation composites and artificial electromagnetic crystals three-dimensional periodic metal structures $[62,63]$. We show that the electromagnetic properties of the composites and electromagnetic crystals can be understood in terms of effective dielectric constant and magnetic permeability as soon as the wavelength of the incident wave is much larger than an intrinsic spatial scale of the system. The most interesting effects we expect in the limit of wavelength vanishing inside the metal, that is in the limit of the strong skin effect. Thus effective dielectric constant becomes negative in some metaldielectric crystals.

\section{Scaling theory of field fluctuations and high-order field moments}

In metal-dielectric percolation composites the effective DC conductivity $\sigma_{\mathrm{e}}$ decreases with decreasing the volume concentration of metal component $p$ and vanishes when the concentration $p$ approaches concentration $p_{c}$ known as a percolation threshold $[12,20,64]$. In the vicinity of the percolation threshold $p_{c}$, the effective conductivity $\sigma_{\mathrm{e}}$ is determined by an infinite cluster of percolating (conducting) channels. For concentration $p$ smaller than the percolation threshold $p_{c}$, the effective DC conductivity $\sigma_{\mathrm{e}}=0$, that is the system is a dielectric like. Therefore, metal-insulator transition takes place at $p=p_{c}$. Since the metal-insulator transition associated with percolation represents a geometric phase transition one can anticipate that the current and field fluctuations are scale invariant and large. 
In percolation composites, however, the fluctuation pattern appears to be quite different from that for a second-order transition, where fluctuations are characterized by the long-range correlation, and their relative magnitudes are of the order of unity, at any point of a system $[65,66]$. In contrast, for a DC percolation, local electric fields are concentrated at the edges of large metal clusters so that the field maxima (large fluctuations) are separated by distances of an order of the percolation correlation length $\xi_{\mathrm{p}}$, which diverges when the metal volume concentration $p$ approaches the percolation threshold $p_{c}[34,64,67]$.

We show below that the difference in fluctuations becomes even more striking in the optical spectral range, where the local field peaks have the resonance nature and, therefore, their relative magnitudes can be up to $10^{5}$, for the linear response, and $10^{20}$ and more, for nonlinear responses, with distances between the peaks much larger than the percolation correlation length $\xi_{\mathrm{p}}$.

In the optical and infrared spectral ranges, the surface plasmon resonances play a crucial role in metal-dielectric composites. To get an insight into the high-frequency properties of metals, we first consider a simple model known as a Drude metal that reproduces semi-quantitatively the basic optical properties of a metal. In this approach, the dielectric constant of metal grains can be approximated by the Drude formula

$$
\varepsilon_{\mathrm{m}}(\omega)=\varepsilon_{\mathrm{b}}-\left(\omega_{\mathrm{p}} / \omega\right)^{2} /\left[1+\mathrm{i} \omega_{\tau} / \omega\right],
$$

where $\varepsilon_{\mathrm{b}}$ is the contribution to $\varepsilon_{\mathrm{m}}$ due to the inter-band transitions, $\omega_{\mathrm{p}}$ is the plasma frequency, and $\omega_{\tau}=1 / \tau \ll \omega_{\mathrm{p}}$ is the relaxation rate. In the high-frequency range considered here, losses in metal grains are relatively small, $\omega_{\tau} \ll \omega$. Therefore, the real part $\varepsilon_{\mathrm{m}}^{\prime}$ of the metal dielectric function $\varepsilon_{\mathrm{m}}$ is much larger (in modulus) than the imaginary part $\varepsilon_{\mathrm{m}}^{\prime \prime}\left(\left|\varepsilon_{\mathrm{m}}^{\prime}\right| / \varepsilon_{\mathrm{m}}^{\prime \prime} \cong \omega / \omega_{\tau} \gg 1\right)$, and $\varepsilon_{\mathrm{m}}^{\prime}$ is negative for the frequencies $\omega$ less than the renormalized plasma frequency,

$$
\tilde{\omega}_{\mathrm{p}}=\omega_{\mathrm{p}} / \sqrt{\varepsilon_{\mathrm{b}}} .
$$

Thus, the metal conductivity $\sigma_{\mathrm{m}}=-\mathrm{i} \omega \varepsilon_{\mathrm{m}} / 4 \pi \cong\left(\varepsilon_{\mathrm{b}} \tilde{\omega}_{\mathrm{p}}^{2} / 4 \pi \omega\right)\left[\mathrm{i}\left(1-\omega^{2} / \tilde{\omega}_{\mathrm{p}}^{2}\right)+\omega_{\tau} / \omega\right]$ is characterized by the dominant imaginary part for $\tilde{\omega}_{\mathrm{p}}>\omega \gg \omega_{\tau}$, i.e., it is of inductive character. Therefore, the metal grains can be thought of as inductances $L$, while the dielectric gaps can be represented by capacitances $C$. Then, the percolation composite represents a set of randomly distributed $L$ and $C$ elements. The collective surface plasmons excited by the external field, can be thought of as resonances in different $L-C$ circuits, and the excited surface plasmon eigenstates are seen as giant fluctuations of the local field. The discussion below of the giant field fluctuations is based on the recent works [46-48].

\subsection{Local field distribution in percolation composites with $\varepsilon_{\mathrm{d}}=-\varepsilon_{\mathrm{m}}^{\prime}$}

We suppose that a percolation composite is illuminated by light and consider local optical field distributions. A typical metal grain size $a$ in the percolation nanocomposites is about few nanometers [14] and that is much smaller than the wavelength $\lambda$ of the light in the visible and infrared spectral ranges. When wavelength $\lambda$ is much larger than the particle size $a$ we can introduce potential $\phi(\boldsymbol{r})$ for the local electric field. Then the local current density $\boldsymbol{j}$ can be written as $\boldsymbol{j}(\boldsymbol{r})=\sigma(\boldsymbol{r})\left(-\nabla \phi(\boldsymbol{r})+\boldsymbol{E}_{0}\right)$, where $\boldsymbol{E}_{0}$ is the applied field and $\sigma(\boldsymbol{r})$ is the local conductivity. In the considered quasistatic case the field distribution problem reduces to solution of the Poisson 
equation, representing the current conservation law $\operatorname{div} \boldsymbol{j}=0$, namely

$$
\nabla \cdot\left(\sigma(\boldsymbol{r})\left[-\nabla \phi(\boldsymbol{r})+\boldsymbol{E}_{0}\right]\right)=0,
$$

where the local conductivity $\sigma(\boldsymbol{r})$ takes either $\sigma_{\mathrm{m}}$ or $\sigma_{\mathrm{d}}$ values, for metal and dielectric components, respectively. It is convenient to rewrite Eq. (2.3) in terms of the local dielectric constant $\varepsilon(\boldsymbol{r})=4 \pi \mathrm{i} \sigma(\boldsymbol{r}) / \omega$ as follows:

$$
\nabla \cdot[\varepsilon(\boldsymbol{r}) \nabla \phi(\boldsymbol{r})]=\mathscr{E},
$$

where $\mathscr{E}=\nabla \cdot\left[\varepsilon(\boldsymbol{r}) \boldsymbol{E}_{0}\right]$. The external field $\boldsymbol{E}_{0}$ can be chosen real, while the local potential $\phi(\boldsymbol{r})$ takes complex values since the metal dielectric constant $\varepsilon_{\mathrm{m}}$ is complex $\varepsilon_{\mathrm{m}}=\varepsilon_{\mathrm{m}}^{\prime}+\mathrm{i} \varepsilon_{\mathrm{m}}^{\prime \prime}$ in the optical and infrared spectral ranges. Because of difficulties in finding solution to the Poisson Eq. (2.3) or (2.4), a great deal of use has been made of the tight binding model in which metal and dielectric particles are represented by metal and dielectric bonds of a cubic lattice. After such discretization, Eq. (2.4) acquires the form of Kirchhoff's equations defined on a cubic lattice [12]. We write the Kirchhoff's equations in terms of the local dielectric constant and assume that the external electric field $\boldsymbol{E}_{0}$ is directed along " $z$ " axis. Thus we obtain the following set of equations:

$$
\sum_{j} \varepsilon_{i j}\left(\phi_{j}-\phi_{i}\right)=\sum_{j} \varepsilon_{i j} E_{i j},
$$

where $\phi_{i}$ and $\phi_{j}$ are the electric potentials determined at the sites of the cubic lattice and the summation is over the nearest neighbors of the site $i$. The electromotive force (EMF) $E_{i j}$ takes value $E_{0} a_{0}$, for the bond $\langle i j\rangle$ in the positive $z$ direction (where $a_{0}$ is the spatial period of the cubic lattice) and $-E_{0} a_{0}$, for the bond $\langle i j\rangle$ in the $-z$ direction; $E_{k j}=0$ for the other four bonds at the site $i$. Thus the composite is modeled by a resistor-capacitor-inductor network represented by Kirchhoff's equations (2.5). The EMF forces $E_{i j}$ represent the external electric field applied to the system.

In transition from the continuous medium described by Eq. (2.3) to the random network described by Eq. (2.5) we suppose, as usual $[12,20,64]$, that bond permittivities $\varepsilon_{i j}$ are statistically independent and set $a_{0}$ equal to the metal grain size, $a_{0}=a$. In the considered case of two component metal-dielectric random composite, the permittivities $\varepsilon_{i j}$ take values $\varepsilon_{\mathrm{m}}$ and $\varepsilon_{\mathrm{d}}$, with probabilities $p$ and $1-p$, respectively. Assuming that the bond permittivities $\varepsilon_{i j}$ in Eq. (2.5) are statistically independent, we considerably simplify computer simulations as well as analytical consideration of local optical fields in the composite. We note that important critical properties are universal, i.e. they are independent of details of a model, e.g., of possible correlations of permittivities $\varepsilon_{i j}$ in different bonds.

For further consideration we assume that the cubic lattice has a very large but finite number of sites $N$ and rewrite Eq. (2.5) in matrix form with the "Hamiltonian" $\hat{H}[46-48]$ defined in terms of the local dielectric constants,

$$
\hat{H} \phi=\mathscr{E},
$$

where $\phi$ is a vector of the local potentials $\phi=\left\{\phi_{1}, \phi_{2}, \ldots, \phi_{N}\right\}$ determined in all $N$ sites of the lattice, vector $\mathscr{E}$ equals to $\mathscr{E}_{i}=\sum_{j} \varepsilon_{i j} E_{i j}$, as it follows from Eq. (2.5). The Hamiltonian $\hat{H}$ is an $N \times N$ matrix that has off-diagonal elements $H_{i j}=-\varepsilon_{i j}$ and diagonal elements defined as $H_{i i}=\sum_{j} \varepsilon_{i j}$, 
where $j$ refers to the nearest neighbors of site $i$. The off-diagonal elements $H_{i j}$ take values $\varepsilon_{\mathrm{d}}>0$ and $\varepsilon_{\mathrm{m}}=(-1+\mathrm{i} \kappa)\left|\varepsilon_{\mathrm{m}}^{\prime}\right|$ with probability $p$ and $1-p$, respectively. The loss factor $\kappa=\varepsilon_{\mathrm{m}}^{\prime \prime} /\left|\varepsilon_{\mathrm{m}}^{\prime}\right|$ is small, $\kappa \ll 1$. The diagonal elements $H_{i i}$ are distributed between $2 d \varepsilon_{\mathrm{m}}$ and $2 d \varepsilon_{\mathrm{d}}$, where $d$ is the dimensionality of the space ( $2 d$ is the number of the nearest neighbors in $d$ dimensional cubic lattice).

It is convenient to represent the Hamiltonian $\hat{H}$ as a sum of two Hermitian Hamiltonians $\hat{H}=\hat{H}^{\prime}+i \kappa \hat{H}^{\prime \prime}$, where the term i $\kappa \hat{H}^{\prime \prime}(\kappa \ll 1)$ represents losses in the system. The Hamiltonian $\hat{H}^{\prime}$ formally coincides with the Hamiltonian of the problem of metal-insulator transition (Anderson transition) in quantum systems [68-71]. More specifically, the Hamiltonian $\hat{H}^{\prime}$ maps the quantum-mechanical Hamiltonian for the Anderson transition problem with both on- and off-diagonal correlated disorder. Since the off-diagonal matrix elements in $\hat{H}^{\prime}$ have different signs, the Hamiltonian is similar to the so-called gauge-invariant model. This model, in turn, is a simple version of the random flux model, which represents a quantum system with random magnetic field [68] (see also recent numerical studies [72-74]). Hereafter, we refer to operator $\hat{H}^{\prime}$ as to Kirchhoff's Hamiltonian (KH).

Thus, the problem of the field distribution in the system, i.e., the problem of finding solution to Kirchhoff's Eqs. (2.5) or (2.6), becomes the eigenfunction problem for the $\mathrm{KH}, \hat{H}^{\prime} \Psi_{n}=\Lambda_{n} \Psi_{n}$, whereas the losses can be treated as perturbation.

Since the real part $\varepsilon_{\mathrm{m}}^{\prime}$ of metal dielectric function $\varepsilon_{\mathrm{m}}$ is negative, $\varepsilon_{\mathrm{m}}^{\prime}<0$, and the permittivity of dielectric host is positive, $\varepsilon_{\mathrm{d}}>0$, the manifold of the $\mathrm{KH}$ eigenvalues $\Lambda_{n}$ contains eigenvalues which have the real parts equal (or close) to zero. Then eigenstates $\Psi_{n}$ that correspond to eigenvalues $\left|\Lambda_{n}\right| \ll\left|\varepsilon_{\mathrm{m}}\right|,\left|\varepsilon_{\mathrm{d}}\right|$ are strongly excited by the external field and seen as giant field fluctuations, representing the resonant s.p. modes. If we assume that the eigenstates excited by the external field are localized, they should look like local field peaks. The average distance between the field peaks can be estimated as $a(N / n)^{1 / d}$, where $n$ is the number of the KH eigenstates excited by the external field and $N$ is the total number of the eigenstates.

Now we consider in more detail the behavior of the eigenfunctions $\Psi_{n}$ of the HK $\hat{H}^{\prime}$, in the special case when $\varepsilon_{\mathrm{m}}^{\prime}=-\varepsilon_{\mathrm{d}}$, corresponding to the plasmon resonance of individual particles in a $2 \mathrm{D}$ system. Since a solution to Eq. (2.5) does not change when multiplying $\varepsilon_{\mathrm{m}}$ and $\varepsilon_{\mathrm{d}}$ by the same factor, we can normalize the system and set $\varepsilon_{\mathrm{d}}=-\varepsilon_{\mathrm{m}}=1$. We also suppose for simplicity that the metal concentration $p=0.5$.

According to the one-parameter scaling theory the eigenstates $\Psi_{n}$ are all localized for the $2 \mathrm{D}$ case (see, however, discussion in [71,75]). On the other hand, it was shown in computer simulations [76] that there is a transition from chaotic [77,78] to localized eigenstates for the 2D Anderson problem [76], with an intermediate crossover region. We consider first the case when metal concentration $p$ is equal to the percolation threshold $p_{c}=1 / 2$ for the $2 \mathrm{D}$ bond percolation problem. Then the on-diagonal disorder in the KH $\hat{H}^{\prime}$ is characterized by $\left\langle H_{i i}^{\prime}\right\rangle=0,\left\langle H_{i i}^{\prime}{ }^{2}\right\rangle=4$ that corresponds to the chaos-localization transition [76]. The KH has also strong off-diagonal disorder, $\left\langle H_{i j}^{\prime}\right\rangle=0(i \neq j)$, which favors localization [72,73]. Our conjecture is that eigenstates $\Psi_{n}$ are localized for all $\Lambda_{n}$ in the $2 \mathrm{D}$ system. (We cannot rule out a possibility of inhomogeneous localization, similar to that obtained for fractals [25], or the power-law localization $[68,79]$; note, however, that these possibilities are in strong disagreement with the one-parameter scaling theory.)

In the considered case of $\varepsilon_{\mathrm{d}}=-\varepsilon_{\mathrm{m}}=1$ and $p=1 / 2$, all parameters in the KH $\hat{H}^{\prime}$ are of the order of unity and its properties do not change under the transformation $\varepsilon_{\mathrm{d}} \Leftrightarrow \varepsilon_{\mathrm{m}}$. Therefore, the real eigenvalues $\Lambda_{n}$ are distributed symmetrically with respect to zero, in an interval of the order of 
one. The eigenstates with $\Lambda_{n} \approx 0$ are effectively excited by the external field and represent the giant local field fluctuations. When metal concentration $p$ decreases (increases), the eigenstates with $\Lambda_{n} \approx 0$ are shifted from the center of the distribution toward its lower (upper) edge, which typically favors localization. Because of this, we assume that the eigenstates, or at least those with $\Lambda_{n} \approx 0$, are localized, for all metal concentrations $p$ in the $2 \mathrm{D}$ case.

Despite the great effort and all the progress made, the Anderson transition is not yet fully understood in the $3 \mathrm{D}$ case and very little is known about the eigenfunctions of the Anderson Hamiltonian, even in the case of a diagonal disorder only [68-71,80]. We mention here recent computer simulations [74] for a 3D system similar to our system with $\varepsilon_{\mathrm{d}}=-\varepsilon_{\mathrm{m}}=1, p=1 / 2$. The authors of [74] investigate the Anderson problem with diagonal matrix elements $w_{i i}$ distributed uniformly around zero $-w_{0} / 2 \leq w_{i i} \leq w_{0} / 2$ and off-diagonal elements $t_{i j}=\exp \left(\mathrm{i} \phi_{i j}\right)$, with phases $\phi_{i j}$ also distributed uniformly $0 \leq \phi_{i j} \leq 2 \pi$. It was found that in the center of the band, the states are localized for the disorder $w_{0}>w_{\mathrm{c}}=18.8$. In our 3D HK $\hat{H}^{\prime}$ Hamiltonian, the diagonal elements are distributed as $-6 \leq H_{i i} \leq 6$ and, therefore, the diagonal disorder is smaller than the above critical disorder $w_{\mathrm{c}}$. On the other hand, our off-diagonal disorder is stronger than in calculations [74]. It is shown [72,73] that even small off-diagonal disorder strongly enforces localization. We conjecture here that the eigenstates corresponding to the eigenvalues $\Lambda_{n} \approx 0$ in the $3 \mathrm{D}$ case are also localized for all $p$.

Suppose we found all eigenvalues $\Lambda_{n}$ and eigenfunctions $\Psi_{n}$ of $\hat{H}^{\prime}$. Then we can express the potential $\phi$ in Eq. (2.6) in terms of the eigenfunctions as $\phi=\sum_{n} A_{n} \Psi_{n}$ and substitute it in Eq. (2.6). Thus we obtain the following equation for coefficients $A_{n}$ :

$$
\left(\mathrm{i} \kappa b_{n}+\Lambda_{n}\right) A_{n}+\mathrm{i} \kappa \sum_{m \neq n}\left(\Psi_{n}\left|\hat{H}^{\prime \prime}\right| \Psi_{m}\right) A_{m}=\mathscr{E}_{n},
$$

where $b_{n}=\left(\Psi_{n}\left|\hat{H}^{\prime \prime}\right| \Psi_{n}\right)$, and $\mathscr{E}_{n}=\left(\Psi_{n} \mid \mathscr{E}\right)$ is a projection of the external field on eigenstate $\Psi_{n}$. (The product of two vectors, e.g., $\Psi_{n}$ and $\mathscr{E}$ is defined here in a usual way, as $\mathscr{E}_{n}=\left(\Psi_{n} \mid \mathscr{E}\right) \equiv \sum_{i} \Psi_{n, i}^{*} \mathscr{E}_{i}$, where the sum is over all lattice sites.) Since all parameters in the real Hamiltonian $\hat{H}^{\prime}$ are of the order of unity, the matrix elements $b_{n}$ are also of the order of unity. We approximate them by some constant $b$, which is about unity. We suggest that eigenstates $\Psi_{n}$ are localized within spatial domains $\xi_{\mathrm{A}}(\Lambda)$, where $\xi_{\mathrm{A}}(\Lambda)$ is the Anderson localization length, which depends on the eigenvalue $\Lambda$. Then, the sum in Eq. (2.7) converges and it can be treated as a small perturbation. In the zeroth approximation,

$$
A_{n}^{(0)}=\mathscr{E}_{n} /\left(\Lambda_{n}+\mathrm{i} \kappa b\right) .
$$

The first-order correction to $A_{n}$ is equal to

$$
A_{n}^{(1)}=-\mathrm{i} \kappa \sum_{m \neq n}\left(\Psi_{n}\left|\hat{H}^{\prime \prime}\right| \Psi_{m}\right) \mathscr{E}_{m} /\left(\Lambda_{m}+\mathrm{i} \kappa b\right) .
$$

For $\kappa \rightarrow 0$, most important eigenstates in this sum are those with $\left|\Lambda_{m}\right| \leq b \kappa$. Since the eigenstates $\Lambda_{n}$ are distributed in the interval of the order of unity the spatial density of the eigenmodes with $\left|\Lambda_{m}\right| \leq b \kappa$ vanishes as $a^{-d} \kappa \rightarrow 0$ at $\kappa \rightarrow 0$. Therefore $A_{n}^{(1)}$ is exponentially small $\left|A_{n}^{(1)}\right| \sim\left|\sum_{m \neq n}\left(\Psi_{n}\left|\hat{H}^{\prime \prime}\right| \Psi_{m}\right) \mathscr{E}_{m} / b_{m}\right| \propto \exp \left\{-\left[a / \xi_{\mathrm{A}}(0)\right] \kappa^{-1 / d}\right\}$ and can be neglected when 
$\kappa \ll\left[a / \xi_{\mathrm{A}}(0)\right]^{d}$. Then, the local potential $\phi$ is equal to $\phi(r)=\sum_{n} A_{n}^{(0)} \Psi_{n}=\sum_{n} \mathscr{E}_{n} \Psi_{n}(r) /\left(\Lambda_{n}+\mathrm{i} \kappa b\right)$ [see Eq. (2.8)] and the fluctuating part of the local field $\boldsymbol{E}_{\mathrm{f}}=-\nabla \phi(\boldsymbol{r})$ is given by

$$
\boldsymbol{E}_{\mathrm{f}}(\boldsymbol{r})=-\sum_{n} \mathscr{E}_{n} \nabla \Psi_{n}(\boldsymbol{r}) /\left(\Lambda_{n}+\mathrm{i} \kappa b\right),
$$

where the nabla operator $\nabla$ is understood as a lattice operator. The average field intensity is as follows:

$$
\left\langle|E|^{2}\right\rangle=\left\langle\left|\boldsymbol{E}_{\mathrm{f}}+\boldsymbol{E}_{0}\right|^{2}\right\rangle=\left|E_{0}\right|^{2}+\left\langle\sum_{n, m} \frac{\mathscr{E}_{n} \mathscr{E}_{m}^{*}\left(\nabla \Psi_{n}(\boldsymbol{r}) \cdot \nabla \Psi_{m}^{*}(\boldsymbol{r})\right)}{\left(\Lambda_{n}+\mathrm{i} \kappa b\right)\left(\Lambda_{m}-\mathrm{i} \kappa b\right)}\right\rangle,
$$

where we took into account that $\left\langle\boldsymbol{E}_{\mathrm{f}}\right\rangle=\left\langle\boldsymbol{E}_{\mathrm{f}}^{*}\right\rangle=0$. We consider now the eigenstates $\Psi_{n}$ with eigenvalues $\Lambda_{n}$ within a small interval $\left|\Lambda_{n}-\Lambda\right| \leq \Delta \Lambda \ll \kappa$ centered at $\Lambda$. These states are denoted as $\Psi_{n}(\Lambda, r)$. Recall that the eigenstates are assumed to be localized so that eigenfunctions $\Psi_{n}(\Lambda, r)$ are well separated in space. The average distance between them, $l$, can be estimated as $l(\Delta \Lambda) \sim a(\rho(\Lambda) \Delta \Lambda)^{-1 / d}$, where

$$
\rho(\Lambda)=a^{d} \sum_{n} \delta\left(\Lambda-\Lambda_{n}\right) / V
$$

is the dimensionless density of states for the $\mathrm{KH} \hat{H}^{\prime}$ and $V$ is the volume of the system. We assume here that the metal concentration $p$ is about one-half so that all quantities in the $\mathrm{KH} \hat{H}^{\prime}$ are about unity and, therefore, the density of states $\rho(\Lambda)$ is also about unity at the center of the spectrum, i.e., at $\Lambda=0$. Then the distance $l(\Delta \Lambda)$ can be arbitrary large for $\Delta \Lambda \rightarrow 0$; we assume, of course, that $l(\Delta \Lambda)$ is still much smaller than the system size, and the total number of eigenstates $\Psi_{n}(\Lambda, r)$ is macroscopically large. When the interstate distance $l(\Delta \Lambda)$ is much larger than the localization length $\xi_{\mathrm{A}}(\Lambda)$ the localized eigenfunctions $\Psi_{n}(\Lambda, r)$ can be characterized by spacial positions of their "centers" $\boldsymbol{r}_{n}$ so that $\Psi_{n}(\Lambda, \boldsymbol{r})=\Psi\left(\Lambda, \boldsymbol{r}-\boldsymbol{r}_{n}\right)$ and Eq. (2.11) acquires the following form:

$$
\left\langle|E|^{2}\right\rangle=\left|E_{0}\right|^{2}+\sum_{\Lambda_{1}, \Lambda_{2}} \frac{\left\langle\sum_{n, m} \mathscr{E}_{n} \mathscr{E}_{m}^{*}\left(\nabla \Psi\left(\Lambda_{1}, \boldsymbol{r}-\boldsymbol{r}_{n}\right) \cdot \nabla \Psi *\left(\Lambda_{2}, \boldsymbol{r}-\boldsymbol{r}_{m}\right)\right)\right\rangle}{\left(\Lambda_{1}+\mathrm{i} \kappa b\right)\left(\Lambda_{2}-\mathrm{i} \kappa b\right)},
$$

where the first sum is over positions of the intervals $\left|\Lambda_{n}-\Lambda_{1}\right|$ and $\left|\Lambda_{m}-\Lambda_{2}\right|$ in the $\Lambda$ space, whereas the sum in the numerator is over spatial positions $\boldsymbol{r}_{n}$ and $\boldsymbol{r}_{m}$ of the eigenfunctions. For each realization of a macroscopically homogeneous random film, the positions $\boldsymbol{r}_{n}$ of eigenfunctions $\Psi\left(\Lambda, \boldsymbol{r}-\boldsymbol{r}_{n}\right)$ take new values that do not correlate with the value of $\Lambda$. Therefore, we can independently average the numerator in the second term of Eq. (2.13) over positions $\boldsymbol{r}_{n}$ and $\boldsymbol{r}_{m}$ of eigenstates $\Psi_{n}$ and $\Psi_{m}$. Taking into account that $\left\langle\nabla \Psi_{n}(r)\right\rangle=0$, we obtain

$$
\left\langle\mathscr{E}_{n} \mathscr{E}_{m}^{*}\left(\nabla \Psi\left(\Lambda_{1}, \boldsymbol{r}-\boldsymbol{r}_{n}\right) \cdot \nabla \Psi *\left(\Lambda_{2}, \boldsymbol{r}-\boldsymbol{r}_{m}\right)\right)\right\rangle \simeq\left\langle\left|\mathscr{E}_{n}\right|^{2}\left|\nabla \Psi\left(\Lambda_{1}, \boldsymbol{r}-\boldsymbol{r}_{n}\right)\right|^{2}\right\rangle \delta_{\Lambda_{1} \Lambda_{2}} \delta_{n m},
$$

where we neglected possible correlations of eigenfunctions from different intervals $\Lambda_{1}$ and $\Lambda_{2}$ since the spatial density of the eigenfunctions excited effectively by the external field is estimated as $a^{-d} \rho(\Lambda) \kappa$, i.e. it vanishes for $\kappa \rightarrow 0$. Substitution of Eq. (2.14) in Eq. (2.11) results in

$$
\left\langle|E|^{2}\right\rangle=\left|E_{0}\right|^{2}+\sum_{\Lambda} \frac{\sum_{n}\left|\mathscr{E}_{n}\right|^{2}\left\langle\left|\nabla \Psi_{n}(\Lambda, r)\right|^{2}\right\rangle}{\Lambda^{2}+(b \kappa)^{2}}
$$


The localized eigenstates are not in general degenerate, so that the eigenfunctions $\Psi_{n}$ can be chosen as real, i.e., $\Psi_{n}=\Psi_{n}^{*}$. Then we can estimate $\left|\mathscr{E}_{n}\right|^{2}=\left|\left(\Psi_{n} \mid \mathscr{E}\right)\right|^{2}=\left|\sum_{i=1}^{N} \Psi_{n, i} \mathscr{E}_{i}\right|^{2}$ in Eq. (2.15) by replacing the sum over all $N$ sites of the system with integration over the system volume $V$, which gives $\left|\mathscr{E}_{n}\right|^{2} \sim a^{-2 d}\left|\int \Psi_{n} \mathscr{E} \mathrm{d} \boldsymbol{r}\right|^{2}$. Using Eqs. (2.5) and (2.4), we find

$$
\left|\mathscr{E}_{n}\right|^{2} \sim a^{4-2 d}\left|\int \Psi_{n}\left(\boldsymbol{E}_{0} \cdot \nabla \varepsilon\right) \mathrm{d} \boldsymbol{r}\right|^{2}=a^{4-2 d}\left|\int \varepsilon\left(\boldsymbol{E}_{0} \cdot \nabla \Psi_{n}\right) \mathrm{d} \boldsymbol{r}\right|^{2},
$$

where to obtain the last relation we integrated by parts and took into account that the eigenstates $\Psi_{n}$ are localized within the localization length $\xi_{\mathrm{A}}(\Lambda)$. Since the local dielectric constants $|\varepsilon|$ are of the order of unity, $|\varepsilon| \sim 1$, and the spatial derivative $\nabla \Psi_{n}$ is estimated as $\Psi_{n} / \xi_{\mathrm{A}}(\Lambda)$ in Eq. (2.16), we find

$$
\left|\mathscr{E}_{n}\right|^{2} \sim \frac{\left|E_{0}\right|^{2} a^{4}}{a^{2 d} \xi_{A}^{2}(\Lambda)}\left|\int \Psi_{n}(\boldsymbol{r}) \mathrm{d} \boldsymbol{r}\right|^{2} \sim \frac{\left|E_{0}\right|^{2} a^{4}}{\xi_{\mathrm{A}}^{2}(\Lambda)}\left|\sum_{i=1}^{N} \Psi_{n, i}\right|^{2},
$$

where we returned to summation over sites of the tight binding model. Because the eigenfunctions $\Psi_{n}$ are normalized to unity, i.e., $\left\langle\Psi_{n} \mid \Psi_{n}\right\rangle=\sum_{i=1}^{N}\left|\Psi_{n, i}\right|^{2}=1$ and localized within $\xi_{\mathrm{A}}(\Lambda)$ we estimate them as $\Psi_{n, i} \sim\left[\xi_{\mathrm{A}}(\Lambda) / a\right]^{-d / 2}$ in the localization domain. Substituting this estimate in Eq. (2.17) we obtain

$$
\left|\mathscr{E}_{n}\right|^{2} \sim\left|E_{0}\right|^{2} a^{2}\left[\xi_{\mathrm{A}}(\Lambda) / a\right]^{d-2} .
$$

In a similar way we can estimate the average spatial derivative in the numerator of Eq. (2.15),

$$
\left\langle\left|\nabla \Psi_{n}(\Lambda, \boldsymbol{r})\right|^{2}\right\rangle \sim \xi_{\mathrm{A}}^{-2}(\Lambda)\left\langle\left|\Psi_{n}(\Lambda, \boldsymbol{r})\right|^{2}\right\rangle \sim \xi_{\mathrm{A}}^{-2}(\Lambda) N^{-1} \sum_{i=1}^{N}\left|\Psi_{n, i}\right|^{2} \sim \xi_{\mathrm{A}}^{-2}(\Lambda) / N,
$$

where $N=V / a^{d}$ is the total number of sites. Now we use the estimates (2.18) and (2.19) and rewrite the numerator of Eq. (2.15) as

$$
\sum_{n}\left|\mathscr{E}_{n}\right|^{2}\left\langle\left|\nabla \Psi_{n}(\Lambda, r)\right|^{2}\right\rangle \sim \frac{1}{N} \sum_{n}\left|E_{0}\right|^{2}\left[\xi_{\mathrm{A}}(\Lambda) / a\right]^{d-4} \sim\left|E_{0}\right|^{2}\left[\xi_{\mathrm{A}}(\Lambda) / a\right]^{d-4} \rho(\Lambda) \Delta \Lambda,
$$

where we took into account the fact that the total number of the eigenstates within interval $\Delta \Lambda$ is equal to $N \rho(\Lambda) \Delta \Lambda$. By substituting (2.20) in Eq. (2.15) and replacing the summation by integration over $\Lambda$, we obtain the following estimate for the field intensity:

$$
\left\langle|E|^{2}\right\rangle \sim\left|E_{0}\right|^{2}+\left|E_{0}\right|^{2} \int \frac{\rho(\Lambda)\left(a / \xi_{\mathrm{A}}(\Lambda)\right)^{4-d}}{\Lambda^{2}+(b \kappa)^{2}} \mathrm{~d} \Lambda .
$$

Since all matrix elements in $\mathrm{KH} H^{\prime}$ are of the order of unity (in fact, the off-diagonal elements are \pm 1 ), the density of states $\rho(\Lambda)$ and localization length $\xi_{\mathrm{A}}(\Lambda)$ change significantly within an interval of an order of one. In contrast, the denominator in Eq. (2.15) has an essential singularity at $\Lambda= \pm \mathrm{i} b \kappa$. Then the second moment of the local electric field $M_{2} \equiv M_{2,0}=\left\langle|E|^{2}\right\rangle /\left|E_{0}\right|^{2}$ is estimated as

$$
M_{2}^{\star} \sim 1+\rho\left(a / \xi_{\mathrm{A}}\right)^{4-d} \int \frac{1}{\Lambda^{2}+(b \kappa)^{2}} \mathrm{~d} \Lambda \sim \rho\left(a / \xi_{\mathrm{A}}\right)^{4-d} \kappa^{-1} \gg 1
$$


provided that $\kappa \ll \rho\left(a / \xi_{\mathrm{A}}\right)^{4-d}$ (we set $\xi_{\mathrm{A}}(\Lambda=0) \equiv \xi_{\mathrm{A}}, \rho(\Lambda=0) \equiv \rho$ and approximated $b$ by unity). Thus the field distribution, in this case, can be described as a set of the KH eigenstates localized within $\xi_{\mathrm{A}}$, with the field peaks having the amplitudes

$$
E_{\mathrm{m}}^{\star} \sim E_{0} \kappa^{-1}\left(a / \xi_{\mathrm{A}}\right)^{2}
$$

which are separated in distance by the field correlation length

$$
\xi_{\mathrm{e}}^{\star} \sim a(\rho \kappa b)^{-1 / d} \sim a(\rho \kappa)^{-1 / d},
$$

where again we used that $b \sim 1$. All the above speculations leading to Eqs. (2.22)-(2.24) hold when the field correlation length $\xi_{\mathrm{e}}^{\star}$ is much larger than the Anderson localization length, i.e., $\xi_{\mathrm{e}}^{\star} \gg \xi_{\mathrm{A}}$. This condition is fulfilled in the limit of small losses when $\kappa \rightarrow 0$.

Note that hereafter by the superscript ${ }^{\star}$ we mark the fields and spatial scales that are given for the special case $-\varepsilon_{\mathrm{m}}=\varepsilon_{\mathrm{d}}=1$ considered here (this sign ${ }^{\star}$, of course, should not be confused with the complex conjugation, denoted by $*$ ). Using the scale renormalization described in Section 2.3, we will see how these quantities are transformed when $\left|\varepsilon_{\mathrm{m}} / \varepsilon_{\mathrm{d}}\right| \gg 1$, i.e., in the long wavelength part of the spectrum. Note also that, for $\xi_{\mathrm{A}}$ and $\rho$ we omit the ${ }^{\star}$ sign in order to avoid complicated notations; it is implied that their values are always taken at $-\varepsilon_{\mathrm{m}}=\varepsilon_{\mathrm{d}}=1$, even if the case of $\left|\varepsilon_{\mathrm{m}} / \varepsilon_{\mathrm{d}}\right| \gg 1$ is considered.

In the above estimates we supposed that the localization length $\xi_{\mathrm{A}}$ is proportional to the eigenstate "size". This assumption might not be exact for the Anderson system, in general (e.g., see discussion in [68]), but it is confirmed well by numerical calculations (see Figs. 1 and 2) for the case of 2D percolation composites.

Above we assumed that metal concentration $p$ is about one-half, which corresponds to the percolation threshold for $d=2$. The derivation of Eqs. (2.21) and (2.22) was based on the assumption that the density of states $\rho(\Lambda)$ is finite and about unity for $\Lambda=0$. This assumption, however, is violated for small metal concentration $p$, when the eigenvalue distribution shifts to the positive side of $\Lambda$ so that the eigenstates with $\Lambda \approx 0$ are shifted to the lower edge of the distribution. Then, the density of states $\rho$ in Eq. (2.22) becomes a function of the metal concentration $p$. In the limit of $p \rightarrow 0$, the number of states effectively excited by the external field is proportional to the number of metal particles. Then the function $\rho(p)$ can be estimated as $\rho(p) \sim p$, for $p \rightarrow 0$. The same consideration holds in the other limit, when a small portion of holes in otherwise continuous film resonate with the external field and the density of states can be estimated as $\rho(p) \sim 1-p$, for $p \rightarrow 1$. When the density of states decreases, localization becomes stronger and we estimate the localization length $\xi_{\mathrm{A}}$ as $\xi_{\mathrm{A}}(\Lambda=0, p \rightarrow 0) \sim \xi_{\mathrm{A}}(\Lambda=0, p \rightarrow 1) \sim a$. This behavior of the field fluctuations is best illustrated in Fig. 1a-g where the results of our computer simulation are shown for $2 \mathrm{D}$ composites - semicontinuous metal films. When the metal concentration $p \rightarrow 0$ or $p \rightarrow 1$ the number of the field maxima decreases while the peaks become progressively sharper. It is seen from Fig. 1 and also follows then from Eq. (2.22) that strong field fluctuations $\left(M_{2}>1\right)$ exist in a metal-dielectric composite with $\varepsilon_{\mathrm{d}}=-\varepsilon_{\mathrm{m}}^{\prime}$ in the wide concentration range

$$
\kappa<p<1-\kappa, \quad \kappa \ll 1 .
$$


(a)
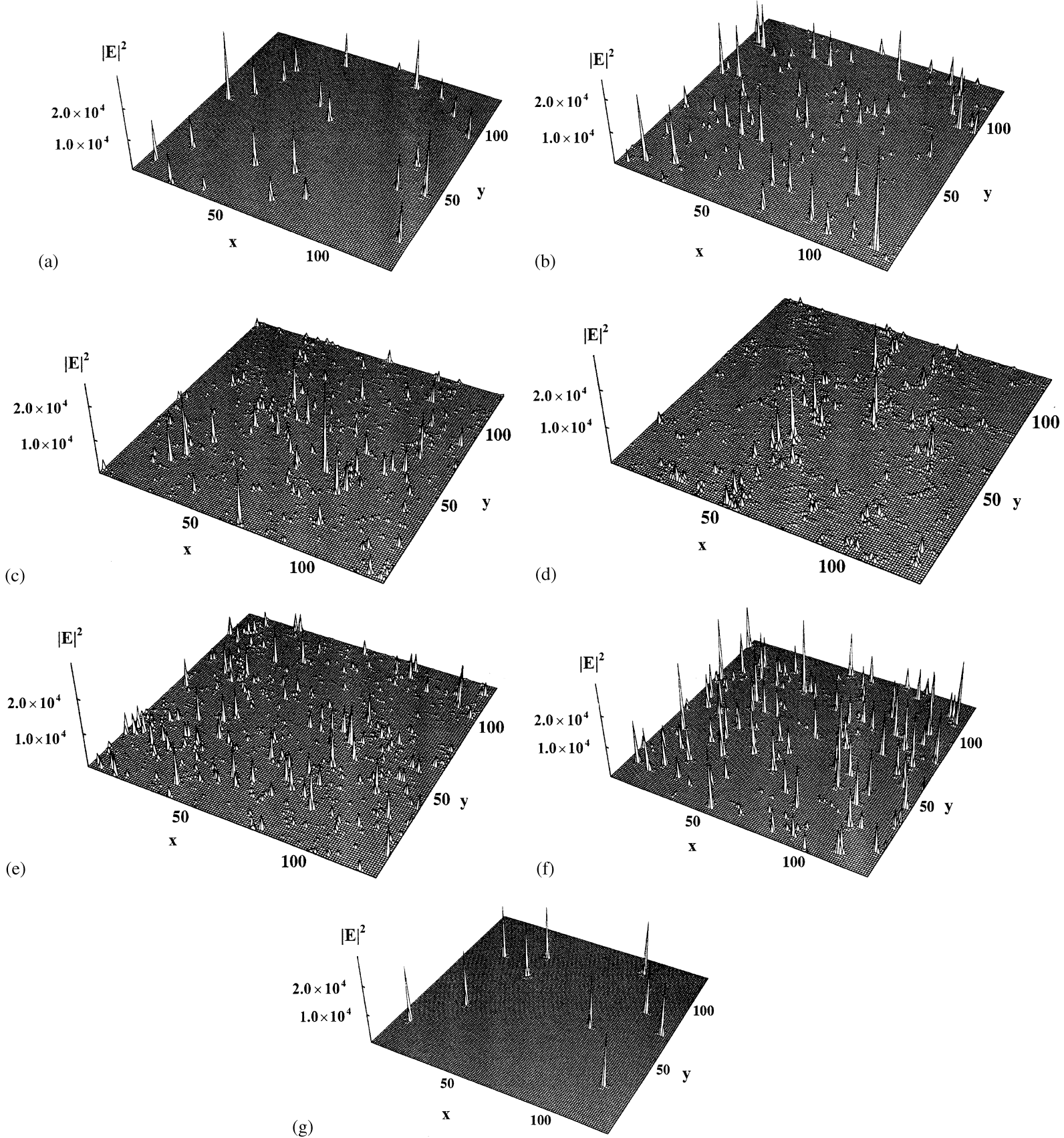

Fig. 1. Distribution of the local field intensities $|E(x, y)|^{2} /\left|E_{0}\right|^{2}$ on a metal (silver) semicontinuous film for $\varepsilon_{\mathrm{m}}^{\prime}=\varepsilon_{\mathrm{d}}=-2.2(\lambda \approx 365 \mathrm{~nm})$ at different metal concentrations, $p$. (a) $p=0.001$, (b) $p=0.01$, (c) $p=0.1$, (d) $p=0.5$, (e) $p=0.9$, (f) $p=0.99$, and (g) $p=0.999$. 

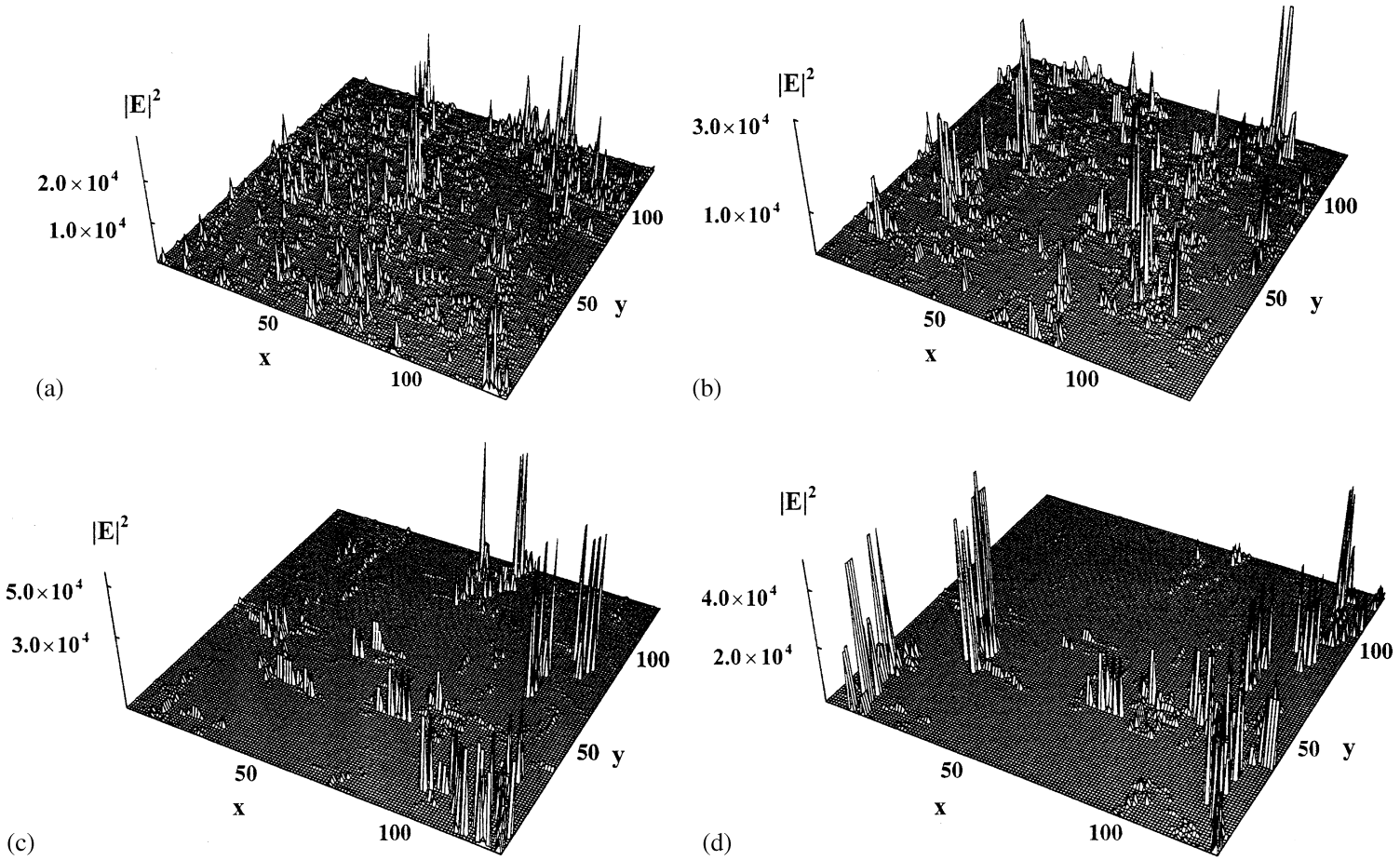

Fig. 2. Distribution of the local field intensities $|E(x, y)|^{2} /\left|E_{0}\right|^{2}$ in a semicontinuous film at the percolation threshold for different wavelengths; (a): $\lambda=0.5 \mu \mathrm{m}$, (b): $\lambda=1.5 \mu \mathrm{m}$, (c): $\lambda=10 \mu \mathrm{m}$, and (d): $\lambda=20 \mu \mathrm{m}$.

Although we estimated the above local fields for the special case of $\varepsilon_{\mathrm{d}}=-\varepsilon_{\mathrm{m}}^{\prime}$ all the above speculations, which are based on the assumption that the eigenstates of $\mathrm{KH}$ are localized, hold in a more general case, when the real part of the metal dielectric constant $\varepsilon_{\mathrm{m}}^{\prime}$ is negative and its absolute value is of the order of $\varepsilon_{\mathrm{d}}$. The important case of the large contrast when $\left|\varepsilon_{\mathrm{m}}\right| \gg \varepsilon_{\mathrm{d}}$ will be considered in Section 2.3.

Note that the above speculations leading to prediction of giant field fluctuations described by Eqs. (2.21) and (2.22), do not require long-range spatial correlations (such, for example, as in fractal structures) in particle positions. The large field fluctuations have been seen in computer simulations, in particular, for the so-called random gas of metal particle [26,24], i.e., for metal particles randomly distributed in space. This, however, is not true when the contrast is large $\left|\varepsilon_{\mathrm{m}}\right| \gg \varepsilon_{\mathrm{d}}$; we show below that in this case the internal structure of a composite becomes crucial.

\subsection{High-order moments of local electric fields}

Now we consider arbitrary high-order field moments defined as

$$
M_{n, m}=\frac{1}{V E_{0}^{m}\left|E_{0}\right|^{n}} \int|E(\boldsymbol{r})|^{n} E^{m}(\boldsymbol{r}) \mathrm{d} \boldsymbol{r}
$$


where, as above, $E_{0} \equiv E^{(0)}$ (both notations are used interchangeably) is the amplitude of the external field and $E(\boldsymbol{r})$ (which is defined so that $E^{2}(\boldsymbol{r}) \equiv \boldsymbol{E}(\boldsymbol{r}) \cdot \boldsymbol{E}(\boldsymbol{r})$ ) is the amplitude of the local field; the integration is over the total volume $V$ of a system. The moments $M_{n, 0}$ we will denote, for simplicity, as

$$
M_{n} \equiv M_{n, 0}=\frac{1}{V\left|E_{0}\right|^{n}} \int|E(\boldsymbol{r})|^{n} \mathrm{~d} \boldsymbol{r}
$$

It is assumed that the volume average in Eqs. (2.26) and (2.27) is equivalent to the ensemble average, i.e., $M_{n, m}=\left\langle|E|^{n} E^{m}\right\rangle / E_{0}^{m}\left|E_{0}\right|^{n}$.

The high-order field moment $M_{2 k, m} \propto\left\langle E^{k+m} E^{* k}\right\rangle$ represents a nonlinear optical process in which in one elementary act $k+m$ photons are added and $k$ photons are subtracted (annihilated) $[81,82]$. This is because the complex conjugated field in the general expression for the nonlinear polarization implies photon subtraction, so that the corresponding frequency enters the nonlinear susceptibility with the sign minus. Enhancement of the Kerr optical nonlinearity $G_{\mathrm{K}}$ is proportional to $M_{2,2}$, third-harmonic generation (THG) enhancement is given by $\left|M_{0,3}\right|^{2}$, and surface-enhanced Raman scattering (SERS) is represented by $M_{4,0}$ (see Sections 4-6). The integrands in Eq. (2.26) for $M_{2,2}$ and $M_{0,3}$, i.e., the local nonlinear field sources $g_{3}=(E(\boldsymbol{r}))^{2} E(\boldsymbol{r}) / E_{0}\left(E_{0}\right)^{2}$ (THG) and $g_{\mathrm{K}}=|E(\boldsymbol{r})|^{2} E^{2}(\boldsymbol{r}) / E_{0}^{2}\left|E_{0}\right|^{2}$ (Kerr optical effect) are shown in Figs. 3 and 4.

We are interested here in the case when $M_{n, m} \gg 1$ which implies that the fluctuating part of the local electric field $\boldsymbol{E}_{\mathrm{f}}$ is much larger than the applied field $\boldsymbol{E}_{0}$. It is suggested, for simplicity, that the applied field is real and $E_{0}=1$. We substitute in Eq. (2.26) the expression for $\boldsymbol{E}_{\mathrm{f}}$ given by Eq. (2.10) and obtain for the moment $M_{2 p, 2 q}$ ( $p$ and $q$ are integers) the following equation:

$$
\begin{aligned}
M_{2 p, 2 q}= & \left\langle\sum_{n_{1}, n_{2}, \ldots, n_{2 p} ; m_{1}, m_{2}, \ldots m_{2 q}}^{N} \frac{\mathscr{E}_{n_{1}} \mathscr{E}_{n_{2}}\left(\nabla \Psi_{n_{1}} \cdot \nabla \Psi_{n_{2}}^{*}\right) \cdots \mathscr{E}_{n_{2 p-1}} \mathscr{E}_{n_{2 p}}\left(\nabla \Psi_{n_{2 p-1}} \cdot \nabla \Psi_{n_{2 p}}^{*}\right)}{\left(\Lambda_{n_{1}}+\mathrm{i} b k\right)\left(\Lambda_{n_{2}}-\mathrm{i} b k\right) \cdots\left(\Lambda_{n_{2 p-1}}+\mathrm{i} b k\right)\left(\Lambda_{n_{2 p}}-\mathrm{i} b k\right)}\right. \\
& \left.\times \frac{\mathscr{E}_{m_{1}} \mathscr{E}_{m_{2}}\left(\nabla \Psi_{m_{1}} \cdot \nabla \Psi_{m_{2}}\right) \cdots \mathscr{E}_{m_{2 q-1}} \mathscr{E}_{m_{2 q}}\left(\nabla \Psi_{m_{2 q-1}} \cdot \nabla \Psi_{m_{2 q}}\right)}{\left(\Lambda_{m_{1}}+\mathrm{i} b k\right)\left(\Lambda_{m_{2}}+\mathrm{i} b k\right) \cdots\left(\Lambda_{m_{2 q-1}}+\mathrm{i} b k\right)\left(\Lambda_{m_{2 q}}+\mathrm{i} b k\right)}\right\rangle,
\end{aligned}
$$

where $\langle\cdots\rangle$ denotes as above the ensemble average, which is equivalent to the volume average and the sums are over all eigenstates of $\mathrm{KH} \hat{H}^{\prime}$. As a next step, we average Eq. (2.28) over spatial positions of eigenstates $\Psi_{n}(\boldsymbol{r}) \equiv \Psi\left(\boldsymbol{r}-\boldsymbol{r}_{n}\right)$ as done in transition from Eq. (2.13) to Eq. (2.15). This results in the following estimate:

$$
M_{2 p, 2 q} \sim \sum_{\Lambda} \frac{\sum_{\left|\Lambda_{n}-\Lambda\right| \leq \Delta \Lambda}\left|\mathscr{E}_{n}\right|^{2 p} \mathscr{E}_{n}^{2 q}\left\langle\left(\nabla \Psi_{n} \cdot \nabla \Psi_{n}^{*}\right)^{p}\left(\nabla \Psi_{n} \cdot \nabla \Psi_{n}\right)^{q}\right\rangle}{\left(\Lambda^{2}+(b k)^{2}\right)^{p}(\Lambda+\mathrm{i} b k)^{2 q}}
$$

where the summation in the numerator is over eigenfunctions $\Psi_{n}=\Psi\left(\Lambda, \boldsymbol{r}-\boldsymbol{r}_{n}\right)$ with eigenvalues within the interval $\left|\Lambda_{n}-\Lambda\right| \leq \Delta \Lambda \ll \kappa$, while the external sum is over positions $\Lambda$ of the intervals that cover the whole range of eigenvalues $\Lambda_{n}$. The average in the numerator of Eq. (2.29) can be 

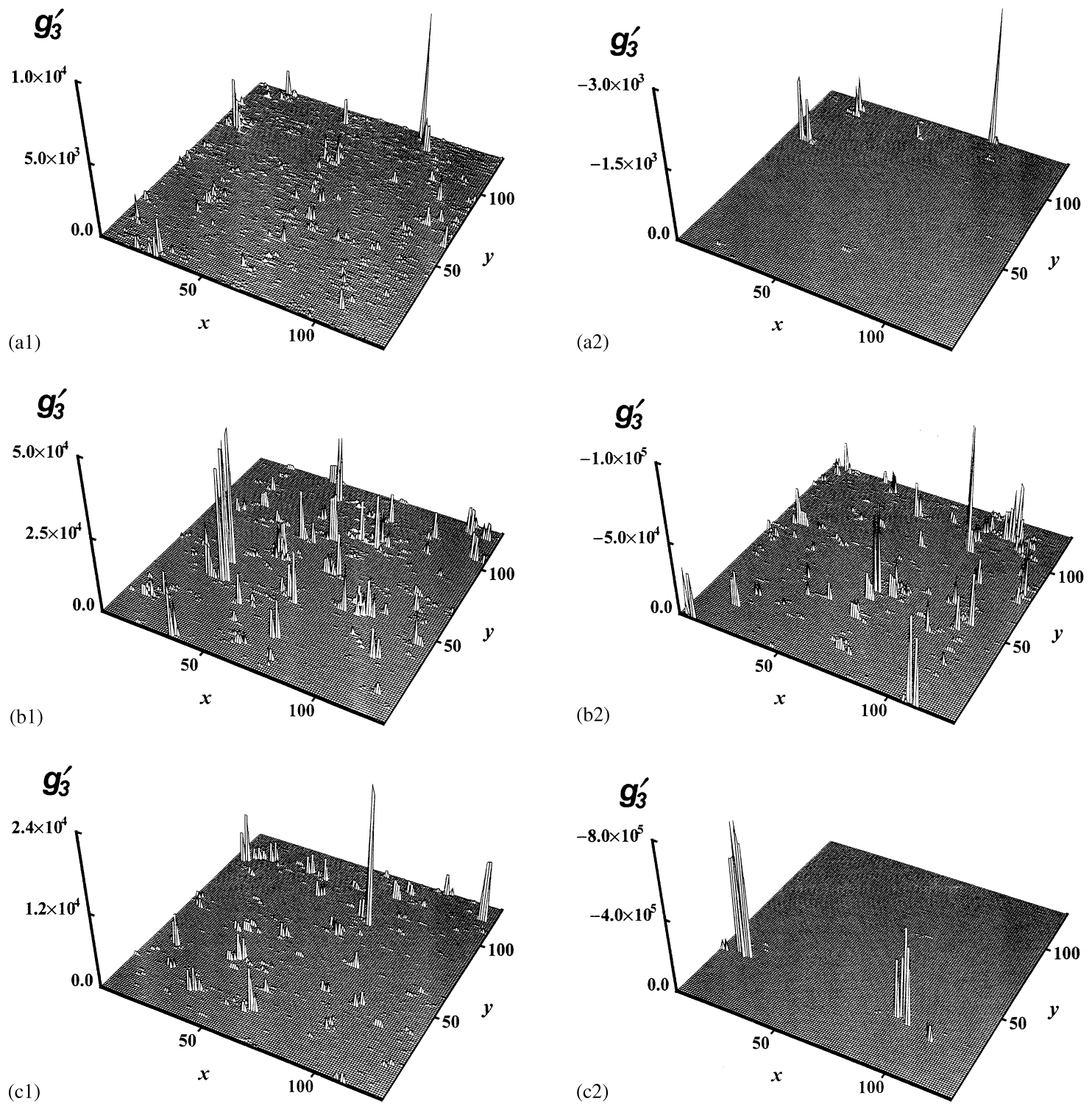

Fig. 3. Distribution of the $x$ component of the local "third harmonic field" (real part) $g_{3}^{\prime}=\operatorname{Re}\left[E^{2}(\boldsymbol{r}) E_{x}(\boldsymbol{r})\right]$ in semicontinuous silver films at wavelength $\lambda=1.5 \mu \mathrm{m}$, for different metal concentration $p$. (a1 and a2): $p=0.3$; (b1 and b2): $p=p_{c}=0.5$; (c1 and $\left.\mathrm{c} 2\right): p=0.7$. The positive $(\mathrm{a} 1, \mathrm{~b} 1, \mathrm{c} 1)$ and negative $(\mathrm{a} 2, \mathrm{~b} 2, \mathrm{c} 2)$ values of the local nonlinear fields are shown in different figures. The applied field $E_{0}=1$.

estimated as follows [see derivation of Eq. (2.19)]:

$$
\begin{aligned}
\left\langle\left(\nabla \Psi_{n} \cdot \nabla \Psi_{n}^{*}\right)^{p}\left(\nabla \Psi_{n} \cdot \nabla \Psi_{n}\right)^{q}\right\rangle & \sim \frac{1}{N \xi_{\mathrm{A}}^{2(p+q)}(\Lambda)} \sum_{i=1}^{N}\left|\Psi_{n, i}\right|^{2 p} \Psi_{n, i}^{2 q} \\
& \sim \frac{1}{N \xi_{\mathrm{A}}^{2(p+q)}(\Lambda)}\left[\frac{a}{\xi_{\mathrm{A}}}\right]^{d(p+q-1)}
\end{aligned}
$$



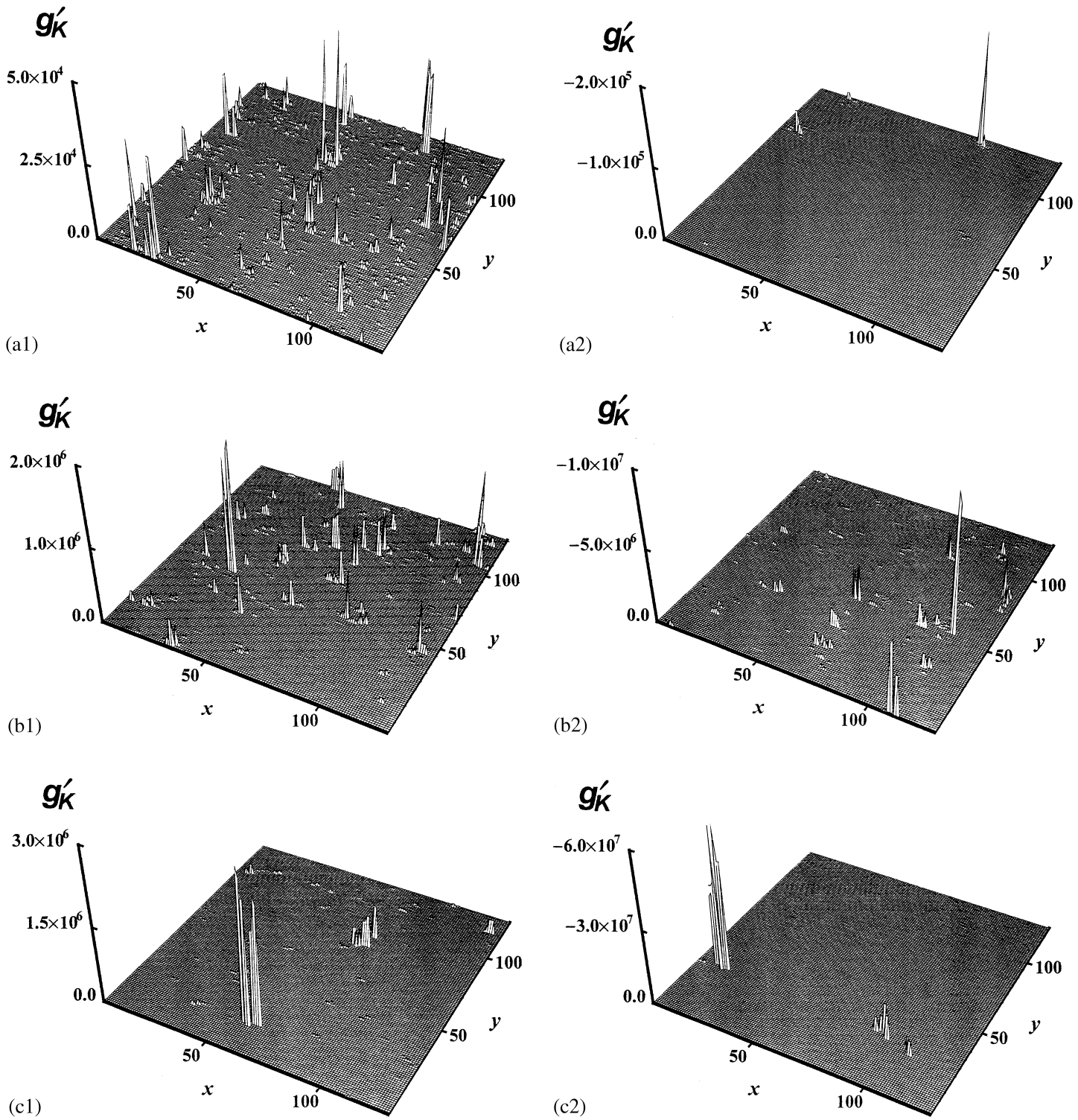

Fig. 4. Distribution of the local "Kerr field" (real part) $g_{\mathrm{K}}^{\prime}=\operatorname{Re}\left[E^{2}(\boldsymbol{r})|E(\boldsymbol{r})|^{2}\right]$ in semicontinuous silver films at wavelength $\lambda=1.5 \mu \mathrm{m}$, for different metal concentration $p$. (a1 and $\mathrm{a} 2): p=0.3$; ( $\mathrm{b} 1$ and $\mathrm{b} 2): p=p_{c}=0.5$; $(\mathrm{c} 1$ and $\mathrm{c} 2): p=0.7$. The positive $(\mathrm{a} 1, \mathrm{~b} 1, \mathrm{c} 1)$ and negative $(\mathrm{a} 2, \mathrm{~b} 2, \mathrm{c} 2)$ values of the local fields are shown in different figures. The applied field $E_{0}=1$.

where, as above, $\xi_{\mathrm{A}}(\Lambda)$ is the localization length, $a$ is the period of the square lattice in the tight binding model [see discussion after Eq. (2.5)], and $N$ is the total number of cites in the lattice. We substitute this equation and expression for $\mathscr{E}_{n}$ given by Eq. (2.18) in Eq. (2.29). Then the sum in the numerator of Eq. (2.29) takes the following form

$$
\sum_{\left|\Lambda_{n}-\Lambda\right| \leq \Delta \Lambda}\left|\mathscr{E}_{n}\right|^{2 p} \mathscr{E}_{n}^{2 q}\left\langle\left(\nabla \Psi_{n} \cdot \nabla \Psi_{n}^{*}\right)^{p}\left(\nabla \Psi_{n} \cdot \nabla \Psi_{n}\right)^{q}\right\rangle \sim \rho(\Lambda)\left[a / \xi_{\mathrm{A}}(\Lambda)\right]^{4(p+q)-d} \Delta \Lambda,
$$


where $\rho(\Lambda)$ is the dimensionless density of states [see Eq. (2.12)]. By replacing the first sum in Eq. (2.29) by integration over the spectrum we obtain

$$
M_{2 p, 2 q} \sim \int \frac{\rho(\Lambda)\left[a / \xi_{\mathrm{A}}(\Lambda)\right]^{4(p+q)-d}}{\left[\Lambda^{2}+(b \kappa)^{2}\right]^{p}(\Lambda+\mathrm{i} b \kappa)^{2 q}} \mathrm{~d} \Lambda .
$$

Note that to obtain the above expression we neglected all cross-terms in the product of eigenstates, when averaging Eq. (2.28) over the spatial positions of the eigenfunctions $\Psi_{n}=\Psi\left(\Lambda, r-r_{n}\right)$. It can be shown that after integrating over $\Lambda$, these cross-terms result in negligible [in comparison with the leading term given by Eq. (2.32)] contribution to $M_{n, m}$, for $\kappa \rightarrow 0$.

Assuming that the density of states $\rho(\Lambda)$ and the localization length $\xi_{\mathrm{A}}(\Lambda)$ are both smooth functions of $\Lambda$ in the vicinity of zero and taking into account that all parameters of the $\mathrm{KH} \hat{H}^{\prime}$ for the case $\varepsilon_{\mathrm{d}}=-\varepsilon_{\mathrm{m}}^{\prime}=1$ are of the order of one, we obtain the following estimate for the local field moments:

$$
M_{n, m}^{\star} \sim \rho(p)\left(a / \xi_{\mathrm{A}}(p)\right)^{2(n+m)-d_{1}} \kappa^{-n-m+1},
$$

for $n+m>1$ and $m>0$, where we set for simplicity $b=1$. Note that the same estimate can be obtained by considering the local fields as a set of peaks (stretched over the distance $\xi_{\mathrm{A}}$ ), with the magnitude $E_{\mathrm{m}}^{\star}$ and the average distance $\xi_{\mathrm{e}}^{\star}$ between the peaks given by Eqs. (2.23) and (2.24). Recall that the superscript ${ }^{\star}$ denotes physical quantities defined in the system with $\varepsilon_{\mathrm{d}}=-\varepsilon_{\mathrm{m}}^{\prime}=1$. In Eq. (2.23) we indicated explicitly the dependence of the density of states $\rho(p)$ and localization length $\xi_{\mathrm{A}}(p)$ on the metal concentration $p$ (as mentioned above $\rho(p)$ and $\xi_{\mathrm{A}}(p)$ are always given at $\varepsilon_{\mathrm{d}}=-\varepsilon_{\mathrm{m}}^{\prime}=1$ and the sign ${ }^{\star}$ for them is omitted). The notations $\rho(p)$ and $\xi_{\mathrm{A}}(p)$ should be understood as $\rho(p)=\rho(p, \Lambda=0)$ and $\xi_{\mathrm{A}}(p)=\xi_{\mathrm{A}}(p, \Lambda=0)$, i.e., they are given at the eigenvalue $\Lambda=0$.

The Anderson localization length $\xi_{\mathrm{A}}(\Lambda)$ has typically its maximum at the center of the distribution of the eigenvalues $\Lambda$ [74]. When $p$ departs from $1 / 2$, the value $\Lambda=0$ moves from the center of the $\Lambda$-distribution toward its wings, where the localization is typically stronger (i.e., $\xi_{\mathrm{A}}$ is less). Therefore, it is plausible to suggest that $\xi_{\mathrm{A}}(p)$ reaches its maximum at $p=1 / 2$ and decreases toward $p=0$ and $p=1$, so that the absolute value of the local field moments may have a minimum at $p=1 / 2$, according to Eq. (2.23). In $2 \mathrm{D}$ composites the percolation threshold $p_{c}$ is typically close to $p_{c} \simeq 0.5$. Therefore, the moments $M_{n, m}$ in 2D composites have a local minimum at the percolation threshold as a function of the metal concentration $p$. In accordance with this, the amplitudes of various nonlinear processes, while much enhanced, have a characteristic minimum at the percolation threshold. This localization minimum becomes more and more profound for higher optical processes.

It is important to note that the moment magnitudes in Eq. (2.33) do not depend on the number of "subtracted" (annihilated) photons in one elementary act of the nonlinear scattering. If there is at least one such photon, then the poles in Eq. (2.32) are in different complex semi-planes and the result of the integration is estimated by Eq. (2.33).

However, for the case when all photons are added (in other words, all frequencies enter the nonlinear susceptibility with the sign plus), i.e., when $n=0$, we cannot estimate the moments $M_{0, m} \equiv E_{0}^{-m} V^{-1} \int E^{m}(\boldsymbol{r}) \mathrm{d} \boldsymbol{r}$ by Eq. (2.33) since the integral in Eq. (2.32) is not further determined by the poles at $\Lambda= \pm \mathrm{i} b \kappa$. Yet all the functions of the integrand are about unity and the moment $M_{0, m}$ must be of the order of unity $M_{0, m} \sim \mathrm{O}(1)$ for $m>1$. Note that the moment $M_{0, m}$ describes, 
in particular, enhancement $G_{n \mathrm{HG}}$ of $n$-order harmonic generation, through the relation $G_{n \mathrm{HG}}=\left|M_{0, m}\right|^{2}$ (see below).

\subsection{Field fluctuations for the frequencies much below the resonance $\left(\left|\varepsilon_{\mathrm{m}}\right| \gg 1\right)$}

Above we assumed that $\left|\varepsilon_{\mathrm{m}}\right| / \varepsilon_{\mathrm{d}} \approx 1$ correspond to the plasmon resonance in the metal grains. To estimate the local field fluctuations in percolation composites for the large contrast, $\left|\varepsilon_{\mathrm{m}}\right| / \varepsilon_{\mathrm{d}} \gg 1$, we follow the scaling approach developed in Refs. $[15,38,48]$. Here we recapitulate briefly the main points of the scaling renormalization. Consider first a percolation composite where the metal concentration $p$ is equal to the percolation threshold, $p=p_{c}$. We divide a system into cubes of size $l$ and consider each cube as a new renormalized element. All such cubes can be classified into two types. A cube that contains a continuous path of metallic particles is considered as a "conducting" element. A cube without such an "infinite" cluster is considered as a nonconducting, "dielectric", element [83]. The effective dielectric constant of the "conducting" cube $\varepsilon_{\mathrm{m}}(l)$ decreases with increasing its size $l$ as $\varepsilon_{\mathrm{m}}(l) \simeq(l / a)^{-t / v} \varepsilon_{\mathrm{m}}$, whereas the effective dielectric constant of the "dielectric" cube $\varepsilon_{\mathrm{d}}(l)$ increases with $l$ as $\varepsilon_{\mathrm{d}}(l) \simeq(l / a)^{s / v} \varepsilon_{\mathrm{d}}(t, s$ and $v$ are the percolation critical exponents for the static conductivity, dielectric constant, and percolation correlation length, respectively; for $2 \mathrm{D}$ case, $t \cong s \cong v \cong 4 / 3$, in $3 \mathrm{D}$, the exponents are equal to $t \simeq 2.0, s \simeq 0.7$, and $v \simeq 0.88[12,64]$ ). We set now the cube size $l$ to be equal to

$$
l=l_{\mathrm{r}}=a\left(\left|\varepsilon_{\mathrm{m}}\right| / \varepsilon_{\mathrm{d}}\right)^{v /(t+s)} .
$$

Then, in the renormalized system, where each cube of size $l_{\mathrm{r}}$ is considered as a single element, the dielectric constant of these new elements takes either value $\varepsilon_{\mathrm{m}}\left(l_{\mathrm{r}}\right)=\varepsilon_{\mathrm{d}}^{t /(t+s)}\left|\varepsilon_{\mathrm{m}}\right|{ }^{s /(t+s)}\left(\varepsilon_{\mathrm{m}} /\left|\varepsilon_{\mathrm{m}}\right|\right)$, for the element renormalized from the conducting cube, or $\varepsilon_{\mathrm{d}}\left(l_{\mathrm{r}}\right)=\varepsilon_{\mathrm{d}}^{t(t+s)}\left|\varepsilon_{\mathrm{m}}\right|^{s /(t+s)}$, for the element renormalized from the dielectric cube. The ratio of the dielectric constants of these new elements is equal to $\varepsilon_{\mathrm{m}}\left(l_{\mathrm{r}}\right) / \varepsilon_{\mathrm{d}}\left(l_{\mathrm{r}}\right)=\varepsilon_{\mathrm{m}} /\left|\varepsilon_{\mathrm{m}}\right| \cong-1+\mathrm{i} \kappa$, where the loss-factor $\kappa=\varepsilon_{\mathrm{m}}^{\prime \prime} /\left|\varepsilon_{\mathrm{m}}\right| \ll 1$ is the same as in the original system. According to the basic ideas of the renormalization group transformation [64,83], the concentration of conducting and dielectric elements does not change under the above transformation, provided that $p=p_{c}$. The field distribution in a two component system depends on the ratio of the dielectric permittivities of the components. Thus after the renormalization, the problem becomes equivalent to the above considered field distribution for the case $\varepsilon_{\mathrm{d}}=-\varepsilon_{\mathrm{m}}^{\prime}=1$. Taking into account that the electric field renormalizes as $E_{0}^{\star}=E_{0}\left(l_{\mathrm{r}} / a\right)$, we obtain from Eq. (2.23) that the field peaks in the renormalized system are

$$
E_{\mathrm{m}} \simeq E_{0}\left(a / \xi_{\mathrm{A}}\right)^{2}\left(l_{\mathrm{r}} / a\right) \kappa^{-1} \simeq E_{0}\left(a / \xi_{\mathrm{A}}\right)^{2}\left(\frac{\left|\varepsilon_{\mathrm{m}}\right|}{\varepsilon_{\mathrm{d}}}\right)^{v /(t+s)}\left(\frac{\left|\varepsilon_{\mathrm{m}}\right|}{\varepsilon_{\mathrm{m}}^{\prime \prime}}\right),
$$

where $\xi_{\mathrm{A}}=\xi_{\mathrm{A}}\left(p_{c}\right)$ is the localization length in the renormalized system. In the original system, each field maximum of the renormalized system locates in a dielectric gap in the "dielectric" cube of the $l_{\mathrm{r}}$ size or in-between two "conducting" cubes of the size $l_{\mathrm{r}}$ that are not necessarily connected to each other [83]. There is no characteristic length in the original system which is smaller than $l_{\mathrm{r}}$, except the microscopical length in the problem, which is grain size $a$. Therefore, it is plausible to suggest that the width of a local field peak in the original system is about $a$. Then the values of the field maxima $E_{\mathrm{m}}$ do not change when returning from the renormalized system to the original one. Therefore, Eq. (2.35) gives the values of the field maxima in the original system. Note that the value 
$E_{\mathrm{m}}$ of the field maxima is different from previously obtained estimate (2.23) due to the renormalization of the applied field $E_{0}$.

Eq. (2.35) gives the estimate for the local field extrema when the real part $\varepsilon_{\mathrm{m}}^{\prime}$ of the metal dielectric constant becomes negative. For metals $\varepsilon_{\mathrm{m}}$ increases in absolute value with the wavelength, when the frequency $\omega$ is smaller than $\tilde{\omega}_{\mathrm{p}}$ [see discussion below Eq. (2.1)]. Therefore, the field peaks $E_{\mathrm{m}}(\omega)$ increase strongly with the wavelength (see, for example, Fig. 2 taken from [15]). For a Drude metal the steep growth of the peaks $E_{\mathrm{m}}(\omega)$ occurs for the frequencies $\omega \lesssim \tilde{\omega}_{\mathrm{p}}$, when the dielectric constant $\varepsilon_{\mathrm{m}}$ can be approximated as

$$
\varepsilon_{\mathrm{m}}\left(\omega \lesssim \tilde{\omega}_{\mathrm{p}}\right) \cong 2\left(\omega-\tilde{\omega}_{\mathrm{p}}\right) \frac{\varepsilon_{\mathrm{b}}}{\tilde{\omega}_{\mathrm{p}}}+\mathrm{i} \frac{\varepsilon_{\mathrm{b}} \omega_{\tau}}{\tilde{\omega}_{\mathrm{p}}} .
$$

By substituting this expansion in Eq. (2.35), we obtain

$$
E_{\mathrm{m}}\left(\omega \lesssim \tilde{\omega}_{\mathrm{p}}\right) \simeq E_{0}\left(a / \xi_{\mathrm{A}}\right)^{2}\left(\frac{2 \varepsilon_{\mathrm{b}}\left|\omega-\tilde{\omega}_{\mathrm{p}}\right|}{\tilde{\omega}_{\mathrm{p}}}\right)^{(v+t+s) /(t+s)} \frac{\tilde{\omega}_{\mathrm{p}}}{\omega_{\tau} \varepsilon_{\mathrm{b}} \varepsilon_{\mathrm{d}}^{v /(t+s)}} .
$$

Since losses in a typical metal are small, $\omega_{\tau} \ll \tilde{\omega}_{\mathrm{p}}$, the field peak amplitudes first increase steeply and then saturate (see below) at the magnitude $E_{\mathrm{m}} \simeq E_{0}\left(a / \xi_{\mathrm{A}}\right)^{2}\left(\varepsilon_{\mathrm{b}} / \varepsilon_{\mathrm{d}}\right)^{v /(t+s)}\left(\tilde{\omega}_{\mathrm{p}} / \omega_{\tau}\right) \sim E_{0} \tilde{\omega}_{\mathrm{p}} / \omega_{\tau}$ when $\omega \approx 0.5 \tilde{\omega}_{\mathrm{p}}$. Therefore, the intensity maxima $I_{\mathrm{m}}$ exceed the intensity of the incident wave $I_{0}$ by the factor $I_{\mathrm{m}} / I_{0} \sim\left(\tilde{\omega}_{\mathrm{p}} / \omega_{\tau}\right)^{2} \gg 1$. For a silver-glass percolation composite we obtained $I_{\mathrm{m}} / I_{0} \sim 10^{3}$ (see also the field distribution in Figs. 1 and 2).

Now we consider the case of small frequencies $\omega \ll \omega_{\mathrm{p}}$ when the dielectric constant $\varepsilon_{\mathrm{m}}$ for a Drude metal [see Eq. (2.1)] takes the form

$$
\varepsilon_{\mathrm{m}}\left(\omega \ll \omega_{\mathrm{p}}\right) \cong-\left(\frac{\omega}{\omega_{\mathrm{p}}}\right)^{2}\left(1-\mathrm{i} \frac{\omega_{\tau}}{\omega}\right),
$$

where we again assume that $\omega \gg \omega_{\tau}$. By substituting this expression in Eq. (2.35), we obtain

$$
E_{\mathrm{m}}\left(\omega \ll \omega_{\mathrm{p}}\right) \simeq E_{0}\left(\frac{a}{\xi_{\mathrm{A}}}\right)^{2}\left(\frac{\omega_{\mathrm{p}}}{\sqrt{\varepsilon_{\mathrm{d}}} \omega}\right)^{2 v /(t+s)}\left(\frac{\omega}{\omega_{\tau}}\right) .
$$

For the $2 \mathrm{D}$ case, the critical exponents are equal to $v \cong t \cong s \cong 4 / 3$ and Eq. (2.39) gives $E_{\mathrm{m}} \sim E_{0}\left(a / \xi_{\mathrm{A}}\right)^{2} \omega_{\mathrm{p}} /\left(\sqrt{\varepsilon_{\mathrm{d}}} \omega_{\tau}\right)=E_{0}\left(a / \xi_{\mathrm{A}}\right)^{2}\left(\tilde{\omega}_{\mathrm{p}} / \omega_{\tau}\right) \sqrt{\varepsilon_{\mathrm{b}} / \varepsilon_{\mathrm{d}}} \sim E_{0}\left(\tilde{\omega}_{\mathrm{p}} / \omega\right)$ and that coincides with the estimate obtained from Eq. (2.37) for $\omega=0.5 \tilde{\omega}_{\mathrm{p}}$. This means that the local field peaks increase steeply when the real part of the metal dielectric constant $\varepsilon_{\mathrm{m}}$ becomes negative $\varepsilon_{\mathrm{m}}^{\prime}<0$ and then remains almost the same in the wide frequency range $\tilde{\omega}_{\mathrm{p}}>\omega>\omega_{\tau}$, for $2 \mathrm{D}$ composites.

For $3 \mathrm{D}$ percolation composites, the critical exponents are equal to $v \cong 0.88, t \cong 2.0, s \cong 0.7$ [12]. To simplify estimations we put below $v \cong(t+s) / 3$ for $d=3$. Then Eq. (2.39) takes the following form $E_{\mathrm{m}} \sim E_{0}\left(\varepsilon_{\mathrm{b}} / \varepsilon_{\mathrm{d}}\right)^{1 / 3} \tilde{\omega}_{\mathrm{p}}^{2 / 3} \omega^{1 / 3} / \omega_{\tau}$, that is the local field peaks increase up to $E_{\mathrm{m}} / E_{0} \sim \tilde{\omega}_{\mathrm{p}} / \omega_{\tau}$ when $\varepsilon_{\mathrm{m}}^{\prime}$ becomes negative and then the peaks decrease as $E_{\mathrm{m}} / E_{0} \sim\left(\tilde{\omega}_{\mathrm{p}} / \omega_{\tau}\right)\left(\omega / \tilde{\omega}_{\mathrm{p}}\right)^{1 / 3}$, with further decrease of frequency. For silver composites, we estimate that the maximum value of the peaks is achieved at $\omega \cong 0.5 \tilde{\omega}_{\mathrm{p}}$ that corresponds to $\lambda \cong 0.6 \mu \mathrm{m}$.

Since we know the peak amplitudes for the local electric field we can estimate the moments $M_{n, m}$ of the local field. To obtain $M_{n, m}$ we consider first the spatial distribution of the field maxima for $\left|\varepsilon_{\mathrm{m}}\right| \gg \varepsilon_{\mathrm{d}}$. The average distance between the field maxima in the renormalized system is equal to 
$\xi_{\mathrm{e}}^{\star}$ given by Eq. (2.24). Then the average distance $\xi_{\mathrm{e}}$ between the field maxima in the original system (provided that $\rho \sim 1$ ) is equal to

$$
\xi_{\mathrm{e}} \cong\left(l_{\mathrm{r}} / a\right) \xi_{\mathrm{e}}^{\star} \sim a\left(\frac{\left|\varepsilon_{\mathrm{m}}\right|}{\varepsilon_{\mathrm{d}}}\right)^{v /(t+s)}\left(\frac{\left|\varepsilon_{\mathrm{m}}\right|}{\varepsilon_{\mathrm{m}}^{\prime \prime}}\right)^{1 / d} .
$$

Note that in the $2 \mathrm{D}$ case this ascribes a simple form

$$
\xi_{\mathrm{e}} \sim a \frac{\left|\varepsilon_{\mathrm{m}}\right|}{\sqrt{\varepsilon_{\mathrm{d}} \ni \varphi \omega \#_{\mathrm{m}}^{\mathrm{e}}}},
$$

where the critical exponents for $2 \mathrm{D}$ percolating composites, $t \cong s \cong v \cong 4 / 3$ are used. In the renormalized system a typical "area" of a field peak corresponds to $d$ power of the Anderson localization length $\xi_{\mathrm{A}}$. Therefore, in the original system each field maximum is stretched over $\left(\xi_{\mathrm{A}} / a\right)^{d}$ clusters of size $l_{\mathrm{r}}$. In each of these clusters the field maximum splits into $n\left(l_{\mathrm{r}}\right)$ peaks of the $E_{\mathrm{m}}$ amplitude located along a dielectric gap in the "dielectric" square of the $l_{\mathrm{r}}$ size. The gap "area" scales as the capacitance of the dielectric squares, so does the number of peaks

$$
n\left(l_{\mathrm{r}}\right) \propto\left(l_{\mathrm{r}} / a\right)^{d-2+s / v} .
$$

Multiplying the amplitude of the field peaks $E_{\mathrm{m}}$ raised to the proper power by the number of the peaks in one group $\left(\xi_{\mathrm{A}} / a\right)^{d} n\left(l_{\mathrm{r}}\right)$ and normalizing to the distance between the groups $\xi_{\mathrm{e}}$ we obtain the following estimate for the local-field moments:

$$
\begin{aligned}
M_{n, m} & \sim\left(\xi_{\mathrm{A}} / a\right)^{d}\left(\frac{E_{\mathrm{m}}}{E_{0}}\right)^{n+m} \frac{n\left(l_{\mathrm{r}}\right)}{\left(\xi_{\mathrm{e}} / a\right)^{d}} \sim \rho\left(\xi_{\mathrm{A}} / a\right)^{d-2(n+m)}\left(l_{\mathrm{r}} / a\right)^{n+m+s / v-2} \kappa^{1-n-m} \\
& \sim \rho\left(\xi_{\mathrm{A}} / a\right)^{d-2(n+m)}\left(\frac{\left|\varepsilon_{\mathrm{m}}\right|}{\varepsilon_{\mathrm{d}}}\right)^{[(n+m-2) v+s] /(t+s)}\left(\frac{\left|\varepsilon_{\mathrm{m}}\right|}{\varepsilon_{\mathrm{m}}^{\prime \prime}}\right)^{n+m-1}
\end{aligned}
$$

that holds for $n+m>1$ and $n>0$. Since $\left|\varepsilon_{\mathrm{m}}\right| \gg \varepsilon_{\mathrm{d}}$ and $\left|\varepsilon_{\mathrm{m}}\right| / \varepsilon_{\mathrm{m}}^{\prime \prime} \gg 1$ the moments of the local field are very large, $M_{n, m} \gg 1$, in the visible and infrared spectral ranges. Note that the first moment $M_{0,1} \sim 1$ that corresponds to the equation $\langle\boldsymbol{E}(\boldsymbol{r})\rangle=\boldsymbol{E}_{0}$. We stress again that the localization length $\xi_{\mathrm{A}}$ in Eq. (2.43) corresponds to the renormalized system with $\varepsilon_{\mathrm{d}}=-\varepsilon_{\mathrm{m}}^{\prime}=1$. The localization length in the original system, i.e., a typical size of the eigenfunction is estimated as $\left(l_{\mathrm{r}} / a\right) \xi_{\mathrm{A}} \gg a$. In other words, the eigenstates become macroscopically large in the limit of large contrast $\left|\varepsilon_{\mathrm{m}}\right| / \varepsilon_{\mathrm{d}} \gg 1$ and consist of sharp peaks separated in space by distances much larger than $a$. The eigenstates of HK $\hat{H}$ cover the volume $\left(\xi_{\mathrm{A}} l_{\mathrm{r}} / a\right)^{d} \sim\left(\xi_{\mathrm{A}} \tilde{\omega}_{\mathrm{p}} / \omega\right)^{d} \gg a^{d}$ for two-dimensional Drude metal composites and $\omega \ll \omega_{\mathrm{p}}$.

We consider now the moments $M_{n, m}$ for $n=0$ that correspond to the volume average of the $m$ th power of the complex amplitude $E(\boldsymbol{r})$, namely, $M_{0, m}=\left\langle E^{m}(\boldsymbol{r})\right\rangle /\left|E_{0}\right|^{m}$. In the renormalized system, where $\left|\varepsilon_{\mathrm{m}}\left(l_{\mathrm{r}}\right)\right|=\left|\varepsilon_{\mathrm{d}}\left(l_{\mathrm{r}}\right)\right|$ and $\varepsilon_{\mathrm{m}}\left(l_{\mathrm{r}}\right) / \varepsilon_{\mathrm{d}}\left(l_{\mathrm{r}}\right) \cong-1+\mathrm{i} \kappa$, the field distribution coincides with the field distribution in the system with $\varepsilon_{\mathrm{d}} \simeq-\varepsilon_{\mathrm{m}}^{\prime} \sim 1$. In the system with $\varepsilon_{\mathrm{d}} \simeq-\varepsilon_{\mathrm{m}}^{\prime} \sim 1$ the field peaks $E_{\mathrm{m}}^{\star}$ are different in phase and because of the destructive interference, the moment $M_{0, m}^{\star} \sim \mathrm{O}(1)$ [as follows from Eq. (2.32)]. In transition to the original system the peaks increase by the factor $l_{\mathrm{r}} / a$, leading to the corresponding increase of the moment $M_{0, m}$. We suppose that within a single "dielectric" cube the field peaks are in phase, i.e., the field maxima form chains of aligned peaks which are stretched out in a dielectric cube. This assumption is confirmed by results of numerical 
simulation shown in Fig. 3, where the field maxima with different signs are concentrated in different places of a percolation composite. Then we obtain the following equation for the moment:

$$
M_{0, m} \sim M_{0, m}^{\star}\left(l_{\mathrm{r}} / a\right)^{m} \frac{n\left(l_{\mathrm{r}}\right)}{\left(\xi_{\mathrm{e}} / a\right)^{d}} \sim \kappa\left(l_{\mathrm{r}} / a\right)^{m-2+s / v} \sim\left(\frac{\varepsilon_{\mathrm{m}}^{\prime \prime}}{\left|\varepsilon_{\mathrm{m}}\right|}\right)\left(\frac{\left|\varepsilon_{\mathrm{m}}\right|}{\varepsilon_{\mathrm{d}}}\right)^{(m-2+s / v) v /(t+s)},
$$

which holds when $M_{0, m}$ given by this equation is larger than one.

Using the critical exponents for $2 \mathrm{D}$ percolating composites, $t \cong s \cong v \cong 4 / 3$ [12], we can simplify Eqs. (2.43) and (2.44) as follows:

$$
M_{n, m} \sim \rho\left(\frac{\left|\varepsilon_{\mathrm{m}}\right|^{3 / 2}}{\left(\xi_{\mathrm{A}} / a\right)^{2} \sqrt{\varepsilon_{\mathrm{d}} \varepsilon_{\mathrm{m}}^{\prime \prime}}}\right)^{n+m-1} \quad(d=2),
$$

for $n+m>1$ and $n>0$, and

$$
M_{0, m} \sim \frac{\varepsilon_{\mathrm{m}}^{\prime \prime}\left|\varepsilon_{\mathrm{m}}\right|^{(m-3) / 2}}{\varepsilon_{\mathrm{d}}^{(m-1) / 2}} \quad(d=2),
$$

for $m>1, n=0$ and $\left(\left|\varepsilon_{\mathrm{m}}\right| / \varepsilon_{\mathrm{d}}\right)^{(m-1) / 2}>\left|\varepsilon_{\mathrm{m}}\right| / \varepsilon_{\mathrm{m}}^{\prime \prime}$ (the last inequality corresponds to the condition that the moment given by Eq. (2.46) is larger than one).

The moments $M_{n, m}(n \neq 0)$ are strongly enhanced in 2D Drude metal-dielectric composites. The moments reach the maximum value

$$
M_{n, m} \sim \rho\left(\frac{\omega_{\mathrm{p}}}{\omega_{\tau} \sqrt{\varepsilon_{\mathrm{d}}}\left(\xi_{\mathrm{A}} / a\right)^{2}}\right)^{n+m-1} \quad(d=2),
$$

when frequency $\omega$ decreases so that the condition $\omega \ll \omega_{\mathrm{p}}$ is fulfilled. The spatial moments of the local electric in a 2D percolation composite are independent of frequency, for $\omega \ll \omega_{\mathrm{p}}$. For metals it typically takes place in the red and infrared spectral ranges. For a silver semicontinuous film on a glass substrate, the moment $M_{n, m}$ can be estimated as $M_{n, m} \sim\left[\left(a / \xi_{\mathrm{A}}\right)^{2} 3 \times 10^{2}\right]^{n+m-1}$, for $\omega \ll \omega_{\mathrm{p}}$.

It follows from Eq. (2.43) that for 3D metal-dielectric percolation composites, where the dielectric constant of metal component can be estimated by the Drude formula (2.1), the moments $M_{n, m}(n \neq 0)$ achieve the maximum value at frequency $\omega_{\max } \approx 0.5 \tilde{\omega}_{\mathrm{p}}$. To estimate the maximum value, we note that the following relations $v /(t+s) \approx 1 / 3, s \approx v$ are valid for the $3 \mathrm{D}$ case, where $t \cong 2.0, s \cong 0.7$ and $v \cong 0.88$ [12]. Then the maximum value of the moments is estimated as

$$
M_{n, m}\left(\omega=\omega_{\max }\right) \sim \rho\left(\xi_{\mathrm{A}} / a\right)\left[\left(a / \xi_{\mathrm{A}}\right)^{2}\left(\varepsilon_{\mathrm{b}} / \varepsilon_{\mathrm{d}}\right)^{1 / 3} \tilde{\omega}_{\mathrm{p}} / \omega_{\tau}\right]^{n+m-1} \quad(d=3) .
$$

For small frequencies $\omega \ll \omega_{\mathrm{p}}$, the moments of the local field decrease with the wavelength as

$$
M_{n, m}\left(\omega \ll \omega_{\mathrm{p}}\right) \sim \rho\left(\xi_{\mathrm{A}} / a\right)\left[\frac{\left(a / \xi_{\mathrm{A}}\right)^{2} \omega_{\mathrm{p}}^{2 / 3} \omega^{1 / 3}}{\varepsilon_{\mathrm{d}}^{1 / 3} \omega_{\tau}}\right]^{n+m-1} \quad(d=3) .
$$

In Fig. 5 we compare results of numerical and theoretical calculations for the field moments in 2D silver semicontinuous films on glass. We see that there is excellent agreement between the scaling theory [formulas (2.45) and (2.46)] and numerical simulations. To fit the data we used $\xi_{\mathrm{A}} \approx 2 a$. [Results of numerical simulations for $M_{0,4}$ are not shown in Fig. 5 since it was not 


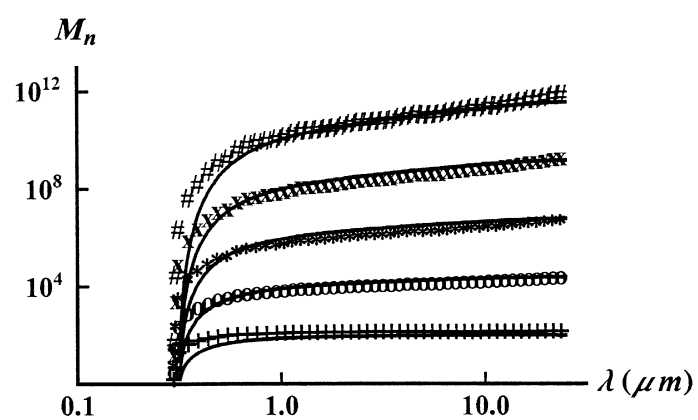

(a)

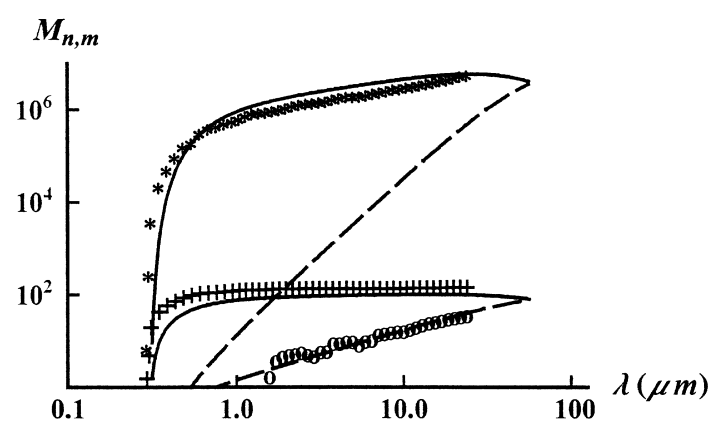

(b)

Fig. 5. High-order field moments of local electric field in semicontinuous silver films as a function of the wavelength $\lambda$ at $p=p_{c}$. (a) Results of numerical calculations of the moments $M_{n} \equiv M_{n, 0}=\left\langle\left|\boldsymbol{E}_{1}(\boldsymbol{r})\right|^{n}\right\rangle /\left|\left\langle\boldsymbol{E}_{1}\right\rangle\right|^{n}$ for $n=2,3,4,5$ and 6 are represented by $+, \bigcirc, *, \times$, and \#, respectively. The solid lines describe $M_{n}$ found from the scaling formula (2.43). (b) Comparison of the moment $M_{4,0}=\left\langle\left|\boldsymbol{E}_{1}(\boldsymbol{r})\right|^{4}\right\rangle /\left|\left\langle\boldsymbol{E}_{1}\right\rangle\right|^{4}$ [upper solid line - scaling formula (2.43), * numerical simulations] and moment $M_{0,4}=\left|\left\langle\boldsymbol{E}_{1}^{4}(\boldsymbol{r})\right\rangle\right| /\left|\left\langle\boldsymbol{E}_{1}\right\rangle\right|^{4}$ [upper dashed line - scaling formula (2.44)]. The moment $M_{2,0}=\left\langle\left|\boldsymbol{E}_{1}(\boldsymbol{r})\right|^{2}\right\rangle /\left|\left\langle\boldsymbol{E}_{1}\right\rangle\right|^{2}$ [lower solid line - scaling formula (2.43), + - numerical simulations] vs. moment $M_{0,2}=\left|\left\langle\boldsymbol{E}_{1}(\boldsymbol{r})^{2}\right\rangle\right| /\left|\left\langle\boldsymbol{E}_{1}\right\rangle\right|^{2}$ [lower dashed line - scaling formula (2.44), $\bigcirc$ - numerical simulations]. In all presented analytical calculations we set $\xi_{\mathrm{A}}=2 a$ and $\rho=1$ in Eqs. (2.43) and (2.44).

possible to achieve reliable results in the simulations because of large fluctuations in values of this moment.] A small value of $\xi_{\mathrm{A}}$ indicates strong localization of surface plasmons in percolation composites, at least for the 2D case. As seen in Fig. 5b the spectral dependence of enhancement $M_{n, m}$ differs strongly for processes with $(n \neq 0)$ and without $(n=0)$ subtraction of photons.

As discussed in the introduction, nonlinear optical processes, in general, are phase dependent and proportional to a factor $|E|^{n} E^{m}$, i.e., they depend on the phase through the term $E^{m}$ and their enhancement is estimated as $M_{n, m}=\left\langle\left|E / E_{0}\right|^{n}\left(E / E_{0}\right)^{m}\right\rangle$. According to the above consideration, $M_{n, m} \sim M_{n+m, 0} \equiv M_{n+m}$, for $n \geq 1$. For example, enhancement of the Kerr-type nonlinearity $G_{\mathrm{K}}=M_{2,2}$ is similar to the enhancement of the Raman scattering $G_{\mathrm{RS}} \simeq M_{4}$ (see Sections 5 and 6). For nearly degenerate four-wave mixing (FWM), the enhancement is given by $G_{\mathrm{FWM}} \sim\left|G_{\mathrm{K}}\right|^{2} \sim\left|M_{2,2}\right|^{2}$ and can reach giant values up to $\sim 10^{12}$.

Above, for the sake of simplicity, we assumed that $p=p_{c}$ when considering the case of $\varepsilon_{\mathrm{m}}^{\prime} \ll 0$. Now we estimate the concentration range $\Delta p=p-p_{c}$, where the above estimates for the local field moments are valid [37,38]. We note that the above expressions for the local field and average field moments $M_{n, m}$ hold in almost all concentration range given by Eq. (2.25) when $\varepsilon_{\mathrm{m}} \simeq-\varepsilon_{\mathrm{d}}$. The metal concentration range $\Delta p$, where the local electric field is strongly enhanced, shrinks, however, when $\varepsilon_{\mathrm{m}}^{\prime} \ll 0$. The above speculations are based on the finite size scaling arguments, which hold provided the scale $l_{\mathrm{r}}$ of the renormalized cubes is smaller than the percolation correlation length $\xi_{\mathrm{p}} \cong a\left(\left|p-p_{c}\right| / p_{c}\right)^{-v}$. At the percolation threshold, where the correlation length $\xi_{\mathrm{p}}$ diverges, our estimates are valid in the wide frequency range $\omega_{\tau}<\omega<\tilde{\omega}_{\mathrm{p}}$ which includes the visible, infrared, and far-infrared spectral ranges for a typical metal. For any particular frequency from this interval, we estimate the concentration range $\Delta p$ (where the giant field fluctuations occur) by equating the values of $l_{\mathrm{r}}$ and $\xi_{\mathrm{p}}$, which results in the inequality $|\Delta p| \leq\left(\varepsilon_{\mathrm{d}} /\left|\varepsilon_{\mathrm{m}}\right|\right)^{1 /(t+s)}$. Therefore, the local electric field fluctuates strongly for these concentrations and its moments $M_{n, m}$ are strongly enhanced. 


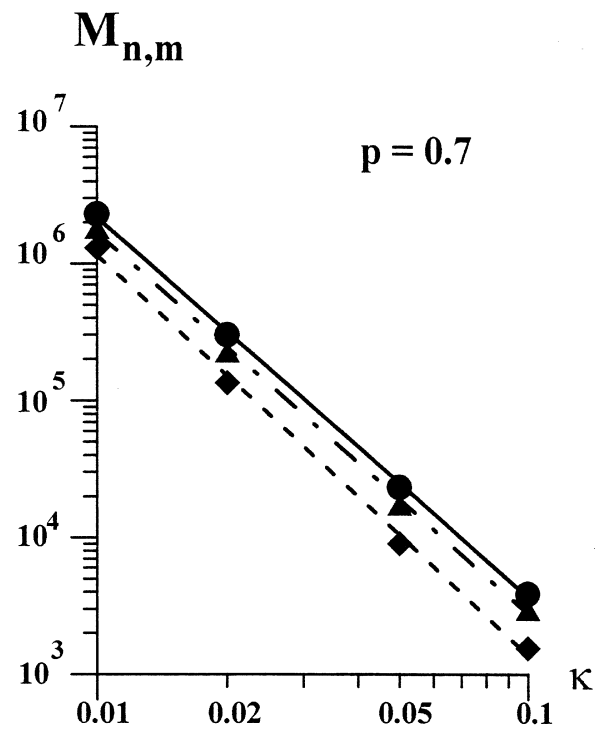

Fig. 6. Fourth-order field moments $M_{m, n}(m+n=4)$ of the local electric field in $2 d$ metal-dielectric composite with $\varepsilon_{\mathrm{d}}=1$ and metal permittivity $\varepsilon_{\mathrm{m}}=-100(1-\mathrm{i} \kappa)$, as functions of $\kappa: M_{4,0}-\boldsymbol{O}, M_{3,1}-\boldsymbol{\Delta}, M_{2,2}-\mathbf{\square}$.

In Fig. 6 we show the moments $M_{4,0}, M_{3,1}$ and $M_{2,2}$ as a function of $\kappa$ for $2 \mathrm{D}$ percolating system with $\varepsilon_{\mathrm{m}}=100(-1+\mathrm{i} \kappa), \varepsilon_{\mathrm{d}}=1$ and metal concentration $p=0.7>p_{c}=0.5$. All the moments are close in magnitude and increase with decreasing losses $\kappa$ according to a power-law dependence with the same exponent, as it is predicted by Eq. (2.45).

\section{Numerical and experimental studies of field distributions in two-dimensional percolation composites}

The optical properties of metal-insulator thin films have been intensively studied both experimentally and theoretically. Semicontinuous metal films with a two-dimensional (2D) morphology are usually produced by thermal evaporation or spattering of metal onto an insulating substrate. In the growing process, first, small metallic grains are formed on the substrate. As the film grows, the metal filling factor increases and coalescences occur, so that irregularly shaped clusters are formed on the substrate resulting in 2D fractal structures. The sizes of these structures diverge in a vicinity of the percolation threshold. A percolating cluster of metal is eventually formed, when a continuous conducting path appears between the ends of the sample. The metal-insulator transition (the percolation threshold) is very close to this point, even in the presence of quantum tunneling. At higher surface coverage, the film is mostly metallic, with voids of irregular shapes. As further coverage increase, the film becomes uniform.

The optical properties of metal-dielectric films show anomalous phenomena that are absent for bulk metal and dielectric components. For example, the anomalous absorption in the nearinfrared spectral range leads to unusual behavior of transmittance and reflectance. Typically, the 
transmittance is much higher than that of continuous metal films, whereas the reflectance is much lower (see Refs. [12,19,20,64,84-86] and references therein). Near and well-below the conductivity threshold, the anomalous absorptance can be as high as 50\% [85,88-91]. A number of the effective-medium theories were proposed for calculation of the optical properties of semicontinuous random films, including the Maxwell-Garnett [92] and Bruggeman [49] approaches and their various modifications [12,85-87]. The renormalization group method is also widely used to calculate effective dielectric response of 2D percolation films near the percolation threshold (see $[93,94]$ and references therein). However, none of these theories allows one to calculate the field fluctuations and the effects resulting from these fluctuations.

Because semicontinuous metal films are of great interest in terms of their fundamental physical properties and various applications, it is important to study statistical properties of the electromagnetic fields in the near-zone of these films. To simplify theoretical considerations, we assume below that the electric field is homogeneous in the direction perpendicular to the film plane. This assumption means that the skin depth for the metal grains, $\delta \cong c /\left(\omega \sqrt{\left|\varepsilon_{\mathrm{m}}\right|}\right)$, is much larger than the grain size, $a$, so that the quasistatic approximation holds. Note that the role of the skin effect can be very important resulting, in many cases, in strong alterations of the electromagnetic response found in the quasistatic approximation [39,95-97]. These effects will be discussed in Section 7. Yet, the quasistatic approximation significantly simplifies theoretical considerations of the field fluctuations and describes well the optical properties of semicontinuous films providing qualitative (and in some cases, quantitative) agreement with experimental data [12,20,98,99].

Below, we neglect the skin effect so that a semicontinuous film can be considered as a $2 \mathrm{D}$ object. In the optical frequency range, when the frequency, $\omega$, is much larger than the relaxation rate $\tau^{-1}$ of the metallic component, a semicontinuous metal film can be thought of as a $2 \mathrm{D} L-R-C$ lattice $[12,20,98,99]$. As above, the capacitance $C$ stands for the gaps between metal grains that are filled by dielectric material (substrate), with the dielectric constant $\varepsilon_{\mathrm{d}}$. The inductive elements, $L-R$, represent the metallic grains that for the Drude metal have the dielectric function $\varepsilon_{\mathrm{m}}(\omega)$ given by Eq. (2.1). In the high-frequency range considered here, the losses in metal grains are small, $\omega \gg \omega_{\tau}$. Therefore, the real part of the metal dielectric function is much larger (in modulus) than the imaginary part and it is negative for the frequencies $\omega$ below the renormalized plasma frequency, $\tilde{\omega}_{\mathbf{p}}=\omega_{\mathrm{p}} / \sqrt{\varepsilon_{\mathrm{b}}}$. Thus, the metal conductivity is almost purely imaginary and metal grains can be modeled as the $L-R$ elements, with the active component much smaller than the reactive one.

If the skin effect cannot be neglected, i.e., the skin depth $\delta$ is smaller than the metal grain size $a$ the simple quasistatic presentation of a semicontinuous film as a $2 \mathrm{D}$ array of the $L-R$ and $C$ elements is not valid. Still, we can use the $L-R-C$ model in the other limiting case, when the skin effect is very strong, $\delta \ll a[95,96]$. In this case, the losses in metal grains are small, regardless of the ratio $\omega / \omega_{\tau}$, whereas the effective inductance for a metal grain depends on the grain size and shape rather than on the material constants for the metal. Properties of metal-dielectric composites beyond the quasistatic approximation will be discussed in detail in Section 7.

The effective properties of the $2 \mathrm{D} L-R-C$ lattices have been intensively studied during the last decade $[12,20,98,99]$. However, there was not much attention paid to the fact that the spatial distributions of the local fields in such systems can exhibit rich nontrivial behavior.

It is instructive to consider first the film properties at the percolation threshold, $p=p_{c}$, where the exact result for the effective dielectric constant $\varepsilon_{\mathrm{e}}$ holds in the quasistatic case [100]: $\varepsilon_{\mathrm{e}}=\sqrt{\varepsilon_{\mathrm{d}} \varepsilon_{\mathrm{m}}}$. If we neglect the metal losses and put $\omega_{\tau}=0$, the metal dielectric constant $\varepsilon_{\mathrm{m}}$ is negative for 
frequencies smaller than the renormalized plasma frequency, $\tilde{\omega}_{\mathrm{p}}$. We also neglect possible small losses in a dielectric substrate, assuming that $\varepsilon_{\mathrm{d}}$ is real and positive. Then, $\varepsilon_{\mathrm{e}}$ is purely imaginary for $\omega<\tilde{\omega}_{\mathrm{p}}$. Therefore, a film consisting of loss-free metal and dielectric grains is absorptive for $\omega<\tilde{\omega}_{\mathrm{p}}$. The effective absorption in a loss-free film means that the electromagnetic energy is stored in the system and thus the local fields could increase unlimitedly. In reality, the local fields in a metal film are, of course, finite because of the losses. If the losses are small, one anticipates very strong field fluctuations. To calculate Rayleigh, Raman scattering and various nonlinear effects in a semicontinuous metal film, one needs to know the field and current distributions in the film.

\subsection{Numerical model}

There exist now very efficient numerical methods for calculating the effective conductivity of composite materials (see [12,20]), but they typically do not allow calculations of the field distributions. Here we describe a new computer approach [36-38] based on the real space renormalization group (RSRG) method suggested by Reynolds et al. [83] and extended to study the conductivity [101] and permeability of oil reservoirs [102]. The method [36-38] follows the approach used by Aharony [102].

This approach can be adopted for finding the field distributions in the following way. First, we generate a square lattice of the $L-R$ (metal) and $C$ (dielectric) bonds, using a random number generator. As seen in Fig. 7, such lattice can be considered as a set of the "corner" elements. One of such element is labeled as (ABCDEFGH), in Fig. 7a. In the first stage of the RSRG procedure, each of these elements is replaced by the two Wheatstone bridges, as shown in Fig. 7b. After this transformation, the initial square lattice is converted to another square lattice, with the distance between the sites twice larger and with each bond between the two nearest neighboring sites being the Wheatstone bridge. Note that there is a one-to-one correspondence between the " $x$ " bonds in the initial lattice and the " $x$ " bonds in the " $x$ " directed bridges of the transformed lattice, as seen in Fig. 7. The same one-to-one correspondence exists also between the " $y$ " bonds. The transformed lattice is also a square lattice, and we can again apply to it the RSRG transformation. We continue this procedure until the size $l$ of the system is reached. As a result, instead of the initial lattice, we have two large Wheatstone bridges in the " $x$ " and " $y$ " directions. Each of them has a hierarchical structure consisting of bridges with the sizes from 2 to $l$. Because the one-to-one correspondence is preserved at each step of the transformation, the correspondence also exists between the elementary bonds of the transformed lattice and the bonds of the initial lattice.

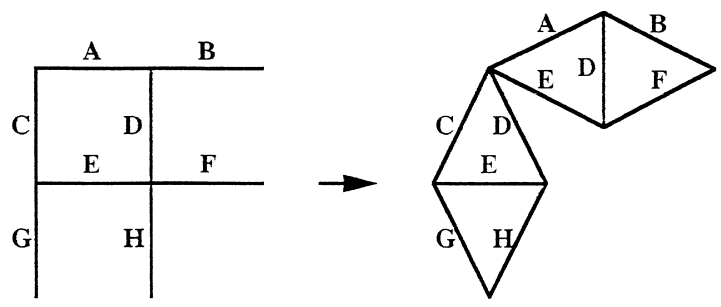

Fig. 7. The real space renormalization scheme. 
After using the RSRG transformation, we apply an external field to the system and solve the Kirchhoff equations to determine the fields and the currents in all the bonds of the transformed lattice. Due to the hierarchical structure of the transformed lattice, these equations can be solved exactly. Then, we use the one-to-one correspondence between the elementary bonds of the transformed lattice and the bonds of the initial square lattice to find the field distributions in the initial lattice as well as its effective conductivity. The number of operations to get the full distributions of the local fields is proportional to $l^{2}$ (to be compared with $l^{7}$ operations needed in the transform-matrix method [12] and $l^{3}$ operations needed in the Frank-Lobb algorithm [103]; none of these methods give the local field distributions). With our method, it takes only a few minutes to calculate the effective conductivity and field distributions in a system $1000 \times 1000$ using a PC.

The RSRG procedure is certainly not exact since the effective connectivity of the transformed system does not repeat exactly the connectivity of the initial square lattice. To check the accuracy of the RSRG, we solved the 2D percolation problem using this method. Namely, we calculated the effective parameters of a two-component composite with the real metallic conductivity, $\sigma_{\mathrm{m}}$, much larger than the real conductivity, $\sigma_{\mathrm{d}}$, of the dielectric component, $\sigma_{\mathrm{m}} \gg \sigma_{\mathrm{d}}$. We obtained the percolation threshold $p_{c}=0.5$ and the effective conductivity at the percolation threshold that is very close to $\sigma\left(p_{c}\right)=\sqrt{\sigma_{\mathrm{m}} \sigma_{\mathrm{d}}}$. These results coincide with the exact ones for 2D composites [100]. This is not surprising since the RSRG procedure preserves the self-duality of the initial system. The critical exponents obtained by the RSRG are also close to the known values of the exponents from the percolation theory [12]. Therefore, we believe that the local fields we obtain here are close to the actual ones.

All numerical results for the local field distribution and various linear and nonlinear optical effects for semicontinuous metal films, presented in this paper, have been obtained by the above method. The Drude formula 2.1 for metal dielectric functions was used and the optical constants for metals were taken from [104]. For silver, the following parameters were used in Eq. (2.1): the interband-transition contribution $\varepsilon_{\mathrm{b}}=5$, the plasma frequency $\omega_{\mathrm{p}}=9.1 \mathrm{eV}$, and the relaxation frequency $\omega_{\tau}=0.021 \mathrm{eV}$; for gold: the interband-transition contribution $\varepsilon_{\mathrm{b}}=6.5$, the plasma frequency $\omega_{\mathrm{p}}=9.3 \mathrm{eV}$, and the relaxation frequency $\omega_{\tau}=0.03 \mathrm{eV}$. The films were supposed to be deposited on a glass substrate with the dielectric constant $\varepsilon_{\mathrm{d}}=2.2$ for all numerical simulations discussed in this paper.

\subsection{Field distributions on semicontinuous metal films}

As mentioned, we model a film by a square lattice consisting of metallic bonds with conductivity $\sigma_{\mathrm{m}}=-\mathrm{i} \varepsilon_{\mathrm{m}} \omega / 4 \pi$ ( $L-R$ bonds) and concentration $p$, and dielectric bonds with conductivity $\sigma_{\mathrm{d}}=-\mathrm{i} \varepsilon_{\mathrm{d}} \omega / 4 \pi$ and concentration $1-p$ ( $C$ bonds). The applied field $E_{0}$ is set to be equal unity $E_{0}=1$, whereas the local fields inside the system are complex quantities. The dielectric constant of silver grains has the form of Eq. (2.1) with parameters discussed in last subsection. Below, we still use $\varepsilon_{\mathrm{d}}=2.2$ typical for a glass. In Fig. 1 we show the field distributions $\left|E(\boldsymbol{r}) / E_{0}\right|^{2}$ for the plasmon resonance frequency $\omega=\omega_{\mathrm{r}}$ that corresponds to the condition $\operatorname{Re}\left(\varepsilon_{\mathrm{m}}\left(\omega_{\mathrm{r}}\right)\right)=-\varepsilon_{\mathrm{d}}$. The value of the frequency $\omega_{\mathrm{r}}$ is slightly below the renormalized plasma frequency $\tilde{\omega}_{\mathrm{p}}$ defined above in Eq. (2.2). For silver particles the resonance condition fulfilled at wavelength $\lambda \approx 0.4 \mu \mathrm{m}$. The frequency $\omega_{\mathrm{r}}$ gives 
the resonance of an isolated metal particle. (For a 2D, i.e., $z$-independent problem, particles can be thought of as infinite in the $z$-direction cylinders that resonate, in the quasistatic approximation, at the frequency $\omega=\omega_{\mathrm{r}}$ corresponding to the condition $\varepsilon_{\mathrm{m}}\left(\omega_{\mathrm{r}}\right)=-\varepsilon_{\mathrm{d}}$, for the field polarized in the $x, y$-plane). The results are presented for various metal fractions $p$. For $p=0.001$ metal grains practically do not interact so that all the peaks are almost of the same height and indicate the locations of metal particles. Note that similar distribution is obtained for $p=0.999$ when the role of metal particles is played by the dielectric voids. For $p=0.1$ and, especially, for $p=0.5$, metal grains form clusters of strongly interacting particles. These clusters resonate at different frequencies (than that for an isolated particle), therefore, for the chosen frequency the field peaks are smaller, on average, than those for the isolated particles, and the height distribution is very inhomogeneous. Note that spatial scale for the local field distribution is much larger than the metal grain size $a$ that is chosen to be unity for all the figures. Therefore, the main assumption of the effective medium theory $[13,50-55]$ that the local fields are the same for all metal grains fails for the frequencies of the plasmon resonance and nonvanishing concentrations $p$. We emphasize a strong resemblance in the field distributions for $p$ and $1-p$ (cf. Fig. 1a and $\mathrm{g}, \mathrm{b}$ and $\mathrm{f}, \mathrm{c}$ and e).

For larger wavelengths, a single metal grain is off the plasmon resonance. Nevertheless, as one can see from Fig. 2a-d, the local field fluctuations are even larger than those at the plasmon resonance frequency. At these wavelengths, clusters of the conducting particles (rather than individual particles) resonate with the external field oscillations. Therefore, it is not surprising that the local field distributions are quite different from those in Fig. 1. In Fig. 2, we show the field distributions at the percolation threshold $p=p_{c}=0.5$ for different wavelengths, namely, (2a): $\lambda=0.5 \mu \mathrm{m},(2 \mathrm{~b}): \lambda=1.5 \mu \mathrm{m},(2 \mathrm{c}): \lambda=10 \mu \mathrm{m}$, and $(2 \mathrm{~d}): \lambda=20 \mu \mathrm{m}$. Note that the field intensities in peaks increase with $\lambda$, reaching very high values, $\sim 10^{5} E_{0}$; the peak spatial separations increase with $\lambda$ as well. These results are also in contradiction with the effective medium theory that predicts strong field fluctuations [34] in the vicinity of plasmon resonance frequency $\omega_{\mathrm{r}}$ only. In the previous section, we have presented a scaling theory for the field distributions that explains, at least qualitatively, all peculiarities of the above results.

The first experiment on the field distribution in the semicontinuous metal films [39] had been done in the microwave range and will be discussed in Section 7. For the visible spectral range the local field distribution qualitatively similar to the calculated (see Figs. 1 and 2) has been experimentally obtained using scanning near-field optical microscope (SNOM) providing subwavelength resolution $[46,58]$. An SNOM was operating in the tapping mode, so that the detected local signals were averaged over the tip-surface separations ranging between 0 and $100 \mathrm{~nm}$. Because of this and the finite size of the tip, the detected field intensities were by two to three orders of magnitude less than the actual field right on the film surface (that can be probed, for example, by surface-adsorbed molecules). Aside this, the detected field distribution shown in Fig. 8 is similar to that predicted by theory (Figs. 1 and 2). When the SNOM averaging effect is taken into account in simulations there is very good agreement between the calculations and experiments as seen in Fig. 8.

The near-field spectroscopy of percolation films was also performed in $[46,58]$, by parking an SNOM tip at different points of the surface and varying the wavelength. This local nanospectroscopy allows one to determine the local resonances of $\mathrm{nm}$-size areas right underneath the tip; the nano-structures at different points resonate at different $\lambda$ leading to different local near-field spectra. The spectra characterize $\lambda$-dependence of the field hot spots associated with the localized s.p. modes. 

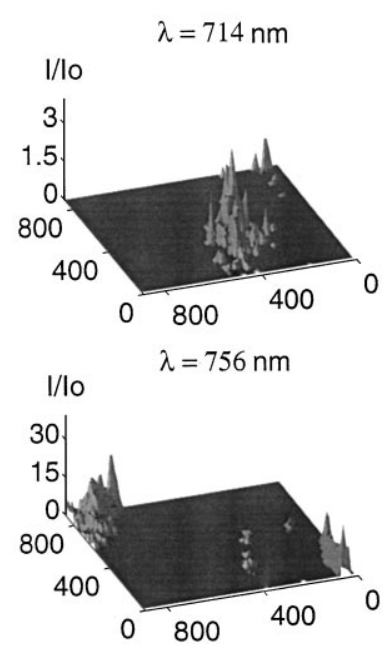

(a)
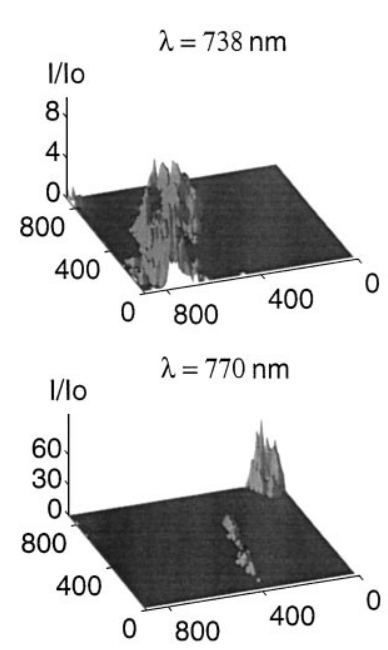
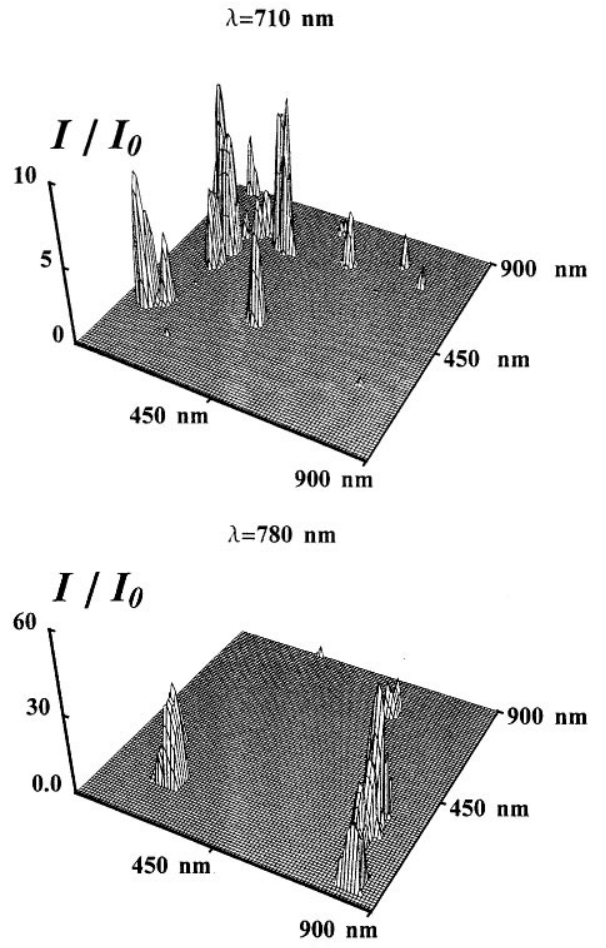

(b)

Fig. 8. Experimental (a) and calculated (b) SNOM images of the localized optical excitations in a percolation gold-on-glass film for different wavelengths $\lambda$.

In Fig. 9 we show the measured and calculated near-field spectra taken at different points of the film. Again, there is qualitative agreement between theory and experiment. The spectra consist of several peaks $\sim 10 \mathrm{~nm}$ in width, and they depend markedly on spatial location of the point where the near-field tip is parked. Even as small shift in space as $100 \mathrm{~nm}$ results in different spectra, which is a strong evidence of the s.p.-mode localization. We note that for continuous metal (or dielectric) films neither sub- $\lambda$ hot spots nor their local spectra can be observed, because, in this case, optical excitations are delocalized.

In conclusion, the near-field imaging and spectroscopy of random metal-dielectric films near percolation suggests localization of optical excitations in small $\mathrm{nm}$-scale hot spots. The observed pattern of the localized modes and their spectral dependences are in agreement with theoretical predictions and numerical simulations. The hot spots of a percolation film represent very large local fields (fluctuations); spatial positions of the spots strongly depend on the light frequency. Near-field spectra observed and calculated at various points of the surface consist of several spectral resonances whose spectral locations depend on the probed site of the sample. All these features are only observable in the near zone. In the far zone, one observes images and spectra in which the hot spots and the spectral resonances are averaged out. The local field enhancement is large, which is especially important for nonlinear processes of the $n$th order proportional to the 

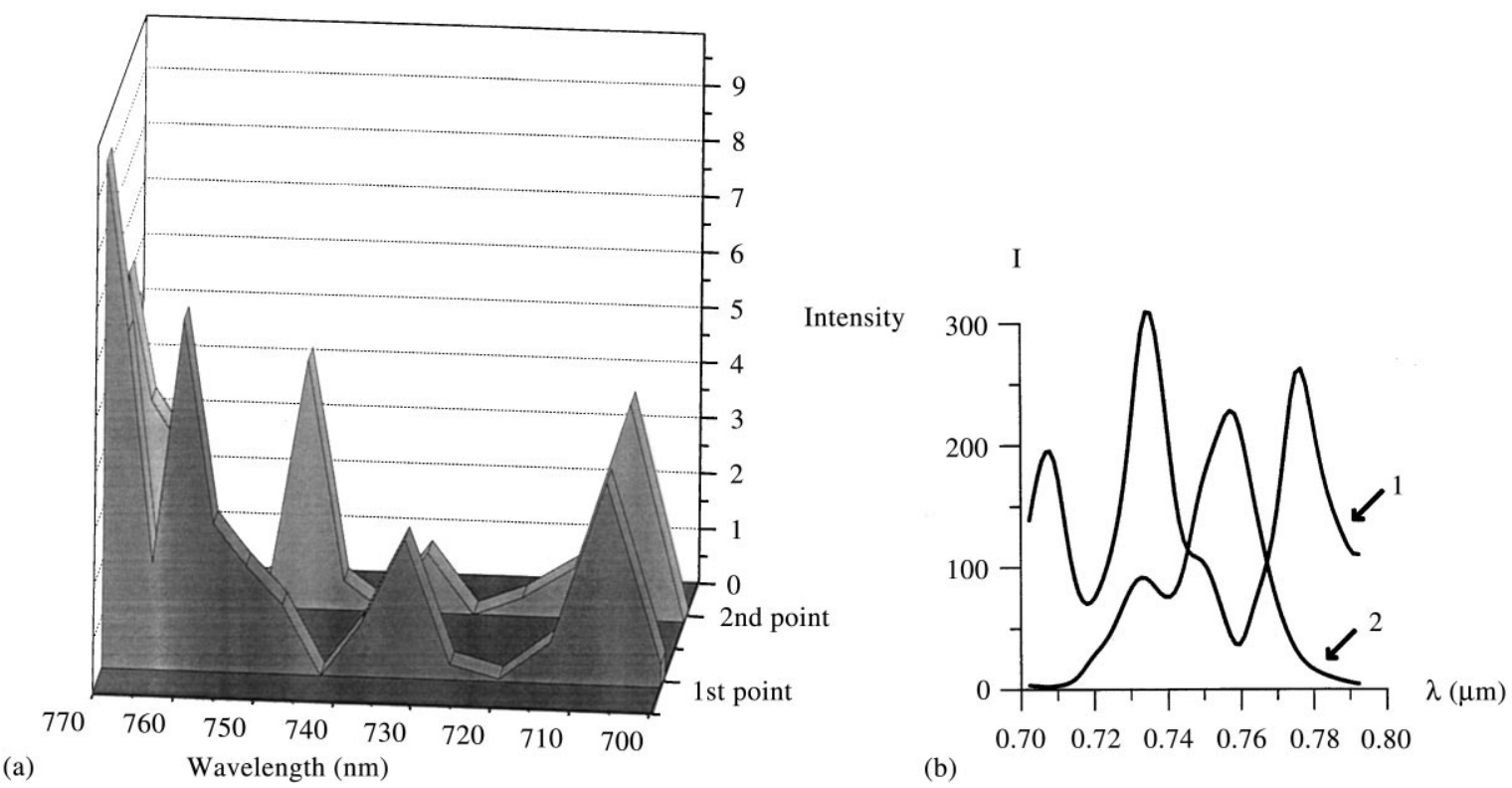

Fig. 9. Experimental (a) and calculated (b) near-field spectra at different spatial locations (100 nm apart) of the film. (Arbitrary intensity units are used.)

enhanced local fields to the $n$th power. This opens a fascinating possibility for nonlinear near-field spectroscopy of single nanoparticles and molecules.

\section{Anomalous light scattering from semicontinuous metal films}

In this section we consider quantitatively the spatial distribution of the local field fluctuations and light scattering induced by these fluctuations. Numerical as well as analytical results are presented for 2D semicontinuous metal films that, as above (see Section 3), are modeled as a 2D $L-C$ lattice where capacitors $C$ stand for the dielectric grains that have dielectric constant $\varepsilon_{\mathrm{d}}$ while the inductances $L$ represent the metallic grains that have dielectric constant $\varepsilon_{\mathrm{m}}$. The resonance frequency $\omega_{\mathrm{r}}$, corresponding to the condition $\varepsilon_{\mathrm{m}}^{\prime}\left(\omega_{\mathrm{r}}\right)=-\varepsilon_{\mathrm{d}}$ is considered first. For a Drude metal, when metal dielectric constant $\varepsilon_{\mathrm{m}}$ is given by Eq. (2.1), this condition is fulfilled at the frequency

$$
\omega_{\mathrm{r}}=\omega_{\mathrm{p}} \sqrt{1 /\left(\varepsilon_{\mathrm{b}}+\varepsilon_{\mathrm{d}}\right)-\left(\omega_{\tau} / \omega_{\mathrm{p}}\right)^{2}} \simeq \omega_{\mathrm{p}} / \sqrt{\varepsilon_{\mathrm{b}}+\varepsilon_{\mathrm{d}}},
$$

where it is taken into account that the relaxation rate $\omega_{\tau}=1 / \tau \ll \omega_{\mathrm{p}}$ for a typical metal. Then metal dielectric function takes value $\varepsilon_{\mathrm{m}}\left(\omega_{\mathrm{r}}\right)=\varepsilon_{\mathrm{d}}(-1+\mathrm{i} \kappa)$, where the loss factor $\kappa$ is equal to $\kappa \simeq\left(1+\varepsilon_{\mathrm{b}} / \varepsilon_{\mathrm{d}}\right) \omega_{\tau} / \omega_{\mathrm{r}} \ll 1$. Remind that for silver or gold the ratio $\omega_{\tau} / \omega_{\mathrm{r}} \simeq 10^{-2}$. Since distribution of the local field does not change when bond conductances are multiplied by the same factor it is convenient to consider the lattice where a bond conductance takes value $\Sigma_{\mathrm{m}}=-1+\mathrm{i} \kappa$ with probability $p$ ( $L$ bonds) and $\Sigma_{\mathrm{d}}=1$ with probability $1-p$ ( $C$ bonds). Since the absolute values of 
$\Sigma_{\mathrm{d}}$ and $\Sigma_{\mathrm{m}}$ are very close, the standard method, using the percolation theory scaling approach $[12,20,24,64]$ and based on the assumption of large difference between the two components conductivities, cannot be used, even to estimate the spatial distribution of the field in the system. This is the reason why computer simulation has been used in the works $[36,37,44]$.

For numerical calculations, the RSRG method described in Section 3 is applied to a system of size $1024 \times 1024$. The concentrations of $C$ and $L$ elements are equal to $p=p_{c}=0.5$ and frequency $\omega$ is equal to the resonance frequency $\omega=\omega_{\mathrm{r}}$. The electric field was calculated in all the bonds for the loss parameters $\kappa$ from $10^{-1}$ to $10^{-4}$. The external field is assumed to be one $\left(E_{0}=1\right)$ while the local fields inside the system are complex quantities.

The distribution function of the field intensity $I(\boldsymbol{r})=|E(\boldsymbol{r})|^{2}$ is close to the well-known log-normal distribution and the field intensity is distributed over many orders of magnitude, even for a loss parameter $\kappa=10^{-1}$. For the parameter $\kappa=10^{-4}$, the intensity $I$ is almost uniformly distributed from zero up to $10^{4}$. The average intensity $I_{\mathrm{av}}=\left|E_{0}\right|^{2} M_{2}$ increases as $I_{\mathrm{av}} \propto \kappa^{-1}$ when the loss parameter $\kappa$ is decreased, which is in agreement with scaling Eq. (2.33). Note that the same result $I_{\mathrm{av}} \propto \kappa^{-1}$ have been conjecture for fractals consisting of small metal particles (see, for example, Ref. [25]).

It is clear that the field fluctuations, shown in Figs. 1, 2 and 10 can lead to the enhanced light scattering from the film. The Rayleigh scattering from metal fractals was studied in Ref. [105].

It is worth noting that the fluctuations considered here and the corresponding light scattering are not linked to the fractal nature of metal clusters but to the distribution of local resonances in a disordered metal-dielectric film, which is homogeneous on a macroscopical scale. It appears that the local intensity of the electric field is strongly correlated in space and that the distribution is dominated by the field correlation length $\xi_{\mathrm{e}}$ introduced by Eqs. (2.24) and (2.40). The field correlation length may be defined as the length scale at which the field fluctuations become small. One can then define a critical exponent related to the divergence of this correlation length as the $L$ (metallic) component becomes loss-free

$$
\xi_{\mathrm{e}} \propto \kappa^{-v_{\mathrm{e}}},
$$

where $v_{\mathrm{e}}$ is a critical exponent. This exponent has been calculated using several numerical methods [36,37] based on RSRG method described in Section 3. The values obtained for $v_{\mathrm{e}}$ are in the range $0.45 \pm 0.05$. The scaling theory presented in Section 2 gives $v_{\mathrm{e}}=1 / d$ [see Eqs. (2.24) and (2.40)], where $d$ is the space dimension. The result $v_{\mathrm{e}}=1 / d$ has been conjectured for the first time by Stinchcombe and Hesselbo [106]. Note that the dimension $D$ connected to the Wheatstone bridge transformation in the RSRG method is given by $D=\log 5 / \log 2$, which gives here $1 / D=0.43$, very close to the numerical result $v_{\mathrm{e}}=0.45 \pm 0.05$. For small loses at resonance, the correlation length $\xi_{\mathrm{e}}$ is the only relevant characteristic length of the system at percolation threshold since the contrast $\left|\varepsilon_{\mathrm{m}}\right| / \varepsilon_{\mathrm{d}}$ is close to one. In this case, the percolation dynamic correlation length defined by Eqs. (2.34) as $l_{\mathrm{r}}=a\left(\left|\varepsilon_{\mathrm{m}}\right| / \varepsilon_{\mathrm{d}}\right)^{v /(t+s)}$ [20] reduces to the size of a single bond and is irrelevant for the problem.

As the frequency is varied from its resonant value $\omega_{\mathrm{r}}$ a crossover between the region characterized by $\xi_{\mathrm{e}}$ and $l_{\mathrm{r}}$ is observed clearly at lengths smaller than the percolation correlation length $\xi_{\mathrm{p}}[37]$. 


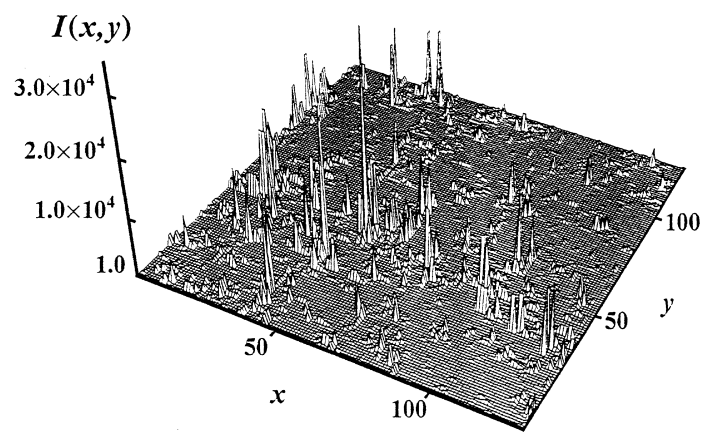

Fig. 10. Intensity of the electric field $I(\boldsymbol{r})=|\boldsymbol{E}(\boldsymbol{r})|^{2}$ in gold semicontinuous metal films at wavelength $\lambda=1.5 \mu \mathrm{m}, p=p_{c}$. The amplitude of the incident wave $\left|\boldsymbol{E}_{0}\right|^{2}$ is set equal to one.

\subsection{Rayleigh scattering}

To calculate theoretically and numerically the anomalous scattering resulting from the spatial correlations of the local field fluctuations, the values of the dielectric constant for gold were used to estimate semiquantitatively enhancement of the scattering. Fig. 10 shows the intensity of the local electric field $I(\boldsymbol{r})=|E(\boldsymbol{r})|^{2}$ (where $\boldsymbol{r}=\{x, y\}$ is a radius vector in the film plane) obtained by the RSRG method, presented in Section 3, for a gold semicontinuous film consisting of small particles at $p=p_{c}$ for $\lambda=1.5 \mu \mathrm{m}$. One can see the intensity fluctuations of more than four orders of magnitude over the film.

We consider now Rayleigh scattering induced by the giant field fluctuations [44]. The usual procedure leading to expressions for the critical opalescence [65] can be implemented to find the anomalous scattering from the metal-dielectric films. Suppose a semicontinuous film is illuminated by a wave normal to the film plane. The gaps between metal grains are filled by dielectric material of the substrate. Therefore, the film can be considered as a two-dimensional array of metal and dielectric grains that are distributed over the plane. The incident electromagnetic wave excites the surface currents $\boldsymbol{j}$ in the film. Let us consider the electromagnetic field induced by these currents at some distant point $\boldsymbol{R}$. The coordinate origin is chosen somewhere in the film. Then a contribution to the vector potential $\boldsymbol{A}(\boldsymbol{R})$ of the scattered field \{magnetic field $\boldsymbol{H}(\boldsymbol{R})=[\nabla \times \boldsymbol{A}(\boldsymbol{R})]$ \} arising from the surface current $j(r)$ is equal to

$$
\boldsymbol{A}(\boldsymbol{R}, \boldsymbol{r}) \mathrm{d} \boldsymbol{r}=\frac{j(\boldsymbol{r})}{c} \frac{\exp (\mathrm{i} k|\boldsymbol{R}-\boldsymbol{r}|)}{|\boldsymbol{R}-\boldsymbol{r}|} \mathrm{d} \boldsymbol{r}
$$

where $k=\omega / c$ is a wave vector. The vector potential $\boldsymbol{A}(\boldsymbol{R})$ of the total scattered field is equal to $\boldsymbol{A}(\boldsymbol{R})=\int \boldsymbol{A}(\boldsymbol{R}, \boldsymbol{r}) \mathrm{d} \boldsymbol{r}$, where the integration is over the entire film area. In experiments, the dimensions of the film are typically small compared to the distance $R(r \ll R)$. Therefore, the term in the exponent of Eq. (4.3) can be expanded in series of the ratio $r / R$; this gives $\mathrm{i} k|\boldsymbol{R}-\boldsymbol{r}| \cong \mathrm{i} k R-\mathrm{i} k(\boldsymbol{n} \cdot \boldsymbol{r})$, where $\boldsymbol{n}$ is the unit vector in the direction of $\boldsymbol{R}$. The distance $r$ is also neglected in comparison with $R$ in the denominator of Eq. (4.3). Thus the equations for the magnetic $\boldsymbol{H}$ and electric $\boldsymbol{E}$ fields at 
a distant point $\boldsymbol{R}$ take the following form:

$$
\begin{aligned}
& \boldsymbol{H}(\boldsymbol{R})=[\nabla \times \boldsymbol{A}(\boldsymbol{R})] \cong \frac{\mathrm{i} k \exp (\mathrm{i} k R)}{c R} \int[\boldsymbol{n} \times \mathbf{j}(\boldsymbol{r})] \exp [-\mathrm{i} k(\boldsymbol{n} \cdot \boldsymbol{r})] \mathrm{d} \boldsymbol{r}, \\
& \boldsymbol{E}(\boldsymbol{R})=\frac{\mathrm{i}}{k}[\nabla \times \boldsymbol{H}(\boldsymbol{R})] \cong \frac{-\mathrm{i} k \exp (\mathrm{i} k R)}{c R} \int[\boldsymbol{n} \times[\boldsymbol{n} \times \mathbf{j}(\boldsymbol{r})]] \exp [-\mathrm{i} k(\boldsymbol{n} \cdot \boldsymbol{r})] \mathrm{d} \boldsymbol{r},
\end{aligned}
$$

where the integrations are over the film area. It follows from Eqs. (4.4) that the magnetic field $\boldsymbol{H}(\boldsymbol{R})$ is perpendicular to the electric field $\boldsymbol{E}(\boldsymbol{R})$ and their absolute values are equal to each other $|\boldsymbol{E}(\boldsymbol{R})|=|\boldsymbol{H}(\boldsymbol{R})|$, which means that the scattered field can be considered locally as a plane wave, when the distance from the film is large. The total intensity $S_{\mathrm{t}}$ of the light scattered in the direction $\boldsymbol{n}=\boldsymbol{R} / R$ is equal to

$$
\begin{aligned}
S_{\mathrm{t}}(\boldsymbol{n}) & =\frac{c}{4 \pi} R^{2} \frac{1}{2} \operatorname{Re}\left\langle\left[\boldsymbol{E}(\boldsymbol{R}) \times \boldsymbol{H}^{*}(\boldsymbol{R})\right]\right\rangle \\
& =\frac{c}{8 \pi} R^{2}\left\langle\boldsymbol{E}(\boldsymbol{R}) \cdot \boldsymbol{E}^{*}(\boldsymbol{R})\right\rangle=\frac{c}{8 \pi} R^{2}\left\langle\boldsymbol{H}(\boldsymbol{R}) \cdot \boldsymbol{H}^{*}(\boldsymbol{R})\right\rangle \\
& \left.=\frac{c}{8 \pi} \frac{k^{2}}{c^{2}} \int\left[\boldsymbol{n} \times \boldsymbol{j}\left(\boldsymbol{r}_{1}\right)\right] \cdot\left[\boldsymbol{n} \times \boldsymbol{j}^{*}\left(\boldsymbol{r}_{2}\right)\right]\right\rangle \exp \left[\mathrm{i} k \boldsymbol{n} \cdot\left(\boldsymbol{r}_{1}-\boldsymbol{r}_{2}\right)\right] \mathrm{d} \boldsymbol{r}_{1} \mathrm{~d} \boldsymbol{r}_{2},
\end{aligned}
$$

where "*" denotes, as above, complex conjugation and the angular brackets stand for the ensemble averaging. Note that the semicontinuous metal films considered here are much larger than any characteristic intrinsic spatial scale, such as the field correlation length $\xi_{\mathrm{e}}$. Therefore, the ensemble average can be included in the integrations over the film area in Eqs. (4.5) without changing the result. It is assumed, for simplicity, that the incident light is natural (unpolarized) and that its direction is perpendicular to the film plane. Then the product $\left\langle\left[\boldsymbol{n} \times \boldsymbol{j}\left(\boldsymbol{r}_{1}\right)\right] \cdot\left[\boldsymbol{n} \times \boldsymbol{j}^{*}\left(\boldsymbol{r}_{2}\right)\right]\right\rangle$ in Eqs. (4.5) should be averaged over the polarizations of the incident wave, which gives $\left\langle\boldsymbol{j}\left(\boldsymbol{r}_{1}\right) \cdot \boldsymbol{j}^{*}\left(\boldsymbol{r}_{2}\right)\right\rangle\left(1-\sin ^{2} \theta / 2\right)$, where $\theta$ is the angle between the direction $\boldsymbol{n}$ and normal to the film plane.

The replacing of the local currents $\boldsymbol{j}(\boldsymbol{r})$ by their averaged values $\langle\boldsymbol{j}(\boldsymbol{r})\rangle$ in Eq. (4.5) gives the specular scattering $S_{\mathrm{s}}$. The scattering in all other directions is obtained as

$$
\begin{aligned}
S(\theta) & =S_{\mathrm{t}}-S_{\mathrm{s}} \\
& =\frac{c}{8 \pi} \frac{k^{2}}{c^{2}}\left(1-\frac{\sin ^{2} \theta}{2}\right) \int\left[\left\langle\boldsymbol{j}\left(\boldsymbol{r}_{1}\right) \cdot \boldsymbol{j}^{*}\left(\boldsymbol{r}_{2}\right)\right\rangle-|\langle\boldsymbol{j}\rangle|^{2}\right] \exp \left[\mathrm{i} k \boldsymbol{n} \cdot\left(\boldsymbol{r}_{1}-\boldsymbol{r}_{2}\right)\right] \mathrm{d} \boldsymbol{r}_{1} \mathrm{~d} \boldsymbol{r}_{2} .
\end{aligned}
$$

There is a natural correlation length $\xi_{\mathrm{e}}$ [see Eqs. (2.24), (2.40), and (4.2)] for the local field fluctuations and, therefore, for the current-current correlations. If this correlation length is much smaller than the wavelength of the incident light $\xi_{\mathrm{e}} \ll \lambda=2 \pi / k$, Eq. (4.6) is simplified by replacing the exponent by unity $\left(\exp \left(\mathrm{i} k \boldsymbol{n} \cdot\left(\boldsymbol{r}_{1}-\boldsymbol{r}_{2}\right)\right) \approx 1\right)$. This gives

$$
S(\theta)=\frac{c}{8 \pi} \frac{k^{2}}{c^{2}}\left(1-\frac{\sin ^{2} \theta}{2}\right)|\langle\boldsymbol{j}\rangle|^{2} \int\left[\frac{\left\langle\boldsymbol{j}\left(\boldsymbol{r}_{1}\right) \cdot \boldsymbol{j}^{*}\left(\boldsymbol{r}_{2}\right)\right\rangle}{|\langle\boldsymbol{j}\rangle|^{2}}-1\right] \mathrm{d} \boldsymbol{r}_{1} \mathrm{~d} \boldsymbol{r}_{2} .
$$


Since the macroscopically homogeneous and isotropic films are considered the current-current correlations $\left\langle\boldsymbol{j}\left(\boldsymbol{r}_{1}\right) \cdot \boldsymbol{j}^{*}\left(\boldsymbol{r}_{2}\right)\right\rangle$ depend only on the distance $r=\left|\boldsymbol{r}_{2}-\boldsymbol{r}_{1}\right|$ between the currents. It is convenient to introduce the correlation function

$$
G(r)=\frac{\left\langle\boldsymbol{j}\left(\boldsymbol{r}_{1}\right) \cdot \boldsymbol{j}^{*}\left(\boldsymbol{r}_{2}\right)\right\rangle}{|\langle\boldsymbol{j}\rangle|^{2}}-1=\frac{\operatorname{Re}\left\langle\boldsymbol{j}(0) \cdot \boldsymbol{j}^{*}(\boldsymbol{r})\right\rangle}{|\langle\boldsymbol{j}\rangle|^{2}}-1 .
$$

Substituting this correlation function in Eq. (4.7) and replacing the integrations over coordinates $\boldsymbol{r}_{1}$ and $\boldsymbol{r}_{2}$ by integrations over $\boldsymbol{r}=\boldsymbol{r}_{2}-\boldsymbol{r}_{1}$ and $\boldsymbol{r}^{\prime}=\left(\boldsymbol{r}_{2}+\boldsymbol{r}_{1}\right) / 2$, the following equation is obtained for the intensity of the scattered light:

$$
S(\theta)=A \frac{c}{8 \pi} \frac{k^{2}}{c^{2}}\left(1-\frac{\sin ^{2} \theta}{2}\right)|\langle\boldsymbol{j}\rangle|^{2} 2 \pi \int_{0}^{\infty} G(r) r \mathrm{~d} r,
$$

where $A$ is the film area. The intensity of the scattered light is compared with the integral intensity (power) of the incident light $I_{0}=A(c / 8 \pi)\left|\boldsymbol{E}_{0}\right|^{2}$, where $\boldsymbol{E}_{0}$ is the amplitude of the incident wave. For the normal incident light, the average electric field in the film $\langle\boldsymbol{E}\rangle$ is equal to $\langle\boldsymbol{E}\rangle=T \boldsymbol{E}_{0}$, where $T$ is the transmittance of the film (see discussion in Ref. [38]). Note that for semicontinuous metallic films at $p=p_{c}$ the transmittance $|T|^{2} \approx 0.25$ in a wide spectral range from the visible to the far infrared spectral range [85]. The average surface current $\langle\boldsymbol{j}\rangle$ is related to the average electric field $\langle\boldsymbol{E}\rangle$ through the Ohm's law; in thin films it takes the following form $\langle\boldsymbol{j}\rangle=a \sigma_{\mathrm{e}}\langle\boldsymbol{E}\rangle=a \sigma_{\mathrm{e}} T \boldsymbol{E}_{0}$, where $\sigma_{\mathrm{e}}=-\mathrm{i} \varepsilon_{\mathrm{e}} \omega /(4 \pi)$ is the effective conductivity, and thickness of the film is approximated by the size $a$ of a metal grain.

By substituting $\langle\boldsymbol{j}\rangle=\sigma_{\mathrm{e}} a T \boldsymbol{E}_{0}$ in Eq. (4.9), the ratio of the scattering intensity $S(\theta)$ to the total intensity of the incident light $I_{0}$ can be obtained. This ratio is independent of a film geometry:

$$
\tilde{S}(\theta)=\frac{S(\theta)}{I_{0}}=\frac{2 \pi(k a)^{2}}{c^{2}}\left(1-\frac{\sin ^{2} \theta}{2}\right)\left|T \sigma_{\mathrm{e}}\right|^{2} \int_{0}^{\infty} G(r) r \mathrm{~d} r,
$$

which can be rewritten as

$$
\tilde{S}(\theta)=\frac{(k a)^{4}}{8 \pi}\left(1-\frac{\sin ^{2} \theta}{2}\right)\left|T \varepsilon_{\mathrm{e}}\right|^{2} \frac{1}{a^{2}} \int_{0}^{\infty} G(r) r \mathrm{~d} r .
$$

It follows from this equation that the portion of the incident light that is not reflected, transmitted or adsorbed, but is scattered from the film is equal to

$$
S_{\text {tot }}=2 \pi \int \tilde{S}(\theta) \sin \theta \mathrm{d} \theta=\frac{(k a)^{4}}{3}\left|T \varepsilon_{\mathrm{e}}\right|^{2} \frac{1}{a^{2}} \int_{0}^{\infty} G(r) r \mathrm{~d} r .
$$

Eqs. (4.11) and (4.12) have a transparent physical meaning. The anomalous scattering like Rayleigh scattering is inversely proportional to the fourth power of the wavelength $\tilde{S} \propto S_{\text {tot }} \propto(a k)^{4} \propto(a / \lambda)^{4}$, and it is much enhanced due to spatial current-current (field-field) correlations described by the correlation function $G(r)$ in Eqs. (4.9)-(4.12). The function $G(r)$ can have different behaviors for different frequencies. The factor $\left|T \varepsilon_{\mathrm{e}}\right|^{2}$ in Eq. (4.12) also depends on the frequency and achieves large values $\left|T \varepsilon_{\mathrm{e}}\right|^{2} \gg 1$ in the infrared spectral range. 


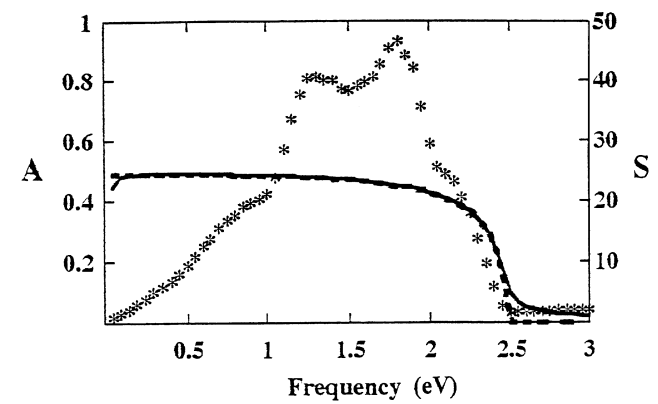

Fig. 11. Absorptance of gold semicontinuous films at $p=p_{c}$ - continuous line; the same but film is loss free - dash line; $*-S=S_{\text {tot }} / 4 \pi-$ average scattering from the film into the unit solid angle $\left(p=p_{c}\right)$.

The scattering given by Eq. (4.12) can be compared with the scattering in the case when the metal grains interact with the electromagnetic field independently. The cross-section $\sigma_{\mathrm{R}}$ of Rayleigh scattering from a single metal grain can be estimated as $\sigma_{\mathrm{R}}=(8 \pi / 3)(k a)^{4} a^{2}$ for $\left|\varepsilon_{\mathrm{m}}\right| \gg 1$ [82]. The portion of the light which would be scattered if the grains were independent is equal then to $S_{\text {tot }}^{\mathrm{R}} \simeq p(8 / 3)(k a)^{4}$. Assuming $p=1 / 2$, the following estimate is obtained for the enhancement $g$ of the scattering due to the field fluctuations:

$$
g=\frac{S_{\mathrm{tot}}}{S_{\mathrm{tot}}^{\mathrm{R}}} \sim \frac{\left|T \varepsilon_{\mathrm{e}}\right|^{2}}{4 a^{2}} \int_{0}^{\infty} G(r) r \mathrm{~d} r .
$$

If the integral in this equation is determined by the largest distances where field correlations are essential, i.e., $r \sim \xi_{\mathrm{e}}$, the scattering is enhanced up to infinity, when the losses vanish and $\xi_{\mathrm{e}} \rightarrow \infty$. It is the case for 2D metal-dielectric films as it is shown below. Certainly, the above formalism holds if the part of the incident wave being scattered is much less than one, $S_{\text {tot }} \ll 1$. Otherwise, it is necessary to take into account the feedback effects, i.e., the interaction of the scattered light with the film.

The function $G(r)$ for $1024 \times 1024 L-C$ system is calculated using the RSRG method, discussed in the previous section, and scattering function $S_{\text {tot }}(\omega)$ is obtained for gold semicontinuous metal film at the percolation threshold $p=p_{c}=1 / 2$. The results are shown in Figs. 11 and 13. One can see in Fig. 11 that the scattering dramatically increases for $\omega$ below $\tilde{\omega}_{\mathrm{p}}$ when real part of the metal dielectric function $\varepsilon_{\mathrm{m}}^{\prime}$ becomes negative. The scattering has a broad double peak maximum and finally drops down in the infrared spectral range, where $\omega_{\tau} \ll \omega \ll \tilde{\omega}_{\mathrm{p}}$. To understand this result let us investigate the behavior of the correlation function $G(r)$ in more detail, using numerical calculations as well as the scaling arguments discussed in Section 2.

\subsection{Scaling properties of correlation function}

The correlation function $G(r)$ was calculated for the resonance frequency $\omega_{\mathrm{r}}$ [see Eq. (4.12)] so that $\varepsilon_{\mathrm{m}}^{\prime}\left(\omega_{\mathrm{r}}\right)=-\varepsilon_{\mathrm{d}}$, and for different values of loss parameters $\kappa=\varepsilon_{\mathrm{m}}^{\prime \prime} /\left|\varepsilon_{\mathrm{m}}^{\prime}\right|[44]$. The concentration of metal grains represented by $L-R$ elements is equal to $p=p_{c}=1 / 2$. The system size is equal to $1024 \times 1024$ and results are averaged over 100 different realizations of the system for each value 


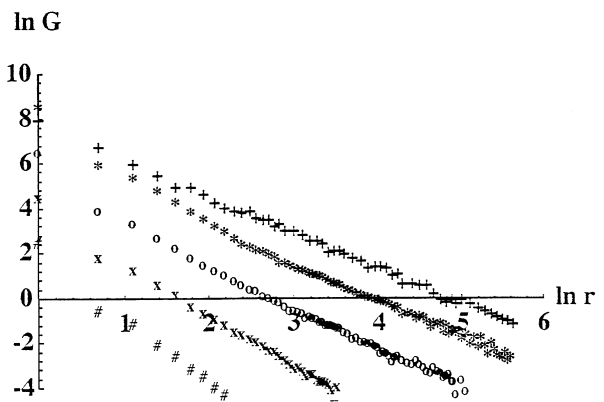

Fig. 12. Correlation function $G(r)$ for the $-\varepsilon_{\mathrm{m}}^{\prime}=\varepsilon_{\mathrm{d}}$ and different loss parameters $\kappa=\varepsilon_{\mathrm{m}}^{\prime \prime} /\left|\varepsilon_{\mathrm{m}}^{\prime}\right|: \neq-\kappa=10^{-1}$, $\times-\kappa=10^{-2}, \bigcirc-\kappa=10^{-3}, *-\kappa=10^{-4}$. The same for $H=100.0$ and $\kappa=10^{-3}-+$

of $\kappa$. The obtained function $G(r)$ is shown in Fig. 12 (the distance $r$ is measured in units of the metal grain size $a$ ). It follows from Fig. 12 that for the scales $a<r<\xi_{\mathrm{e}}$, the correlation function decays as

$$
G^{\star}(r) \sim M_{2}^{\star}(r / a)^{-(1+\eta)} \sim \kappa^{-1}(r / a)^{-(1+\eta)},
$$

where $M_{2}^{\star}$, given by Eq. (2.22), is the second moment of the local field in the system with $\varepsilon_{\mathrm{m}}^{\prime}=-\varepsilon_{\mathrm{d}}$, and the critical exponent $\eta=0.8 \pm 0.1$ is a new critical exponent that determines the spatial correlation of the local electric field. Substitution of the correlation function $G$ from Eq. (4.14) in expressions (4.11) and (4.12) for the scattering gives that the integrals diverge at the upper limit. Therefore, the scattering is determined by the values of the function $G(r)$ at large distances, where Eq. (4.14) still holds, i.e., $r \sim \xi_{\mathrm{e}}$. This suggests that the field fluctuations with spatial distances of the field correlation length $\xi_{\mathrm{e}} \gg 1$ are responsible for the anomalous scattering from semicontinuous films.

Now the dependence of scattering on the frequency of an incident electromagnetic wave is considered. First we consider frequencies just below $\tilde{\omega}_{\mathrm{p}}$ when the metal dielectric function can be estimated for a Drude metal as $\varepsilon_{\mathrm{m}}=\varepsilon_{\mathrm{m}}^{\prime}+\mathrm{i} \varepsilon_{\mathrm{m}}^{\prime \prime} \cong 2 \varepsilon_{\mathrm{b}}\left(\omega-\tilde{\omega}_{\mathrm{p}}\right) / \tilde{\omega}_{\mathrm{p}}+\mathrm{i} \varepsilon_{\mathrm{b}} \omega_{\tau} / \tilde{\omega}_{\mathrm{p}}$ [see Eq. (2.1)], i.e., $\varepsilon_{\mathrm{m}}^{\prime}<0$. The contrast $\left|\varepsilon_{\mathrm{m}}^{\prime}\right| / \varepsilon_{\mathrm{d}}$ is about unity $\left|\varepsilon_{\mathrm{m}}^{\prime}\right| / \varepsilon_{\mathrm{d}} \leq 1$ for these frequencies, while the loss factor $\kappa \cong \omega_{\tau} / 2\left(\tilde{\omega}_{\mathrm{p}}-\omega\right)$ decreases rapidly with frequency $\omega$ decreasing below the renormalized plasma frequency $\tilde{\omega}_{\mathrm{p}}$. For the contrast $\left|\varepsilon_{\mathrm{m}}^{\prime}\right| / \varepsilon_{\mathrm{d}} \simeq 1$, the correlation function $G(r)$ can be estimated by Eq. (4.14). Substituting Eq. (4.14) in Eq. (4.12) and integrating up to $\xi_{\mathrm{e}} \sim a \kappa^{-1 / d}$ [see Eq. (2.24)], the following estimate is obtained:

$$
\begin{aligned}
S_{\mathrm{tot}} & \sim \frac{(k a)^{4}}{3}|T|^{2}\left|\varepsilon_{\mathrm{e}}\right|^{2} \kappa^{-1} \xi_{\mathrm{e}}^{1-\eta} \sim \frac{(k a)^{4}}{3}|T|^{2}\left|\varepsilon_{\mathrm{e}}\right|^{2} \kappa^{-1-(1-\eta) / \mathrm{d}} \\
& \sim \frac{(k a)^{4}}{3}|T|^{2} \varepsilon_{\mathrm{d}} \varepsilon_{\mathrm{b}}\left(\frac{\tilde{\omega}_{\mathrm{p}}}{\omega_{\tau}}\right)^{1+(1-\eta) / d}\left(1-\frac{\omega}{\tilde{\omega}_{\mathrm{p}}}\right)^{2+(1-\eta) / \mathrm{d}}, \quad \omega<\tilde{\omega}_{\mathrm{p}},
\end{aligned}
$$

where the Dykhne's formula is used for the effective dielectric constant at the percolation threshold: $\varepsilon_{\mathrm{e}}\left(p=p_{c}\right)=\sqrt{\varepsilon_{\mathrm{d}} \varepsilon_{\mathrm{m}}}$ for 2D percolation systems [100]. Eq. (4.15) holds for frequencies below $\tilde{\omega}_{\mathrm{p}}$.

Consider now the other limit $\omega \ll \tilde{\omega}_{\mathrm{p}}$, assuming again that $\omega \gg \omega_{\tau}$. Then the dielectric constant for a Drude metal can be approximated as $\varepsilon_{\mathrm{m}} \approx\left(\omega_{\mathrm{p}} / \omega\right)^{2}\left(-1+\mathrm{i} \omega_{\tau} / \omega\right)$ [see Eq. (2.1)] that gives 
$\left|\varepsilon_{\mathrm{m}}^{\prime}\right| / \varepsilon_{\mathrm{d}} \cong\left(\omega_{\mathrm{p}} / \omega\right)^{2} / \varepsilon_{\mathrm{d}} \gg 1$ and $\kappa=\varepsilon_{\mathrm{m}}^{\prime} /\left|\varepsilon_{\mathrm{m}}^{\prime}\right| \cong \omega / \omega_{\tau} \ll 1$. To estimate the behavior of the correlation function $G(r)$ the usual procedure of dividing the system into squares of size $l$ and considering each square as a new element is applied, as it has been discussed in Section 2.3. The square size $l$ is set equal to $l_{\mathrm{r}}$ given by Eq. (2.34). Then the correlation function $G^{\star}$ in the renormalized system has the form of Eq. (4.14), while in the original system it has the form $G(r) \cong\left(l_{\mathrm{r}} / a\right)^{1+\eta} G^{\star}(r)$ for $r \gg l_{\mathrm{r}}$. The function $G(r)$ follows the usual behavior of the current-current correlation function in a percolation system $G(r) \propto r^{-t / v}$ [107], for the distance $r \ll l_{\mathrm{r}}$. By matching these asymptotic expressions at $r=l_{\mathrm{r}}$, the following anzats for the correlation function is obtained:

$$
\begin{aligned}
& G(r) \sim M_{2}^{\star}\left(\frac{l_{\mathrm{r}}}{r}\right)^{t / v} \sim \kappa^{-1}\left(\frac{l_{\mathrm{r}}}{r}\right)^{t / v}, \quad a \ll r<l_{\mathrm{r}}, \\
& G(r) \sim M_{2}^{\star}\left(\frac{l_{\mathrm{r}}}{r}\right)^{1+\eta} \sim \kappa^{-1}\left(\frac{l_{\mathrm{r}}}{r}\right)^{1+\eta}, \quad l_{\mathrm{r}}<r<\xi_{\mathrm{e}},
\end{aligned}
$$

where $l_{\mathrm{r}}$ and $\xi_{\mathrm{e}}$ are given by Eqs. (2.34) and (2.40), respectively. The correlation function calculated for the loss parameter $\kappa=10^{-3}$ and contrast $\left|\varepsilon_{\mathrm{m}}^{\prime}\right| / \varepsilon_{\mathrm{d}}=10^{2}$, which corresponds to $l_{\mathrm{r}}=10 a$, is shown in Fig. 12. It is in reasonable agreement with the scaling of Eq. (4.16).

The scaling given by Eq. (4.16) allows to estimate the second moment of the local electric current $M_{j} \equiv\left\langle|\boldsymbol{j}(\boldsymbol{r})|^{2}\right\rangle$ at the percolation threshold $p_{c}$. The moment $M_{j}$ is expressed from Eq. (4.8) in terms of the current-current correlation function $G(r)$ as $M_{j}=\left|E_{0}\right|^{2}\left|\sigma_{\mathrm{e}}\right|^{2} G(0)=(\omega / 4 \pi)^{2}\left|E_{0}\right|^{2}\left|\varepsilon_{\mathrm{e}}\right|^{2} G(0)$, where $E_{0}$ is the external field, $\sigma_{\mathrm{e}}$ and $\varepsilon_{\mathrm{e}}$ are the effective conductivity and the dielectric constant, respectively. At the percolation threshold the effective dielectric constant is estimated as $\varepsilon_{\mathrm{e}} \sim$ $\varepsilon_{\mathrm{d}}\left(\varepsilon_{\mathrm{m}} / \varepsilon_{\mathrm{d}}\right)^{s /(s+t)}[12,20]$. To estimate the correlation function $G$ for $r \sim a$ the Eq. (4.16), obtained for distances $r$ much larger than the grain size $a$, is extrapolated to $r \rightarrow a$. Such an extrapolation gives $G(0) \sim G(a) \sim M_{2}^{\star}\left(l_{\mathrm{r}} / a\right)^{t / v} \sim M_{2}^{\star} \mid\left(\varepsilon_{\mathrm{m}} \mid / \varepsilon_{\mathrm{d}}\right)^{t /(s+t)}$. Combining the above estimates for $\varepsilon_{\mathrm{e}}$ and $G(0)$ the following equation is obtained:

$$
M_{j} \sim(\omega / 4 \pi)^{2}\left|E_{0}\right|^{2} \varepsilon_{\mathrm{d}}^{2} M_{2}^{\star}\left(\frac{\left|\varepsilon_{\mathrm{m}}\right|}{\varepsilon_{\mathrm{d}}}\right)^{(2 s+t) /(s+t)} \sim(\omega / 4 \pi)^{2}\left|E_{0}\right|^{2} \varepsilon_{\mathrm{d}}\left|\varepsilon_{\mathrm{m}}\right| M_{2},
$$

where in transition to the second estimate Eqs. (2.22) and (2.43) are used for the local field moments $M_{2}^{\star} \equiv M_{2,0}^{\star}$ and $M_{2} \equiv M_{2,0}$ correspondingly. This equation holds for arbitrary spatial dimension. In two dimensional case Eq. (4.17) coincides with exact Eq. (7.138) (see Section 7.3.4), which is held for self-dual composites at the percolation threshold.

Now we return to the consideration of the light scattering from semicontinuous metal films. Note that the frequency $\omega \ll \omega_{\mathrm{p}}$ is considered, when metal dielectric constant for a Drude metal [see Eq. (2.1)] can be approximated as $\varepsilon_{\mathrm{m}} \simeq-\left(\omega_{\mathrm{p}} / \omega\right)^{2}\left(1-\mathrm{i} \omega_{\tau} / \omega\right)$. By substituting the correlation function $G(r)$ from Eq. (4.16) in Eq. (4.12) and taking into account that at the percolation threshold $\left|\varepsilon_{\mathrm{e}}\right|^{2} \simeq \varepsilon_{\mathrm{d}}\left|\varepsilon_{\mathrm{m}}\right| \simeq \varepsilon_{\mathrm{d}}\left(\omega_{\mathrm{p}} / \omega\right)^{2}$, the following result is obtained:

$$
\begin{aligned}
S_{\mathrm{tot}} & \sim \frac{(k a)^{4}}{3}|T|^{2}\left|\varepsilon_{\mathrm{e}}\right|^{2} \kappa^{-1} l_{\mathrm{r}}^{1+\eta} \xi_{\mathrm{e}}^{1-\eta} \\
& \sim \frac{(k a)^{4}}{3}|T|^{2} \kappa^{-1-(1-\eta) / 2}\left|\varepsilon_{\mathrm{m}}\right|^{2} \sim 0.1\left(\frac{\omega_{\mathrm{p}} a}{c}\right)^{4}\left(\frac{\omega}{\omega_{\tau}}\right)^{1+(1-\eta) / 2},
\end{aligned}
$$


where the relation between the $2 \mathrm{D}$ percolation exponents $t \approx s \approx v \approx 4 / 3[12,20]$ is used as well as the experimental result $|T|^{2} \simeq 0.25$, which holds for $p=p_{c}$ and $\omega_{\tau} \ll \omega \ll \omega_{\mathrm{p}}$ [85]. Thus, the scattering first increases as $\omega^{1+(1-\eta) / 2}$ with increasing the frequency according to Eq. (4.18) and then vanishes as $\left(\tilde{\omega}_{\mathrm{p}}-\omega\right)^{2+(1-\eta) / 2}$ when $\omega$ approaches $\tilde{\omega}_{\mathrm{p}}$ [see Eq. (4.15)] in accordance with the numerical results shown in Fig. 11.

The enhancement of the scattering due to the field fluctuations can be estimated from Eqs. (4.13) and (4.16) as $g \sim|T|^{2} \varepsilon_{\mathrm{d}}\left|\varepsilon_{\mathrm{m}}\right| l_{\mathrm{r}}^{2} \kappa^{-1-(1-\eta) / 2} / 4$, which gives for a Drude metal [see Eq. (2.1)] and $\omega \ll \omega_{\mathrm{p}}$ the following equation:

$$
g \sim \frac{|T|^{2}}{4}\left(\frac{\tilde{\omega}_{\mathrm{p}}}{\omega}\right)^{4}\left(\frac{\omega}{\omega_{\tau}}\right)^{1+(1-\eta) / 2} .
$$

Taking the value $|T|^{2}=1 / 4$ and $\varepsilon_{\mathrm{d}}=2.2$, which is typical for a glass, the enhancement of scattering shown in Fig. 13 is obtained. The enhancement $g$ becomes as large as $g \sim 5 \times 10^{4}$ at wavelength $\lambda=1.5 \mu \mathrm{m}$ and continues to increase towards the far infrared spectral range. At first glance this continuous increase of the scattering enhancement $g$ seems to contradict to the behavior of the scattering shown in Fig. 11. It can be explained by noticing that Fig. 11 shows the anomalous scattering itself, whereas Fig. 13 shows the enhancement of the anomalous scattering with respect to Rayleigh scattering, assuming that metallic grains are independent. Rayleigh scattering decreases as $\omega^{4}$ with decreasing frequency, whereas the anomalous scattering varies as $S \sim \omega^{1+(1-\eta) / 2} \approx \omega^{1.1}$. Then the enhancement increases as $g \sim \omega^{-2.9} \sim \lambda^{2.9}$ in the infrared part of the spectrum.

In the formalism above it was assumed that the wavelength $\lambda$ is much larger than the grain size $a$ and that $\lambda$ is much larger than the spatial scale $\xi_{\mathrm{e}}$ of the giant field fluctuations, $\lambda \gg \xi_{\mathrm{e}}$. Consequently, the calculated Rayleigh scattering contains a small portion of the incident light only. The size of metal grains in semicontinuous metal films is usually of the order of few nanometers but it can be increased significantly by using a proper method of preparation [108]. In such a way the situation can be achieved, when the field correlation length $\xi_{\mathrm{e}}$ in Eq. (2.40) becomes larger than the wavelength $\lambda$ of the incident wave. In this case, the scattering can be a dominating process in the light interaction with semicontinuous metal films. Then some kind of critical opalescence, which is typical for critical phenomena, can be observed for these films. The consideration presented above holds for the spatial scales $a<l<\lambda$; one can speculate that the scattering is still given by Eq. (4.6), but the exponential term cannot be set as one. Integration in Eq. (4.6) over all directions of the vector $\boldsymbol{r}_{1}-\boldsymbol{r}_{2}$ gives the factor $J_{0}(k r \sin \theta)$, which means that the scattering is

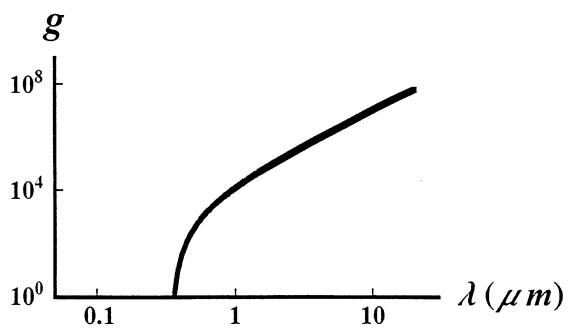

Fig. 13. Enhancement of Rayleigh scattering from semicontinuous gold films as a function of the wavelength; $p=p_{c}$. 
proportional to $S \propto \int_{0}^{\infty} G(r) J_{0}(k r \sin \theta) r \mathrm{~d} r$, where $\theta$ is the angle between the normal to the film plane and the direction of the scattered wave. Therefore, a thorough study of the conjectured "critical opalescence" from the films (e.g., the angular distribution of the scattered light) could give quantitative information on the spatial structure of the correlation function $G(r)$ and on the spatial structure of the giant field fluctuations in semicontinuous metal films.

After a possible analogy between the anomalous scattering and the critical opalescence is mentioned, now an important difference should be stressed between these phenomena. Indeed, the critical opalescence originates from the long-range fluctuations of some physical quantity, e.g., density fluctuations near the liquid-vapor critical point or fractal structure of metal clusters $[25,105]$, etc. In contrast to this, the spatial distribution of metal grains in semicontinuous metal films is random or correlated on the scales of a grain size $a \ll \lambda$. The anomalous light scattering originates from the long-range field fluctuations. Therefore, the incident electromagnetic wave plays a two-fold role: it first generates the giant fluctuations of local electric fields which in turn induce anomalous scattering. Considered anomalous scattering is, in a sense, a new kind of critical opalescence - the field opalescence.

\section{Raman scattering}

This section is concerned with surface-enhanced Raman scattering (SERS) - one of the most intriguing optical effects discovered in the past twenty years (see, for example, Refs. [24,109-112]). We present a theory of Raman scattering enhanced by strong field fluctuations of the local fields $[38,42]$. SERS from rough thin films is commonly associated with excitation of surface plasmon oscillations (see, e.g., [109,113]). Plasmon oscillations are typically considered in the two limiting cases: (1) oscillations in independent (noninteracting) roughness features of various shapes and (2) surface plasmon waves (polaritons) that laterally propagate along the metal surface (see $[109,113]$ and references therein). In reality, there are strong light-induced interactions between different features of a rough surface and, therefore, plasmon oscillations should be treated as collective surface excitations (localized surface plasmons) that depend strongly on the surface morphology as it has been explained in Section 2. Below, we present a theory that expresses the enhancement of Raman scattering in terms of the local field fluctuations.

\subsection{General formalism}

We consider optical properties of a semicontinuous metal film consisting of metal grains randomly distributed on a dielectric substrate. The gaps between metal grains are usually filled by dielectric material of the substrate. The local conductivity $\sigma(r)$ of the film takes either the "metallic" values, $\sigma(\boldsymbol{r})=\sigma_{\mathrm{m}}$, in the metal grains or the "dielectric" values, $\sigma(\boldsymbol{r})=-\mathrm{i} \omega \varepsilon_{\mathrm{d}} / 4 \pi$, outside the metal grains. Here $\omega$ is the frequency of the external field. It is supposed that the wavelength $\lambda$ is much larger than a typical scale of inhomogeneity in the composite including the grain size $a$, the gaps between the grains, percolation correlation length $\xi_{\mathrm{p}}$, and the local field correlation length $\xi_{\mathrm{e}}$ [see Eq. (2.40)]. In this case, the local field $\boldsymbol{E}(\boldsymbol{r})$ is given by Eq. (2.3). This equation is solved to find the fluctuating potentials $\phi(r)$ and the local fields $\boldsymbol{E}(\boldsymbol{r})$ induced in the film by the external field $\boldsymbol{E}_{0}(\boldsymbol{r})$. 
It is instructive to assume first that the external field $\boldsymbol{E}_{0}(\boldsymbol{r})$ is pin-like, $\boldsymbol{E}_{0}(\boldsymbol{r})=\boldsymbol{E}_{1} \delta\left(\boldsymbol{r}-\boldsymbol{r}_{1}\right)$, where the $\delta(\boldsymbol{r})$ is the Dirac delta function. The current density at arbitrary point $\boldsymbol{r}_{2}$ is given by the following linear relation:

$$
\boldsymbol{j}\left(\boldsymbol{r}_{2}\right)=\hat{\Sigma}\left(\boldsymbol{r}_{2}, \boldsymbol{r}_{1}\right) \boldsymbol{E}_{1}
$$

defining the nonlocal conductivity matrix $\hat{\Sigma}\left(\boldsymbol{r}_{2}, \boldsymbol{r}_{1}\right)$. This matrix represents the system's response at point $\boldsymbol{r}_{2}$ to the field source located at point $\boldsymbol{r}_{1}$. If an inhomogeneous external field $\boldsymbol{E}_{0}(\boldsymbol{r})$ is applied to the system the local current at point $\boldsymbol{r}_{2}$ is equal to

$$
\boldsymbol{j}\left(\boldsymbol{r}_{2}\right)=\int \widehat{\Sigma}\left(\boldsymbol{r}_{2}, \boldsymbol{r}_{1}\right) \boldsymbol{E}_{0}\left(\boldsymbol{r}_{1}\right) \mathrm{d} \boldsymbol{r}_{1},
$$

where the integration is over the total area of the system.

The nonlocal conductivity $\hat{\Sigma}$ can be expressed in terms of the Green function $G$ of Eq. (2.3):

$$
\nabla \cdot\left\{\sigma\left(\boldsymbol{r}_{2}\right)\left[\nabla G\left(\boldsymbol{r}_{2}, \boldsymbol{r}_{1}\right)\right]\right\}=\delta\left(\boldsymbol{r}_{2}-\boldsymbol{r}_{1}\right),
$$

where the differentiation with respect to the coordinate $\boldsymbol{r}_{2}$ is assumed. Comparing Eqs. (2.3) and (5.3) and using the definition of the nonlocal conductivity given in Eq. (5.1), the following equation is obtained

$$
\Sigma_{\alpha \beta}\left(\boldsymbol{r}_{2}, \boldsymbol{r}_{1}\right)=\sigma\left(\boldsymbol{r}_{2}\right) \sigma\left(\boldsymbol{r}_{1}\right) \frac{\partial^{2} G\left(\boldsymbol{r}_{2}, \boldsymbol{r}_{1}\right)}{\partial r_{2, \alpha} \partial r_{1, \beta}},
$$

where the Greek indices take values $x$ and $y$. As follows from the symmetry of Eq. (5.3), the Green function is symmetric with respect to the interchange of its arguments: $G\left(\boldsymbol{r}_{1}, \boldsymbol{r}_{2}\right)=G\left(\boldsymbol{r}_{2}, \boldsymbol{r}_{1}\right)$. Then, Eq. (5.4) implies that the nonlocal conductivity is also symmetric:

$$
\Sigma_{\alpha \beta}\left(\boldsymbol{r}_{1}, \boldsymbol{r}_{2}\right)=\Sigma_{\beta \alpha}\left(\boldsymbol{r}_{2}, \boldsymbol{r}_{1}\right) .
$$

The introduction of the nonlocal conductivity, $\hat{\Sigma}$, considerably simplifies further calculations of the local field distributions. The symmetry of $\hat{\Sigma}$ given by Eq. (5.5) is also important for the following analysis.

Since the wavelength of the incident em wave is much larger than all spatial scales in a semicontinuous metal film, the external field $\boldsymbol{E}_{0}$ is constant in the film plane. The local fields $\boldsymbol{E}\left(\boldsymbol{r}_{2}\right)$ induced by the external field $\boldsymbol{E}_{0}$ can be obtained by using Eq. (5.2) for the nonlocal conductivity $\hat{\Sigma}$ as follows:

$$
\boldsymbol{E}\left(\boldsymbol{r}_{2}\right)=\frac{1}{\sigma\left(\boldsymbol{r}_{2}\right)} \int \hat{\Sigma}\left(\boldsymbol{r}_{2}, \boldsymbol{r}_{1}\right) \boldsymbol{E}_{0} \mathrm{~d} \boldsymbol{r}_{1} .
$$

The local fields $\boldsymbol{E}\left(\boldsymbol{r}_{2}\right)$ excite Raman-active molecules that are assumed to be uniformly distributed in the composite. The Raman-active molecules, in turn, generate the Stokes fields $\boldsymbol{E}_{\mathrm{s}}\left(\boldsymbol{r}_{2}\right)=$ $\alpha_{\mathrm{s}}\left(\boldsymbol{r}_{2}\right) \boldsymbol{E}\left(\boldsymbol{r}_{2}\right)$, oscillating at the shifted frequency $\omega_{\mathrm{s}}\left[\alpha_{\mathrm{s}}\left(\boldsymbol{r}_{2}\right)\right.$ is the ratio for the Raman and linear polarizabilities of the Raman-active molecule at the point $\left.\boldsymbol{r}_{2}\right]$. The Stokes fields $\boldsymbol{E}_{\mathrm{s}}\left(\boldsymbol{r}_{2}\right)$ induce in the composite currents $\boldsymbol{j}_{\mathrm{s}}\left(\boldsymbol{r}_{3}\right)$ that are given by an equation similar to Eq. (5.6):

$$
\boldsymbol{j}_{\mathrm{s}}\left(\boldsymbol{r}_{3}\right)=\int \hat{\Sigma}\left(\boldsymbol{r}_{3}, \boldsymbol{r}_{2}\right) \boldsymbol{E}_{\mathrm{s}}\left(\boldsymbol{r}_{2}\right) \mathrm{d} \boldsymbol{r}_{2} .
$$


Since the Stokes-shifted frequency $\omega_{\mathrm{s}}$ is typically very close to the frequency of the external field $\left|\omega-\omega_{\mathrm{s}}\right| / \omega \ll 1$ the nonlocal conductivities $\hat{\Sigma}$, appearing in Eqs. (5.6) and (5.7), are considered to be the same.

The intensity of the electromagnetic wave $I$ scattered from any inhomogeneous system is proportional to the current fluctuations inside the system, as it has been discussed in all details in Section 4.1, namely

$$
I \propto\left\langle\left|\int[j(r)-\langle j\rangle] \mathrm{d} \boldsymbol{r}\right|^{2}\right\rangle,
$$

where the integration is over the entire system and the angular brackets $\langle\ldots\rangle$ denote the ensemble average. For Raman scattering, the mean includes averaging over the fluctuating phases of the incoherent Stokes fields generated by different Raman-active molecules. Therefore, the averaged current densities oscillating at $\omega_{\mathrm{s}}$ is zero, $\left\langle\boldsymbol{j}_{\mathrm{s}}\right\rangle=0$. Then, the intensity of Raman scattering $I_{\mathrm{s}}$ from a semicontinuous metal film acquires the following form:

$$
\begin{aligned}
I_{\mathrm{s}} & \propto\left\langle|j(\boldsymbol{r}) \mathrm{d} \boldsymbol{r}|^{2}\right\rangle \\
& =\int\left\langle\sum_{\alpha \beta}\left(\boldsymbol{r}_{3}, \boldsymbol{r}_{2}\right) \alpha_{\mathrm{s}}\left(\boldsymbol{r}_{2}\right) E_{\beta}\left(\boldsymbol{r}_{2}\right) \sum_{\alpha \gamma}^{*}\left(\boldsymbol{r}_{5}, \boldsymbol{r}_{4}\right) \alpha_{\mathrm{s}}^{*}\left(\boldsymbol{r}_{4}\right) E_{\gamma}^{*}\left(\boldsymbol{r}_{4}\right)\right\rangle \mathrm{d} \boldsymbol{r}_{2} \mathrm{~d} \boldsymbol{r}_{3} \mathrm{~d} \boldsymbol{r}_{4} \mathrm{~d} \boldsymbol{r}_{5},
\end{aligned}
$$

where the summation over repeating Greek indices is implied. All the integrations in Eq. (5.9) are over the entire film plane. Eq. (5.9) is averaged over the fluctuating phases of the Raman polarizabilities $\alpha_{\mathrm{s}}$. Since the Raman field sources are incoherent, this average results in

$$
\left\langle\alpha_{\mathrm{s}}\left(\boldsymbol{r}_{2}\right) \alpha_{\mathrm{s}}^{*}\left(\boldsymbol{r}_{4}\right)\right\rangle=\left|\alpha_{\mathrm{s}}\right|^{2} \delta\left(\boldsymbol{r}_{2}-\boldsymbol{r}_{4}\right)
$$

and Eq. (5.9) takes the following form:

$$
I_{\mathrm{s}} \propto \int\left\langle\sum_{\alpha \beta}\left(\boldsymbol{r}_{3}, \boldsymbol{r}_{2}\right) \sum_{\mu \gamma}^{*}\left(\boldsymbol{r}_{5}, \boldsymbol{r}_{2}\right) \delta_{\alpha \mu}\left|\alpha_{\mathrm{s}}\right|^{2} E_{\beta}\left(\boldsymbol{r}_{2}\right) E_{\gamma}^{*}\left(\boldsymbol{r}_{2}\right)\right\rangle \mathrm{d} \boldsymbol{r}_{2} \mathrm{~d} \boldsymbol{r}_{3} \mathrm{~d} \boldsymbol{r}_{5},
$$

where the Kronecker symbol $\delta_{\alpha \mu}$ is introduced to simplify further considerations. Since a semicontinuous film is macroscopically homogeneous, Raman scattering is independent of the orientation of the external field $\boldsymbol{E}_{0}$; therefore, Eq. (5.11) can be averaged over the orientations of the $\boldsymbol{E}_{0}$ without changing the result. The averaging of the products $E_{\beta}\left(\boldsymbol{r}_{2}\right) E_{\gamma}^{*}\left(\boldsymbol{r}_{2}\right)$ and $E_{0, \alpha} E_{0, \mu}^{*}$ results in the following expressions:

$$
\begin{aligned}
& \left\langle E_{\beta}\left(r_{2}\right) E_{\gamma}^{*}\left(r_{2}\right)\right\rangle_{0}=\frac{1}{2}\left\langle\left|E\left(r_{2}\right)\right|^{2}\right\rangle_{0} \delta_{\beta \gamma} \\
& \delta_{\alpha \mu}=2 \frac{\left\langle E_{0, \alpha} E_{0, \mu}^{*}\right\rangle_{0}}{\left|E_{0}\right|^{2}}
\end{aligned}
$$

where the sign $\langle\ldots\rangle_{0}$ denotes the orientation averaging. Substituting Eqs. (5.12) and (5.13) in Eq. (5.11) and noting that the nonlocal conductivity $\hat{\Sigma}$ is independent of the field orientations, the following result is obtained for the intensity of the Raman signal:

$$
\left\langle I_{\mathrm{s}}\right\rangle \propto \int \Sigma_{\alpha \beta}\left(r_{3}, r_{2}\right) \sum_{\mu \beta}^{*}\left(r_{5}, r_{2}\right) \frac{\left\langle E_{0, \alpha} E_{0, \mu}^{*}\right\rangle_{0}}{\left|E_{0}\right|^{2}}\left|\alpha_{\mathrm{s}}\right|^{2}\left\langle\left|E\left(r_{2}\right)\right|^{2}\right\rangle_{0} \mathrm{~d} r_{2} \mathrm{~d} r_{3} \mathrm{~d} r_{5} .
$$


[For simplicity, the sign for ensemble averaging is omitted here.] Now the symmetry of the nonlocal conductivity, given by Eq. (5.5), is used to rewrite Eq. (5.14) as

$$
\left\langle I_{\mathrm{s}}\right\rangle \propto \int\left\langle\sum_{\beta \alpha}\left(\boldsymbol{r}_{2}, \boldsymbol{r}_{3}\right) E_{0, \alpha} \Sigma_{\beta \mu}^{*}\left(\boldsymbol{r}_{2}, \boldsymbol{r}_{5}\right) E_{0, \mu}^{*}\right\rangle_{0} \frac{\left|\alpha_{\mathrm{s}}\right|^{2}}{\left|E_{0}\right|^{2}}\left\langle\left|E\left(\boldsymbol{r}_{2}\right)\right|^{2}\right\rangle_{0} \mathrm{~d} \boldsymbol{r}_{2} \mathrm{~d} \boldsymbol{r}_{3} \mathrm{~d} \boldsymbol{r}_{5} .
$$

Integration over the coordinates $\boldsymbol{r}_{3}$ and $\boldsymbol{r}_{5}$ and implementation of Eq. (5.2) gives

$$
\left\langle I_{\mathrm{s}}\right\rangle \propto \frac{\left|\alpha_{\mathrm{s}}\right|^{2}}{\left|E_{0}\right|^{2}} \int\left|\sigma\left(\boldsymbol{r}_{2}\right)\right|^{2}\left\langle\left|E\left(\boldsymbol{r}_{2}\right)\right|^{2}\right\rangle_{0}\left\langle\left|E\left(\boldsymbol{r}_{2}\right)\right|^{2}\right\rangle_{0} \mathrm{~d} \boldsymbol{r}_{2} .
$$

It is easy to show that this equation can be rewritten for macroscopically isotropic system in the following form:

$$
\left\langle I_{\mathrm{s}}\right\rangle \propto \frac{\left|\alpha_{\mathrm{s}}\right|^{2}}{\left|E_{0}\right|^{2}} \int\left|\sigma\left(\boldsymbol{r}_{2}\right)\right|^{2}\left|E\left(\boldsymbol{r}_{2}\right)\right|^{4} \mathrm{~d} \boldsymbol{r}_{2} .
$$

If there were no metal grains on the film, the local fields would not fluctuate and one would obtain the following expression for Raman scattering:

$$
I_{\mathrm{s}}^{0} \propto \int\left|\sigma_{\mathrm{d}}\right|^{2}\left|\alpha_{\mathrm{s}}\right|^{2}\left|E_{0}\right|^{2} \mathrm{~d} \boldsymbol{r}_{2} .
$$

Therefore, the enhancement of Raman scattering $G_{\mathrm{RS}}$ due to presence of metal grains on a dielectric substrate is given by

$$
G_{\mathrm{RS}}=\frac{\left\langle I_{\mathrm{s}}\right\rangle}{I_{\mathrm{s}}^{0}}=\frac{\left\langle|\sigma(\boldsymbol{r})|^{2}|E(\boldsymbol{r})|^{4}\right\rangle}{\left|\sigma_{\mathrm{d}}\right|^{2}\left|E_{0}\right|^{4}}=\frac{\left\langle|\varepsilon(\boldsymbol{r})|^{2}|E(\boldsymbol{r})|^{4}\right\rangle}{\varepsilon_{\mathrm{d}}^{2}\left|E_{0}\right|^{4}} .
$$

Note that the derivation of Eq. (5.19) is essentially independent of the dimensionality and morphology of a system. Therefore, the enhancement of Raman scattering given by Eq. (5.19) holds for any inhomogeneous system provided the field fluctuations take place inside it. In particular, Eq. (5.19) gives the enhancement for Raman scattering from a rough metallic surface, provided the wavelength is much larger than the roughness spatial scales; it can be also used to calculate enhancements for Raman scattering in a three-dimensional percolation composites. The presented theory, which was developed in Refs. $[38,42]$, implies that the main sources for the Raman signal are the currents excited by Raman molecules in metal grains. This explains why a significant enhancement for Raman scattering is observed even for relatively flat metal surfaces $[109,113,114]$.

\subsection{Raman and hyper-Raman scattering in metal-dielectric composites}

It has been shown in Section 2 that in percolation composites the local electric field concentrates mainly in the dielectric gaps between metal clusters. Then the SERS enhancement given by Eq. (5.19) can be estimated as $G_{\mathrm{RS}} \sim M_{4,0}=\left\langle\left|E(\boldsymbol{r}) / E_{0}\right|^{4}\right\rangle$. Eq. (2.43) for the fourth moment gives

$$
G_{\mathrm{RS}} \sim \rho(p)\left[\xi_{\mathrm{A}}(p) / a\right]^{d-8}\left(\frac{\left|\varepsilon_{\mathrm{m}}\right|}{\varepsilon_{\mathrm{d}}}\right)^{(2 v+s) /(t+s)}\left(\frac{\left|\varepsilon_{\mathrm{m}}\right|}{\varepsilon_{\mathrm{m}}^{\prime \prime}}\right)^{3},
$$


where the dependence of the density of states $\rho(p)$ and localization length $\xi_{\mathrm{A}}(p)$ on the concentration $p$ of metal grains is indicated explicitly. Thus the obtained Raman enhancement $G_{\mathrm{RS}}$ depends strongly on localization length $\xi_{\mathrm{A}}$. When the states are delocalized, $\xi_{\mathrm{A}} \rightarrow \infty$ and $G_{\mathrm{RS}}$ vanishes very rapidly.

Now the frequency and concentration dependence of Raman scattering predicted by Eq. (5.20) is considered. For 2D metal-dielectric composites (semicontinuous metal films) the critical exponents $s \approx t \approx v \approx 4 / 3$. The Drude metal dielectric function (2.1) can be used for frequency $\omega \ll \omega_{\mathrm{p}}$, and Eq. (5.20) results in the enhancement $G_{\mathrm{RS}} \sim \rho(p)\left[a / \xi_{\mathrm{A}}(p)\right]^{6}\left(\omega_{\mathrm{p}} / \omega_{\tau}\right)^{3} / \varepsilon_{\mathrm{d}}^{3 / 2}$, which is independent of frequency.

For silver-on-glass percolation films, the Anderson localization length $\xi_{\mathrm{A}}$ is set $\xi_{\mathrm{A}} \approx 2 a$ (see Figs. 1-4), which gives the best fit for the moments in Fig. 5, and density of state $\rho\left(p_{c}\right) \sim 1$ [see discussion after Eq. (2.12)]. Thus the SERS $G_{\mathrm{RS}}$ achieves the values $G_{\mathrm{RS}} \sim 10^{6}$ in silver semicontinuous films at the percolation threshold. For 3D composite at $\omega \ll \omega_{\mathrm{p}}$, SERS decreases with decreasing frequency as $G_{\mathrm{RS}} \sim \rho(p)\left(\xi_{\mathrm{A}} / a\right)^{-5} \omega_{\mathrm{p}}^{2} \omega / \omega_{\tau}^{3} \sim 10^{6} \omega / \omega_{\mathrm{p}}$, where the estimates $\xi_{\mathrm{A}} \approx 2 a, \rho \sim 1$ are used and 3D critical exponents are approximated as $v \approx s \approx(t+s) / 3[12,20]$; the data $\omega_{\mathrm{p}}=9.1 \mathrm{eV}$ and $\omega_{\tau}=0.021 \mathrm{eV}$ for silver dielectric constant [104] are substituted.

The localization radius $\xi_{\mathrm{A}}$ of the eigenstates $\Psi_{n}$ with eigenvalues $\Lambda \approx 0$ decreases when concentration shifts from $p=p_{c}$ toward $p=0$ or $p=1$ since the eigenvalue $\Lambda=0$ shifts from the center of the $\Lambda$-distribution to its tails, where localization of the eigenstates is stronger [see discussion at Eq. (2.25) in Section 2]. Therefore, according to Eq. (5.20) Raman scattering has a minimum at the percolation threshold. As a result, the double maximum dependence $G_{\mathrm{RS}}(p)$ takes place as it was observed in experiments and numerical calculations [38,42], with one maximum below the percolation threshold $p_{c}$ and another above the $p_{c}$ as it is shown in Fig. 14.

Results of experimental studies of the SERS dependence on the metal filling factor $p$ compared to theoretical calculations are shown in Fig. 15 taken from [42]. One can see that there is good qualitative agreement between predictions of theory and experimental observations. In particular, in accordance with theory, there is a dip near the percolation threshold in the SERS dependence on $p$.

The intensity of the local Stokes sources $I_{\mathrm{RS}}(\boldsymbol{r}) \propto|E(\boldsymbol{r})|^{4}$ (provided the Stokes shift of frequency is small) follows the local field distribution. In the peaks (hot spots), Eq. (2.35) gives

$$
I_{\mathrm{RS}, \max } \propto|E(\boldsymbol{r})|^{4} \sim E_{0}^{4}\left(a / \xi_{\mathrm{A}}\right)^{8}\left(\frac{\left|\varepsilon_{\mathrm{m}}\right|}{\varepsilon_{\mathrm{d}}}\right)^{4 v /(t+s)}\left(\frac{\left|\varepsilon_{\mathrm{m}}\right|}{\varepsilon_{\mathrm{m}}^{\prime \prime}}\right)^{4} .
$$

For a semicontinuous Drude metal film at $p=p_{c}$ and $\omega \ll \omega_{\mathrm{p}}$, the peak intensity is estimated as $I_{\mathrm{RS}, \max } \propto|E(\boldsymbol{r})|^{4} / E_{0}^{4} \sim\left(\xi_{\mathrm{A}} / a\right)^{-8}\left(\omega_{\mathrm{p}} / \omega_{\tau}\right)^{4} \gg 1$. If the density of Raman-active molecules is small enough, then each peak of the local field can be due to Raman scattering from a single molecule. The distribution of the local Raman signal in silver semicontinuous film is shown in Fig. 16.

Consider now hyper-Raman scattering when $n$ photons of frequency $\omega$ are converted in one hyper-Stokes photon of frequency $\omega_{\mathrm{hRS}}=n \omega-\Omega$, where $\Omega$ is the Stokes frequency shift corresponding to the frequency of molecule oscillations (electronic or vibrational). Following the general approach, described in the beginning of this section (see Ref. [38]), the surface enhancement of 


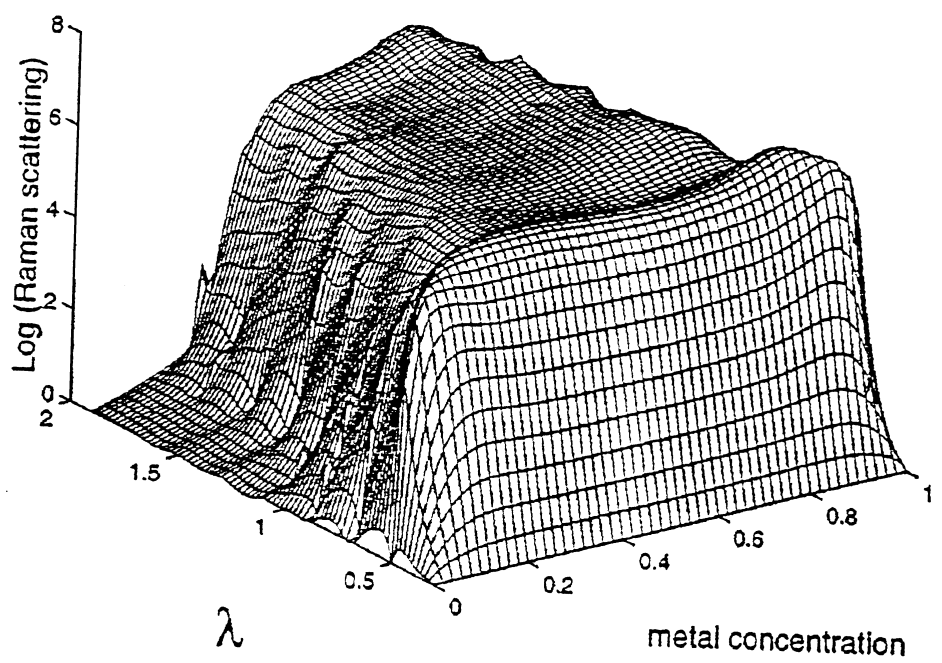

Fig. 14. The enhancement factor $G_{\mathrm{RS}}$ for Raman scattering from a silver semicontinuous film as a function of the metal concentration $p$ and the wavelength, $\lambda$.

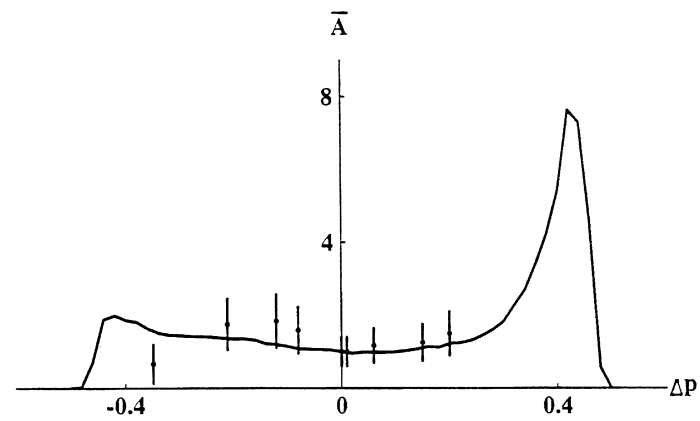

Fig. 15. Normalized SERS $\bar{A}=G_{\mathrm{RS}}(p) / G_{\mathrm{RS}}\left(p=p_{c}\right)$ as a function of the metal concentration, $\Delta p=p-p_{c}$, on a silver semicontinuous film. The solid curve represents theoretical calculations; the points are experimental data.

hyper-Raman scattering (SEHRS) $G_{\mathrm{hRS}}$ is equal to

$$
G_{\mathrm{hRS}}=\frac{\left\langle\left|\sigma_{\mathrm{hRS}}(\boldsymbol{r})\right|^{2}\left|\boldsymbol{E}_{\mathrm{hRS}}(\boldsymbol{r})\right|^{2}|E(\boldsymbol{r})|^{2 n}\right\rangle}{\left|\sigma_{\mathrm{d}}\right|^{2}\left|E_{0, \mathrm{hRS}}\right|^{2}\left|E_{0}\right|^{2 n}}=\frac{\left\langle\left|\varepsilon_{\mathrm{hRS}}(\boldsymbol{r})\right|^{2}\left|\boldsymbol{E}_{\mathrm{hRS}}(\boldsymbol{r})\right|^{2}|E(\boldsymbol{r})|^{2 n}\right\rangle}{\left|\varepsilon_{\mathrm{d}}\right|^{2}\left|E_{0, \mathrm{hRS}}\right|^{2}\left|E_{0}\right|^{2 n}}
$$

where $\boldsymbol{E}_{\mathrm{hRS}}(\boldsymbol{r})$ is the local field excited in the system by the uniform probe field $\boldsymbol{E}_{0, \mathrm{hRS}}$ oscillating with $\omega_{\mathrm{hRS}} ; \sigma_{\mathrm{hRS}}(\boldsymbol{r})$ and $\varepsilon_{\mathrm{hRS}}(\boldsymbol{r})$ are the local conductivity and dielectric constant at the frequency $\omega_{\mathrm{hRS}}$. At $n=1$ formula (5.22) describes the conventional SERS.

To estimate $G_{\mathrm{hRS}}$ it is taken into account that the spatial scales for the field maxima $l_{\mathrm{r}}$ [see Eq. (2.34)] at the fundamental frequency $\omega$ and hyper-Stokes frequency $\omega_{\mathrm{hRs}}$ are significantly different. Therefore the average in Eq. (5.19) might be decoupled and approximated as 


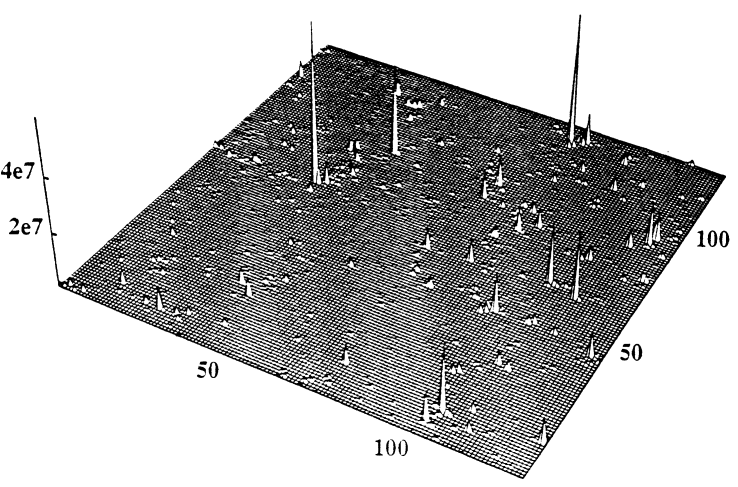

(a)

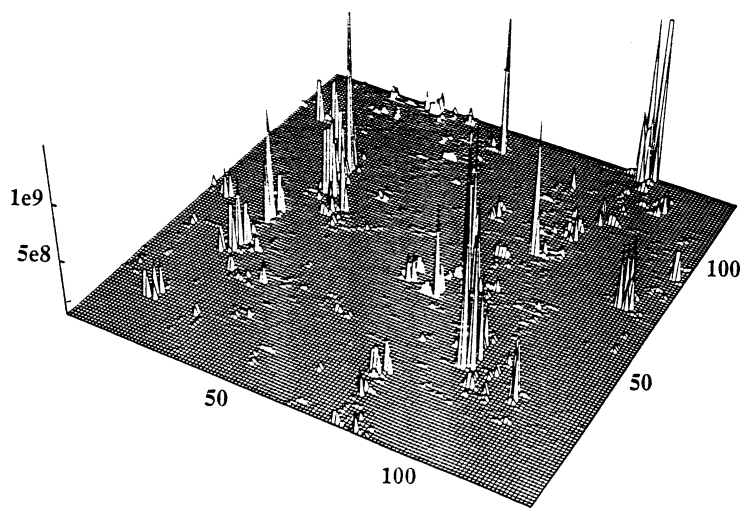

(c)

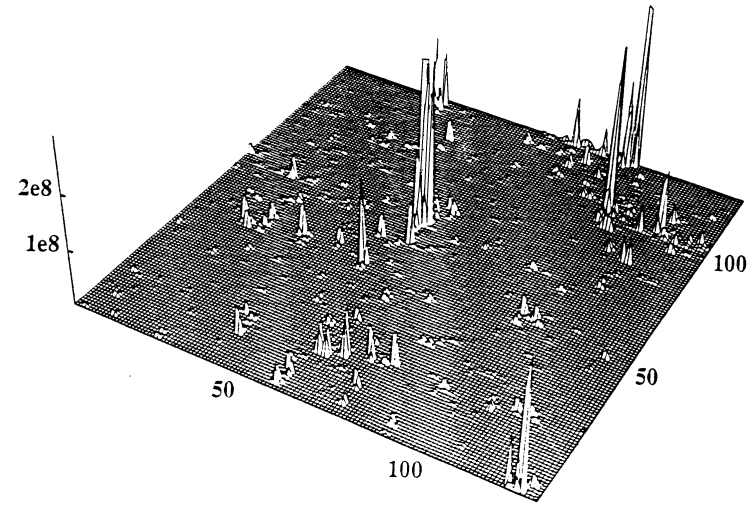

(b)

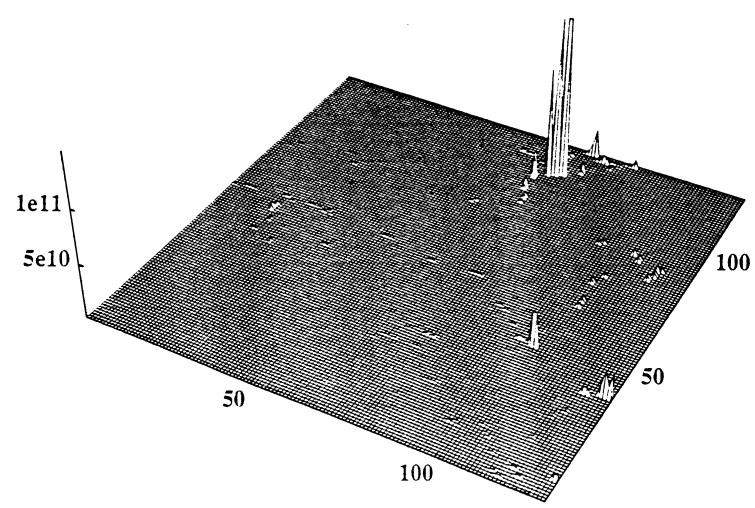

(d)

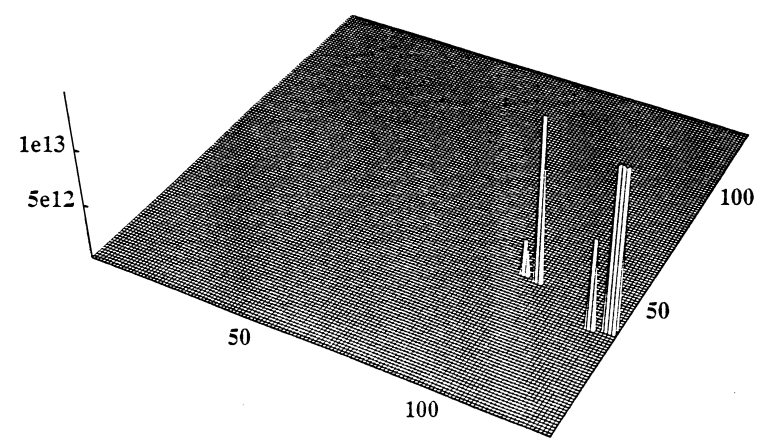

(e)

Fig. 16. Distribution of the local enhancement of Raman scattering on a silver semicontinuous film at the percolation threshold for different wavelengths: (a) $\lambda=0.36 \mu \mathrm{m}$, (b) $\lambda=0.5 \mu \mathrm{m}$, (c) $\lambda=1.5 \mu \mathrm{m}$, (d) $\lambda=10 \mu \mathrm{m}$, (e) $\lambda=20 \mu \mathrm{m}$. 
$\left\langle\left|\varepsilon_{\mathrm{hRS}}(\boldsymbol{r})\right|^{2}\left|\boldsymbol{E}_{\mathrm{hRS}}(\boldsymbol{r})\right|^{2}|E(\boldsymbol{r})|^{2 n}\right\rangle \sim\left\langle\left|\varepsilon_{\mathrm{hRS}}(\boldsymbol{r}) \boldsymbol{E}_{\mathrm{hRS}}(\boldsymbol{r})\right|^{2}\right\rangle\left\langle|E(\boldsymbol{r})|^{2 n}\right\rangle=\left\langle\left|\varepsilon_{\mathrm{hRS}}(\boldsymbol{r}) \boldsymbol{E}_{\mathrm{hRS}}(\boldsymbol{r})\right|^{2}\right\rangle M_{2 n}\left|E_{0}\right|^{2 n}, \quad$ where $M_{2 n}(\omega)$, given by Eq. (2.27), is the $2 n$th moment of the local field $E(r)$. It follows from Eq. (4.17) that the second moment of the current $\left\langle\left|\varepsilon_{\mathrm{hRS}}(\boldsymbol{r}) \boldsymbol{E}_{\mathrm{hRS}}(\boldsymbol{r})\right|^{2}\right\rangle$ is estimated as $\varepsilon_{\mathrm{d}}\left|\varepsilon_{\mathrm{m}}\left(\omega_{\mathrm{hRS}}\right)\right| M_{2}\left|E_{0, \mathrm{hRS}}\right|^{2}$, where the moment $M_{2}\left(\omega_{\mathrm{hRS}}\right)$ is the second moment of the field $\boldsymbol{E}_{\mathrm{hRS}}(\boldsymbol{r})$. Substitution of these results in Eq. (5.19) gives

$$
G_{\mathrm{hRS}} \sim \frac{\left|\varepsilon_{\mathrm{m}}\left(\omega_{\mathrm{hRS}}\right)\right|}{\varepsilon_{\mathrm{d}}} M_{2}\left(\omega_{\mathrm{hRS}}\right) M_{2 n}(\omega),
$$

where the moment $M_{2}$ is taken at frequency $\omega_{\mathrm{hRs}}$. Now the expressions for moments $M_{2}$ and $M_{2 n}$ given by Eq. (2.43) are substituted in the above equation and it is taken into account that for $p \simeq p_{c}$ the density of states in Eq. (2.43) is about unity $\rho \sim 1$. Thus the following formula for enhancement of hyper-Raman scattering is obtained:

$$
\begin{aligned}
G_{\mathrm{hRS}} \sim & \left(\xi_{\mathrm{A}} / a\right)^{2 d-4(1+n)}\left(\frac{\left|\varepsilon_{\mathrm{m}}\left(\omega_{\mathrm{hRS}}\right)\right|}{\varepsilon_{\mathrm{d}}}\right)^{(t+2 s) /(t+s)}\left(\frac{\left|\varepsilon_{\mathrm{m}}\left(\omega_{\mathrm{hRS}}\right)\right|}{\varepsilon_{\mathrm{m}}^{\prime \prime}\left(\omega_{\mathrm{hRS}}\right)}\right) \\
& \times\left(\frac{\left|\varepsilon_{\mathrm{m}}(\omega)\right|}{\varepsilon_{\mathrm{d}}}\right)^{(2 v(n-1)+s) /(t+s)}\left(\frac{\left|\varepsilon_{\mathrm{m}}(\omega)\right|}{\varepsilon_{\mathrm{m}}^{\prime \prime}(\omega)}\right)^{2 n-1},
\end{aligned}
$$

where $n \geq 2$. For a Drude metal and frequencies $\omega \ll \tilde{\omega}_{\mathrm{p}}, \omega_{\mathrm{hRS}} \ll \tilde{\omega}_{\mathrm{p}}$ the metal dielectric constant can be approximated as $\left|\varepsilon_{\mathrm{m}}\left(\omega_{\mathrm{hRS}}\right)\right| \sim\left|\varepsilon_{\mathrm{m}}(\omega)\right| \sim\left(\omega_{\mathrm{p}} / \omega\right)^{2}, \quad \varepsilon_{\mathrm{m}}^{\prime \prime}(\omega) /\left|\varepsilon_{\mathrm{m}}(\omega)\right| \sim \omega_{\tau} / \omega$ and Eq. (5.24) acquires the form

$$
G_{\mathrm{hRS}} \sim\left(\xi_{\mathrm{A}} / a\right)^{2 d-4(1+n)}\left(\frac{\omega_{\mathrm{p}}}{\omega}\right)^{2(2 v(n-1)+3 s+t) /(t+s)}\left(\frac{\omega}{\omega_{\tau}}\right)^{2 n},
$$

which holds in the vicinity to the percolation threshold. For 2D composites where the critical exponents are $t \approx s \approx v \approx 4 / 3$ Eq. (5.24) simplifies to

$$
G_{\mathrm{hRS}} \sim\left(a / \xi_{\mathrm{A}}\right)^{4 n}\left(\frac{\omega_{\mathrm{p}}}{\omega}\right)^{2(n+1)}\left(\frac{\omega}{\omega_{\tau}}\right)^{2 n} \sim\left(a / \xi_{\mathrm{A}}\right)^{4 n}\left(\frac{\omega_{\mathrm{p}}}{\omega}\right)^{2}\left(\frac{\omega_{\mathrm{p}}}{\omega_{\tau}}\right)^{2 n},
$$

which for $n=2$ in silver semicontinuous films is estimated as $G_{\mathrm{hRS}} \sim 10^{13}\left(a / \xi_{\mathrm{A}}\right)^{8} \lambda^{2}$, where the wavelength $\lambda$ is given in microns. As above for Raman scattering, the local enhancement in the hot spots can be larger than the average one by many orders of magnitude.

\section{Giant enhancements of optical nonlinearities in metal-dielectric composites}

In this section, we consider enhancements in percolation composites for different nonlinear optical processes nonlinearities, such as Kerr optical effect and generation of high harmonics.

\subsection{Kerr-type optical nonlinearities}

The Kerr-type nonlinearities are third-order optical nonlinearities that result in additional term in the electric displacement $\boldsymbol{D}$ of the form

$$
D_{\alpha}^{(3)}(\omega)=\varepsilon_{\alpha \beta \gamma \delta}^{(3)}(-\omega ; \omega, \omega,-\omega) E_{\beta} E_{\gamma} E_{\delta}^{*},
$$


where

$$
\varepsilon_{\alpha \beta \gamma \delta}^{(3)}(-\omega ; \omega, \omega,-\omega) .
$$

is the third-order nonlinear dielectric constant [81,82], and $\boldsymbol{E}$ is an electric field at frequency $\omega$; summation over repeated Greek indices is implied. The Kerr optical nonlinearity results in nonlinear corrections (proportional to the light intensity) for the refractive index and the absorption coefficient.

Below macroscopically homogeneous and isotropic composites are considered. Then the thirdorder terms in the average electric displacement has, in general, the following form:

$$
\left\langle\boldsymbol{D}^{(3)}(\boldsymbol{r})\right\rangle=\alpha\left|\boldsymbol{E}_{0}\right|^{2} \boldsymbol{E}_{0}+\beta E_{0}^{2} \boldsymbol{E}_{0}^{*},
$$

where $\boldsymbol{E}_{0}$ is the amplitude of the external (macroscopic) electric field at frequency $\omega, E_{0}^{2} \equiv\left(\boldsymbol{E}_{0} \cdot \boldsymbol{E}_{0}\right)$, $\alpha$ and $\beta$ are some constants [not to be confused with the tensor components in Eq. (6.1)]. Note that the second term in Eq. (6.3) for the nonlinear displacement of an isotropic film can result in change of the polarization of the incident light [81]. Eq. (6.3) simplifies for the case of linear and circular polarization of the incident light [81]. For the linear polarization the complex vector $\boldsymbol{E}_{0}$ reduces to a real vector. Then the expressions $\left|\boldsymbol{E}_{0}\right|^{2} \boldsymbol{E}_{0}$ and $E_{0}^{2} \boldsymbol{E}_{0}$ in Eq. (6.3) become the same and the equation can be rewritten as

$$
\left\langle\boldsymbol{D}^{(3)}(\boldsymbol{r})\right\rangle=\varepsilon_{\mathrm{e}}^{(3)}\left|E_{0}\right|^{2} \boldsymbol{E}_{0},
$$

where the nonlinear dielectric constant $\varepsilon_{\mathrm{e}}^{(3)}$ is a scalar now. For the sake of simplicity, we consider below the linearly polarized incident wave. Eq. (6.4), being rewritten in terms of the nonlinear average current $\left\langle\boldsymbol{j}^{(3)}(\boldsymbol{r})\right\rangle$ and the effective Kerr conductivity $\sigma_{\mathrm{e}}^{(3)}=-\mathrm{i} \omega \varepsilon_{\mathrm{e}}^{(3)} / 4 \pi$, takes the following form:

$$
\left\langle\boldsymbol{j}^{(3)}(\boldsymbol{r})\right\rangle=\sigma_{\mathrm{e}}^{(3)}\left|E_{0}\right|^{2} \boldsymbol{E}_{0} .
$$

This form of the Kerr nonlinearity is used in the discussion below.

First the case when the nonlinearities in metal grains $\sigma_{\mathrm{m}}^{(3)}$ and dielectric $\sigma_{\mathrm{d}}^{(3)}$ are close to each other $\sigma_{\mathrm{m}}^{(3)} \approx \sigma_{\mathrm{d}}^{(3)} \approx \sigma^{(3)}$ is considered. For example it can be due to molecules uniformly covering a semicontinuous film. [The composites, where $\left|\sigma_{\mathrm{m}}^{(3)}\right| \gg\left|\sigma_{\mathrm{d}}^{(3)}\right|$, are discussed below at Eq. (6.21).] When $\sigma_{\mathrm{m}}^{(3)} \approx \sigma_{\mathrm{d}}^{(3)} \approx \sigma^{(3)}$ the current in the composite is given by

$$
\boldsymbol{j}(\boldsymbol{r})=\sigma^{(1)}(\boldsymbol{r}) \boldsymbol{E}^{\prime}(\boldsymbol{r})+\sigma^{(3)}\left|\boldsymbol{E}^{\prime}(\boldsymbol{r})\right|^{2} \boldsymbol{E}^{\prime}(\boldsymbol{r}),
$$

where $\boldsymbol{E}^{\prime}(\boldsymbol{r})$ is the local fluctuating field. When current is given by Eq. (6.6) the current conservation law given by Eq. (2.3) takes the following form:

$$
\nabla \cdot\left(\sigma^{(1)}(\boldsymbol{r})\left[-\nabla \phi(\boldsymbol{r})+\boldsymbol{E}_{0}+\frac{\sigma^{(3)}}{\sigma^{(1)}(\boldsymbol{r})} \boldsymbol{E}^{\prime}(\boldsymbol{r})\left|\boldsymbol{E}^{\prime}(\boldsymbol{r})\right|^{2}\right]\right)=0,
$$

where $\boldsymbol{E}_{0}$ is the applied electric field, and $-\nabla \phi(\boldsymbol{r})+\boldsymbol{E}_{0}=\boldsymbol{E}^{\prime}(\boldsymbol{r})$ is the local field. It is convenient to consider the last two terms in the square brackets in Eq. (6.7) as a renormalized external field

$$
\boldsymbol{E}_{\mathrm{e}}(\boldsymbol{r})=\boldsymbol{E}_{0}+\boldsymbol{E}_{\mathrm{f}}(\boldsymbol{r})=\boldsymbol{E}_{0}+\frac{\sigma^{(3)}}{\sigma^{(1)}(\boldsymbol{r})} \boldsymbol{E}^{\prime}(\boldsymbol{r})\left|\boldsymbol{E}^{\prime}(\boldsymbol{r})\right|^{2},
$$


where the field

$$
\boldsymbol{E}_{\mathrm{f}}(\boldsymbol{r})=\frac{\sigma^{(3)}}{\sigma^{(1)}(\boldsymbol{r})} \boldsymbol{E}^{\prime}(\boldsymbol{r})\left|\boldsymbol{E}^{\prime}(\boldsymbol{r})\right|^{2}
$$

may change over the film but its averaged value $\left\langle\boldsymbol{E}_{\mathrm{f}}(\boldsymbol{r})\right\rangle$ is collinear to $\boldsymbol{E}_{0}$. Then the average current density $\langle\boldsymbol{j}(\boldsymbol{r})\rangle$ is also collinear to $\boldsymbol{E}_{0}$ in macroscopically isotropic films considered here. Therefore, the average current can be written as

$$
\langle\boldsymbol{j}\rangle=\frac{\boldsymbol{E}_{0}}{E_{0}^{2}}\left(\boldsymbol{E}_{0} \cdot\langle\boldsymbol{j}\rangle\right)=\frac{\boldsymbol{E}_{0}}{E_{0}^{2}} \frac{1}{A} \int \boldsymbol{E}_{0} \cdot \boldsymbol{j}(\boldsymbol{r}) \mathrm{d} \boldsymbol{r},
$$

where $A$ is the total area of the film, the integration is over the film area and $E_{0}^{2} \equiv\left(\boldsymbol{E}_{0} \cdot \boldsymbol{E}_{0}\right)$. Expressing the current $j(r)$ in Eq. (6.10) in terms of the nonlocal conductivity matrix defined by Eq. (5.1) gives

$$
\langle\boldsymbol{j}\rangle=\frac{\boldsymbol{E}_{0}}{E_{0}^{2}} \frac{1}{A} \int\left[\boldsymbol{E}_{0} \hat{\Sigma}\left(\boldsymbol{r}, \boldsymbol{r}_{1}\right) \boldsymbol{E}_{\mathrm{e}}\left(\boldsymbol{r}_{1}\right)\right] \mathrm{d} \boldsymbol{r} \mathrm{d} \boldsymbol{r}_{1},
$$

where the integrations are over the entire film. Now this equation is integrated over the coordinates $\boldsymbol{r}$ and the symmetry of the matrix of nonlocal conductivity given by Eq. (5.5) is used. This results in the expression

$$
\langle\boldsymbol{j}\rangle=\frac{\boldsymbol{E}_{0}}{E_{0}^{2}} \frac{1}{A} \int\left(\boldsymbol{j}_{0}(\boldsymbol{r}) \cdot \boldsymbol{E}_{\mathrm{e}}(\boldsymbol{r})\right) \mathrm{d} \boldsymbol{r},
$$

where $\boldsymbol{j}_{0}(\boldsymbol{r})$ is the current induced at the coordinate $\boldsymbol{r}$ by the constant external field $\boldsymbol{E}_{0}$. Now we can substitute in Eq. (6.12) the renormalized external field $\boldsymbol{E}_{\mathrm{e}}(\boldsymbol{r})$ from Eq. (6.8) and integrate over the coordinate which gives for the average current

$$
\langle\boldsymbol{j}\rangle=E_{0}\left[\sigma_{\mathrm{e}}^{(1)}+\frac{\left\langle\sigma^{(3)}\left(\boldsymbol{E}(\boldsymbol{r}) \cdot \boldsymbol{E}^{\prime}(\boldsymbol{r})\right)\left|\boldsymbol{E}^{\prime}(\boldsymbol{r})\right|^{2}\right\rangle}{E_{0}^{2}}\right],
$$

where $\sigma_{\mathrm{e}}^{(1)}$ and $\boldsymbol{E}(\boldsymbol{r})$ are the effective conductivity and local fluctuating field, respectively, obtained in the linear approximation, i.e., for $\sigma^{(3)} \equiv 0$. Comparison of Eqs. (6.13) and (6.5), allows to find the equation for the effective Kerr conductivity

$$
\sigma_{\mathrm{e}}^{(3)}=\frac{\left\langle\sigma^{(3)}\left(\boldsymbol{E}(\boldsymbol{r}) \cdot \boldsymbol{E}^{\prime}(\boldsymbol{r})\right)\left|\boldsymbol{E}^{\prime}(\boldsymbol{r})\right|^{2}\right\rangle}{E_{0}^{2}\left|E_{0}\right|^{2}} .
$$

Let us stress out that this result does not depend on the "weakness" of the nonlinearity, it holds even for strong nonlinear case $\left|\sigma^{(3)} E^{2}\right| \gg \sigma_{\mathrm{e}}^{(1)}$. For the case of weak nonlinearities the local field $\boldsymbol{E}^{\prime}(\boldsymbol{r})$ in Eq. (6.14) can be replaced by the linear local field $\boldsymbol{E}(\boldsymbol{r})$ resulting in the following equation for the Kerr conductivity

$$
\sigma_{\mathrm{e}}^{(3)}=\frac{\left\langle\sigma^{(3)} E^{2}(\boldsymbol{r})|\boldsymbol{E}(\boldsymbol{r})|^{2}\right\rangle}{E_{0}^{2}\left|E_{0}\right|^{2}},
$$

which gives the effective nonlinear conductivity in terms of the linear local field. 
In the absence of metal grains the effective nonlinear Kerr conductivity $\sigma_{\mathrm{e}}^{(3)}$ coincides with the Kerr conductivity $\sigma^{(3)}$ of the dielectric. Therefore the enhancement of the Kerr nonlinearity $G_{\mathrm{K}}$ is given by the following equation:

$$
G_{\mathrm{K}}=\frac{\left\langle E^{2}(\boldsymbol{r})|\boldsymbol{E}(\boldsymbol{r})|^{2}\right\rangle}{E_{0}^{2}\left|E_{0}\right|^{2}}=M_{2,2},
$$

where $M_{2,2}$ is the fourth moment of the local field [see Eq. (2.26)]. Therefore, the enhancement of the Kerr nonlinearity $G_{\mathrm{K}}$ is expressed in terms of the local field $\boldsymbol{E}(\boldsymbol{r})$ found in the linear approximation.

Above it has been assumed that the nonlinear Kerr conductivity $\sigma^{(3)}$ is the same in metal and dielectric. When $\sigma_{\mathrm{m}}^{(3)} \neq \sigma_{\mathrm{d}}^{(3)}$ the above derivations can be repeated and the following result emerges for the effective Kerr conductivity

$$
\sigma_{\mathrm{e}}^{(3)}=p \sigma_{\mathrm{m}}^{(3)} \frac{\left\langle E^{2}(\boldsymbol{r})|\boldsymbol{E}(\boldsymbol{r})|^{2}\right\rangle_{\mathrm{m}}}{E_{0}^{2}\left|E_{0}\right|^{2}}+(1-p) \sigma_{\mathrm{d}}^{(3)} \frac{\left\langle E^{2}(\boldsymbol{r})|\boldsymbol{E}(\boldsymbol{r})|^{2}\right\rangle_{\mathrm{d}}}{E_{0}^{2}\left|E_{0}\right|^{2}}
$$

where the angular brackets $\langle\ldots\rangle_{\mathrm{m}}$ and $\langle\ldots\rangle_{\mathrm{d}}$ stand for the averaging over the metal and dielectric grains, respectively. Formula (6.17) for enhancement of the cubic nonlinearity in percolating composites was previously obtained by Aharony [8], Stroud and Hui [9] and Bergman [11]. Similar formula was independently obtained by Shalaev et al. to describe the Kerr enhancement in aggregates of metal particles [22-24]. The general equation Eq. (6.13) was derived in Ref. [43].

According to Eq. (6.16) the value of the Kerr enhancement $G_{\mathrm{K}}$ is proportional to the fourth power of the local field averaged over the sample. This is similar to the case of surface-enhanced Raman scattering with the enhancement factor $G_{\mathrm{RS}}$ given by Eq. (5.19). Note, however, that $G_{\mathrm{K}}$ is complex, whereas $G_{\mathrm{RS}}$ is a real positive quantity. Because the enhancement for Raman scattering is determined by the average of $|\boldsymbol{E}|^{4}$, which is phase insensitive, the upper limit for the enhancement is realized in this case.

The enhancement of the Kerr nonlinearity can be estimated analytically using the methods developed in Section 2. We consider first the case when $\sigma^{(3)}(\boldsymbol{r})$ in the dielectric component is of the same order of magnitude or larger than in the metal component. (The opposite case of almost linear dielectric $\left|\sigma_{\mathrm{d}}^{(3)}\right| \ll\left|\sigma_{\mathrm{m}}^{(3)}\right|$ will be considered below.) Then the Kerr enhancement $G_{\mathrm{K}}$ is estimated as

$$
\begin{aligned}
G_{\mathrm{K}} & \sim\left|\sigma_{\mathrm{e}}^{(3)} /\left\langle\sigma^{(3)}(\boldsymbol{r})\right\rangle\right|=\left|\varepsilon_{\mathrm{e}}^{(3)} /\left\langle\varepsilon^{(3)}(\boldsymbol{r})\right\rangle\right| \sim\left|M_{2,2}\right| \\
& \sim \rho\left(\xi_{\mathrm{A}} / a\right)^{d-8}\left(\frac{\left|\varepsilon_{\mathrm{m}}\right|}{\varepsilon_{\mathrm{d}}}\right)^{(2 v+s) /(t+s)}\left(\frac{\left|\varepsilon_{\mathrm{m}}\right|}{\varepsilon_{\mathrm{m}}^{\prime \prime}}\right)^{3},
\end{aligned}
$$

where Eq. (2.43) is used for the moment $M_{2,2}$ of the local field. For $\omega \ll \omega_{\mathrm{p}}$ the Kerr enhancement for $2 \mathrm{D}$ composites (semicontinuous metal films) is estimated as $G_{\mathrm{K}} \sim \rho\left(\xi_{\mathrm{A}} / a\right)^{d-8}\left(\omega_{\mathrm{p}} / \omega_{\tau}\right)^{3}$, where the Drude formula (2.1) is used for the metal dielectric constant $\varepsilon_{\mathrm{m}}$. For silver-on-glass semicontinuous films, Anderson localization length $\xi_{\mathrm{A}} \approx 2 a$ and density of states $\rho \approx 1$, the Kerr enhancement is estimated as $G_{\mathrm{K}} \sim 10^{5}-10^{6}$.

In Fig. 17 the results of numerical simulations for $G_{\mathrm{K}}$ are shown as a function of the metal filling factor $p$, for $d=2$. The plot has a two-peak structure, as in the case of Raman scattering. However, in contrast to $G_{\mathrm{RS}}$, the dip at $p=p_{c}$ is much stronger and at $p=p_{c}$ is proportional (as simulations 


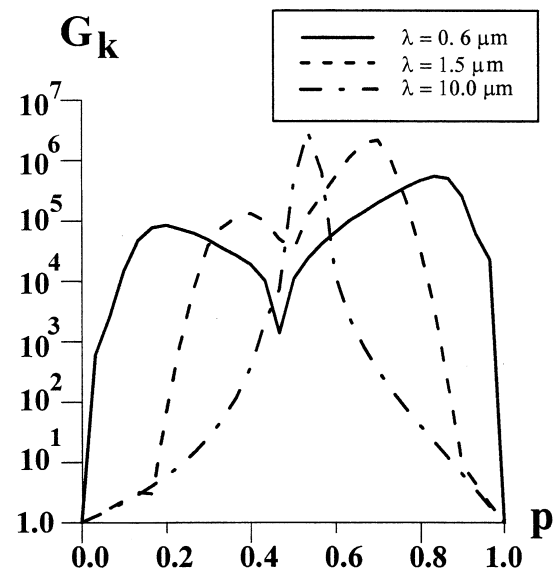

Fig. 17. Average enhancement of the Kerr optical nonlinearity $G_{\mathrm{K}}=\left|M_{2,2}\right|$ in silver semicontinuous films as a function of the metal concentration $p$ for three different wavelengths. The nonlinear Kerr permittivity $\varepsilon^{(3)}$ is the same for metal and dielectric components.

show) to the loss factor $\kappa$. This implies that at $p=p_{c}$, the enhancement is actually given by $G_{\mathrm{K}} \sim \kappa M_{2,2}$, where $M_{2,2}$ was found as above. This result might be a consequence of the special symmetry of a self-dual system at $p=p_{c}$. Formally, it could happen if the leading term in the power expansion of $M_{2,2}$ over $1 / \kappa$ cancels out because of the symmetry [see the discussion following Eq. (2.32)]. When this symmetry is somehow broken, e.g., by slightly moving away from the point $p=p_{c}$, the enhancement $G_{\mathrm{K}}$ increases and becomes $G_{\mathrm{K}} \sim\left|M_{2,2}\right| \sim G_{\mathrm{RS}} \sim M_{4,0}$, as seen in Fig. 17 . The fact that the minimum at $p=p_{c}$ is much less for SERS than for the Kerr process is probably related to the fact that the latter is a phase sensitive effect.

As shown in Section 2, the local field maxima are concentrated in the dielectric gaps when $\left|\varepsilon_{\mathrm{m}}\right| \gg \varepsilon_{\mathrm{d}}$. Therefore the enhancement estimate in Eq. (6.18) is valid when the Kerr nonlinearity is located mainly in these gaps (it can be due to the dielectric itself or due to adsorbed molecules).

Consider now the case when the Kerr nonlinearity is due to metal grains as in recent experiment $[16,140]$. Provided that $\varepsilon_{\mathrm{m}}^{\prime} \cong-\varepsilon_{\mathrm{d}}$, the local electric field are equally distributed in metal and dielectric components. Therefore the Kerr enhancement is still given by Eq. (6.18) where one should set $\left|\varepsilon_{\mathrm{m}}\right| / \varepsilon_{\mathrm{d}}=1$. The situation changes dramatically when $\left|\varepsilon_{\mathrm{m}}\right| \gg \varepsilon_{\mathrm{d}}$ since now the local field are concentrated in the dielectric gaps between the conducting clusters achieving the values $E_{\mathrm{m}}$ given by Eq. (2.35). The total current $J_{\mathrm{g}}$ of the electric displacement flowing in the dielectric gap between two resonate metal clusters of size $l_{\mathrm{r}}$ can be estimated as $J_{\mathrm{g}}=a E_{\mathrm{m}} \varepsilon_{\mathrm{e}} l_{\mathrm{r}}^{d-2}$, where $a E_{\mathrm{m}}$ is the voltage drop across the gap, $\varepsilon_{\mathrm{e}}$ is the effective dielectric constant of the composite. Because of the current continuity, the same current should flow in the adjacent metal clusters. In the metal cluster the current is concentrated in a percolating channel $[12,64]$. The electric field in the metal channel, which spans over the cluster, can be estimated as $E_{\mathrm{in}} \sim J_{\mathrm{g}} /\left(\varepsilon_{\mathrm{m}} a^{d-1}\right)$, where $a^{d-1}$ stands for the cross-section of the channel. Then the $n$th moment of the local electric field in a metal cluster of size $l_{\mathrm{r}}$ is equal to $\left\langle E_{\mathrm{in}}^{n}\right\rangle=E_{\mathrm{in}}^{n} \mathscr{L} a^{d-1} / l_{\mathrm{r}}^{d}$, where $\mathscr{L} a^{d-1}$ is the volume of the conducting channel, $\mathscr{L}=a\left(\varepsilon_{\mathrm{m}} / \varepsilon_{\mathrm{e}}\right) l_{\mathrm{r}}^{-d+2}$ is the effective length of the conducting channel. Now let us take into account that only the fraction $\kappa=\varepsilon_{\mathrm{m}}^{\prime \prime} /\left|\varepsilon_{\mathrm{m}}\right| \ll 1$ of metal clusters of size $l_{\mathrm{r}}$ are excited by the external electric 


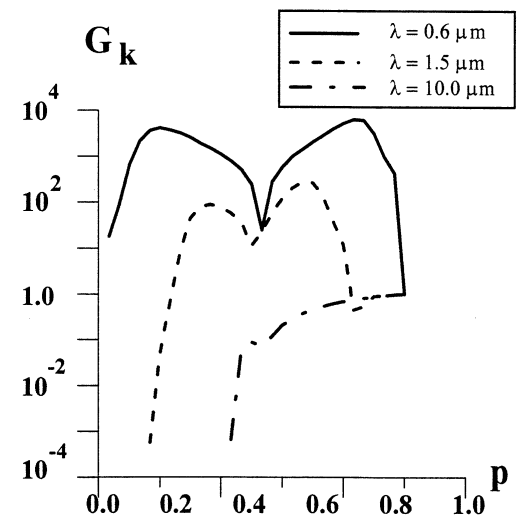

Fig. 18. Average enhancement of the Kerr optical nonlinearity $G_{\mathrm{K}}^{\text {metal }}=\left|M_{2,2}^{\text {metal }}\right|$ in silver semicontinuous films as a function of the metal concentration $p$ for three different wavelengths. The nonlinear Kerr permittivity $\varepsilon^{(3)}$ is in the metal component only.

field. Then the following estimate is obtained for the moments $M_{n}^{\text {met }}=\left\langle|E|^{n}\right\rangle_{\text {met }} / E_{0}^{n}=\kappa\left\langle E_{\text {in }}^{n}\right\rangle / E_{0}^{n}$ of the electric field in the metal component

$$
M_{n}^{\mathrm{met}} \sim\left(\frac{\left|\varepsilon_{\mathrm{m}}\right|}{\varepsilon_{\mathrm{m}}^{\prime \prime}}\right)^{n-1}\left(\frac{\left|\varepsilon_{\mathrm{m}}\right|}{\varepsilon_{\mathrm{d}}}\right)^{[(d-1)(n-2) v-t(n-1)] /(t+s)},
$$

where the expression (2.34) is used for the size $l_{\mathrm{r}}$ of the resonant clusters. The corresponding enhancement $G_{\mathrm{K}}^{\mathrm{met}}$ of the Kerr nonlinearity is estimated as

$$
G_{\mathrm{K}}^{\mathrm{met}} \sim M_{4}^{\mathrm{met}} \sim\left(\frac{\left|\varepsilon_{\mathrm{m}}\right|}{\varepsilon_{\mathrm{m}}^{\prime \prime}}\right)^{3}\left(\frac{\left|\varepsilon_{\mathrm{m}}\right|}{\varepsilon_{\mathrm{d}}}\right)^{[2(d-1) v-3 t] /(t+s)} .
$$

For two-dimensional systems $(d=2)$, where $t \cong s \cong v \cong 4 / 3$, Eq. (6.19) gives $G_{\mathrm{K}}^{\text {met }} \sim M_{4}^{\text {met }} \sim$ $\left(\left|\varepsilon_{\mathrm{m}}\right| / \varepsilon_{\mathrm{m}}^{\prime \prime}\right)^{3}\left(\varepsilon_{\mathrm{d}} /\left|\varepsilon_{\mathrm{m}}\right|\right)^{1 / 2}$.

Computer simulation results for enhancement of the Kerr nonlinearity $G_{\mathrm{K}}^{\mathrm{met}}$ for silver semicontinuous film are shown in Fig. 18 as a function of the metal concentration $p$. From Figs. 17 and 18 it follows that $G_{\mathrm{K}}^{\text {met }} \ll G_{\mathrm{K}}$ as expected. Near the percolation threshold, we can compute the Kerr enhancements $G_{\mathrm{K}}^{\mathrm{met}}$ and $G_{\mathrm{K}}$ quantitatively. For the $2 \mathrm{D}$ case, where $t \approx s \approx v$, Eqs. (6.18) and (6.19) give

$$
\frac{G_{\mathrm{K}}}{G_{\mathrm{K}}^{\mathrm{met}}} \sim\left(\frac{\left|\varepsilon_{\mathrm{m}}\right|}{\varepsilon_{\mathrm{d}}}\right)^{2} .
$$

Since in optic and infrared spectral ranges $\left|\varepsilon_{\mathrm{m}}\right| \gg \varepsilon_{\mathrm{d}}$, the Kerr nonlinearity enhancement is much larger when the "seed" nonlinearity is located in the dielectric gaps, where the local fields are much larger than in metal. It follows from Eq. (6.21) and also from Fig. 18 that the Kerr enhancement $G_{\mathrm{K}}^{\mathrm{met}}$ may become less than one. This means that local electric fields in the metal component can be smaller than the external field on average. For semicontinuous silver films on a glass substrate it occurs for wavelength $\lambda>10 \mu \mathrm{m}$ as one can see in Fig. 18. 
Note also that enhancement for nearly degenerate four-wave mixing $G_{\mathrm{FWM}}$, such as coherent anti-Stokes Raman scattering (CARS) and optical phase conjugation (OPC) process, is estimated as $G_{\mathrm{FWM}} \sim\left|G_{\mathrm{K}}\right|^{2}$ and can be very large on average and, especially, in the hot spots [15].

\subsection{Percolation-enhanced nonlinear scattering from metal-dielectric films}

Nonlinear optical processes of the $n$th order are proportional to $E^{n}(\boldsymbol{r})$ and, therefore, the strong spatial fluctuations of the "nonlinear" field source, $\propto E^{n}(r)$, can result in giant nonlinear scattering from a composite material.

In this subsection we consider percolation-enhanced nonlinear scattering (PENS) from a random metal-dielectric film (also referred to as a semicontinuous metal film) at the metal filling factor $p$ close to the percolation threshold, $p=p_{c}$. Specifically, the enhanced nonlinear scattering, which is due to local field oscillation at frequency $n \omega$, is studied; while a percolation metal-dielectric film is illuminated by an em wave of frequency $\omega$.

At the percolation, an infinite metal cluster spans over the entire sample and the metal-dielectric transition occurs in a semicontinuous metal film $[12,64]$. Optical excitations of the self-similar fractal clusters formed by metal particles near $p_{c}$ result in giant, scale-invariant, field fluctuations that makes the considered here PENS differ from the known phenomena of surface polariton excitations and harmonic generation from smooth and rough metal surfaces (see [115-119,60, 120-123] and references therein).

It was shown in Section 4 that while Rayleigh scattering is strongly enhanced it is still only a small correction to the specular reflection and transmission. In contrast, below it will be shown that PENS with a broad angular distribution can be a leading optical process.

For simplicity, it is assumed that a semicontinuous film is illuminated by the light propagating normal to the film, with the wavelength $\lambda$ larger than any intrinsic spatial scale in the film. The gaps between metal grains are filled by the dielectric substrate so that a semicontinuous metal film can be thought of as a two-dimensional array of metal and dielectric grains that are randomly distributed over a plane. For an incident wave of frequency $\omega, n$th harmonic generation $(n \mathrm{HG})$ is considered in a semicontinuous metal film.

It is assumed that a semicontinuous metal film is covered by a layer possessing the nonlinear conductivity $\sigma^{(n)}$ that results in $n \mathrm{HG}$ (e.g., it can be a layer of nonlinear organic molecules, semiconductor quantum dots, or a quantum well on top of a percolation film). The local electric field $\boldsymbol{E}_{\omega}(\boldsymbol{r})$ induced in the film by the external field $\boldsymbol{E}_{0}$ generates in the layer the $n \omega$ current $\sigma^{(n)} \boldsymbol{E}_{\omega} E_{\omega}^{n-1}$. (This expression, strictly speaking, holds only for the scalar nonlinear conductivity and odd $n$ (i.e., $n=2 k+1$ ), when $E^{n-1}=(\boldsymbol{E} \cdot \boldsymbol{E})^{k}$. However, for estimates, the formula can be used in the general case, for arbitrary $n$.) We would also point out that in this subsection the external field, oscillated at frequency $\omega$, is still denoted as $\boldsymbol{E}_{0}$ though the frequency is indicated explicitly for other fields. The nonlinear current $\sigma^{(n)} \boldsymbol{E}_{\omega} E_{\omega}^{n-1}$, in turn, interacts with the film and generates the "seed" $n \omega$ electric field, with the amplitude $\boldsymbol{E}^{(n)}=\sigma^{(n)} E_{\omega}^{n-1} \boldsymbol{E}_{\omega} / \sigma^{(1)}$, where $\sigma^{(1)}$ is the linear conductivity of the nonlinear layer at frequency $n \omega$. The electric field $\boldsymbol{E}^{(n)}$ can be thought of as an inhomogeneous external field exciting the film at $n \omega$ frequency.

The $n \mathrm{HG}$ current $\boldsymbol{j}^{(n)}$ induced in the film by the "seed" field $\boldsymbol{E}^{(n)}$ can be found in terms of the nonlocal conductivity matrix $\hat{\Sigma}\left(\boldsymbol{r}, \boldsymbol{r}^{\prime}\right)$ introduced in Section 5.1 by Eq. (5.1). The nonlocal 
conductivity relates the applied (external) field at point $\boldsymbol{r}^{\prime}$ to the current at point $\boldsymbol{r}$,

$$
j_{\beta}^{(n)}(\boldsymbol{r})=\int \sum_{\beta \alpha}^{(n)}\left(\boldsymbol{r}, \boldsymbol{r}^{\prime}\right) E_{\alpha}^{(n)}\left(\boldsymbol{r}^{\prime}\right) \mathrm{d} \boldsymbol{r}^{\prime},
$$

where $\Sigma_{\beta \alpha}^{(n)}$ is the conductivity matrix at frequency $n \omega$ and the integration is over the entire film area [15]. The Greek indices take values $\{x, y\}$ and summation over repeated indices is implied. It is the current $\boldsymbol{j}^{(n)}$ that eventually generates the nonlinear scattered field at frequency $n \omega$.

Using the numerical technique described in Section 3.1, the spatial distribution of the local-field can be calculated. For example, in Fig. 3 it is shown the normalized real part of the $3 \omega$ local field $\operatorname{Re}\left[E^{2}(\boldsymbol{r}) E_{x}(\boldsymbol{r})\right] /\left|E_{0}\right|^{3}$ in a $2 \mathrm{D}$ silver-on-glass film at $p=p_{c}$ and $\lambda=1.5 \mu \mathrm{m}$ [104]. As seen in Fig. 3, the fluctuating $3 \omega$ fields form a set of sharp peaks, looking up and down, and having the magnitudes $\sim 10^{4}-10^{6}$. Such huge fluctuations of the local fields are anticipated to trigger the PENS at frequency $3 \omega$. The larger the number $n$ of the harmonic, the stronger the fluctuation of the corresponding $n \omega$ local field. Therefore, PENS becomes progressively more pronounced for higher harmonics.

By using the standard approach of the scattering theory [65] adopted to semicontinuous metal films in [44] (see Section 4.1) and assuming that the incident light is unpolarized, the integral scattering is obtained in all directions but the specular one is

$$
S=\left(4 k^{2} / 3 c\right) \int\left(\left\langle j_{\alpha}^{(n)}\left(\boldsymbol{r}_{1}\right) j_{\alpha}^{(n) *}\left(\boldsymbol{r}_{2}\right)\right\rangle-\left|\left\langle\boldsymbol{j}^{(n)}\right\rangle\right|^{2}\right) \mathrm{d} \boldsymbol{r}_{1} \mathrm{~d} \boldsymbol{r}_{2},
$$

where the integrations is over the entire are a $A$ of the film, $k=\omega / c$ and the angular brackets stand for the ensemble average. As in Section 4.1, it is assumed that the integrand vanishes for distances $r \ll \lambda$, where $\boldsymbol{r}=\boldsymbol{r}_{2}-\boldsymbol{r}_{1}$; therefore, the retardation term $\sim \exp (\mathrm{i} \boldsymbol{k} \cdot \boldsymbol{r})$ is omitted [see discussion below Eq. (4.6)]. Using Eq. (6.22), the current-current correlator can be rewritten in Eq. (6.23) as

$$
\int\left\langle j_{\alpha}^{(n)}\left(\boldsymbol{r}_{1}\right) j_{\alpha}^{(n) *}\left(\boldsymbol{r}_{2}\right)\right\rangle \mathrm{d} \boldsymbol{r}_{1} \mathrm{~d} \boldsymbol{r}_{2}=\int\left\langle\sum_{\gamma \beta}^{(n)}\left(\boldsymbol{r}_{1}, \boldsymbol{r}_{3}\right) \sum_{\delta \alpha}^{(n) *}\left(\boldsymbol{r}_{2}, \boldsymbol{r}_{4}\right) \delta_{\gamma \delta}\left\langle E_{\beta}^{(n)}\left(\boldsymbol{r}_{3}\right) E_{\alpha}^{*(n)}\left(\boldsymbol{r}_{4}\right)\right\rangle_{0}\right\rangle \prod_{i=1}^{4} \mathrm{~d} \boldsymbol{r}_{i},
$$

where $\langle\cdots\rangle_{0}$ denotes the averaging over the light polarizations. For further consideration the spatial uniform "probe" field $\boldsymbol{E}_{n \omega}^{(0)}$ is introduced. This field oscillates at frequency $n \omega$ and is supposed to be unpolarized. For the unpolarized light the following equation holds $\delta_{\gamma \delta}=2\left\langle E_{n \omega, \gamma}^{(0)} E_{n \omega, \delta}^{(0) *}\right\rangle_{0} /\left|E_{n \omega}^{(0)}\right|^{2}$. This expression is substituted in Eq. (6.24). Then integration over the coordinates $\boldsymbol{r}_{1}, \boldsymbol{r}_{2}$, and averaging over independent polarizations of fields $E_{n \omega}^{(0)}, E_{0}$ gives the following equation for the current-current correlation function:

$$
\begin{aligned}
& \int\left\langle j_{\alpha}^{(n)}\left(\boldsymbol{r}_{1}\right) j_{\alpha}^{(n) *}\left(\boldsymbol{r}_{2}\right)\right\rangle \mathrm{d} \boldsymbol{r}_{1} \mathrm{~d} \boldsymbol{r}_{2} \\
& =\frac{1}{\left|E_{n \omega}^{(0)}\right|^{2}} \int\left\langle\sigma_{n \omega}\left(\boldsymbol{r}_{3}\right) \sigma_{n \omega}^{*}\left(\boldsymbol{r}_{4}\right)\left(\boldsymbol{E}_{n \omega}\left(\boldsymbol{r}_{3}\right) \cdot \boldsymbol{E}_{n \omega}^{*}\left(\boldsymbol{r}_{4}\right)\right)\left(\boldsymbol{E}^{(n)}\left(\boldsymbol{r}_{3}\right) \cdot \boldsymbol{E}^{(n) *}\left(\boldsymbol{r}_{4}\right)\right)\right\rangle \mathrm{d} \boldsymbol{r}_{3} \mathrm{~d} \boldsymbol{r}_{4},
\end{aligned}
$$

where $E_{n \omega}(r)$ is the local $n \omega$ field excited in the film by "probe" field $E_{n \omega}^{(0)}, \sigma_{n \omega}(r)$ is the film linear conductivity at frequency $n \omega$.

In macroscopically homogeneous and isotropic films considered here, the current correlator given by Eq. (6.25) does not depend on direction of the probe field $E_{n \omega}^{(0)}$. Therefore the field $\boldsymbol{E}_{n \omega}^{(0)}$ can 
be chosen now to be collinear with external field $\boldsymbol{E}_{0}$. In macroscopically isotropic films, considered here, the average nonlinear current $\left\langle\boldsymbol{j}^{(n)}\right\rangle$ is parallel to the external field $\boldsymbol{E}_{0}$. If the probe field $\boldsymbol{E}_{n \omega}^{(0)}$ is aligned with $\boldsymbol{E}_{0}$ the square of the nonlinear current is equal to $\left|\left\langle\boldsymbol{j}^{(n)}\right\rangle\right|^{2}=\left|\left\langle\boldsymbol{E}_{n \omega}^{(0)} \cdot \boldsymbol{j}^{(n)}\right\rangle\right|^{2} /\left|E_{n \omega}^{(0)}\right|^{2}$. Then using Eq. (6.22) the second term in the right-hand side of Eq. (6.23) can be rewritten as

$$
\left|\left\langle\boldsymbol{j}^{(n)}\right\rangle\right|^{2}=\frac{1}{A\left|E_{n \omega}^{(0)}\right|^{2}}\left|\int E_{n \omega, \beta}^{(0)} \sum_{\beta \alpha}^{(n)}\left(\boldsymbol{r}_{1}, \boldsymbol{r}_{2}\right) E_{\alpha}^{(n)}\left(\boldsymbol{r}_{2}\right) \mathrm{d} \boldsymbol{r}_{1} \mathrm{~d} \boldsymbol{r}_{2}\right|^{2},
$$

where the integrations are over the entire film area $A$. Integration over coordinate $\boldsymbol{r}_{1}$ in Eq. (6.26) gives for the average nonlinear current the following expression:

$$
\left|\left\langle\boldsymbol{j}^{(n)}\right\rangle\right|^{2}=\frac{\left|\left\langle\sigma_{n \omega}\left(\boldsymbol{E}_{n \omega} \cdot \boldsymbol{E}^{(n)}\right)\right\rangle\right|^{2}}{\left|E_{n \omega}^{(0)}\right|^{2}} .
$$

The substitution of Eqs. (6.25) and (6.27) in Eq. (6.23) results in

$$
S=\frac{8 \pi k^{2}}{3 c\left|\boldsymbol{E}_{n \omega}^{(0)}\right|^{2}}\left|\frac{\sigma^{(n)}}{\sigma^{(1)}}\right|^{2} A\left\langle\left|\sigma \boldsymbol{E}_{n \omega}\right|^{2}\left|\boldsymbol{E}_{\omega}\right|^{2}\left|E_{\omega}\right|^{2(n-1)}\right\rangle \int_{0}^{\infty} g^{(n)}(r) r \mathrm{~d} r,
$$

where $g^{(n)}(r)$ is the nonlinear correlation function defined as

$$
g^{(n)}(r)=\frac{\left\langle\sigma_{n \omega}\left(\boldsymbol{r}_{1}\right) \sigma_{n \omega}^{*}\left(\boldsymbol{r}_{2}\right)\left[\boldsymbol{E}_{n \omega}\left(\boldsymbol{r}_{1}\right) \cdot \boldsymbol{E}_{n \omega}^{*}\left(\boldsymbol{r}_{2}\right)\right]\left[\boldsymbol{E}^{(n)}\left(\boldsymbol{r}_{1}\right) \cdot \boldsymbol{E}^{(n) *}\left(\boldsymbol{r}_{2}\right)\right]\right\rangle-\left|\left\langle\sigma_{n \omega}\left(\boldsymbol{E}^{(n)} \cdot \boldsymbol{E}_{n \omega}\right)\right\rangle\right|^{2}}{\left\langle\left|\sigma_{n \omega} \boldsymbol{E}_{n \omega}\right|^{2}\left|\boldsymbol{E}^{(n)}\right|^{2}\right\rangle},
$$

which depends on the distance $r=\left|\boldsymbol{r}_{1}-\boldsymbol{r}_{2}\right|$ between points $\boldsymbol{r}_{1}$ and $\boldsymbol{r}_{2}$ for macroscopically homogeneous and isotropic films.

Thus obtained PENS $S$ is compared with the $n \omega$ signal $I_{n \omega}$ from the nonlinear layer on a dielectric film with no metal grains on it, $I_{n \omega}=\left(c \varepsilon_{\mathrm{d}}^{2} / 2 \pi\right) A\left|\sigma^{(n)} / \sigma^{(1)}\right|^{2}\left|E_{\omega}^{(0)}\right|^{2}\left|E_{\omega}^{(0)}\right|^{2(n-1)}$. The enhancement factor for PENS, $G^{(n)}=S / I_{n \omega}$ is expressed in terms of the local dielectric constant $\varepsilon_{n \omega}$ at frequency $n \omega$ as

$$
G^{(n)}=\frac{(k a)^{4}}{3} \frac{\left\langle\left.\varepsilon_{n \omega} \boldsymbol{E}_{n \omega}\right|^{2}\left|\boldsymbol{E}_{\omega}\right|^{2}\left|E_{\omega}\right|^{2(n-1)}\right\rangle}{\varepsilon_{\mathrm{d}}^{2}\left|\boldsymbol{E}_{n \omega}^{(0)}\right|^{2}\left|\boldsymbol{E}_{0}\right|^{2}\left|E_{0}\right|^{2(n-1)}} \frac{n^{2}}{a^{2}} \int_{0}^{\infty} g^{(n)}(r) r \mathrm{~d} r .
$$

Note that for a homogeneous $(p=0$ and $p=1)$ surface $g^{(n)}(r)=0$ and, therefore, $G^{(n)}=0$, so that the scattering occurs in the reflected direction only. Besides the small factor $(k a)^{4}$, which is similar to that in the standard linear Rayleigh scattering, the enhancement $G^{(n)}$ for PENS is proportional to the $2(n+1)$ power of the local field $E: G^{(n)} \sim\left\langle|E|^{2(n+1)}\right\rangle$. For highly fluctuating local fields, this factor can be very large (see Fig. 20). To understand the origin of the giant PENS the field fluctuations are considered below in more detail.

A metal-dielectric transition occurring at the percolation threshold to some extent is similar to a second-order phase transition [64]. In this case one could anticipate that local field fluctuations are rather large and have long-range correlations near the percolation threshold $p_{c}[8,9,11,12]$. What might be more surprising is that the field fluctuations in the optical spectral range discussed above are quite different from those for a second-order phase transition.

The local electric field fluctuations resulting in PENS are of the resonant character and the local field variation can be several orders of magnitude. In accordance with this, the field correlation 


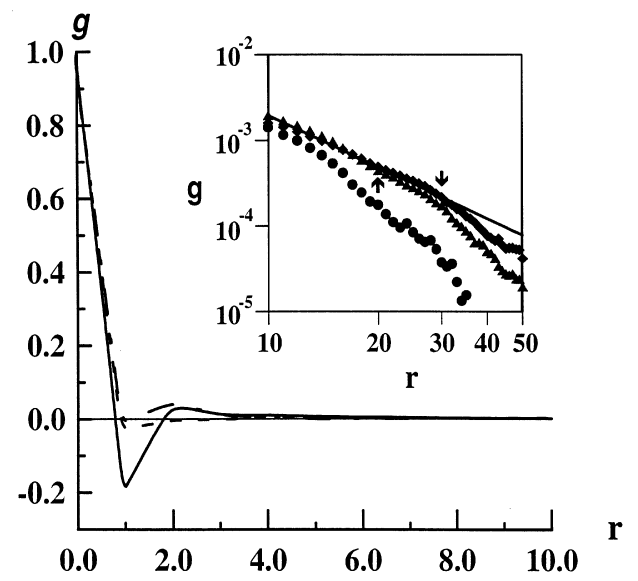

Fig. 19. Correlation function $g^{(3)}(r)$, for silver on glass semicontinuous film at the percolation threshold $p=p_{c}$. Different curves correspond to $\lambda_{1}=0.34 \mu \mathrm{m}$ (solid line and circles, in the inset), $\lambda_{2}=0.53 \mu \mathrm{m}$ (dashed line and triangles), and $\lambda_{3}=0.9 \mu \mathrm{m}$ (point-dashed line and diamonds); the arrows are theoretical estimates for $\xi_{\mathrm{e}}\left(\lambda_{2}\right)$ and $\xi_{\mathrm{e}}\left(\lambda_{3}\right)$. The straight line illustrates the scaling dependence of $g^{(3)}(r)$ in the tail. The units in which $a_{0}=1$ are used.

function $g^{(3)}(r)$, shown in Fig. 19 for the silver on glass semicontinuous film, drops very rapidly for $r>a$, and has a negative minimum, regardless of the magnitude of the local field correlation length $\xi_{\mathrm{e}}$; the anticorrelation occurs because the field maxima have different signs, as seen in Fig. 3. The power-low decrease of $g^{(3)}(r)$, which is typical for critical phenomena, occurs in the tail only (see inset in Fig. 19).

The correlation function $g^{(3)}(r)$ deviates from the power-law (the straight line in Fig. 19) for distances $r$ larger than field correlation length $\xi_{\mathrm{e}}$ introduced in Section 2.3. The value of $\xi_{\mathrm{e}}$ can be estimated from Eq. (2.41) as $\xi_{\mathrm{e}}(\lambda) \simeq 5,20$ and 30 (in $a$ units) for $\lambda=0.34,0.53$ and $0.9 \mu \mathrm{m}$ respectively, which is in agreement with the calculations shown in the inset of Fig. 19 [45]. For a typical size of a metal grain in a semicontinuous film $a \simeq 2-20 \mathrm{~nm}$, the intrinsic spatial scale of the local field inhomogeneity $\xi_{\mathrm{e}} \ll \lambda$, as it has been assumed above. (Note that the quasistatic approximation does not work often in metal semicontinuous films due to the strong skin effect in metal grains as it is discussed in detail in Section 7). The integral of $g^{(3)}(r)$ in Eq. (6.30) is estimated as one, for all frequencies. Based on the above consideration the dimensionless integral $a^{-2} \int_{0}^{\infty} g^{(n)}(r) r \mathrm{~d} r$ is supposed to be of the order of one for all $n$.

From the spatial behavior of $g^{(n)}(r)$ and the field distribution shown in Fig. 3, one anticipates that in contrast to harmonic generation from "conventional" metal surfaces the PENS is characterized by a broad-angle distribution, with the integral (over all directions) scattering much larger than the coherent scattering in the reflected direction.

The diffusive scattering of the second harmonic from the metal-dielectric film has been observed in experiments with $C_{60}$-coated semicontinuous silver films [124]. The diffusely scattered second harmonic generation was also observed from thin but continuous silver films [125] on which surface plasmon mode was excited in the Kretschmann geometry. This effect had no proper theoretical description. We believe that the diffusive scattering of $2 \omega$ field can be attributed to the 
anomalous fluctuations of local electric fields on the roughness features with spatial scale $a$ much smaller than wavelength $\lambda$ of the incident light. Then the scattering observed in the experiment [125] is similar to the considered here PENS from percolation films.

To estimate PENS quantitatively note that the typical size $l_{\mathrm{r}}(\omega) \sim a \sqrt{\left|\varepsilon_{\mathrm{m}}(\omega)\right|}$ of the local field maxima [see discussion at Eq. (2.42)] increases with decreasing frequency. Thus for a Drude metal $l_{\mathrm{r}}(\omega) \propto \omega^{-1}$ when $\omega \ll \omega_{\mathrm{p}}$. Since the spatial scales for the field $\boldsymbol{E}_{n \omega}$ and $\boldsymbol{E}_{\omega}$ are different the average $\left\langle\left(\left|\boldsymbol{E}_{n \omega}\right|^{2}\left|\boldsymbol{E}_{\omega}\right|^{2}\left|E_{\omega}\right|^{2(n-1)}\right)^{2}\right\rangle$ in Eq. (6.30) might be decoupled and approximated roughly as $\left\langle\left(\left|\varepsilon_{n \omega} \boldsymbol{E}_{n \omega}\right|^{2}\left|\boldsymbol{E}_{\omega}\right|^{2}\left|E_{\omega}\right|^{2(n-1)}\right)^{2}\right\rangle \sim\left\langle\left|\varepsilon_{n \omega} E_{n \omega}\right|^{2}\right\rangle\left\langle\left|E_{\omega}\right|^{2 n}\right\rangle \sim\left|\varepsilon_{n \omega} \varepsilon_{\mathrm{d}}\right| M_{2, n \omega} M_{2 n}\left|E_{n \omega}^{(0)}\right|^{2}\left|E_{0}\right|^{2 n}$, where Eq. (4.17) is used for the average $\left\langle\left|\varepsilon_{n \omega} E_{n \omega}\right|^{2}\right\rangle, M_{2, n \omega}$ and $M_{2 n}$ are the spatial moments of the local fields $\boldsymbol{E}_{n \omega}$ and $\boldsymbol{E}_{\omega}$ correspondingly. This decoupling is substituted in Eq. (6.30). It is also taken into account that the integral there is of the order of unity as discussed above. Then Eq. (6.30) simplifies to

$$
\frac{G^{(n)}}{(k a)^{4}} \simeq C\left|\frac{\varepsilon_{\mathrm{m}}(n \omega)}{\varepsilon_{\mathrm{d}}}\right| M_{2, n \omega} M_{2 n},
$$

where $C$ is an adjustable pre-factor. Finally, using Eq. (2.45) for the moments $M_{2, n \omega}$ and $M_{2 n}$ and assuming that in this equation the localization length $\xi_{\mathrm{A}} \sim a$ and the density of states $\rho \sim 1$ the PENS factor $G^{(n)}$ for the $n$th harmonic is estimated as follows:

$$
\frac{G^{(n)}}{(k a)^{4}} \simeq C \frac{\left|\varepsilon_{\mathrm{m}}(n \omega)\right|^{5 / 2}\left|\varepsilon_{\mathrm{m}}(\omega)\right|^{3(n-1 / 2)}}{\varepsilon_{\mathrm{d}}^{n+1} \varepsilon_{\mathrm{m}}^{\prime \prime}(n \omega) \varepsilon_{\mathrm{m}}^{\prime \prime}(\omega)^{2 n-1}},
$$

where $C$ is an adjustable pre-factor. In transition to the second relation in Eq. (6.32) the generated frequency $n \omega$ is assumed as $n \omega<\omega_{\mathrm{p}}$ so that $\varepsilon_{\mathrm{m}}^{\prime}(n \omega)$ is negative; otherwise, $G^{(n)} \simeq C(k a)^{4} M_{\omega}^{(2 n)}$ since the local $n \omega$ fields are not enhanced for $\varepsilon_{\mathrm{m}}^{\prime}(n \omega)>0$. For the Drude metal and $\omega, n \omega \ll \omega_{\mathrm{p}}$, Eq. (6.32) can be simplified as

$$
G^{(n)} \sim C(k a)^{4} \frac{1}{\varepsilon_{\mathrm{d}}^{n+1}}\left(\frac{\omega_{\mathrm{p}}}{\omega_{\tau}}\right)^{2 n}\left(\frac{\omega_{\mathrm{p}}}{\omega}\right)^{2}
$$

i.e., PENS increases with increasing the order of a nonlinear process and decreases toward the infrared part of the spectrum as $G^{(n)} \propto \lambda^{-2}$, in contrast to the well known law $\lambda^{-4}$ for Rayleigh scattering. It is interesting to note that PENS is inversely proportional to the wavelength squared for high-harmonic scattering, independent of the order of optical nonlinearity.

In Fig. 20 the numerical results for the PENS factors $G^{(n)}$ are compared with predictions of the scaling formula (6.32), where the pre-factor $C \simeq 10^{-3}$ is used (note that $C$ is small because the above used decoupling is, of course, the upper estimate). For a very large spectral interval, there is good agreement between the developed scaling theory and numerical calculations. The PENS effect appears to be really huge, e.g., the enhancement for the fifth harmonic generation is $G^{(5)} /(k a)^{4} \sim 10^{21}$, for $\lambda=1.5 \mu \mathrm{m}$.

To summarize, large field fluctuations in random metal-dielectric composites near percolation result in a new physical phenomenon, percolation-enhanced nonlinear scattering (PENS), which is characterized by giant enhancement and a broad-angle distribution. 


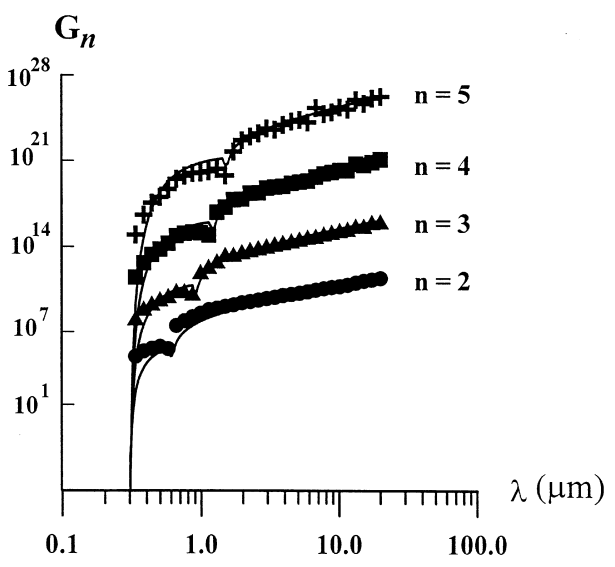

Fig. 20. The PENS factor $G^{(n)}$ for $n$-harmonic generation in a silver semicontinuous film at $p=p_{c}$. Numerical calculations for $n=2,3,4$ and $n=5$ are represented by $\boldsymbol{Q}, \mathbf{\Delta}, \mathbf{\square}$, and + , respectively.

\section{Electromagnetic properties of metal-dielectric crystals and composites: beyond the quasistatic approximation}

We consider now electromagnetic properties of metal-dielectric materials irradiated by a highfrequency electromagnetic field under conditions when the skin effect in metal grains is strong. Two different classes of metal-dielectric systems will be analyzed, percolation composites $[12,102]$ and artificial electromagnetic crystals $[62,63]$. The electromagnetic crystals are three-dimensional periodic structures of metal inclusions in a dielectric host. They are similar to the well known photonic crystals composed of periodic structures of dielectric particles. At high frequencies, when metal periodic stuctures can sustain plasmon excitations, they can also be referred to as plasmonic crystals.

Since metals have nonzero losses at the optical frequencies, most studies on photonic crystals are focused on dielectric structures. Nevertheless, metallic 3D structures, can find applications in the microwave range and, under some conditions, in the optical spectral range as well. Below the interaction of a cubic metal lattice with an electromagnetic field is considered.

Metal-dielectric percolation composites and 3D electromagnetic crystals are quite different objects at first glance. Still it is shown in this section that electromagnetic properties of random composites and electromagnetic crystals can be understood in terms of the effective dielectric constant and magnetic permeability, provided that the wavelength of an incident wave is much larger than an intrinsic spatial scale of the system. The wavelength inside a metal component can be very small. Most interesting effects are expected in the limit of the strong skin effect. Thus the consideration goes beyond the quasistatic approximation employed in the previous sections. It is important that methods for calculating the effective dielectric constant and magnetic permeability are essentially the same for composites and electromagnetic crystals. Moreover, the results for the effective parameters are also, to a large extent, similar. 


\subsection{Metal-dielectric composites}

The propagation of electromagnetic waves in percolation composites with wavelength $\lambda$ less than the percolation length $\xi_{\mathrm{p}}$, may be accompanied by strong scattering [see discussion in Section $6]$. On the other hand, the wave propagation at $\lambda \gg \xi_{\mathrm{p}}$ can be described by Maxwell's equations with effective dielectric constant $\varepsilon_{\mathrm{e}}$ and effective magnetic permeability $\mu_{\mathrm{e}}$. It will be discussed below (a) what sense have the effective parameters $\varepsilon_{\mathrm{e}}$ and $\mu_{\mathrm{e}}$ when the skin effect is strong and (b) how to express $\varepsilon_{\mathrm{e}}$ and $\mu_{\mathrm{e}}$ in terms of dielectric constants $\varepsilon_{\mathrm{m}}$ and $\varepsilon_{\mathrm{d}}$, magnetic permeabilities $\mu_{\mathrm{m}}$ and $\mu_{\mathrm{d}}$, and size $a$ of metal grains in a composite.

In order to calculate the effective parameters the approach suggested in Refs. $[107,126,127]$ is developed further.

When we are interested in the effective dielectric constant $\varepsilon_{\mathrm{e}}$ and effective magnetic permeability $\mu_{\mathrm{e}}$ the consideration can be restricted to the optically thin systems of size $\mathscr{L} \ll \lambda / \sqrt{\left|\varepsilon_{\mathrm{e}} \mu_{\mathrm{e}}\right|}$, which are still homogeneous from the percolation point of view $\left(\mathscr{L} \gg \xi_{\mathrm{p}}\right)$. Suppose that a percolation composite is placed inside of a resonator, where electromagnetic waves are excited. The effective parameters can be determined from the condition that the composite in the resonator results in the field change exactly like a geometrically equivalent homogeneous system with parameters $\varepsilon_{\mathrm{e}}$ and $\mu_{\mathrm{e}}$.

Let the electric or magnetic dipole mode be excited in the resonator. The effective dielectric (magnetic) constant $\varepsilon_{\mathrm{e}}\left(\mu_{\mathrm{e}}\right)$ can then be determined from a change in the characteristic frequency of the electric (magnetic) dipole oscillations. In the case of the electric dipole mode the system interacts with the electric component of an electromagnetic field, while in the magnetic dipole mode it interacts with the magnetic field. Note that in real experiments the effective parameters $\varepsilon_{\mathrm{e}}$ and $\mu_{\mathrm{e}}$ are measured using the same procedure [82, Section 90]. It is assumed, for simplicity, that the shape of the composite placed in a resonator is spherical. Then the electric and magnetic fields excited in the geometrically equivalent homogeneous system with the effective parameters $\varepsilon_{\mathrm{e}}$ and $\mu_{\mathrm{e}}$ are uniform and will be denoted as $\boldsymbol{E}_{0}$ and $\boldsymbol{H}_{0}$. It will be shown in this section how to obtain self-consistent equations for the parameters $\varepsilon_{\mathrm{e}}, \mu_{\mathrm{e}}$ and fields $\boldsymbol{E}_{0}$ and $\boldsymbol{H}_{0}$.

The change in the field when a composite is placed inside of the resonator, is determined by superposition of the fields scattered from individual metal and dielectric particles that have dielectric constants $\varepsilon_{\mathrm{m}}$ and $\varepsilon_{\mathrm{d}}$ respectively. The interaction between the particles is taken into account in the self-consistent approximation known as the effective medium theory $[12,49]$. In this theory, the interaction of a given metal or dielectric particle with the rest of the system is found by replacing the latter by a homogeneous medium with the effective parameters $\varepsilon_{\mathrm{e}}$ and $\mu_{\mathrm{e}}$. Assuming that the composite grains are spherical in shape, the electric fields $\boldsymbol{E}_{\mathrm{in}, \mathrm{m}}$ and $\boldsymbol{E}_{\mathrm{out}, \mathrm{m}}$, excited by electric field $\boldsymbol{E}_{0}$, are calculated inside and outside of a metal grain of size $a$. Thus the following equation (see [82, Section 59; 107,127]) is obtained for the electric field inside the metal grain:

$$
\boldsymbol{E}_{\mathrm{in}, \mathrm{m}}(\boldsymbol{r})=\boldsymbol{E}_{\mathrm{in}, \mathrm{m} 0}+4 \pi \boldsymbol{L}(\boldsymbol{r}),
$$

where

$$
\boldsymbol{E}_{\mathrm{in}, \mathrm{m} 0}=\frac{3 \varepsilon_{\mathrm{e}}}{2 \varepsilon_{\mathrm{e}}+\tilde{\varepsilon}_{\mathrm{m}}} \boldsymbol{E}_{0},
$$


$\tilde{\varepsilon}_{\mathrm{m}}$ is the renormalized dielectric constant of metal defined as

$$
\tilde{\varepsilon}_{\mathrm{m}}=\varepsilon_{\mathrm{m}} \frac{2 F\left(k_{\mathrm{m}} a\right)}{1-F\left(k_{\mathrm{m}} a\right)}, \quad F(x)=\frac{1}{x^{2}}-\frac{\cot x}{x},
$$

where $k=\omega / c$ is the wave vector in a free space, $k_{\mathrm{m}}=k \sqrt{\varepsilon_{\mathrm{m}} \mu_{\mathrm{m}}}$, and $a$ is the radius of a metal grain. The skin (penetration) depth $\delta$ is equal to $\delta=1 / \operatorname{Im} k_{\mathrm{m}}$. When metal conductivity $\sigma_{\mathrm{m}}$ is a real quantity (microwave and radio frequency) the skin depth $\delta=c / \sqrt{2 \pi \mu_{\mathrm{m}} \sigma_{\mathrm{m}} \omega}$. In the Cartesian coordinate system with the " $z$ " axis directed along the field $\boldsymbol{E}_{0}$, the local electric field $\boldsymbol{L}$ in Eq. (7.1) is determined by the equation

$$
\operatorname{curl} \boldsymbol{L}(\boldsymbol{r})=\frac{1}{4 \pi} \operatorname{curl} \boldsymbol{E}_{\mathrm{in}, \mathrm{m}}(\boldsymbol{r})=\frac{\mathrm{i} k}{4 \pi} \boldsymbol{B}_{E},
$$

where the loop field

$$
\boldsymbol{B}_{E}=-3 \mathrm{i} E_{0} \frac{a k \varepsilon_{\mathrm{m}} \varepsilon_{\mathrm{e}} \sin \left(k_{\mathrm{m}} r\right) F\left(k_{\mathrm{m}} r\right)}{\left(2 \varepsilon_{\mathrm{e}}+\tilde{\varepsilon}_{\mathrm{m}}\right) \sin \left(k_{\mathrm{m}} a\right)\left(F\left(k_{\mathrm{m}} a\right)-1\right)}\left\{\frac{y}{r},-\frac{x}{r}, 0\right\}
$$

is a rotational magnetic induction arising in a metal particle from the skin effect. Therefore the inside electric field consists of curl-free part $\boldsymbol{E}_{\mathrm{in}, \mathrm{m} 0}$ and the rotational part $\boldsymbol{L}(\boldsymbol{r})$ that depends on the coordinate. The field outside the metal particle equals

$$
\boldsymbol{E}_{\mathrm{out}, \mathrm{m}}=\boldsymbol{E}_{0}+a^{3} \frac{\varepsilon_{\mathrm{e}}-\tilde{\varepsilon}_{\mathrm{m}}}{2 \varepsilon_{\mathrm{e}}+\tilde{\varepsilon}_{\mathrm{m}}} \nabla\left(\frac{\boldsymbol{E}_{0} \cdot \boldsymbol{r}}{r^{3}}\right)
$$

i.e., it also depends on the renormalized dielectric function $\varepsilon_{\mathrm{m}}$. The local wavelength inside a dielectric grain $\lambda_{\mathrm{d}}=\lambda / \sqrt{\varepsilon_{\mathrm{d}}}$ is assumed to be much larger than the grain size: $\lambda_{\mathrm{d}} \gg a$. Then the electric fields inside and outside a dielectric particle are given by the well known equations (see [82, Section 8])

$$
\boldsymbol{E}_{\mathrm{in}, \mathrm{d}}=\boldsymbol{E}_{0} \frac{3 \varepsilon_{\mathrm{e}}}{2 \varepsilon_{\mathrm{e}}+\varepsilon_{\mathrm{d}}}
$$

and

$$
\boldsymbol{E}_{\text {out }, \mathrm{d}}=\boldsymbol{E}_{0}+a^{3} \frac{\varepsilon_{\mathrm{e}}-\varepsilon_{\mathrm{d}}}{2 \varepsilon_{\mathrm{e}}+\varepsilon_{\mathrm{d}}} \nabla\left(\frac{\boldsymbol{E}_{0} \cdot \boldsymbol{r}}{r^{3}}\right) .
$$

Similar equations can be obtained for the magnetic field excited by uniform magnetic field $\boldsymbol{H}_{0}$ inside and outside a metal (dielectric) particle:

$$
\boldsymbol{H}_{\mathrm{in}, \mathrm{m}}=\boldsymbol{H}_{\mathrm{in}, \mathrm{m} 0}+4 \pi \boldsymbol{M},
$$

where

$$
\boldsymbol{H}_{\mathrm{in}, \mathrm{m} 0}=\frac{3 \mu_{\mathrm{e}}}{2 \mu_{\mathrm{e}}+\tilde{\mu}_{\mathrm{m}}} \boldsymbol{H}_{0},
$$


and the renormalized metal magnetic permeability $\tilde{\mu}_{\mathrm{m}}$ equals

$$
\tilde{\mu}_{\mathrm{m}}=\mu_{\mathrm{m}} \frac{2 F\left(k_{\mathrm{m}} a\right)}{1-F\left(k_{\mathrm{m}} a\right)},
$$

where the function $F$ is defined in Eq. (7.3). Note that the renormalized metal magnetic permeability $\tilde{\mu}_{\mathrm{m}}$ is not equal to one, even if metal is nonmagnetic and the seed magnetic permeability $\mu_{\mathrm{m}}=1$. The local magnetic field $\boldsymbol{M}$ in Eq. (7.9) satisfies equations that are similar to Eqs. (7.4) and (7.5), namely

$$
\begin{aligned}
& \operatorname{curl} \boldsymbol{M}=\frac{1}{4 \pi} \operatorname{curl} \boldsymbol{H}_{\mathrm{in}, \mathrm{m}}=-\frac{\mathrm{i} k}{4 \pi} \boldsymbol{D}_{H}, \\
& \boldsymbol{D}_{H}=3 \mathrm{i} H_{0} \frac{a k \mu_{\mathrm{m}} \mu_{\mathrm{e}} \sin \left(k_{\mathrm{m}} r\right) F\left(k_{\mathrm{m}} r\right)}{\left(2 \mu_{\mathrm{e}}+\tilde{\mu}_{\mathrm{m}}\right) \sin \left(k_{\mathrm{m}} a\right)\left(F\left(k_{\mathrm{m}} a\right)-1\right)}\left\{\frac{y}{r},-\frac{x}{r}, 0\right\},
\end{aligned}
$$

where $\boldsymbol{D}$ is the electric displacement induced in the metal particle by high-frequency magnetic field $\boldsymbol{H}_{0}$. The displacement $\boldsymbol{D}$ can be written as $\boldsymbol{D}=\mathrm{i}(4 \pi / \omega) \boldsymbol{j}$, where the eddy electric currents $\boldsymbol{j}$ are known as Foucault currents. The field $\boldsymbol{H}_{\mathrm{in}, \mathrm{m} 0}$ is a potential part, while $\boldsymbol{M}$ is a rotational (solenoidal) part of the local magnetic field. The magnetic field outside the metal particle is irrotational (curl-free) and equals

$$
\boldsymbol{H}_{\mathrm{out}, \mathrm{m}}=\boldsymbol{H}_{0}+a^{3} \frac{\mu_{\mathrm{e}}-\tilde{\mu}_{\mathrm{m}}}{2 \mu_{\mathrm{e}}+\tilde{\mu}_{\mathrm{m}}} \nabla\left(\frac{\boldsymbol{H}_{0} \cdot \boldsymbol{r}}{r^{3}}\right) .
$$

It is supposed, for simplicity, that the dielectric component of the composite is nonmagnetic, i.e., the dielectric magnetic permeability $\mu_{\mathrm{d}}=1$. Then the magnetic fields inside and outside a dielectric particle are equal to

$$
\boldsymbol{H}_{\mathrm{in}, \mathrm{d}}=\boldsymbol{H}_{0} \frac{3 \mu_{\mathrm{e}}}{2 \mu_{\mathrm{e}}+1}
$$

and

$$
\boldsymbol{H}_{\text {out }, \mathrm{d}}=\boldsymbol{H}_{0}+a^{3} \frac{\mu_{\mathrm{e}}-1}{2 \mu_{\mathrm{e}}+1} \nabla\left(\frac{\boldsymbol{H}_{0} \cdot \boldsymbol{r}}{r^{3}}\right)
$$

respectively. The effective parameters $\varepsilon_{\mathrm{e}}$ and $\mu_{\mathrm{e}}$ are determined by the self-consistent condition that the scattered fields should vanish, when averaged over all inclusions, i.e., $\left\langle\boldsymbol{E}_{\text {out }}\right\rangle=p \boldsymbol{E}_{\text {out, } \mathrm{m}}+$ $(1-p) \boldsymbol{E}_{\text {out }, \mathrm{d}}=\boldsymbol{E}_{0}$ and $\left\langle\boldsymbol{H}_{\text {out }}\right\rangle=p \boldsymbol{H}_{\text {out }, \mathrm{m}}+(1-p) \boldsymbol{H}_{\text {out }, \mathrm{d}}=\boldsymbol{H}_{0}$, where $\langle\cdots\rangle$ stands for the volume averaging. Substituting here the scattered electric and magnetic fields from Eqs. (7.6), (7.8) and (7.14), (7.16) results in the following system:

$$
\begin{aligned}
& p \frac{\varepsilon_{\mathrm{e}}-\tilde{\varepsilon}_{\mathrm{m}}}{2 \varepsilon_{\mathrm{e}}+\tilde{\varepsilon}_{\mathrm{m}}}+(1-p) \frac{\varepsilon_{\mathrm{e}}-\varepsilon_{\mathrm{d}}}{2 \varepsilon_{\mathrm{e}}+\varepsilon_{\mathrm{d}}}=0, \\
& p \frac{\mu_{\mathrm{e}}-\tilde{\mu}_{\mathrm{m}}}{2 \mu_{\mathrm{e}}+\tilde{\mu}_{\mathrm{m}}}+(1-p) \frac{\mu_{\mathrm{e}}-1}{2 \mu_{\mathrm{e}}+1}=0
\end{aligned}
$$


that gives the effective dielectric constant $\varepsilon_{\mathrm{e}}$ and magnetic permeability $\mu_{\mathrm{e}}$ in terms of metal concentration $p$ and $\tilde{\varepsilon}_{\mathrm{m}}, \varepsilon_{\mathrm{d}}, \tilde{\mu}_{\mathrm{m}}$. Eqs. (7.17) and (7.18) are similar to equations of the traditional effective medium theory [12]. The skin effect results in renormalization of the dielectric constant and magnetic permeability of the conducting component; specifically, the metal dielectric constant $\varepsilon_{\mathrm{m}}$ and magnetic permeability $\mu_{\mathrm{m}}$ are replaced by $\tilde{\varepsilon}_{\mathrm{m}}$ and $\tilde{\mu}_{\mathrm{m}}$ given by Eqs. (7.3) and (7.11), respectively. This fact has a substantial effect on the frequency dependence of the effective parameters. For example, it is commonly accepted that the effective conductivity $\sigma_{\mathrm{e}}=-\mathrm{i} \omega \varepsilon_{\mathrm{e}} /(4 \pi)$ of a composite is dispersion-free, when the conductivity of metal component $\sigma_{\mathrm{m}}$ is frequency independent and large in comparison with frequency, $\sigma_{\mathrm{m}} \gg \omega$ (which is typical for the microwave and far-infrared ranges). Thus the traditional effective medium theory predicts that $\sigma_{\mathrm{e}}=\sigma_{\mathrm{m}}(3 p-1) / 2$ for the metal concentration $p$ sufficiently above the percolation threshold. Eq. (7.17) gives the same result for the effective conductivity $\sigma_{\mathrm{e}}$ but the metal conductivity is renormalized according to Eq. (7.3), which results in the following formula $\sigma_{\mathrm{e}}=\sigma_{\mathrm{m}} F\left(k_{\mathrm{m}} a\right)(3 p-1) /\left(1-F\left(k_{\mathrm{m}} a\right)\right)$. It follows from this expression that the effective conductivity has a dispersive behavior, provided that the skin effect in metal grains becomes important $[107,127]$. In the limit of very strong skin effect $\delta \ll a$ the effective conductivity decreases with frequency as $\sigma_{\mathrm{e}} \sim \sigma_{\mathrm{m}}(\delta / a) \sim \sigma_{\mathrm{m}} / \sqrt{\omega}$. Another interesting result is that percolation composites exhibit magnetic properties, even if they are absent in each component, i.e., if $\mu_{\mathrm{m}}=\mu_{\mathrm{d}}=1$ (see also discussion in Refs. $[126,128,129])$. In this case the real part $\mu_{\mathrm{e}}^{\prime}$ of the effective magnetic permeability $\mu_{\mathrm{e}}$ is less than one and it decreases with frequency. The imaginary part $\mu_{\mathrm{e}}^{\prime \prime}$ of the effective permeability has its maximum at frequencies such that $\delta \sim a$.

It will be shown now that the effective parameters $\varepsilon_{\mathrm{e}}$ and $\mu_{\mathrm{e}}$ determine propagation of an electromagnetic wave in the metal-dielectric composites. In the effective medium approximation, used here, the field is supposed to be the same in particular type of the grains. Therefore, the average electric field is equal to

$$
\langle\boldsymbol{E}\rangle=p \boldsymbol{E}_{\mathrm{in}, \mathrm{m}}+(1-p) \boldsymbol{E}_{\mathrm{in}, \mathrm{d}}=p \boldsymbol{E}_{\mathrm{in}, \mathrm{m} 0}+4 \pi\langle\boldsymbol{L}\rangle+(1-p) \boldsymbol{E}_{\mathrm{in}, \mathrm{d}},
$$

where Eq. (7.1) is used in transition to the second equality. When Eqs. (7.2) and (7.7) are substituted in Eq. (7.19), and Eq. (7.17) is taken into account, Eq. (7.19) simplifies to

$$
\langle\boldsymbol{E}\rangle=\boldsymbol{E}_{0}+4 \pi\langle\boldsymbol{L}\rangle,
$$

where $\langle\cdots\rangle$ denotes, as above, the volume average. Therefore, the irrotational part of the local field, being averaged over the volume, gives the field $\boldsymbol{E}_{0}$, while the second term in Eq. (7.20) results from the skin effect in metal grains. The above consideration being repeated for the average magnetic field $\langle\boldsymbol{H}\rangle$ [see Eqs. (7.9), (7.10) and (7.15)] results in

$$
\langle\boldsymbol{H}\rangle=p \boldsymbol{H}_{\mathrm{in}, \mathrm{m}}+(1-p) \boldsymbol{H}_{\mathrm{in}, \mathrm{d}}=\boldsymbol{H}_{0}+4 \pi\langle\boldsymbol{M}\rangle,
$$

where the rotational field $\boldsymbol{M}$ in metal grains is given by Eq. (7.12); the field $\boldsymbol{M}$ equals zero in dielectric grains. Again the average irrotational part of the local magnetic field gives $\boldsymbol{H}_{0}$, while the term $4 \pi\langle\boldsymbol{M}\rangle$ represents the average rotational (curl) magnetic field.

Consider now the average electric displacement $\langle\boldsymbol{D}\rangle$ induced in the system by electrical field $\boldsymbol{E}_{0}$. The displacement can be written as

$$
\langle\boldsymbol{D}\rangle=\varepsilon_{\mathrm{m}} p \boldsymbol{E}_{\mathrm{in}, \mathrm{m} 0}+4 \pi \varepsilon_{\mathrm{m}}\langle\boldsymbol{L}\rangle+(1-p) \varepsilon_{\mathrm{d}} \boldsymbol{E}_{\mathrm{in}, \mathrm{d}} .
$$


It follows from Eq. (7.2) for $\boldsymbol{E}_{\mathrm{in}, \mathrm{m} 0}$ and Eq. (7.4) for $\boldsymbol{L}$ that the sum $\varepsilon_{\mathrm{m}} p \boldsymbol{E}_{\mathrm{in}, \mathrm{m} 0}+4 \pi \varepsilon_{\mathrm{m}}\langle\boldsymbol{L}\rangle$ in Eq. (7.22) can be written as

$$
\begin{aligned}
\varepsilon_{\mathrm{m}} p \boldsymbol{E}_{\mathrm{in}, \mathrm{m} 0}+4 \pi \varepsilon_{\mathrm{m}}\langle\boldsymbol{L}\rangle=\varepsilon_{\mathrm{m}} p\left(\frac{3 \varepsilon_{\mathrm{e}}}{2 \varepsilon_{\mathrm{e}}+\tilde{\varepsilon}_{\mathrm{m}}} \boldsymbol{E}_{0}+\frac{4 \pi}{V} \int \boldsymbol{L} \mathrm{d} V\right) \\
=\varepsilon_{\mathrm{m}} p\left(\frac{3 \varepsilon_{\mathrm{e}}}{2 \varepsilon_{\mathrm{e}}+\tilde{\varepsilon}_{\mathrm{m}}} \boldsymbol{E}_{0}+\mathrm{i} \frac{k}{2 V} \int\left[\boldsymbol{r} \times \boldsymbol{B}_{E}\right] \mathrm{d} V\right)=p \frac{3 \varepsilon_{\mathrm{e}} \tilde{\varepsilon}_{\mathrm{m}}}{2 \varepsilon_{\mathrm{e}}+\tilde{\varepsilon}_{\mathrm{m}}} \boldsymbol{E}_{0},
\end{aligned}
$$

where the integration is over volume $V=4 \pi a^{3} / 3$ of a metal particle, and the field $\boldsymbol{B}_{E}$ is given by Eq. (7.5). Substituting Eqs. (7.23) and (7.7) in Eq. (7.22) gives

$$
\langle\boldsymbol{D}\rangle=\varepsilon_{\mathrm{e}} \boldsymbol{E}_{0},
$$

where Eq. (7.17) has been used. Therefore, the average electric displacement is proportional to the irrotational part of the local field averaged over the system and the coefficient is exactly equal to the effective dielectric constant. When the above consideration is repeated for the magnetic field it results in an equation for the average magnetic induction $\langle\boldsymbol{B}\rangle$, namely:

$$
\langle\boldsymbol{B}\rangle=\mu_{\mathrm{e}} \boldsymbol{H}_{0} .
$$

Eqs. (7.20) and (7.21) can be considered as definitions for fields $\boldsymbol{E}_{0}$ and $\boldsymbol{H}_{0}$. Indeed, if the local fields were known in the composite the fields $\boldsymbol{E}_{0}$ and $\boldsymbol{H}_{0}$ would be found from Eqs. (7.20) and (7.21). Then Eqs. (7.24) and (7.25) can be used to find the effective dielectric constant $\varepsilon_{\mathrm{e}}$ and effective magnetic permeability $\mu_{\mathrm{e}}$ of a composite. Eqs. (7.24) and (7.25) replace the usual constitutive equations $\langle\boldsymbol{D}\rangle=\varepsilon_{\mathrm{e}}\langle\boldsymbol{E}\rangle$ and $\langle\boldsymbol{B}\rangle=\mu_{\mathrm{e}}\langle\boldsymbol{H}\rangle$, which hold in the quasistatic case only.

We now proceed with derivation of equations for the macroscopic electromagnetism in metaldielectric composites. Eq. (7.24) gives the average electric displacement excited by the electric field $\boldsymbol{E}_{0}$, but the local magnetic field also excites eddy electric currents (Foucault currents). An addition of the electric displacement $\boldsymbol{D}_{H}$ given by Eq. (7.12) with the average displacement given by Eq. (7.24) gives the full electric displacement

$$
\langle\boldsymbol{D}\rangle_{\mathrm{f}}=\varepsilon_{\mathrm{e}} \boldsymbol{E}_{0}+\frac{\mathrm{i} 4 \pi}{k}\langle\operatorname{curl} \boldsymbol{M}\rangle,
$$

where it is still assumed that the system is much smaller than the wavelength $\lambda$. Note that the second term in Eq. (7.26) disappears when the skin effect vanishes, i.e., when $\left|k_{\mathrm{m}}\right| a \rightarrow 0$. Similarly, the average full magnetic induction $\langle\boldsymbol{B}\rangle_{\mathrm{f}}$ equals

$$
\langle\boldsymbol{B}\rangle_{\mathrm{f}}=\mu_{\mathrm{e}} \boldsymbol{H}_{0}-\frac{\mathrm{i} 4 \pi}{k}\langle\operatorname{curl} \boldsymbol{L}\rangle,
$$

where the vector $\boldsymbol{L}$ is given by Eq. (7.4). Now the Maxwell equations are averaged over a macroscopical volume $V$ whose size $\mathscr{L}$ is much larger than the percolation correlation length $\xi_{\mathrm{p}}$ but much smaller than the wavelength $\lambda, \xi_{\mathrm{p}} \ll \mathscr{L} \ll \lambda$. The volume $V$ is supposed to be centered in the point $\boldsymbol{r}$. The frequency domain is used. Thus the following Maxwell equations are obtained:

$$
\begin{aligned}
& \langle\operatorname{curl} \boldsymbol{E}\rangle=\mathrm{i} k\langle\boldsymbol{B}\rangle_{\mathrm{f}}=\mathrm{i} k \mu_{\mathrm{e}} \boldsymbol{H}_{0}+4 \pi\langle\operatorname{curl} \boldsymbol{L}\rangle, \\
& \langle\operatorname{curl} \boldsymbol{H}\rangle=-\mathrm{i} k\langle\boldsymbol{D}\rangle_{\mathrm{f}}=-\mathrm{i} k \varepsilon_{\mathrm{e}} \boldsymbol{E}_{0}+4 \pi\langle\operatorname{curl} \boldsymbol{M}\rangle,
\end{aligned}
$$


where Eqs. (7.26) and (7.27) are substituted for the electric and magnetic inductances respectively. The order of the curl operation and the volume average in Eqs. (7.28) and (7.29) can be changed as it is done usually for derivation of the macroscopic Maxwell equations (see [130, Chapter 6, Section 6.6]). For example, $\langle\operatorname{curl} \boldsymbol{E}\rangle$ can be written as $\langle\operatorname{curl} \boldsymbol{E}\rangle=\operatorname{curl}[\langle\boldsymbol{E}\rangle(\boldsymbol{r})]$, where the differentiation in 1.h.p. is over the position $\boldsymbol{r}$ of the volume $V$. Note that the fields $\boldsymbol{E}_{0}$ and $\boldsymbol{H}_{0}$ defined by Eqs. (7.20) and (7.21) are also functions of $\boldsymbol{r}$. Then the Maxwell equations (7.28) and (7.29) acquire the form

$$
\begin{aligned}
& \operatorname{curl} \boldsymbol{E}_{0}(\boldsymbol{r})=\mathrm{i} k \mu_{\mathrm{e}} \boldsymbol{H}_{0}(\boldsymbol{r}), \\
& \operatorname{curl} \boldsymbol{H}_{0}(\boldsymbol{r})=-\mathrm{i} k \varepsilon_{\mathrm{e}} \boldsymbol{E}_{0}(\boldsymbol{r}),
\end{aligned}
$$

i.e., they have the form typical for the macroscopic electromagnetism, describing, for example, propagation of electromagnetic waves in a composite media.

It is important that all quantities in Eqs. (7.20), (7.21), (7.24), (7.25), (7.30) and (7.31) are well defined and do not depend on the assumptions made in the course of their derivation. Thus the vector $\boldsymbol{M}$ in Eq. (7.21) can be determined as a magnetic moment of the eddy currents per unit volume, so that

$$
\langle\boldsymbol{M}\rangle=-\mathrm{i} \frac{k}{8 \pi V} \int\left[\boldsymbol{r} \times \boldsymbol{D}_{H}\right] \mathrm{d} V=\frac{1}{2 c V} \int\left[\boldsymbol{r} \times \boldsymbol{j}_{H}\right] \mathrm{d} V,
$$

where the integration now is over macroscopical volume $V$. This definition of vector $\boldsymbol{M}$ is in agreement with Eq. (7.12), but it is not required now that the eddy currents $\boldsymbol{j}_{H}$ are the same in all metal particles. In a similar way the vector $\boldsymbol{L}$ is defined as the spatial density of the "electric moments of magnetic eddy currents"

$$
\langle\boldsymbol{L}\rangle=\mathrm{i} \frac{k}{8 \pi V} \int\left[\boldsymbol{r} \times \boldsymbol{B}_{E}\right] \mathrm{d} V,
$$

where the integration is still over the volume $V$ and $\boldsymbol{B}_{E}=-(i 4 \pi / k) \operatorname{curl} \boldsymbol{E}$, with $\boldsymbol{E}$ being the local electric field. Note that the vector $\langle\boldsymbol{L}\rangle$ has no direct analog in the classical electrodynamics since there is no such thing as loop magnetic currents in atoms and molecules. After defining vectors $\boldsymbol{L}$ and $\boldsymbol{M}$, Eqs. (7.20), (7.21), (7.24), (7.25), (7.30) and (7.31) form a complete system of equations that determine the effective parameters and electrodynamic wave propagation in metal-dielectric composite media. Various approximations, such as the effective medium theory, can still be very useful in actual calculations of the effective parameters.

\subsection{Electromagnetic crystals}

In this subsection the method, discussed above, is used to calculate the effective dielectric constant and magnetic permeability of metal-dielectric crystals, known also as electromagnetic crystals. In these artificial crystals the metal component is assembled in a periodic lattice. Two limiting cases of the electromagnetic crystals are considered: a cubic lattice of unconnected metal spheres and three-dimensional conducting wire mesh configurated in a cubic lattice.

\subsubsection{Cubic lattice of metal spheres}

The local electromagnetic fields and effective parameters are considered for a system of metal spheres of radius $a$ that are embedded in a dielectric host (a vacuum, for example) at sites of a cubic 
lattice with period $\mathscr{L}>2 a$. The wavelength $\lambda$ of an incident electromagnetic wave is assumed to be much larger than the lattice period $\mathscr{L}$. Consider first the electric field distribution in the lattice cell centered at a metal sphere. The electric field outside the metal grain can be expanded in multipole series; for simplicity, the dipole approximation is used [131,132], which holds in the limit $\mathscr{L} \gg a$. In this approximation the outside field $\boldsymbol{E}_{\text {out }}$ has constant and dipole components only, namely

$$
\boldsymbol{E}_{\mathrm{out}}(\boldsymbol{r})=\boldsymbol{E}_{1}+B a^{3} \nabla\left(\frac{\boldsymbol{E}_{1} \cdot \boldsymbol{r}}{r^{3}}\right),
$$

where $\boldsymbol{E}_{1}$ is some electric field aligned with the electric field of the incident wave and $B$ is an unknown coefficient. Provided that the external field $\boldsymbol{E}_{\text {out }}$ is specified, the electric field $\boldsymbol{E}_{\text {in }}$ inside the metal grain can be found unambiguously by solving the Maxwell equations at the boundary conditions $\boldsymbol{E}_{\text {in }} \times \boldsymbol{n}=\boldsymbol{E}_{\text {out }} \times \boldsymbol{n}$ and $\varepsilon_{\mathrm{m}} \boldsymbol{E}_{\text {in }} \cdot \boldsymbol{n}=\varepsilon_{\mathrm{d}} \boldsymbol{E}_{\text {out }} \cdot \boldsymbol{n}$ imposed at the metal surface $(\boldsymbol{n}=\boldsymbol{r} / \boldsymbol{r}$ is the normal unit vector directed outward the metal sphere). Thus the internal field $\boldsymbol{E}_{\text {in }}$ is given by Eqs. (7.1), (7.2), (7.4), and (7.5), where the field $\boldsymbol{E}_{0}$ is replaced by $\boldsymbol{E}_{1}$. The pre-factor $B$ in Eq. (7.34) is also found by matching the fields $\boldsymbol{E}_{\text {in }}$ and $\boldsymbol{E}_{\text {out }}$ at the surface of a metal grain; this gives $B=\left(\varepsilon_{\mathrm{d}}-\tilde{\varepsilon}_{\mathrm{m}}\right) /\left(2 \varepsilon_{\mathrm{d}}+\tilde{\varepsilon}_{\mathrm{m}}\right)$ as in Eq. (7.6), where the renormalized metal dielectric constant $\tilde{\varepsilon}_{\mathrm{m}}$ is given by Eq. (7.3).

When the local electric field $\boldsymbol{E}(\boldsymbol{r})$ is known for one lattice cell, the effective dielectric constant of an electromagnetic crystal can be found following the procedure described above. The homogenization procedure can be divided into five steps (I)-(V):

(I) First, the average electric field is calculated as

$$
\langle\boldsymbol{E}\rangle=\frac{1}{V} \int \boldsymbol{E}(\boldsymbol{r}) \mathrm{d} V=\frac{1}{V}\left(\int \boldsymbol{E}_{\mathrm{in}}(\boldsymbol{r}) \mathrm{d} V+\int \boldsymbol{E}_{\text {out }}(\boldsymbol{r}) \mathrm{d} V\right),
$$

where the first integration is over the volume $V=\mathscr{L}^{3}$ of the lattice cell, and the fields $\boldsymbol{E}_{\text {in }}$ and $\boldsymbol{E}_{\text {out }}$ are integrated inside and outside of the metal grain, respectively. Note that in the considered dipole approximation, when $\boldsymbol{E}_{\text {out }}$ has the form of Eq. (7.34), the integration of the dipole term in Eq. (7.34) gives zero, if the crystal possesses inversion symmetry. It will be assumed that this is the case and, therefore, the second integral in Eq. (I) equals $V^{-1} \int \boldsymbol{E}_{\text {out }}(\boldsymbol{r}) \mathrm{d} V=\boldsymbol{E}_{1}(1-p)$, where $p=(4 / 3) \pi a^{3} / V$ is the volume concentration of metal (filling factor).

(II) Second, the eddy magnetic induction, defined as

$$
\boldsymbol{B}_{E}(\boldsymbol{r})=-\mathrm{i} \operatorname{curl}(\boldsymbol{E}(\boldsymbol{r})) / k,
$$

is calculated. In the considered case of a crystal composed from metal spheres, the magnetic induction $\boldsymbol{B}_{E}(\boldsymbol{r})$ is equal to zero outside the metal sphere and is given by Eq. (7.5) for the points $\boldsymbol{r}$ inside the sphere.

(III) Third, the moment $\langle\boldsymbol{L}\rangle=V^{-1} \int \boldsymbol{L} \mathrm{d} V=\mathrm{i} k /(8 \pi V) \int \boldsymbol{r} \times \boldsymbol{B}_{E} \mathrm{~d} V$ of the eddy magnetic induction $\boldsymbol{B}_{E}$ is calculated. Then the average, curl-free local electric field $\boldsymbol{E}_{0}$ from Eq. (7.20) equals

$$
\boldsymbol{E}_{0}=\langle\boldsymbol{E}\rangle-4 \pi\langle\boldsymbol{L}\rangle .
$$

For the considered metal-sphere crystal, the calculated electric field $\boldsymbol{E}_{0}$ equals

$$
\boldsymbol{E}_{0}=\left[p 3 \varepsilon_{\mathrm{d}} /\left(2 \varepsilon_{\mathrm{d}}+\tilde{\varepsilon}_{\mathrm{m}}\right)+(1-p)\right] \boldsymbol{E}_{1},
$$

where $\tilde{\varepsilon}_{\mathrm{m}}$ is given by Eq. (7.3). 
(IV) The average electric displacement is defined as

$$
\langle\boldsymbol{D}\rangle=\frac{1}{V} \int \varepsilon(\boldsymbol{r}) \boldsymbol{E}(\boldsymbol{r}) \mathrm{d} V,
$$

where the local dielectric constant takes values $\varepsilon_{\mathrm{m}}$ and $\varepsilon_{\mathrm{d}}$ inside and outside a metal grain, respectively. For the metal-sphere crystal, the average electric displacement equals

$$
\langle\boldsymbol{D}\rangle=\left[p \frac{3 \varepsilon_{\mathrm{d}} \tilde{\varepsilon}_{\mathrm{m}}}{2 \varepsilon_{\mathrm{d}}+\tilde{\varepsilon}_{\mathrm{m}}}+(1-p) \varepsilon_{\mathrm{d}}\right] \boldsymbol{E}_{1}
$$

where the renormalized metal dielectric constant $\tilde{\varepsilon}_{\mathrm{m}}$ is given by Eq. (7.3).

(V) Finally, the effective dielectric constant is determined from Eq. (7.33) as a coefficient between $\langle\boldsymbol{D}\rangle$ and $\boldsymbol{E}_{0}$, namely, $\langle\boldsymbol{D}\rangle=\varepsilon_{\mathrm{e}} \boldsymbol{E}_{0}$.

Then it follows from Eqs. (7.35) and (7.36) that the effective dielectric constant $\varepsilon_{\mathrm{e}}$ for the metal-sphere crystal equals

$$
\varepsilon_{\mathrm{e}}=\varepsilon_{\mathrm{d}} \frac{(1+2 p) \tilde{\varepsilon}_{\mathrm{m}}+2(1-p) \varepsilon_{\mathrm{d}}}{(1-p) \tilde{\varepsilon}_{\mathrm{m}}+(p+2) \varepsilon_{\mathrm{d}}}
$$

The above consideration (I)-(V) repeated for the magnetic field defines the effective magnetic permeability $\mu_{\mathrm{e}}$ for a cubic crystal composed of metal spheres as follows:

$$
\mu_{\mathrm{e}}=\frac{(1+2 p) \tilde{\mu}_{\mathrm{m}}+2(1-p)}{(1-p) \tilde{\mu}_{\mathrm{m}}+(p+2)},
$$

where $\tilde{\mu}_{\mathrm{m}}$ is the renormalized metal magnetic permeability given by Eq. (7.11); it is still supposed, for simplicity, that neither a dielectric host nor metal spheres have magnetic properties, i.e., $\mu_{\mathrm{d}}=\mu_{\mathrm{m}}=1$.

In the quasistatic case, when the skin effect is negligible, Eqs. (7.37) and (7.38) give the well known Maxwell-Garnett formulae for the effective parameters. It has been demonstrated that the Maxwell-Garnett approximation, which emerges from the dipole approximation, gives very accurate results for the effective properties of various metal-dielectric periodic composites, even at large filling factors $p<0.5[61,131,132]$. Then it is reasonable to conjecture that in a nonquasistatic case Eqs. (7.37) and (7.38) hold for this concentration range. For a large filling factor $p$, the local fields and effective parameters of the electromagnetic crystals can be defined using the Rayleigh technique developed by McPhedran and co-workers [131,132]. Again, provided that the internal field has been found, the effective parameters $\varepsilon_{\mathrm{e}}$ and $\mu_{\mathrm{e}}$ can be calculated from the procedure described by Eqs. (I)-(V).

Consider now a strong skin effect when the absolute value of the wave vector $k_{\mathrm{m}}$ in metal particles tends to infinity, i.e., $\left|k_{\mathrm{m}}\right| a \rightarrow \infty$. Let us also suppose that $\operatorname{Im}\left(k_{\mathrm{m}}\right) a \rightarrow+\infty$, that is the electric and magnetic fields fall exponentially in a metal grain, being confined mainly to the skin depth $\delta=1 / \operatorname{Im}\left(k_{\mathrm{m}}\right)$. Recall that for the positive values of the metal conductivity $\sigma_{\mathrm{m}}$ (which is typical for most metals for the radio, microwave and far-infrared frequencies $\omega$ ) the skin depth $\delta$ is equal to $\delta=c / \sqrt{2 \pi \sigma_{\mathrm{m}} \omega}$ and the wave vector $k_{\mathrm{m}}=(1+\mathrm{i}) / \delta$. Then as follows from Eqs. (7.3) and (7.11) the renormalized metal dielectric constant $\tilde{\varepsilon}_{\mathrm{m}}$ and the magnetic permeability $\tilde{\mu}_{\mathrm{m}}$ are approximated as

$$
\tilde{\varepsilon}_{\mathrm{m}} \approx 2 \mathrm{i} k_{\mathrm{m}} a /(k a)^{2}
$$


and

$$
\tilde{\mu}_{\mathrm{m}} \approx 2 \mathrm{i} /\left(k_{\mathrm{m}} a\right)
$$

from which it follows that $\left|\tilde{\varepsilon}_{\mathrm{m}}\right| \gg 1$ and $\left|\tilde{\mu}_{\mathrm{m}}\right| \ll 1$ when $\left|k_{\mathrm{m}}\right| a \sim a / \delta \gg 1$. Substitution of these estimates in Eqs. (7.37) and (7.38) gives

$$
\varepsilon_{\mathrm{e}}=\varepsilon_{\mathrm{d}}(1+2 p) /(1-p),
$$

and

$$
\mu_{\mathrm{e}}=2(1-p) /(p+2),
$$

for the effective parameters of the metal-sphere crystal in the case of strong skin effect. Thus obtained $\varepsilon_{\mathrm{e}}$ and $\mu_{\mathrm{e}}$ do not depend on the metal properties at all. The effective refractive index $n=\sqrt{\varepsilon_{\mathrm{e}} \mu_{\mathrm{e}}}=\sqrt{\varepsilon_{\mathrm{d}} 2(1+2 p) /(p+2)}$ is of the order of one for almost all filling factors. The effective surface impedance $\zeta=\sqrt{\mu_{\mathrm{e}} / \varepsilon_{\mathrm{e}}}$ determines reflection at the interface of a system, e.g., the normal reflection at the interface with a vacuum equals $R=(\zeta-1) /(\zeta+1)$. It follows from Eqs. (7.41) and (7.42) that the effective surface impedance $\zeta$ can be estimated as

$$
\zeta=(1-p) \sqrt{\frac{2}{\varepsilon_{\mathrm{d}}(1+2 p)(p+2)}},
$$

and it is also independent of the metal properties and almost linearly decreases with increasing the filling factor $p$. The reflection coefficient is given by

$$
R \simeq(n-1) /(n+1)+9 p n / 2(1+n)^{2},
$$

where $n=\sqrt{\varepsilon_{\mathrm{d}}}$ is the refractive index for a dielectric host. The reflection coefficient $R$ is real and it increases almost linearly up to the value of the filling factor $p_{c} \approx 0.524$ corresponding to the close-packed metal spheres in the cubic lattice.

It follows from Eqs. (7.41)-(7.114) that losses in the crystal are negligible. Electromagnetic field slips without loss between the metal grains and the electromagnetic crystal is essentially transparent. Note that the dipole approximation used for obtaining Eqs. (7.41)-(7.114) does not hold when the filling factor $p$ approaches the close-packing limit $p_{c}$ since the direct contact between the metal spheres becomes important. When the spheres are in contact, the crystal properties are close to those in mesh wire electromagnetic crystals (see below). The distance $b$ between the spheres, when this crossover takes place, can be estimated by equating the absolute values of the effective conductance of a metal sphere $\left|\Sigma_{\mathrm{m}}\right| \sim\left|\tilde{\varepsilon}_{\mathrm{m}}\right| \omega a$ and the capacitive conductance $\left|\Sigma_{\mathrm{c}}\right| \sim \varepsilon_{\mathrm{d}} \omega a^{2} / b$ between the metal grains (where the renormalized metal dielectric constant is given by Eq. (7.39), for the strong skin effect). Thus the estimate $(b / a)<\varepsilon_{\mathrm{d}} k a / \sqrt{\left|\varepsilon_{\mathrm{m}}\right|} \ll 1$ could be suggested for the proximity $b$ between the metal spheres when Eq. (7.114) is violated and an electromagnetic crystal becomes opaque.

\subsubsection{Wire mesh electromagnetic crystal}

We consider now electromagnetic properties of three-dimensional metal wire mesh configurated in a cubic lattice. This electromagnetic crystal can be thought of as an opposite limit to the above 
considered case of unconnected metal spheres. It is worthwhile to consider first a two-dimensional array of metal cylinders assembled in a square lattice with period $\mathscr{L}$. The direction of the incident electromagnetic wave is supposed to be perpendicular to the cylinders. Then there are two basic polarizations TE (transverse electric) and TM (transverse magnetic) polarizations. For TE polarization, considered first, the electric field of an incident wave is perpendicular to the direction of the cylinders. The problem of the local electric field in a lattice cell with a metal cylinder in the center is a two-dimensional analog of the electric field distribution considered above for a cell of metalsphere crystals. Therefore, the following expression can be written in the dipole approximation for the electric field outside the cylinder [cf. Eqs. (7.34)]

$$
\boldsymbol{E}_{\mathrm{out}}(\boldsymbol{r})=\boldsymbol{E}_{1}+B a^{2} \nabla\left(\frac{\boldsymbol{E}_{1} \cdot \boldsymbol{r}}{r^{2}}\right)
$$

where $\boldsymbol{E}_{1}$ is a vector aligned with the electric field of the incident wave, $B$ is a numerical coefficient, $\boldsymbol{r}$ is a two-dimensional vector in the plane perpendicular to the cylinders. The electric field inside the cylinder $\boldsymbol{E}_{\text {in }}$, which matches the outside field $\boldsymbol{E}_{\text {out }}$ given by Eq. (7.45), can be written as [82, Section 59]:

$$
\boldsymbol{E}_{\text {in }}(\boldsymbol{r})=A \operatorname{curl} \operatorname{curl}\left[J_{0}\left(k_{\mathrm{m}} r\right) \boldsymbol{E}_{1}\right],
$$

where $A$ is another coefficient and $J_{0}$ is the Bessel function of the zeroth order. As in the case of a sphere, the coefficients $A$ and $B$ are found from the boundary conditions $\boldsymbol{E}_{\text {in }} \times \boldsymbol{n}=\boldsymbol{E}_{\text {out }} \times \boldsymbol{n}$ and $\varepsilon_{\mathrm{m}} \boldsymbol{E}_{\mathrm{in}} \cdot \boldsymbol{n}=\varepsilon_{\mathrm{d}} \boldsymbol{E}_{\text {out }} \cdot \boldsymbol{n}$ imposed at the surface of the cylinder, where $\boldsymbol{n}=\boldsymbol{r} / \boldsymbol{r}$ is a unit normal vector directed outward the metal cylinder, namely

$$
B=\frac{\varepsilon_{\mathrm{d}}-\tilde{\varepsilon}_{\mathrm{m}}}{\varepsilon_{\mathrm{d}}+\tilde{\varepsilon}_{\mathrm{m}}}
$$

and

$$
A=\frac{2 \varepsilon_{\mathrm{d}}}{\left(\varepsilon_{\mathrm{d}}+\tilde{\varepsilon}_{\mathrm{m}}\right) k_{\mathrm{m}}^{2} J_{0}\left(k_{\mathrm{m}} a\right)\left[1-F\left(k_{\mathrm{m}} a\right)\right]},
$$

where the renormalized metal dielectric constant $\tilde{\varepsilon}_{\mathrm{m}}$ and function $F_{1}$ are given by

$$
\tilde{\varepsilon}_{\mathrm{m}}=\varepsilon_{\mathrm{m}} F_{1}\left(k_{\mathrm{m}} a\right) /\left[1-F_{1}\left(k_{\mathrm{m}} a\right)\right],
$$

and

$$
F_{1}(x)=J_{1}(x) / x J_{0}(x),
$$

respectively, with $J_{1}$ being the first-order Bessel function. Given the electric field in the lattice cell, the effective dielectric constant of the wire mesh crystal can be found from the procedure (I)-(V) outlined above:

(I) The electric field averaged over the cell is equal to

$$
\langle\boldsymbol{E}\rangle=\frac{1}{\mathscr{L}^{2}}\left(\int_{\boldsymbol{r}<a} \boldsymbol{E}_{\mathrm{in}} \mathrm{d} \boldsymbol{r}+\int_{\boldsymbol{r}>a} \boldsymbol{E}_{\text {out }} \mathrm{d} \boldsymbol{r}\right)=\boldsymbol{E}_{1}\left[\frac{2 p \varepsilon_{\mathrm{d}} F\left(k_{\mathrm{m}} a\right)}{\left(\varepsilon_{\mathrm{d}}+\tilde{\varepsilon}_{\mathrm{m}}\right)\left[1-F\left(k_{\mathrm{m}} a\right)\right]}+(1-p)\right],
$$

where $p=\pi a^{2} / \mathscr{L}^{2}$ is the filling factor. 
(II) The magnetic induction generated by the electric field inside the metal cylinder is as follows:

$$
\boldsymbol{B}_{E}(\boldsymbol{r})=\left\{0,0,-E_{1} \frac{2 \mathrm{i} \varepsilon_{\mathrm{d}} k_{\mathrm{m}} r F\left(k_{\mathrm{m}} r\right) J_{0}\left(k_{\mathrm{m}} r\right)}{\left(\varepsilon_{\mathrm{d}}+\tilde{\varepsilon}_{\mathrm{m}}\right)\left[1-F\left(k_{\mathrm{m}} a\right)\right] J_{0}\left(k_{\mathrm{m}} a\right)} \sin (\phi)\right\},
$$

where $\phi$ is a polar angle, so that the vector $\boldsymbol{r}$ equals $\boldsymbol{r}=\{r \cos \phi, r \sin \phi, 0\}$.

(III) The average curl-free local field is given by

$$
\boldsymbol{E}_{0}=\langle\boldsymbol{E}\rangle-\frac{\mathrm{i} k}{\mathscr{L}^{2}} \int_{\boldsymbol{r}<a} \boldsymbol{r} \times \boldsymbol{B}_{E}(\boldsymbol{r}) \mathrm{d} \boldsymbol{r}=\boldsymbol{E}_{1}\left[\frac{2 p \varepsilon_{\mathrm{d}}}{\left(\varepsilon_{\mathrm{d}}+\tilde{\varepsilon}_{\mathrm{m}}\right)}+(1-p)\right] .
$$

(IV) The average electric displacement is as follows:

$$
\langle\boldsymbol{D}\rangle=\frac{1}{\mathscr{L}^{2}}\left(\int_{\boldsymbol{r}<a} \varepsilon_{\mathrm{m}} \boldsymbol{E}_{\mathrm{in}} \mathrm{d} \boldsymbol{r}+\int_{\boldsymbol{r}>a} \varepsilon_{\mathrm{d}} \boldsymbol{E}_{\mathrm{out}} \mathrm{d} \boldsymbol{r}\right)=\boldsymbol{E}_{1}\left[\frac{2 p \varepsilon_{\mathrm{d}} \tilde{\varepsilon}_{\mathrm{m}}}{\left(\varepsilon_{\mathrm{d}}+\tilde{\varepsilon}_{\mathrm{m}}\right)}+(1-p) \varepsilon_{\mathrm{d}}\right] .
$$

Finally, the effective dielectric constant of the mesh crystal is given by

$$
\varepsilon_{\mathrm{e} \perp}=\frac{|\langle\boldsymbol{D}\rangle|}{\left|\boldsymbol{E}_{0}\right|}=\varepsilon_{\mathrm{d}} \frac{2 p \tilde{\varepsilon}_{\mathrm{m}}+(1-p)\left(\varepsilon_{\mathrm{d}}+\tilde{\varepsilon}_{\mathrm{m}}\right)}{2 p \varepsilon_{\mathrm{d}}+(1-p)\left(\varepsilon_{\mathrm{d}}+\tilde{\varepsilon}_{\mathrm{m}}\right)} .
$$

Now recall that this result holds for the TE polarization, when the electric field of an incident wave is perpendicular to the cylinder axes. Therefore, the effective dielectric constant is denoted as $\varepsilon_{\mathrm{e} \perp}$. In the case of strong skin effect the renormalized metal permittivity is large, $\left|\tilde{\varepsilon}_{\mathrm{m}}\right| \gg 1$ [see Eq. (7.39)]; then the effective dielectric constant takes the following asymptotic form:

$$
\varepsilon_{\mathrm{e} \perp}=\varepsilon_{\mathrm{d}}(1+p) /(1-p),
$$

which is independent of the metal properties [cf. Eq. (7.41)].

Now the effective magnetic properties are considered. It is still assumed that the metal and dielectric components are nonmagnetic, i.e. $\mu_{\mathrm{m}}=\mu_{\mathrm{d}}=1$. For the TE polarization the magnetic field is parallel to the cylinders. The local magnetic field outside the cylinder is uniform and is denoted as $\boldsymbol{H}_{1}$. The field inside the cylinder is equal to $\boldsymbol{H}_{1} J_{0}\left(k_{\mathrm{m}} r\right) / J_{0}\left(k_{\mathrm{m}} a\right)$, where $J_{0}$ is the Bessel function of the zeroth order. Again, the procedure (I)-(V) allows to find the effective magnetic permeability.

(I) The average magnetic field is given by

$$
\langle\boldsymbol{H}\rangle=\boldsymbol{H}_{1}\left[2 p F_{1}\left(k_{\mathrm{m}} a\right)+(1-p)\right],
$$

where the function $F$ is defined in Eq. (7.50).

(II) The "Foucault" electric displacement $\boldsymbol{D}_{H}$ is circular, i.e., in the cylindrical coordinates it has only the " $\phi$ " component

$$
D_{H, \phi}(r)=\mathrm{i} \frac{k_{\mathrm{m}} J_{1}\left(k_{\mathrm{m}} r\right)}{k J_{0}\left(k_{\mathrm{m}} a\right)} H_{1}
$$

(III) The average curl-free magnetic field $\boldsymbol{H}_{0}$ is given by $\boldsymbol{H}_{1}$. 
(IV) Since neither metal nor dielectric host possesses intrinsic magnetism, the average magnetic induction $\langle\boldsymbol{B}\rangle$ coincides with the average magnetic field $\langle\boldsymbol{H}\rangle$ given by Eq. (7.57), and (V) the effective magnetic permeability $\mu_{\mathrm{e} \|}=|\langle\boldsymbol{B}\rangle| /\left|\boldsymbol{H}_{0}\right|$ is as follows:

$$
\mu_{\mathrm{e} \|}=2 p F\left(k_{\mathrm{m}} a\right)+(1-p) .
$$

Since for the considered TE polarization of the magnetic field in the incident wave is parallel to the axes of the cylinders the effective permeability is denoted as $\mu_{\mathrm{e} \|}$. In the limit of strong skin effect $\left(a \operatorname{Im} k_{\mathrm{m}} \rightarrow+\infty\right)$, the effective permeability $\mu_{\mathrm{e} \|}$ is given by

$$
\mu_{\mathrm{e} \|}=(1-p) \text {. }
$$

In the same limit, the effective refractive index $n_{\mathrm{e}}=\sqrt{\varepsilon_{\mathrm{e} \perp} \mu_{\mathrm{e} \|}}$ takes the following form:

$$
n_{\mathrm{e}}=\sqrt{\varepsilon_{\mathrm{d}}(1+p)} .
$$

This result for $n_{\mathrm{e}}$ coincides with the effective refractive index obtained in Ref. [131] from quite different considerations. We would like to stress out that the difference between the refractive index $n_{\mathrm{e}}$ and the value $\sqrt{\varepsilon_{\mathrm{e} \perp}}$ discussed in Ref. [131], arises naturally in the discussed approach as a result of the effective magnetic properties of electromagnetic crystals. Thus the ratio $n_{\mathrm{e}} / \sqrt{\varepsilon_{\mathrm{e} \perp}}=\sqrt{\mu_{\mathrm{e} \|}}$ is not equal to one.

The most interesting results are obtained for the TM polarization, where the electric field of the incident wave is parallel to the cylinders while the magnetic field is perpendicular. In the long-wavelength limit considered throughout the paper (when the wavelength $\lambda$ is much larger than the size $\mathscr{L}$ of the lattice cell) the local electric field in the cell can be found as above in the dipole approximation. In this approximation the local TM electric field has a circular symmetry. Then the electric field in the cell is obtained from the Maxwell's equations as follows (recall that a square cell is considered):

$$
\begin{aligned}
& \boldsymbol{E}(\boldsymbol{r})=\boldsymbol{E}_{1} J_{0}\left(k_{\mathrm{m}} r\right), \quad r<a \\
& \boldsymbol{E}(\boldsymbol{r})=\boldsymbol{E}_{1}\left[J_{0}\left(k_{\mathrm{m}} a\right)-k_{\mathrm{m}} a J_{1}\left(k_{\mathrm{m}} a\right) \log \left(\frac{r}{a}\right)\right], \quad r>a \&|y|<\mathscr{L} / 2 \&|x|<\mathscr{L} / 2 ;
\end{aligned}
$$

where $\boldsymbol{r}=\{x, y\}$ is a two-dimensional vector in the plane perpendicular to the cylinder and $\boldsymbol{E}_{1}=\left\{0,0, E_{1}\right\}$ is a vector aligned with the cylinder axis and proportional to the amplitude of the electric field in the incident wave.

The homogenization procedure (I)-(V) allows to find the effective dielectric constant $\varepsilon_{\mathrm{e}||}$ for the TM polarization. (I) The average electric field is given by

$$
\langle\boldsymbol{E}\rangle=\boldsymbol{E}_{1}\left\{(1-p) J_{0}\left(k_{\mathrm{m}} a\right)+\frac{J_{1}\left(k_{\mathrm{m}} a\right)}{4 a k_{\mathrm{m}}}\left[8 p-\left(a k_{\mathrm{m}}\right)^{2}(2 p-6+\pi)+2\left(a k_{\mathrm{m}}\right)^{2} \log \left(\frac{2 a^{2}}{\mathscr{L}^{2}}\right)\right]\right\} .
$$

(II) The circular magnetic induction $\boldsymbol{B}_{E}$ generated by the local electric field has only the " $\phi$ " component in the cylindrical coordinates:

$$
\begin{aligned}
& B_{E, \phi}(\boldsymbol{r})=-\mathrm{i} E_{1} k_{\mathrm{m}} J_{1}\left(k_{\mathrm{m}} r\right) / k, \quad r<a, \\
& B_{E, \phi}(\boldsymbol{r})=-\mathrm{i} E_{1} k_{\mathrm{m}} a J_{1}\left(k_{\mathrm{m}} a\right) /(k r), \quad r>a \&|y|<\mathscr{L} / 2 \&|x|<\mathscr{L} / 2 .
\end{aligned}
$$


(III) The average curl-free electric field $\boldsymbol{E}_{0}$ is as follows:

$$
\boldsymbol{E}_{0}=\boldsymbol{E}_{1}\left\{J_{0}\left(k_{\mathrm{m}} a\right)+\frac{1}{4} k_{\mathrm{m}} a J_{1}\left(k_{\mathrm{m}} a\right)\left[4-\pi+2 \log \left(\frac{2 a^{2}}{\mathscr{L}^{2}}\right)\right]\right\} .
$$

(iv) The electric displacement averaged over the cell is given by

$$
\langle\boldsymbol{D}\rangle=\boldsymbol{E}_{1}\left\{\varepsilon_{\mathrm{d}}(1-p) J_{0}\left(k_{\mathrm{m}} a\right)+J_{1}\left(k_{\mathrm{m}} a\right)\left[\frac{2 k_{\mathrm{m}} p}{a k^{2}}-\frac{\varepsilon_{\mathrm{d}} k_{\mathrm{m}} a}{4}\left(2 p-6+\pi-2 \log \left(\frac{2 a^{2}}{\mathscr{L}^{2}}\right)\right)\right]\right\},
$$

and (V) the effective dielectric constant $\varepsilon_{\mathrm{e} \|}=|\langle\boldsymbol{D}\rangle| /\left|\boldsymbol{E}_{0}\right|$ is as follows:

$$
\varepsilon_{\mathrm{e}}=\frac{4 \varepsilon_{\mathrm{d}}(1-p)+\varepsilon_{\mathrm{m}} F_{1}\left(k_{\mathrm{m}} a\right)\left\{8 p+(a k)^{2} \varepsilon_{\mathrm{d}}\left[6-2 p-\pi+2 \log \left(\frac{2 a^{2}}{\mathscr{L}^{2}}\right)\right]\right\}}{4+\left(k_{\mathrm{m}} a\right)^{2} F_{1}\left(k_{\mathrm{m}} a\right)\left[4-\pi+2 \log \left(\frac{2 a^{2}}{\mathscr{L}^{2}}\right)\right]}
$$

where the function $F_{1}$ is defined in Eq. (7.50). For the important case of thin cylinders, when the radius $a$ of the cylinders is much less than the lattice period $\mathscr{L}$ [i.e. when $\log (\mathscr{L} / a) \gg 1$ ], the above expression can be simplified as follows:

$$
\varepsilon_{\mathrm{e} \|}=\varepsilon_{\mathrm{d}}-p \frac{2 \varepsilon_{\mathrm{m}} F_{1}\left(k_{\mathrm{m}} a\right)}{\left(a k_{\mathrm{m}}\right)^{2} F_{1}\left(k_{\mathrm{m}} a\right) \log \left(\frac{\mathscr{L}}{a}\right)-1} .
$$

This equation in turn can be rewritten as $\varepsilon_{\mathrm{e} \|}=\varepsilon_{\mathrm{d}}+\tilde{\varepsilon}_{\mathrm{m}} p$, where $\tilde{\varepsilon}_{\mathrm{m}}$ is the renormalized metal dielectric constant for the TM polarization. It is interesting to note that this renormalized metal dielectric constant $\tilde{\varepsilon}_{\mathrm{m}}$ coincides with the renormalized dielectric constant for the conducting-stick composites considered in Ref. [107].

In the case of strong skin effect when $a \operatorname{Im} k_{\mathrm{m}} \rightarrow+\infty$, Eq. (7.68) simplifies further to the expression

$$
\varepsilon_{\mathrm{e}||}=\varepsilon_{\mathrm{d}}-\frac{2 p}{(a k)^{2} \log \left(\frac{\mathscr{L}}{a}\right)}=\varepsilon_{\mathrm{d}}-\frac{\omega^{2}}{\omega_{\mathrm{p}}^{2}}
$$

that does not depend on the metal conductivity. Here the "plasma frequency" is given by

$$
\omega_{\mathrm{p}}^{2}=\frac{2 c^{2} p}{a^{2} \log \left(\frac{\mathscr{L}}{a}\right)}=\frac{2 \pi c^{2}}{\mathscr{L}^{2} \log \left(\frac{\mathscr{L}}{a}\right)} .
$$

It follows from Eq. (7.69) that the effective dielectric constant becomes negative for the frequency $\omega$ smaller than the renormalized plasma frequency $\tilde{\omega}_{\mathrm{p}}=\omega_{\mathrm{p}} / \sqrt{\varepsilon_{\mathrm{d}}}$. Therefore, the incident TM 
electromagnetic wave decays exponentially in the electromagnetic crystal for $\omega<\tilde{\omega}_{\mathrm{p}}$. The negative values of the effective dielectric constant in metal-dielectric composites containing conducting sticks were predicted theoretically [107,133] and obtained experimentally [134,135]. The cutoff frequency in Eq. (7.70) was first suggested in [63], where it was related to the effective plasma frequency of the electron gas in an electromagnetic crystal.

The problem of the effective magnetic permeability $\mu_{\mathrm{e} \perp}$ for the TM polarization, when the magnetic field of the incident wave is perpendicular to the axis of the cylinders, coincides with the problem of the effective dielectric constant $\varepsilon_{\mathrm{e} \perp}$ for the TE polarization. Thus the distribution of the local magnetic field in the lattice cell can be found from Eq. (7.45)-(7.50) by the replacement $\boldsymbol{H}(\boldsymbol{r}) \rightarrow \boldsymbol{E}(\boldsymbol{r}), \tilde{\varepsilon}_{\mathrm{m}} \rightarrow \tilde{\mu}_{\mathrm{m}}, \varepsilon_{\mathrm{m}} \rightarrow 1$ and $\varepsilon_{\mathrm{d}} \rightarrow 1$. The effective magnetic permeability $\mu_{\mathrm{e} \perp}$ can also be obtained from $\varepsilon_{\mathrm{e} \perp}$ by using the above replacement

$$
\mu_{\mathrm{e} \perp}=\frac{2 p \tilde{\mu}_{\mathrm{m}}+(1-p)\left(1+\tilde{\mu}_{\mathrm{m}}\right)}{2 p+(1-p)\left(1+\tilde{\mu}_{\mathrm{m}}\right)}
$$

where it is assumed again that neither metal nor dielectric possess intrinsic magnetism.

Consider now a three-dimensional conducting wire mesh configurated in the cubic lattice. Let us suppose, for simplicity, that the wires are sufficiently thin and, therefore, the filling factor $p$ is small. Then the effect of the intersections of the wires can be neglected since these effects give corrections of the order of $p^{2}$. Thus the following expression are obtained for the effective dielectric constant $\varepsilon_{\mathrm{e}}=\varepsilon_{\mathrm{e} \|}+2 \varepsilon_{\mathrm{e} \perp}-2 \varepsilon_{\mathrm{d}}$ and for the magnetic permeability $\mu_{\mathrm{e}}=\mu_{\mathrm{e} \|}+2 \mu_{\mathrm{e} \perp}-2$. The quantities $\varepsilon_{\mathrm{e} \|}, \varepsilon_{\mathrm{e} \perp}, \mu_{\mathrm{e} \|}$ and $\mu_{\mathrm{e} \perp}$ are given by Eqs. (7.67), (7.55), (7.59), and (7.71) respectively, where the filling factor should be changed from $p$ to $p / 3$, which corresponds to three metal cylinders in a lattice cell.

Below it is supposed that the metal conductivity is real (i.e., the dielectric constant is purely imaginary) and, therefore, there is no internal resonances in metal wires. This situation is typical for simple nonstructured wires for the frequencies up to the far-infrared. We will not consider here the plasmon resonance in a metal wire, which occurs in the optical spectral range when $\varepsilon_{\mathrm{m}}^{\prime}=-\varepsilon_{\mathrm{d}}$. In the absence of the resonances and at the filling factor $p \ll 1$, the effective dielectric constant is approximated as $\varepsilon_{\mathrm{e}} \simeq \varepsilon_{\mathrm{e} \| \mid}$ and the magnetic permeability as $\mu_{\mathrm{e}} \simeq 1$. The dependence of the real $\varepsilon_{\mathrm{e}}^{\prime}(\lambda)$ and the imaginary $\varepsilon_{\mathrm{e}}^{\prime \prime}(\lambda)$ parts of the effective dielectric constant on the wavelength $\lambda$ is shown in Figs. 21 and 22, respectively. The behavior of $\varepsilon_{\mathrm{e}}(\lambda)$ changes dramatically when the ratio of the skin depth $\delta$ and the wire radius $a$ decrease. The losses are very significant when $\delta / a>0.1$. Under this condition the imaginary part $\varepsilon_{\mathrm{e}}^{\prime \prime}$ of the effective dielectric constant is larger than the real part, $\varepsilon_{\mathrm{e}}^{\prime \prime}>\varepsilon_{\mathrm{e}}^{\prime}$. On the other hand, the effective dielectric constant $\varepsilon_{\mathrm{e}}$ is almost real when the skin effect is strong. It becomes large in magnitude and negative in sign with decreasing the frequency below the cutoff frequency $\tilde{\omega}_{\mathrm{p}}$. This means that the crystal reflects completely incident em waves, while losses are negligible.

To understand the physical meaning of the negative dielectric constant, let us take into account the fact that the electric field of an incident wave excites not only the current in metal wires but also the circular magnetic induction $\boldsymbol{B}_{E}$ around the wires as it is sketched in Fig. 23 [see also Eq. (7.64)]. Thus, the energy of the incident wave reversibly converts to the energy of the circular magnetic field that concentrates around the wires. This field in turn generates an electric field, which is phaseshifted by $\pi$ with respect to the external field. When this secondary field is larger than the primary 

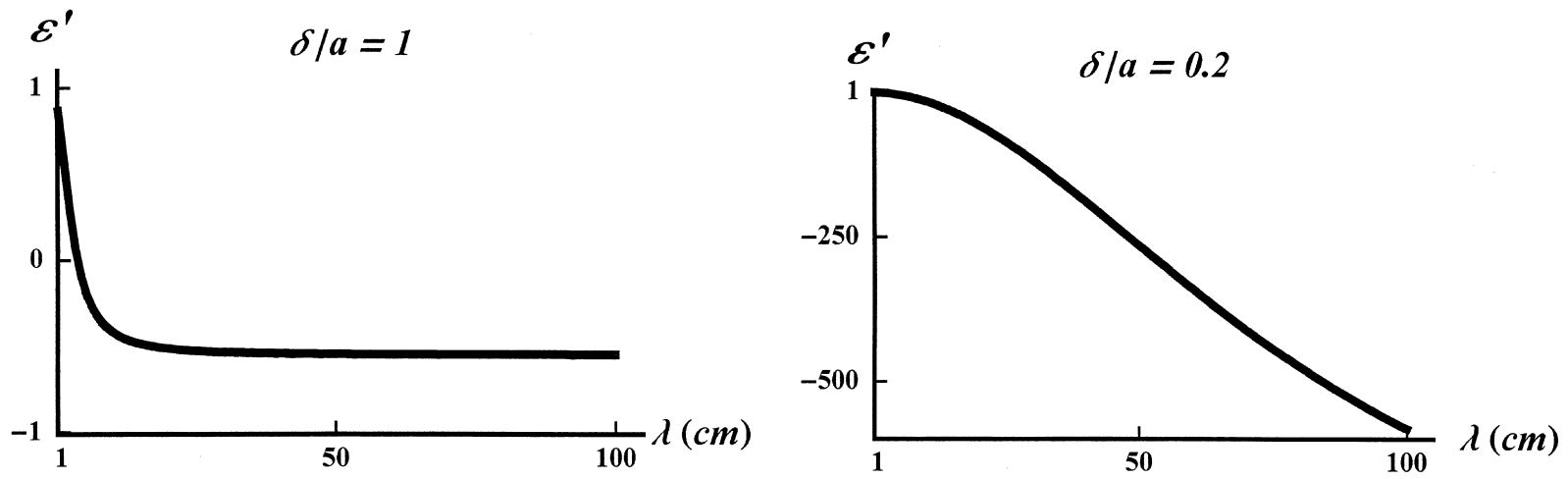

(a)

(b)

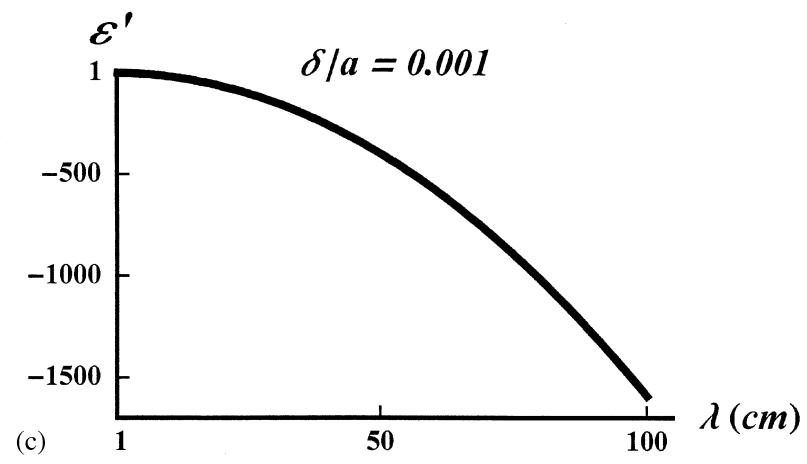

Fig. 21. The real part of the effective dielectric constant $\varepsilon_{\mathrm{e}}^{\prime}(\lambda)$ in the cubic lattice of metal wires. The period of the lattice $L$ equals $L=1 \mathrm{~cm}$, the diameter of the wire $2 a=1 \mathrm{~mm}$. Figs. a-c correspond to different magnitudes of the skin effect at wavelength $\lambda=1 \mathrm{~cm}$.

electric field (which occurs at the strong skin effect) the average electric field is opposite to the external field, so that the effective dielectric constant is negative.

The above equations describe the "macroscopic" electromagnetism in metal-dielectric media. The equations hold on scales much larger than the spatial scale of inhomogeneity, e.g., the size of a metal grain. In the derivation of the macroscopic equations the Foucault currents are taken into account that are excited in metal grains or wires by the high-frequency (HF) magnetic field and the eddy currents of the magnetic induction induced by the HF electric field. The latter has no analogy in the classical electrodynamics since an electric field does not generate the magnetic induction in atoms and molecules. The theory gives macroscopic Maxwell equations describing the wave propagation in metal-dielectric media that include the effective dielectric constant and magnetic permeability. The theory also provides the unambiguous procedure for calculation of the effective parameters.

In the case of periodic metal-dielectric structures, known as electromagnetic crystals, the explicit equations are obtained for the effective dielectric constant and magnetic permeability. Thus the cubic lattice of thin conducting wires appears to have a negative dielectric constant and negligible losses, when the skin effect is strong. The negative values of the dielectric constant result from the eddy currents of the magnetic induction induced by the high-frequency electric field inside and around the metal wires. Such electromagnetic crystals have properties that are similar to bulk 

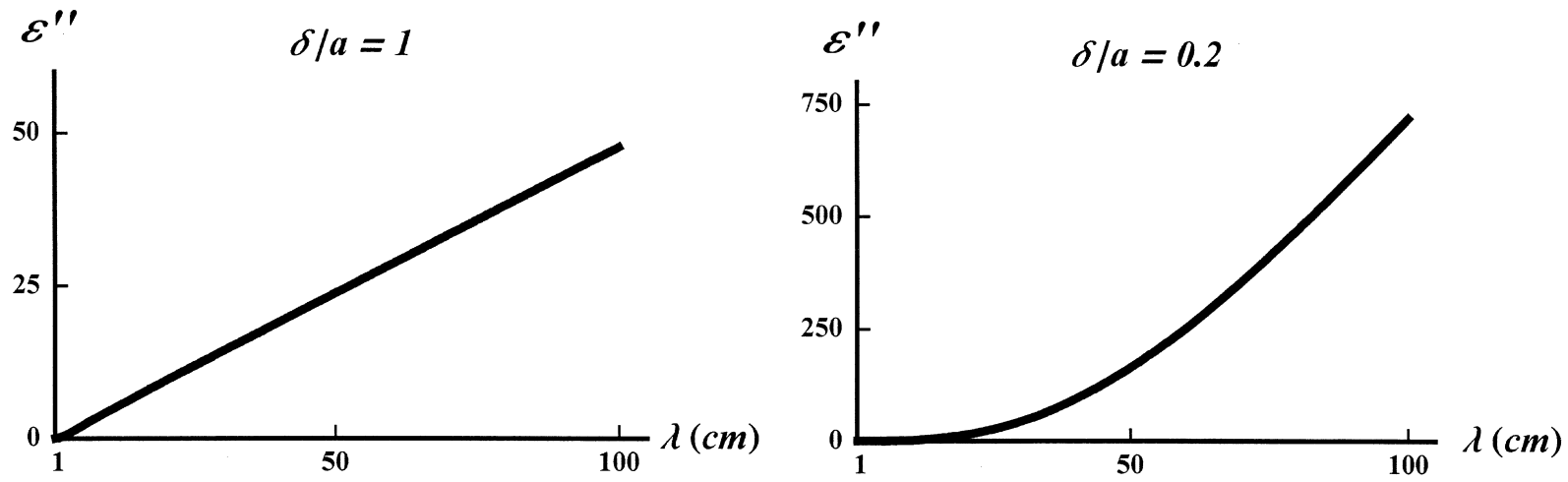

(a)

(b)

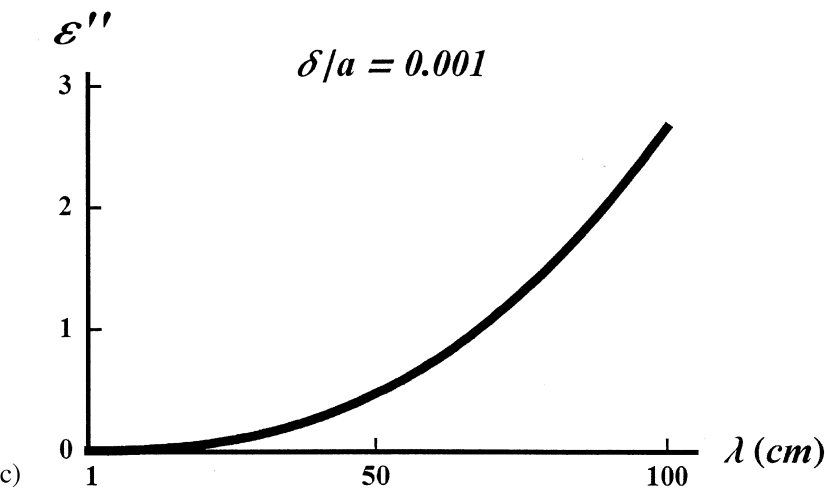

Fig. 22. The imaginary part of the effective dielectric constant $\varepsilon_{\mathrm{e}}^{\prime \prime}(\lambda)$ in the cubic lattice of metal wires. The period of the lattice $L$ is given by $L=1 \mathrm{~cm}$, the wire diameter is $2 a=1 \mathrm{~mm}$. Figs. a-c correspond to different skin effects at wavelength $\lambda=1 \mathrm{~cm}$.

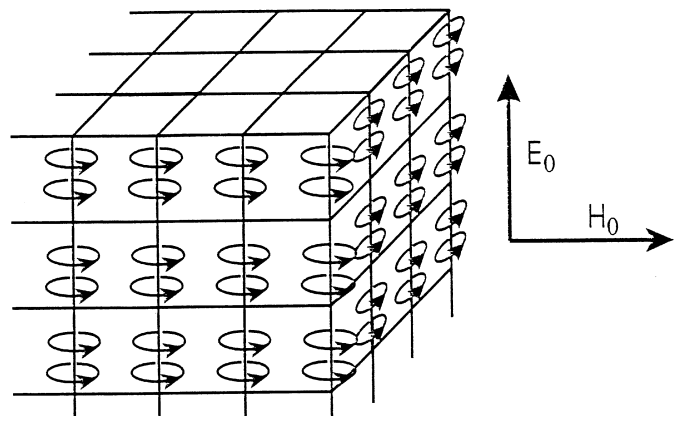

Fig. 23. Circular magnetic field exited in the cubic wire lattice by external electric field.

metal in the optical and near-infrared spectral ranges, e.g., surface plasmons can be excited at the boundaries and also at defects inside a crystal. Another interesting high-frequency property of electromagnetic crystals is the effective magnetism that can be observed, even in systems where neither metal nor dielectric possesses inherent magnetism. 


\subsection{Optical properties of metal semicontinuous films beyond the quasistatic approximation}

The formalism developed in Sections 2-4 cannot be used to describe the optical properties of semicontinuous films in the important case of a strong skin effect in the metal grains. In an attempt to expand the theoretical treatment beyond the quasistatic approximation, an approach has recently been proposed that is based on the full set of Maxwell's equations [39,96,97]. This approach does not use the quasistatic approximation because the fields are not assumed to be curl free inside the physical film. Although that theory was proposed with metal-insulator thin films in mind, it is in fact quite general and can be applied to any kind of inhomogeneous film under appropriate conditions. For reason that will be explained below the above theory is called the "generalized Ohm's law". We present here this new theory.

Below we restrict ourselves to the case where all the external fields are parallel to the plane of the film. This means that an incident wave, as well as the reflected and transmitted waves, are traveling in the direction perpendicular to the film plane. The consideration is focused on the electric and magnetic field magnitudes at certain distances away from the film and relate them to the currents inside the film. We assume that inhomogeneities on a film are much smaller in size than the wavelength $\lambda$ (but not necessarily smaller than the skin depth), so that the fields away from the film are curl-free and can be expressed as gradients of potential fields. The electric and magnetic induction currents averaged over the film thickness obey the usual two-dimensional continuity equations. Therefore the equations for the fields (e.g., $\nabla \times \boldsymbol{E}=0$ ) and the equations for the currents (e.g., $\nabla \cdot \boldsymbol{j}=0$ ) are the same as in the quasistatic case. The only difference is that the fields and the averaged currents are now related by new constitutive equations and that there are magnetic currents as well as electric currents.

To determine these new constitutive equations, we find the electric and magnetic field distributions inside the conductive and dielectric regions of the film. The boundary conditions completely determine solutions of Maxwell's equations for the fields inside a grain when the frequency is fixed. Therefore, the internal fields, which change very rapidly with position in the direction perpendicular to the film, depend linearly on the electric and magnetic field away from the film. The currents inside the film are linear functions of the local internal fields given by the usual local constitutive equations. Therefore, the currents flowing inside the film also depend linearly on the electric and magnetic fields outside the film. However, the electric current averaged over the film thickness now depends not only on the external electric field, but also on the external magnetic field. The same is true for the average magnetic induction current. Thus we have two linear equations that connect the two types of the average internal currents to the external fields. These equations can be considered as generalization of the Ohm's law to the nonquasistatic case and they are denoted as generalized Ohm's law (GOL) [97]. The GOL forms the basis of a new approach to calculating the electromagnetic properties of inhomogeneous films.

\subsubsection{Generalized Ohm's law (GOL) and basic equations}

We base the below consideration on the results of Refs. [39,96,97]. In contrast to the traditional consideration, it is not assumed that the electric and magnetic fields inside a semicontinuous metal film are curl-free and $z$-independent, where the $z$ coordinate is perpendicular to the film plane.

Let us consider first a homogeneous conducting film with a uniform conductivity $\sigma_{\mathrm{m}}$ and thickness $d$ and assume constant values of the electric field $\boldsymbol{E}_{1}$ and magnetic field $\boldsymbol{H}_{1}$ at some 


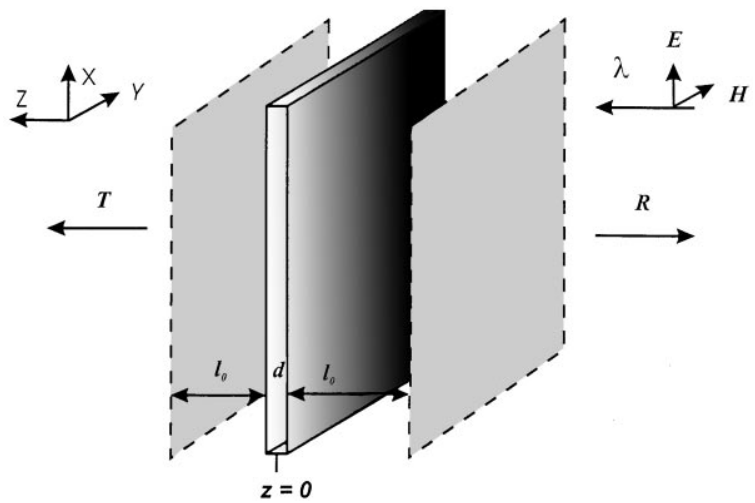

Fig. 24. The scheme used in a theoretical model. Electromagnetic wave of wavelength $\lambda$ is incident on a thin metal-insulator film with thickness $d$. It is partially reflected and absorbed, and the remainder is transmitted through the film.

reference plane $z=-d / 2-l_{0}$ behind the film, as shown in Fig. 24. Under these conditions the fields depend only on the $z$-coordinate, and Maxwell's equations for a monochromatic field can be written in the following form:

$$
\begin{aligned}
\frac{\mathrm{d}}{\mathrm{d} z} \boldsymbol{E}(z) & =-\frac{\mathrm{i} \omega}{c} \mu(z)[\boldsymbol{n} \times \boldsymbol{H}(z)], \\
\frac{\mathrm{d}}{\mathrm{d} z} \boldsymbol{H}(z) & =-\frac{4 \pi}{c} \sigma(z)[\boldsymbol{n} \times \boldsymbol{E}(z)],
\end{aligned}
$$

with boundary conditions

$$
\boldsymbol{E}\left(z=-d / 2-l_{0}\right)=\boldsymbol{E}_{1}, \quad \boldsymbol{H}\left(z=-d / 2-l_{0}\right)=\boldsymbol{H}_{1},
$$

where $\boldsymbol{E}_{1}$ and $\boldsymbol{H}_{1}$ are parallel to the film plane. Here the conductivity $\sigma(z)$ is equal to the metal conductivity $\sigma_{\mathrm{m}}$ inside the film $(-d / 2<z<d / 2)$, and to $\sigma_{\mathrm{d}}=-\mathrm{i} \omega / 4 \pi$ outside the film $(z<-d / 2$ and $z>d / 2)$, and similarly, the magnetic permeability $\mu(z)$ is equal to the film permeability $\mu_{\mathrm{m}}$ inside the film and to 1 outside the film; the unit vector $\boldsymbol{n}=\{0,0,1\}$ is perpendicular to the film plane. When solving Eqs. (7.72) and (7.73) it is taken into account that the electric and magnetic fields are continuous at the film boundaries. In this way the fields $\boldsymbol{E}(z)$ and $\boldsymbol{H}(z)$ are determined everywhere. Then electric $\boldsymbol{j}_{E}$ and magnetic $\boldsymbol{j}_{H}$ current flowing in-between the two planes at $z=-d / 2-l_{0}$ and at $z=d / 2+l_{0}$ are calculated as

$$
\begin{gathered}
\boldsymbol{j}_{E}=-\frac{\mathrm{i} \omega}{4 \pi}\left[\int_{-d / 2-l_{0}}^{-d / 2} \boldsymbol{E}(z) \mathrm{d} z+\int_{-d / 2}^{d / 2} \varepsilon_{\mathrm{m}} \boldsymbol{E}(z) \mathrm{d} z+\int_{d / 2}^{d / 2+l_{0}} \boldsymbol{E}(z) \mathrm{d} z\right], \\
\boldsymbol{j}_{H}=\frac{\mathrm{i} \omega}{4 \pi}\left[\int_{-d / 2-l_{0}}^{-d / 2} \boldsymbol{H}(z) \mathrm{d} z+\int_{-d / 2}^{d / 2} \mu_{\mathrm{m}} \boldsymbol{H}(z) \mathrm{d} z+\int_{d / 2}^{d / 2+l_{0}} \boldsymbol{H}(z) \mathrm{d} z\right],
\end{gathered}
$$


where $\varepsilon_{\mathrm{m}}=4 \mathrm{i} \pi \sigma_{\mathrm{m}} / \omega$ is the metal dielectric constant. Below it is supposed, for simplicity, that the magnetic permeability $\mu_{\mathrm{m}}=1$. Since the Maxwell equations are linear the local fields $\boldsymbol{E}(z)$ and $\boldsymbol{H}(z)$ are linear functions of the boundary values $\boldsymbol{E}_{1}$ and $\boldsymbol{H}_{1}$ defined at the plane $z=-d / 2-l_{0}$ :

$$
\begin{aligned}
& \boldsymbol{E}(z)=a(z) \boldsymbol{E}_{1}+c(z)\left[\boldsymbol{n} \times \boldsymbol{H}_{1}\right], \\
& \boldsymbol{H}(z)=b(z) \boldsymbol{H}_{1}+d(z)\left[\boldsymbol{n} \times \boldsymbol{E}_{1}\right] .
\end{aligned}
$$

Note that $\boldsymbol{n}$ is the single constant vector in the problem, which allows us to build polar $\left[\boldsymbol{n} \times \boldsymbol{H}_{1}\right]$ and axial $\left[\boldsymbol{n} \times \boldsymbol{E}_{1}\right]$ vectors in Eqs. (7.77) and (7.78). By substituting Eqs. (7.77) for $\boldsymbol{E}(z)$ and (7.78) for $\boldsymbol{H}(z)$ in Eqs. (7.75) and (7.76) correspondingly we express the currents $\boldsymbol{j}_{E}$ and $\boldsymbol{j}_{H}$ in terms of the boundary (surface) fields $\boldsymbol{E}_{1}$ and $\boldsymbol{H}_{1}$ as

$$
\begin{aligned}
& \boldsymbol{j}_{E}=s \boldsymbol{E}_{1}+g_{1}\left[\boldsymbol{n} \times \boldsymbol{H}_{1}\right], \\
& \boldsymbol{j}_{\boldsymbol{H}}=m \boldsymbol{H}_{1}+g_{2}\left[\boldsymbol{n} \times \boldsymbol{E}_{1}\right] .
\end{aligned}
$$

In contrast to the usual constitutive equations, the planar electric current $\boldsymbol{j}_{E}$, which flows between the planes $z=-d / 2-l_{0}$ and $z=d / 2+l_{0}$, depends not only on the external electric field $\boldsymbol{E}_{1}$ but also on the external magnetic field $\boldsymbol{H}_{1}$. The same is true for the magnetic induction current $\boldsymbol{j}_{\boldsymbol{H}}$. These equations constituted the GOL. The Ohmic parameters s, $\mathrm{m}, g_{1}$ and $g_{2}$ have the dimension of surface conductivity $(\mathrm{cm} / \mathrm{s})$ and depend on the frequency $\omega$, the metal dielectric constant $\varepsilon_{\mathrm{m}}$, the film thickness $d$ and the distance $l_{0}$ between the film and the reference plane $z=-d / 2-l_{0}$. Below the films are supposed to be invariant under reflection through the plane $z=0$. In this case $g_{1}=g_{2}=g$ as it is shown in [96], and the Ohmic the parameter $g$ can be expressed in terms of parameters $s$ and $m$ as

$$
g=-\frac{c}{4 \pi}+\sqrt{\left(\frac{c}{4 \pi}\right)^{2}-m s} .
$$

Then the GOL equations (7.79) and (7.80) take the following form:

$$
\begin{aligned}
\boldsymbol{j}_{E} & =s \boldsymbol{E}_{1}+g\left[\boldsymbol{n} \times \boldsymbol{H}_{1}\right], \\
\boldsymbol{j}_{H} & =m \boldsymbol{H}_{1}+g\left[\boldsymbol{n} \times \boldsymbol{E}_{1}\right],
\end{aligned}
$$

where the Ohmic parameter $g$ is given by Eq. (7.81). The Ohmic parameters $s$ and $m$ can be expressed in terms of the film refractive index $n=\sqrt{\varepsilon_{\mathrm{m}}}$ and film thickness $d$ in the following way:

$$
\begin{aligned}
& s=\frac{c}{8 n \pi}\left[\mathrm{e}^{-\mathrm{i} d k n}(n \cos (a d k)-\mathrm{i} \sin (a d k))^{2}-\mathrm{e}^{\mathrm{i} d k n}(n \cos (a d k)+\mathrm{i} \sin (a d k))^{2}\right], \\
& m=\frac{c}{8 n \pi}\left[\mathrm{e}^{-\mathrm{i} d k n}(\mathrm{i} \cos (a d k)+n \sin (a d k))^{2}-\mathrm{e}^{\mathrm{i} d k n}(-\mathrm{i} \cos (a d k)+n \sin (a d k))^{2}\right],
\end{aligned}
$$

where $k=\omega / c$; we still assume, for simplicity, that $\varepsilon=1$ outside the film $(z<-d / 2, z>d / 2)$ and introduce dimensionless parameter $a \equiv l_{0} / d$ (see [96,97]). The skin (penetration) depth $\delta$ is equal to $\delta=1 / k \operatorname{Im} n$ in these notations. In the microwave spectral range metal conductivity is real and 
dielectric constant $\varepsilon_{\mathrm{m}}$ is purely imaginary so that the skin depth $\delta=c / \sqrt{2 \pi \sigma_{\mathrm{m}} \omega}$; on the other hand the dielectric constant is negative for a typical metal in the optical and infrared spectra ranges, therefore $\delta \cong 1 / k \sqrt{\left|\varepsilon_{\mathrm{m}}\right|}$ in this case.

We now turn to the case of laterally inhomogeneous films. Then the currents $\boldsymbol{j}_{E}$ and $\boldsymbol{j}_{H}$ defined by Eqs. (7.75) and (7.76), as well as the fields $\boldsymbol{E}_{1}$ and $\boldsymbol{H}_{1}$ are functions of the two dimensional vector $\boldsymbol{r}=\{x, y\}$. From Maxwell's equations it follows that the fields and currents are connected by some linear relations:

$$
\begin{aligned}
& \boldsymbol{j}_{E}(\boldsymbol{r})=s \boldsymbol{E}_{1}+g\left[\boldsymbol{n} \times \boldsymbol{H}_{1}\right], \\
& \boldsymbol{j}_{H}(\boldsymbol{r})=m \boldsymbol{H}_{1}+g\left[\boldsymbol{n} \times \boldsymbol{E}_{1}\right],
\end{aligned}
$$

where $s, m$ and $g$ are some integral operators now. The metal islands in semicontinuous films usually have an oblate shape so that the grain diameter $D$ is much larger than the film thickness $d$ (see, e.g., [84]). When the thickness of a conducting grain $d$ (or skin depth $\delta$ ) and distance $l_{0}$ are much smaller than the grain diameter $D$, the relation of the fields $\boldsymbol{E}_{1}$ and $\boldsymbol{H}_{1}$ to the currents becomes fully local in Eqs. (7.86) and (7.87). The local Ohmic parameters $s=s(\boldsymbol{r}), m=m(\boldsymbol{r})$, and $g=g(\boldsymbol{r})$ given by Eqs. (7.81), (7.84) and (7.85) are determined by the local refractive index $n(\boldsymbol{r})=\sqrt{\varepsilon(\boldsymbol{r})}$, where $\varepsilon(\boldsymbol{r})$ is a local dielectric constant. Eqs. (7.86) and (7.87) are the local GOL for semicontinuous films. For binary metal-dielectric semicontinuous films the local dielectric constant is equal to either $\varepsilon_{\mathrm{m}}$ or $\varepsilon_{\mathrm{d}}$. The electric $\boldsymbol{j}_{E}$ and magnetic $\boldsymbol{j}_{H}$ currents given by Eqs. (7.86) and (7.87) lie in between the planes $z=-d / 2-l_{0}$ and $z=d / 2+l_{0}$. These currents satisfy the two-dimensional continuity equations

$$
\nabla \cdot \boldsymbol{j}_{E}(\boldsymbol{r})=0, \quad \nabla \cdot \boldsymbol{j}_{H}(\boldsymbol{r})=0,
$$

which follow from the three-dimensional continuity equations when the $z$-components of $\boldsymbol{E}_{1}$ and $\boldsymbol{H}_{1}$ are neglected at the planes $z= \pm\left(d / 2+l_{0}\right)$. This is possible because these components are small, in accordance with the fact that the average fields $\left\langle\boldsymbol{E}_{1}\right\rangle$ and $\left\langle\boldsymbol{H}_{1}\right\rangle$ are parallel to the film plane. Since we consider semicontinuous films with an inhomogeneity scale much smaller than the wavelength $\lambda$, the fields $\boldsymbol{E}_{1}(\boldsymbol{r})$ and $\boldsymbol{H}_{1}(\boldsymbol{r})$ are still the gradients of potential fields when considered as functions of $x$ and $y$ in the fixed reference plane $z=-d / 2-l_{0}$, i.e.,

$$
\boldsymbol{E}_{1}(\boldsymbol{r})=-\nabla \varphi_{1}(\boldsymbol{r}), \quad \boldsymbol{H}_{1}(\boldsymbol{r})=-\nabla \psi_{1}(\boldsymbol{r}) .
$$

By substituting these expressions in the continuity Eq. (7.88) and taking into account the GOL (7.86) and (7.87), the system of two basic equations for the electric $\varphi_{1}$ and magnetic $\psi_{1}$ potentials are obtained

$$
\nabla \cdot\left(s \nabla \varphi_{1}+g\left[\boldsymbol{n} \times \nabla \psi_{1}\right]\right)=0, \quad \nabla \cdot\left(m \nabla \psi_{1}+g\left[\boldsymbol{n} \times \nabla \varphi_{1}\right]\right)=0,
$$

where all variables are functions of the coordinates $x$ and $y$ in the reference plain. The above equations must be solved under the following conditions:

$$
\left\langle\nabla \varphi_{1}\right\rangle=\left\langle\boldsymbol{E}_{1}\right\rangle,\left\langle\nabla \psi_{1}\right\rangle=\left\langle\boldsymbol{H}_{1}\right\rangle,
$$

where the constant fields $\left\langle\boldsymbol{E}_{1}\right\rangle$ and $\left\langle\boldsymbol{H}_{1}\right\rangle$ are external (given) fields. Here and below $\langle\cdots\rangle$ denotes an average power coordinates " $x$ " and " $y$ ". 
The essence of the generalized Ohm's law can be summarized as follows. The entire physics of a three-dimensional inhomogeneous layer, which is described by the full set of Maxwell's equations, has been reduced to a set of quasistatic equations in a (two-dimensional) reference plane. Part of the price for this achievement is the introduction of coupled electric/magnetic fields and currents and dependence on one adjustable parameter, namely, the distance $l_{0}$ to the reference plane. Comparison of numerical calculation and GOL approximation for the metal film with periodic corrugation [97] show that GOL results are not sensitive to the distance $l_{0}$ in general. The original choice $l_{0}=0.25 D$ [96] [i.e., parameter $a=D / 4 d$ in Eqs. (7.84) and (7.85)] allows to reproduce most of the computer simulations except those where a surface polariton is excited in the corrugated film.

\subsubsection{Diagonalization of GOL equations}

To simplify the system of the basic equations (7.90) the electric and magnetic fields on both sides of the film are considered [39,97]. Namely, the electric and magnetic fields are considered at a distance $l_{0}$ behind the film $\boldsymbol{E}_{1}(\boldsymbol{r})=\boldsymbol{E}\left(\boldsymbol{r},-d / 2-l_{0}\right), \boldsymbol{H}_{1}(\boldsymbol{r})=\boldsymbol{H}\left(\boldsymbol{r},-d / 2-l_{0}\right)$, and at a distance $l_{0}$ in front of the film $\boldsymbol{E}_{2}(\boldsymbol{r})=\boldsymbol{E}\left(\boldsymbol{r}, d / 2+l_{0}\right), \boldsymbol{H}_{2}(\boldsymbol{r})=\boldsymbol{H}\left(\boldsymbol{r}, d / 2+l_{0}\right)$. Recall that $\boldsymbol{r}=\{x, y\}$ is two-dimensional vector in a plane perpendicular to " $z$ " axis. The components of the fields aligned with " $z$ " are still neglected. Then second Maxwell's equation curl $\boldsymbol{H}=(4 \pi / c) \boldsymbol{j}$ can be written as $\oint \boldsymbol{H} \mathrm{d} \boldsymbol{l}=(4 \pi / c)\left(\boldsymbol{n}_{1} \cdot \boldsymbol{j}_{E}\right) \Delta$, where the integration is over the rectangular contour, which has sides $d+2 l_{0}$ and $\Delta$ so that the sides $d+2 l_{0}$ are perpendicular to the film and sides $\Delta$ are in the planes $z= \pm\left(d / 2+l_{0}\right)$; vector $\boldsymbol{n}_{1}$ is perpendicular to the contour. When $\Delta \rightarrow 0$ this equation takes the following form:

$$
\boldsymbol{H}_{2}-\boldsymbol{H}_{1}=-\frac{4 \pi}{c}\left[\boldsymbol{n} \times \boldsymbol{j}_{E}\right]=-\frac{4 \pi}{c}\left(s\left[\boldsymbol{n} \times \boldsymbol{E}_{1}\right]-g \boldsymbol{H}_{1}\right),
$$

where the current $\boldsymbol{j}_{E}$ is given by the GOL Eq. (7.86). The same procedure being applied to the first Maxwell equation $\operatorname{curl} \boldsymbol{H}=\mathrm{i} k \boldsymbol{H}$ gives

$$
\boldsymbol{E}_{2}-\boldsymbol{E}_{1}=-\frac{4 \pi}{c}\left[\boldsymbol{n} \times \boldsymbol{j}_{H}\right]=-\frac{4 \pi}{c}\left(m\left[\boldsymbol{n} \times \boldsymbol{H}_{1}\right]-g \boldsymbol{E}_{1}\right),
$$

where the GOL equation (7.87) has been substituted for the electric current $\boldsymbol{j}_{H}$. Then electric field $\boldsymbol{E}_{1}$ can be expressed from Eq. (7.92) in terms of the magnetic fields $\boldsymbol{H}_{1}$ and $\boldsymbol{H}_{2}$ as

$$
\left[\boldsymbol{n} \times \boldsymbol{E}_{1}\right]=\frac{g}{s} \boldsymbol{H}_{1}-\frac{c}{4 \pi s}\left(\boldsymbol{H}_{2}-\boldsymbol{H}_{1}\right),
$$

magnetic field $\boldsymbol{H}_{1}$ can be expressed from Eq. (7.93) in terms of the electric fields $\boldsymbol{E}_{1}$ and $\boldsymbol{E}_{2}$ as

$$
\left[\boldsymbol{n} \times \boldsymbol{H}_{1}\right]=\frac{g}{m} \boldsymbol{E}_{1}-\frac{c}{4 \pi m}\left(\boldsymbol{E}_{2}-\boldsymbol{E}_{1}\right) .
$$

Substituting the r.h.s. of Eq. (7.94) in the GOL Eq. (7.87) and substituting Eq. (7.95) in the GOL Eq. (7.86) results in

$$
\boldsymbol{j}_{E}=s \boldsymbol{E}_{1}+g\left(\frac{g}{m} \boldsymbol{E}_{1}-\frac{c}{4 \pi m}\left(\boldsymbol{E}_{2}-\boldsymbol{E}_{1}\right)\right),
$$




$$
\boldsymbol{j}_{H}=m \boldsymbol{H}_{1}+g\left(\frac{g}{s} \boldsymbol{H}_{1}-\frac{c}{4 \pi s}\left(\boldsymbol{H}_{2}-\boldsymbol{H}_{1}\right)\right) .
$$

Finally the relation (7.81) between the Ohmic parameters $s, m$ and $g$ allows us to rewrite the above equations as

$$
\boldsymbol{j}_{E}=u \boldsymbol{E}, \quad \boldsymbol{j}_{H}=w \boldsymbol{H},
$$

where $\boldsymbol{E}=\left(\boldsymbol{E}_{1}+\boldsymbol{E}_{2}\right) / 2, \boldsymbol{H}=\left(\boldsymbol{H}_{1}+\boldsymbol{H}_{2}\right) / 2$ and parameters $u$ and $w$ are given by the following equations:

$$
u=-\frac{c}{2 \pi} \frac{g}{m}, \quad w=-\frac{c}{2 \pi} \frac{g}{s} .
$$

Thus the GOL is diagonalized by introducing new fields $\boldsymbol{E}$ and $\boldsymbol{H}$ so that Eqs. (7.98) have the same form as constitutive equations of the macroscopic electrodynamics. The only difference is that the local conductivity $\sigma$ is replaced by parameter $u$ and magnetic permeability $\mu$ is replaced by parameter $-\mathrm{i} 4 \pi \mathrm{w} / \omega$.

It follows from Eqs. (7.99) and (7.81), (7.84), (7.85) that the new Ohmic parameters $u$ and $v$ are expressed in terms of the local refractive index $n=\sqrt{\varepsilon(\boldsymbol{r})}$ as

$$
\begin{aligned}
& u=-\mathrm{i} \frac{c}{2 \pi} \frac{\tan (D k / 4)+n \tan (d k n / 2)}{1-n \tan (D k / 4) \tan (d k n / 2)} \\
& w=\mathrm{i} \frac{c}{2 \pi} \frac{n \tan (D k / 4)+\tan (d k n / 2)}{n-\tan (D k / 4) \tan (d k n / 2)}
\end{aligned}
$$

where the parameter $a=D / 4 d$ is substituted as it is discussed at the end of the previous section. The refractive index $n$ in the above equations takes values $n_{\mathrm{m}}=\sqrt{\varepsilon_{\mathrm{m}}}$ and $n_{\mathrm{d}}=\sqrt{\varepsilon_{\mathrm{d}}}$ for metal and dielectric regions of the film, respectively. In the quasistatic limit, when the optical thickness of metal grains is small $\mathrm{d} k\left|n_{\mathrm{m}}\right| \ll 1$, while the metal dielectric constant is large in magnitude, $\left|\varepsilon_{\mathrm{m}}\right| \gg 1$, the following estimates hold:

$$
u_{\mathrm{m}} \simeq-\mathrm{i} \frac{\omega \varepsilon_{\mathrm{m}}}{4 \pi} d, \quad w_{\mathrm{m}} \simeq \mathrm{i} \frac{\omega}{4 \pi}(d+D / 2) \quad(d / \delta \ll 1)
$$

for the metal grains. In the opposite case of strong skin effect, when the skin depth (penetration depth) $\delta=1 / k \operatorname{Im} n_{\mathrm{m}}$ is much smaller than the grain thickness $d$ and the electromagnetic field does not penetrate in metal grains, the parameters $u_{\mathrm{m}}$ and $w_{\mathrm{m}}$ take values

$$
u_{\mathrm{m}}=\mathrm{i} \frac{2 c^{2}}{\pi D \omega}, \quad w_{\mathrm{m}}=\mathrm{i} \frac{\omega D}{8 \pi} \quad(d / \delta \gg 1) .
$$

For the dielectric region, when the film is thin enough so that $\mathrm{d} k n_{\mathrm{d}} \ll 1$, and $\varepsilon_{\mathrm{d}} \sim 1$ Eqs. (7.100) and (7.101) give

$$
u_{\mathrm{d}}=-\mathrm{i} \frac{\omega \varepsilon_{\mathrm{d}}^{\prime}}{8 \pi} D, \quad w_{\mathrm{d}}=\mathrm{i} \frac{\omega}{4 \pi}(d+D / 2),
$$


where the reduced dielectric constant $\varepsilon_{\mathrm{d}}^{\prime}=1+2 \varepsilon_{\mathrm{d}} d / D$ is introduced. Note that in the limit of strong skin effect the Ohmic parameters $u_{\mathrm{m}}$ and $w_{\mathrm{m}}$ are purely imaginary and the parameter $u_{\mathrm{m}}$ is of inductive character, i.e., it has the sign opposite to the dielectric parameter $u_{\mathrm{d}}$. In contrast, the Ohmic parameter $w$ remains essentially the same $w \sim \mathrm{i} D \omega / 8 \pi$ for dielectric and for metal regions regardless of the value of the skin effect.

Potentials for the fields $\boldsymbol{E}_{2}(\boldsymbol{r})$ and $\boldsymbol{H}_{2}(\boldsymbol{r})$ can be introduced for the same reason as potentials for the fields $\boldsymbol{E}_{1}(\boldsymbol{r})$ and $\boldsymbol{H}_{1}(\boldsymbol{r})$ [see discussion accompanying Eq. (7.89)]. Therefore, the fields $\boldsymbol{E}(\boldsymbol{r})$ and $\boldsymbol{H}(\boldsymbol{r})$ in Eqs. (7.98) can in turn be represented as gradients of some potentials:

$$
\boldsymbol{E}=-\nabla \phi^{\prime}, \quad \boldsymbol{H}=-\nabla \psi^{\prime} .
$$

By substituting these expressions in Eqs. (7.98) and then in the continuity Eqs. (7.88), we obtain the following equations:

$$
\begin{aligned}
& \nabla \cdot\left[u(\boldsymbol{r}) \nabla \varphi^{\prime}(\boldsymbol{r})\right]=0, \\
& \nabla \cdot\left[w(\boldsymbol{r}) \nabla \psi^{\prime}(\boldsymbol{r})\right]=0,
\end{aligned}
$$

which can be solved independently for the potentials $\varphi^{\prime}$ and $\psi^{\prime}$. The above equations are solved under the following conditions:

$$
\left\langle\nabla \varphi_{1}^{\prime}\right\rangle=\langle\boldsymbol{E}\rangle \equiv \boldsymbol{E}_{0},\left\langle\nabla \psi_{1}^{\prime}\right\rangle=\left\langle\boldsymbol{H}_{1}\right\rangle \equiv \boldsymbol{H}_{0},
$$

where the constant fields $\boldsymbol{E}_{0}$ and $\boldsymbol{H}_{0}$ are external (given) fields that are determined by the incident wave. When the fields $\boldsymbol{E}, \boldsymbol{H}$ and currents $\boldsymbol{j}_{\boldsymbol{E}}, \boldsymbol{j}_{H}$ are found from a solution of Eqs. (7.106), (7.107) and (7.108), the local electric and magnetic fields in the plane $z=-l_{0}-d / 2$ are given by the equations

$$
\boldsymbol{E}_{1}=\boldsymbol{E}+\frac{2 \pi}{c}\left[\boldsymbol{n} \times \boldsymbol{j}_{H}\right], \quad \boldsymbol{H}_{1}=\boldsymbol{H}+\frac{2 \pi}{c}\left[\boldsymbol{n} \times \boldsymbol{j}_{E}\right]
$$

that follow from Eqs. (7.92) and (7.93) and definitions of the fields $\boldsymbol{E}$ and $\boldsymbol{H}$. Note that the field $\boldsymbol{E}_{1}(\boldsymbol{r})$ can be measured in a near field experiment. (For a comprehensive review see, e.g., [137].) The effective parameters $u_{\mathrm{e}}$ and $w_{\mathrm{e}}$ are defined in a usual way

$$
\begin{aligned}
& \left\langle\boldsymbol{j}_{E}\right\rangle=u_{\mathrm{e}} \boldsymbol{E}_{0} \equiv u_{\mathrm{e}}\left(\left\langle\boldsymbol{E}_{1}\right\rangle+\left\langle\boldsymbol{E}_{2}\right\rangle\right) / 2, \\
& \left\langle\boldsymbol{j}_{H}\right\rangle=w_{\mathrm{e}} \boldsymbol{H}_{0} \equiv w_{\mathrm{e}}\left(\left\langle\boldsymbol{H}_{1}\right\rangle+\left\langle\boldsymbol{H}_{2}\right\rangle\right) / 2 .
\end{aligned}
$$

These expressions are substituted in Eqs. (7.92) and (7.93), which are averaged over the $\{x, y\}$ coordinates to obtain equations

$$
\begin{aligned}
& {\left[\boldsymbol{n} \times\left(\left\langle\boldsymbol{H}_{2}\right\rangle-\left\langle\boldsymbol{H}_{1}\right\rangle\right)\right]=\frac{2 \pi}{c} u_{\mathrm{e}}\left(\left\langle\boldsymbol{E}_{1}\right\rangle+\left\langle\boldsymbol{E}_{2}\right\rangle\right),} \\
& {\left[\boldsymbol{n} \times\left(\left\langle\boldsymbol{E}_{2}\right\rangle-\left\langle\boldsymbol{E}_{1}\right\rangle\right)\right]=\frac{2 \pi}{c} w_{\mathrm{e}}\left(\left\langle\boldsymbol{H}_{1}\right\rangle+\left\langle\boldsymbol{H}_{2}\right\rangle\right),}
\end{aligned}
$$

for the averaged fields that determine the optical response of an inhomogeneous film.

Let us suppose that the wave enters the film from the right half-space (see Fig. 24), so that its amplitude is proportional to $\mathrm{e}^{-\mathrm{i} k z}$. The incident wave is partially reflected and partially transmitted 
through the film. The electric field amplitude in the right half-space, away from the film, can be written as $\boldsymbol{e}\left[\mathrm{e}^{-\mathrm{i} k z}+r \mathrm{e}^{\mathrm{i} k z}\right]$, where $r$ is the reflection coefficient and $\boldsymbol{e}$ is the polarization vector. Then the electric component of the electromagnetic wave well behind the film acquires the form $e t \mathrm{e}^{-\mathrm{i} k z}$, where $t$ is the transmission coefficient. It is supposed for simplicity that the film has no optical activity. Therefore wave polarization $\boldsymbol{e}$ remains the same before and after the film. At the planes $z=d / 2+l_{0}$ and $z=-d / 2-l_{0}$ the average electric field equals $\left\langle\boldsymbol{E}_{2}\right\rangle$ and $\left\langle\boldsymbol{E}_{1}\right\rangle$, respectively. Now the wave away from the film is matched with the average fields in the planes $z=d / 2+l_{0}$ and $z=-d / 2-l_{0}$, i.e., $\left\langle\boldsymbol{E}_{2}\right\rangle=\boldsymbol{e}\left[\mathrm{e}^{-\mathrm{i} k\left(d / 2+l_{0}\right)}+r \mathrm{e}^{\mathrm{i} k\left(d / 2+l_{0}\right)}\right]$ and $\left\langle\boldsymbol{E}_{1}\right\rangle=\boldsymbol{e} t \mathrm{e}^{\mathrm{i} k\left(d / 2+l_{0}\right)}$. The same matching but with magnetic fields gives $\left\langle\boldsymbol{H}_{2}\right\rangle=[\boldsymbol{n} \times \boldsymbol{e}]\left[-\mathrm{e}^{-\mathrm{i} k\left(d / 2+l_{0}\right)}+r \mathrm{e}^{\mathrm{i} k\left(d / 2+l_{0}\right)}\right]$ and $\left\langle\boldsymbol{H}_{1}\right\rangle=-[\boldsymbol{n} \times \boldsymbol{e}] t \mathrm{e}^{\mathrm{i} k\left(d / 2+l_{0}\right)}$ in the planes $z=d / 2+l_{0}$ and $z=-d / 2-l_{0}$, respectively. Substitution of these expressions for the fields $\left\langle\boldsymbol{E}_{1}\right\rangle,\left\langle\boldsymbol{E}_{2}\right\rangle,\left\langle\boldsymbol{H}_{1}\right\rangle$ and $\left\langle\boldsymbol{H}_{2}\right\rangle$ in Eqs. (7.112) and (7.113) gives two scalar, linear equations for reflection $r$ and transmission $t$ coefficients. Solution to these equations gives the reflectance,

$$
R \equiv|r|^{2}=\left|\frac{\frac{2 \pi}{c}\left(u_{\mathrm{e}}+w_{\mathrm{e}}\right)}{\left(1+\frac{2 \pi}{c} u_{\mathrm{e}}\right)\left(1-\frac{2 \pi}{c} w_{\mathrm{e}}\right)}\right|^{2},
$$

transmittance

$$
T \equiv|t|^{2}=\left|\frac{1+\left(\frac{2 \pi}{c}\right)^{2} u_{\mathrm{e}} w_{\mathrm{e}}}{\left(1+\frac{2 \pi}{c} u_{\mathrm{e}}\right)\left(1-\frac{2 \pi}{c} w_{\mathrm{e}}\right)}\right|^{2}
$$

and absorbance

$$
A=1-T-R
$$

of the film. Therefore, the effective Ohmic parameters $u_{\mathrm{e}}$ and $w_{\mathrm{e}}$ determine completely the optical properties of an inhomogeneous films.

Thus the problem of the field distribution and optical properties of the metal-dielectric films reduces to uncoupled quasistatic conductivity problems [Eq. (7.107)] to which extensive theory already exists. Thus numerous analytical as well as numerical methods developed in the percolation theory can be used to find the effective parameters $u_{\mathrm{e}}$ and $w_{\mathrm{e}}$ of the film (see Section 3).

Let us consider now the case of the strong skin effect in metal grains and trace the evolution of the optical properties of a semicontinuous metal film when the surface density $p$ of the metal is increasing. When $p=0$ the film is purely dielectric and the effective parameters $u_{\mathrm{e}}$ and $w_{\mathrm{e}}$ coincide with the dielectric Ohmic parameters given by Eqs. (7.104). By substituting $u_{\mathrm{e}}=u_{\mathrm{d}}$ and $w_{\mathrm{e}}=w_{\mathrm{d}}$ in Eqs. (7.114), (7.115) and (7.116) and assuming that the dielectric film has no losses and it is optically thin $\left(d k \varepsilon_{\mathrm{d}} \ll 1\right)$, we obtain the reflectance $R=d^{2}\left(\varepsilon_{\mathrm{d}}-1\right)^{2} k^{2} / 4$, transmittance $T=1-d^{2}\left(\varepsilon_{\mathrm{d}}-1\right)^{2} k^{2} / 4$, and the absorbance $A=0$ that coincide with the well known results for a thin dielectric film $[82,130]$.

It is not surprising that the film without losses has zero absorbance. When the ratio of the penetration length (skin depth) $\delta=1 / k \operatorname{Im} n_{\mathrm{m}}$ is negligible in comparison with the film thickness 
$d$ and $\left|n_{\mathrm{m}}\right| \gg 1$ the losses are also absent in the limit of full coverage, when the metal concentration $p=1$. In this case the film is a perfect metal mirror. Indeed substituting the Ohmic parameters $u_{\mathrm{e}}=u_{\mathrm{m}}$ and $w_{\mathrm{e}}=w_{\mathrm{m}}$ from Eqs. (7.103) in Eqs. (7.114), (7.115) and (7.116) we obtain for the reflectance $R=1$, while the transmittance $T$ and absorbance $A$ are both equal to zero. Note that the optical properties of the film do not depend on the particle size $D$ for the metal concentration $p=0$ and $p=1$ since properties of the dielectric and continuous metal films do not depend on the shape of the metal grains.

We consider now the film at the percolation threshold $p=p_{c}$ with $p_{c}=1 / 2$ for a self-dual system $[12,20]$. A semicontinuous metal film may be thought of as a mirror, which is broken into small pieces with typical size $D$ much smaller than the wavelength $\lambda$. At the percolation threshold the exact Dykhne formulas $u_{\mathrm{e}}=\sqrt{u_{\mathrm{d}} u_{\mathrm{m}}}, w_{\mathrm{e}}=\sqrt{w_{\mathrm{d}} w_{\mathrm{m}}}$ hold [100]. Thus the following equations for the effective Ohmic parameters are obtained from Eqs. (7.104) and (7.103)

$$
\frac{2 \pi}{c} u_{\mathrm{e}}\left(p_{c}\right)=\sqrt{\varepsilon_{\mathrm{d}}^{\prime}}, \quad \frac{2 \pi}{c} w_{\mathrm{e}}\left(p_{c}\right)=\mathrm{i} \frac{D k}{4} \sqrt{1+\frac{2 d}{D}} .
$$

From this equation it follows that $\left|w_{\mathrm{e}} / u_{\mathrm{e}}\right| \sim D k \ll 1$ and the effective Ohmic parameter $w_{\mathrm{e}}$ can be neglected in comparison with $u_{\mathrm{e}}$. By substituting the effective Ohmic parameter $u_{\mathrm{e}}\left(p_{c}\right)$ given by Eq. (7.117) in Eqs. (7.114), (7.115) and (7.116) the optical properties at the percolation are obtained

$$
\begin{aligned}
& R\left(p_{c}\right)=\varepsilon_{\mathrm{d}}^{\prime} /\left(1+\sqrt{\varepsilon_{\mathrm{d}}^{\prime}}\right)^{2}, \\
& T\left(p_{c}\right)=1 /\left(1+\sqrt{\varepsilon_{\mathrm{d}}^{\prime}}\right)^{2}, \\
& A\left(p_{c}\right)=2 \sqrt{\varepsilon_{\mathrm{d}}^{\prime}} /\left(1+\sqrt{\varepsilon_{\mathrm{d}}^{\prime}}\right)^{2} ;
\end{aligned}
$$

recall that the reduced dielectric function $\varepsilon_{\mathrm{d}}^{\prime}=1+2 \varepsilon_{\mathrm{d}} d / D$. When metal grains are oblate enough so that $\varepsilon_{\mathrm{d}} d / D \ll 1$ and $\varepsilon_{\mathrm{d}}^{\prime} \rightarrow 1$ the above expressions simplify to the universal result

$$
R=T=1 / 4, \quad A=1 / 2 \text {. }
$$

Thus, there is effective adsorption in semicontinuous metal films even for the case when neither dielectric nor metal grains absorb light energy. The mirror broken into small pieces effectively absorbs energy from the electromagnetic field. The effective absorption in a loss-free film means that the electromagnetic energy is stored in the system and that the amplitudes of the local electromagnetic field increase up to infinity. In any real semicontinuous metal film the local field saturates due to non-zero losses, but the significant field fluctuations take place over the film when losses are small, as discussed below.

To find the optical properties of semicontinuous films for arbitrary metal concentration $p$ the effective medium theory can be implemented and this had been originally developed to provide a semi-quantitative description of the transport properties of percolating composites [12]. The effective medium theory being applied to Eqs. (7.106), (7.110) and (7.107), (7.111) results in the following equations for the effective parameters:

$$
\begin{aligned}
& u_{\mathrm{e}}^{2}-\Delta p u_{\mathrm{e}}\left(u_{\mathrm{m}}-u_{\mathrm{d}}\right)-u_{\mathrm{d}} u_{\mathrm{m}}=0, \\
& w_{\mathrm{e}}^{2}-\Delta p w_{\mathrm{e}}\left(w_{\mathrm{m}}-w_{\mathrm{d}}\right)-w_{\mathrm{d}} w_{\mathrm{m}}=0,
\end{aligned}
$$


where the reduced concentration $\Delta p=\left(p-p_{c}\right) / p_{c}\left(p_{c}=1 / 2\right)$ is introduced. It follows from Eq. (7.123) that for the considered case of strong skin effect, when the Ohmic parameters $w_{\mathrm{m}}$ and $w_{\mathrm{d}}$ are given by Eqs. (7.103) and (7.104), the effective Ohmic parameter $\left|w_{\mathrm{e}}\right| \ll c$ for all metal concentrations $p$. Therefore, the parameter $w_{\mathrm{e}}$ is negligible in Eqs. (7.114) and (7.115). For further simplification the Ohmic parameter $u_{\mathrm{d}}$ can be neglected in comparison with $u_{\mathrm{m}}$ in the second term of Eq. (7.122) [cf. Eqs. (7.103) and (7.104)]. Then introduction of the dimensionless Ohmic parameter $u_{\mathrm{e}}^{\prime}=(2 \pi / c) u_{\mathrm{e}}$ allows to rewrite Eq. (7.122) as

$$
u_{\mathrm{e}}^{\prime 2}-2 \mathrm{i} \frac{\lambda \Delta p}{\pi D} u_{\mathrm{e}}^{\prime}-\varepsilon_{\mathrm{d}}^{\prime}=0 .
$$

Right at the percolation threshold $p=p_{c}=1 / 2$, when the reduced concentration $\Delta p=0$, Eq. (7.124) gives the effective Ohmic parameter $u_{\mathrm{e}}^{\prime}\left(p_{c}\right)=\sqrt{\varepsilon_{\mathrm{d}}^{\prime}}$ that coincides with the exact Eq. (7.117) and results in reflectance, transmittance and absorbance given by Eqs. (7.118), (7.119) and (7.120), respectively. For concentrations different from the percolation threshold, Eq. (7.124) gives

$$
u_{\mathrm{e}}^{\prime}=\mathrm{i} \frac{\lambda \Delta p}{\pi D}+\sqrt{-\left(\frac{\lambda \Delta p}{\pi D}\right)^{2}+\varepsilon_{\mathrm{d}}^{\prime}},
$$

which becomes purely imaginary for $|\Delta p|>\pi D \sqrt{\varepsilon_{\mathrm{d}}^{\prime}} / \lambda$. Then Eqs. (7.118), (7.119) and (7.120) result in the zero absorbance $A=1-R-T=1-\left|u_{\mathrm{e}}^{\prime}\right|^{2} /\left|1+u_{\mathrm{e}}^{\prime}\right|^{2}-1 /\left|1+u_{\mathrm{e}}^{\prime}\right|^{2}=0$ (recall that the effective Ohmic parameter $w_{\mathrm{e}}$ is neglected). In the vicinity of a percolation threshold, namely, for

$$
|\Delta p|<\pi D / \lambda \sqrt{\varepsilon_{\mathbf{d}}^{\prime}}
$$

the effective Ohmic parameter $u_{\mathrm{e}}^{\prime}$ has a nonvanishing real part and, therefore, the absorbance

$$
A=\frac{2 \sqrt{-\left(\frac{\lambda \Delta p}{\pi D}\right)^{2}+\varepsilon_{\mathrm{d}}^{\prime}}}{1+\varepsilon_{\mathrm{d}}^{\prime}+2 \sqrt{-\left(\frac{\lambda \Delta p}{\pi D}\right)^{2}+\varepsilon_{\mathrm{d}}^{\prime}}}
$$

is nonzero and has a well-defined maximum at the percolation threshold; the width of the maximum is inversely proportional to the wavelength. The effective absorption in almost loss-free semicontinuous metal film means that local electromagnetic fields strongly fluctuate in the system as was speculated above. The spectral width for the strong fluctuations should be the same as the width of the absorption maximum, i.e. it is given by Eq. (7.126).

Note that the effective parameters $u_{\mathrm{e}}$ and $w_{\mathrm{e}}$ can be determined experimentally by measuring the amplitude and phase of the transmitted and reflected waves using, for example, a waveguide technique (see, Ref. [136] and references therein), or by measuring the film reflectance as function of the fields $\boldsymbol{E}_{1}$ and $\boldsymbol{H}_{1}$. In this case, a metal screen placed behind the film can be used to control the values of these fields $[138,139]$.

\subsubsection{Numerical simulation of local electric and magnetic fields}

To find the local electric $\boldsymbol{E}(\boldsymbol{r})$ and magnetic $\boldsymbol{H}(\boldsymbol{r})$ fields Eqs. (7.106) and (7.107) should be solved. Consider the first equation (7.106), which can be conveniently rewritten in terms of the 
dimensionless "dielectric constant"

$$
\tilde{\varepsilon}=4 \pi \mathrm{i} u(\boldsymbol{r}) / \omega d
$$

as follows

$$
\nabla \cdot[\tilde{\varepsilon}(\boldsymbol{r}) \nabla \phi(\boldsymbol{r})]=\mathscr{E},
$$

where $\phi(\boldsymbol{r})$ is the fluctuating part of the potential $\phi^{\prime}(\boldsymbol{r})$ so that $\nabla \phi^{\prime}(\boldsymbol{r})=\nabla \phi(\boldsymbol{r})-\boldsymbol{E}_{0},\langle\phi(\boldsymbol{r})\rangle=0$, and $\mathscr{E}=\nabla \cdot\left[\tilde{\varepsilon}(\boldsymbol{r}) \boldsymbol{E}_{0}\right]$. Recall that the "external" field $\boldsymbol{E}_{0}$ is defined by Eq. (7.108). For the metal-dielectric films considered here local dielectric constant $\tilde{\varepsilon}(\boldsymbol{r})$ equals $\left(\tilde{\varepsilon}_{\mathrm{m}}=\right) 4 \pi \mathrm{i} u_{\mathrm{m}} / \omega d$ and $\left(\tilde{\varepsilon}_{\mathrm{d}}=\right) \varepsilon_{\mathrm{d}}^{\prime} D / 2 d$ for the metal and dielectric regions, respectively. The external field $\boldsymbol{E}_{0}$ in Eq. (7.129) can be chosen real, while the local potential $\phi(r)$ takes complex values since the dielectric constant $\tilde{\varepsilon}_{\mathrm{m}}$ is complex $\tilde{\varepsilon}_{\mathrm{m}}=\tilde{\varepsilon}_{\mathrm{m}}^{\prime}+\mathrm{i} \tilde{\varepsilon}_{\mathrm{m}}^{\prime \prime}$.

In the quasistatic limit when the skin depth $\delta$ is much larger than the film thickness $d$ the dielectric constant $\tilde{\varepsilon}_{\mathrm{m}}$ coincides with the metal dielectric constant $\varepsilon_{\mathrm{m}}$ as it follows from Eq. (7.102). Recall that the real part of the metal dielectric constant $\varepsilon_{\mathrm{m}}^{\prime}$ becomes negative, $\varepsilon_{\mathrm{m}}^{\prime}<0$, for the frequency $\omega<\tilde{\omega}_{\mathrm{p}}$, where $\tilde{\omega}_{\mathrm{p}}$ is given by Eq. (2.2). This allows to model the metal grains as inductances $L$ for frequencies $\tilde{\omega}_{\mathrm{p}}>\omega \gg \omega_{\tau}$ while the dielectric gaps can be represented by capacitances $C$ (see discussion at the beginning of Section 3). In the opposite case of the strong skin effect, the Ohmic parameter $u_{\mathrm{m}}$ is inductive according to Eq. (7.103) for all spectral ranges regardless of the metal properties. Then, the percolation metal-dielectric film represents a set of randomly distributed $L$ and $C$ elements for all spectral ranges when the skin effect is strong in the metal grains. Note that Ohmic parameter $w$ takes the same sign and rather close absolute values for metal and dielectric grains according to Eqs. (7.102), (7.103) and (7.104). A film can be thought as a collection of $C$ elements in " $w$ " space. Therefore, the resonance phenomena are absent in a solution of Eq. (7.107). The fluctuations of the potential $\psi^{\prime}$ can indeed be neglected in comparison with the $\varphi^{\prime}$ fluctuations. For this reason we concentrate attention on the properties of the "electric" field $\boldsymbol{E}(\boldsymbol{r})=-\nabla \phi^{\prime}(\boldsymbol{r})=-\nabla \phi(\boldsymbol{r})+\boldsymbol{E}_{0}$ when considering the fluctuation of the local fields. The field $\boldsymbol{E}(\boldsymbol{r})$ can be found from the solution of Eq. (7.129).

Since Eq. (7.129) has the same form as Eq. (2.4) it can be discretized on a square lattice in the same way. Then numerical methods discussed in Section 3 can be used to find the local field distribution. The real space renormalization method described in Section 3.1 was employed to solve Eq. (2.5) and calculate the potentials $\phi_{i}$ in the lattice. This gives the local field $\boldsymbol{E}(\boldsymbol{r})$ and electric current $\boldsymbol{j}_{E}(\boldsymbol{r})$ in terms of the average field $\boldsymbol{E}_{0}$. The effective Ohmic parameter $u_{\mathrm{e}}$ is determined by Eq. (7.110) that can be written as $\left\langle\boldsymbol{j}_{E}\right\rangle=u_{\mathrm{e}} \boldsymbol{E}_{0}$. The effective dielectric constant $\tilde{\varepsilon}_{\mathrm{e}}$ equals $4 \pi \mathrm{i} u_{\mathrm{e}} / \omega d$. In the same manner the field $\boldsymbol{H}(\boldsymbol{r})$, the magnetic current $\boldsymbol{j}_{H}(\boldsymbol{r})$ and the effective parameter $w_{\mathrm{e}}$ can be found from Eq. (7.107) and its lattice discretization. Note that the same lattice should be used to determine the fields $\boldsymbol{E}(\boldsymbol{r})$ and $\boldsymbol{H}(\boldsymbol{r})$. The directions of the external fields $\boldsymbol{E}_{0}$ and $\boldsymbol{H}_{0}$ may be chosen arbitrarly when the effective parameters $u_{\mathrm{e}}$ and $w_{\mathrm{e}}$ are calculated since the effective parameters do not depend on the direction of the field for a film, which is isotropic as a whole.

Though the effective parameters do not depend on the external field the local electric $\boldsymbol{E}_{1}(\boldsymbol{r})$ and magnetic $\boldsymbol{H}_{1}(\boldsymbol{r})$ fields do depend on the incident wave. The local fields $\boldsymbol{E}_{1}(\boldsymbol{r})$ and $\boldsymbol{H}_{1}(\boldsymbol{r})$ are defined in the reference plane $z=-d / 2-l_{0}$ (see Fig. 24). Note that the field $\boldsymbol{E}_{1}(\boldsymbol{r})$ can be measured in a typical near field experiment (see, e.g., Ref. [137]). For the calculations below the electric and magnetic fields of the incident electromagnetic wave have been chosen in the form $\left\langle\boldsymbol{E}_{1}\right\rangle=\{1,0,0\}$ 
and $\left\langle\boldsymbol{H}_{1}\right\rangle=\{0,-1,0\}$ in the plane $z=-l_{0}-d / 2$. This choice corresponds to the wave vector of the incident wave as $\boldsymbol{k}=(0,0,-k)$, i.e., there is only a transmitted wave behind the film (see Fig. 24). It follows from the average of Eqs. (7.109), which can be written as $\left\langle\boldsymbol{E}_{1}\right\rangle=\boldsymbol{E}_{0}+$ $(2 \pi / c) w_{\mathrm{e}}\left[\boldsymbol{n} \times \boldsymbol{H}_{0}\right]$ and $\left\langle\boldsymbol{H}_{1}\right\rangle=\boldsymbol{H}_{0}+(2 \pi / c) u_{\mathrm{e}}\left[\boldsymbol{n} \times \boldsymbol{E}_{0}\right]$, that the fields $\boldsymbol{E}_{0}$ and $\boldsymbol{H}_{0}$ are given by

$$
\boldsymbol{E}_{0}=\frac{\left\langle\boldsymbol{E}_{1}\right\rangle-\frac{2 \pi}{c} w_{\mathrm{e}}\left[\boldsymbol{n} \times\left\langle\boldsymbol{H}_{1}\right\rangle\right]}{1+\left(\frac{2 \pi}{c}\right)^{2} u_{\mathrm{e}} w_{\mathrm{e}}}, \quad \boldsymbol{H}_{0}=\frac{\left\langle\boldsymbol{H}_{1}\right\rangle-\frac{2 \pi}{c} u_{\mathrm{e}}\left[\boldsymbol{n} \times\left\langle\boldsymbol{E}_{1}\right\rangle\right]}{1+\left(\frac{2 \pi}{c}\right)^{2} u_{\mathrm{e}} w_{\mathrm{e}}} .
$$

These values of the fields $\boldsymbol{E}_{0}$ and $\boldsymbol{H}_{0}$ are used to calculate the local fields $\boldsymbol{E}(\boldsymbol{r})$ and $\boldsymbol{H}(\boldsymbol{r})$. The local electric $\boldsymbol{E}_{1}(\boldsymbol{r})$ and magnetic $\boldsymbol{H}_{1}(\boldsymbol{r})$ fields are restored then from the fields $\boldsymbol{E}(\boldsymbol{r})$ and $\boldsymbol{H}(\boldsymbol{r})$ by using Eq. (7.109).

The local electric and magnetic fields have been calculated in silver-on-glass semicontinuous film as functions of the surface concentration $p$ of silver grains. The typical glass dielectric constant is about $\varepsilon_{\mathrm{d}}=2.2$. The dielectric function for silver was chosen in the Drude form (2.1). The following parameters were also used in Eq. (2.1): the interband-transition contribution $\varepsilon_{\mathrm{b}}=5$, the plasma frequency $\omega_{\mathrm{p}}=9.1 \mathrm{eV}$, and the relaxation frequency $\omega_{\tau}=0.021 \mathrm{eV}$ [104]. The metal grains are supposed to be oblate. The ratio of the grain thickness $d$ (film thickness) to the grain diameter $D$ has been chosen $(D / d=) 3$, the same as that used in Ref. [96]. To consider skin effect of different strength (i.e. different interactions between the electric and magnetic fields), we vary the size $d$ of silver particles in a wide range, $d=1-100 \mathrm{~nm}$. The size of metal grains in semicontinuous metal films is usually of the order of few nanometers but it can be increased significantly by using a proper method of preparation [108]. For microwave experiments [39] the films were prepared by lithography method so that the size of metal particle could vary in a large range.

The space distribution of the electric and magnetic fields was calculated at two sets of parameters as illustrated in Figs. 25-28. In Figs. 25 and 26 we show the electric and magnetic field distributions for $\lambda=1 \mu \mathrm{m}$ and two different thicknesses $d$ of the film, $d=5 \mathrm{~nm}$ and $d=50 \mathrm{~nm}$. The first thickness
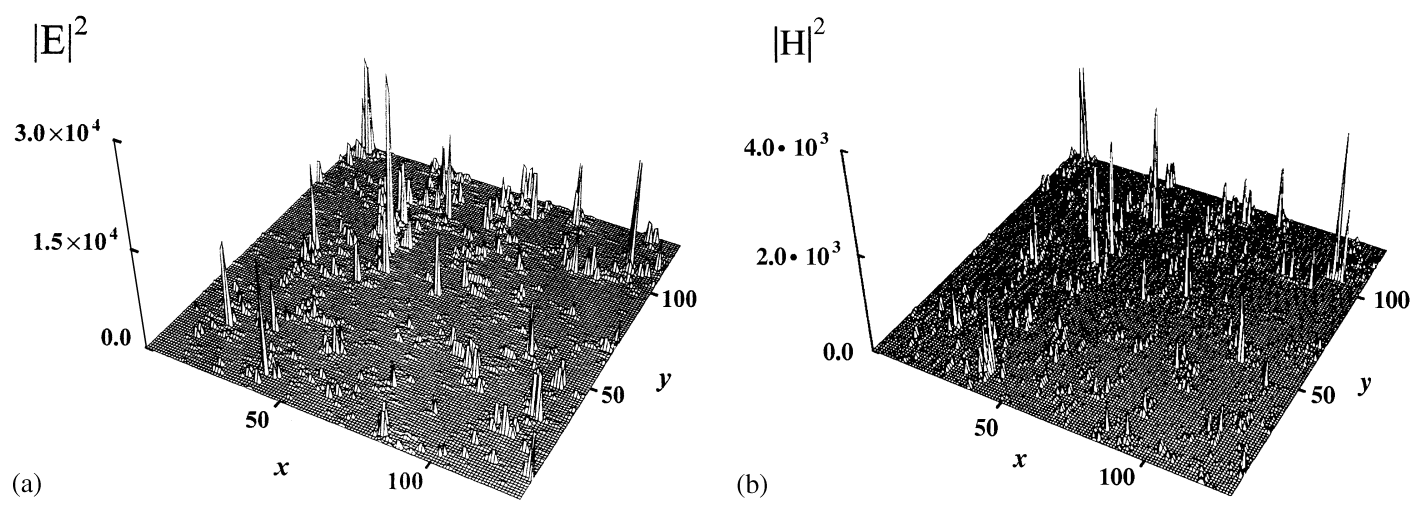

Fig. 25. Distribution of local em field intensities (a) $\left|\boldsymbol{E}_{1}(\boldsymbol{r})\right|^{2} /\left|\left\langle\boldsymbol{E}_{1}\right\rangle\right|^{2}$ and (b) $\left|\boldsymbol{H}_{1}(\boldsymbol{r})\right|^{2} /\left|\left\langle\boldsymbol{H}_{1}\right\rangle\right|^{2}$ in a semicontinuous silver film at the percolation threshold for $\lambda=1 \mu \mathrm{m}$ and $\delta / d=4.5$, where $\delta$ is the skin depth and $d$ is the thickness of the film. 

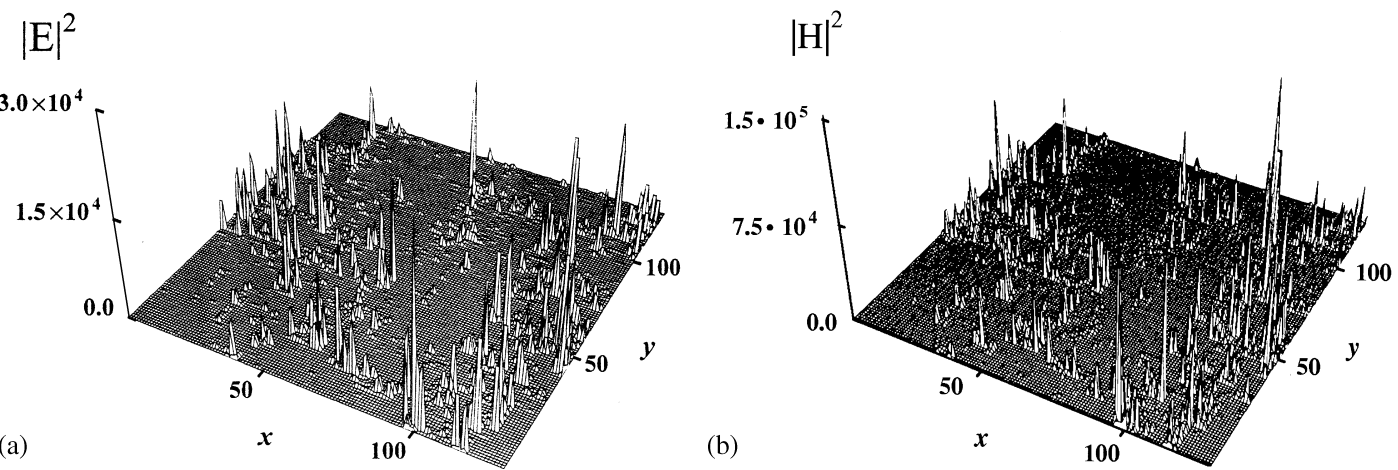

Fig. 26. Distribution of local em field intensities (a) $\left|\boldsymbol{E}_{1}(\boldsymbol{r})\right|^{2} /\left|\left\langle\boldsymbol{E}_{1}\right\rangle\right|^{2}$ and (b) $\left|\boldsymbol{H}_{1}(\boldsymbol{r})\right|^{2} /\left|\left\langle\boldsymbol{H}_{1}\right\rangle\right|^{2}$ in a semicontinuous silver film at the percolation threshold for $\lambda=1 \mu \mathrm{m}$ and $\delta / d=0.45$.
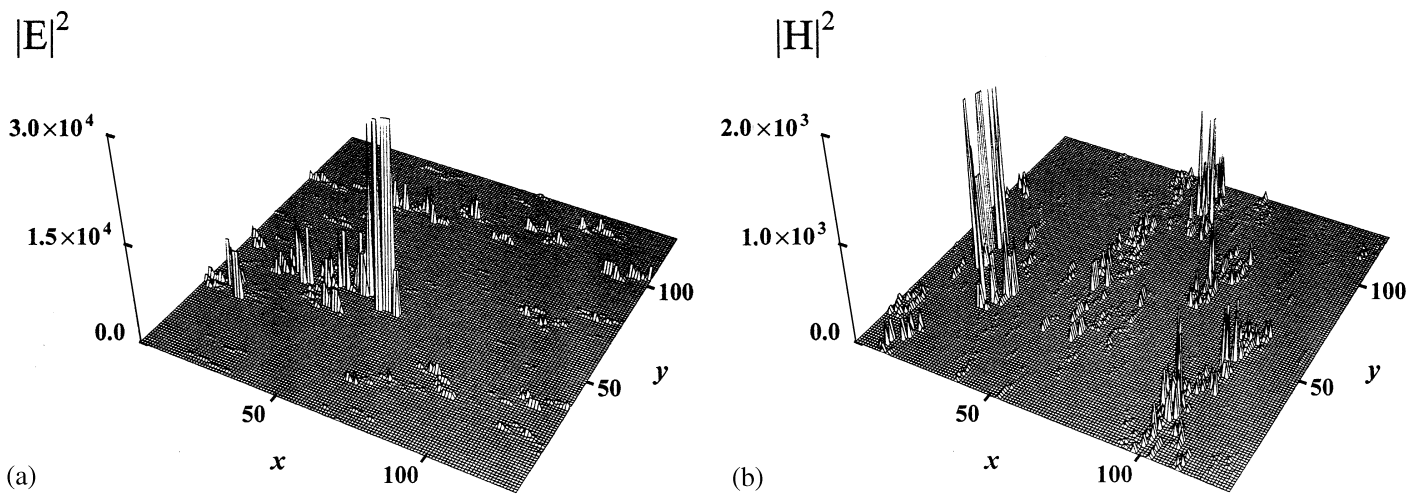

Fig. 27. Distribution of local em field intensities (a) $\left|\boldsymbol{E}_{1}(\boldsymbol{r})\right|^{2} /\left|\left\langle\boldsymbol{E}_{1}\right\rangle\right|^{2}$ and (b) $\left|\boldsymbol{H}_{1}(\boldsymbol{r})\right|^{2} /\left|\left\langle\boldsymbol{H}_{1}\right\rangle\right|^{2}$ in a semicontinuous silver film at the percolation threshold for $\lambda=10 \mu \mathrm{m}$ and $\delta / d=4.5$.
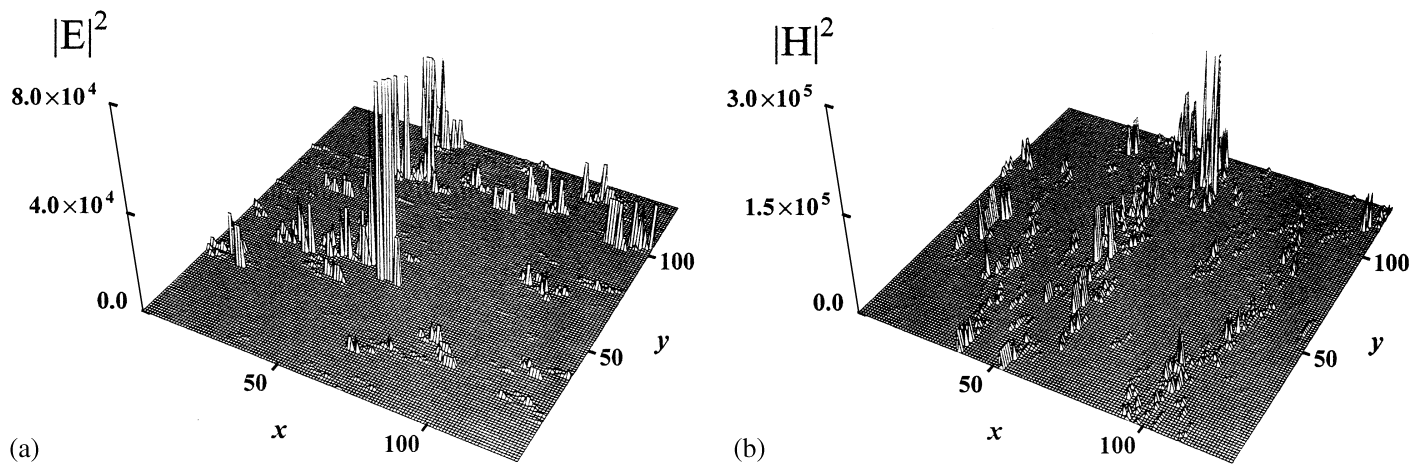

Fig. 28. Distribution of local em field intensities (a) $\left|\boldsymbol{E}_{1}(\boldsymbol{r})\right|^{2} /\left|\left\langle\boldsymbol{E}_{1}\right\rangle\right|^{2}$ and (b) $\left|\boldsymbol{H}_{1}(\boldsymbol{r})\right|^{2} /\left|\left\langle\boldsymbol{H}_{1}\right\rangle\right|^{2}$ in a semicontinuous silver film at the percolation threshold for $\lambda=10 \mu \mathrm{m}$ and $\delta / d=0.45$. 
(Fig. 25) corresponds to a weak skin effect since the dimensionless thickness is small, $\Delta \equiv d / \delta=0.2$ [where $\delta=1 /\left(k \operatorname{Im} n_{\mathrm{m}}\right)$ is the skin depth]. In this case we observe the giant field fluctuations of the local electric field; the magnetic field also strongly fluctuates over the film but the field peaks are small compared to the electric field. This is because the film itself is not magnetic, $\mu_{\mathrm{d}}=\mu_{\mathrm{m}}=1$, and the interaction of the magnetic field with the electric field through the skin effect is relatively small.

In Fig. 26 we show results for a significant skin effect, when the film thickness $d=50 \mathrm{~nm}$ and the dimensionless thickness exceeds one, $\Delta=2.2$. It is interesting to note that the amplitude of the electric field is roughly the same as in Fig. 25a, despite the fact that the parameter $\Delta$ increased by one order of magnitude. In contrast, the local magnetic field in Fig. 26b is strongly increased in this case so that the amplitude of magnetic field in peaks is of the same order of magnitude as the electric field maxima. This behavior can be understood by considering the spatial moments of the local magnetic field as shown in the next subsection.

In Figs. 27 and 28 we show results of the calculations for the local electric and magnetic fields at $\lambda=10 \mu \mathrm{m}$, when the metal dielectric constant $\left|\varepsilon_{\mathrm{m}}\right| \sim 10^{4}$. We see that in this case the local magnetic field can even exceed the electric field. It is interesting to note that the giant local field fluctuations were observed first in the microwave experiment [39] for the metal-dielectric films with strong skin effect. Later the local field peaks were obtained in the optic near field experiments $[46,58]$ as it is discussed in Section 3.2.

The local fields being given, the effective parameters $u_{\mathrm{e}}$ and $w_{\mathrm{e}}$ can be found and thus the effective optical properties of the film. In Figs. 29 and 30 we show the reflectance, transmittance and absorbance as functions of silver concentration $p$, for wavelengths $\lambda=1 \mu \mathrm{m}$ and $\lambda=10 \mu \mathrm{m}$ respectively. The absorbance in these figures has an anomalous maximum in the vicinity of the percolation threshold that corresponds to the behavior predicted by Eqs. (7.127). This maximum had been detected first in the experiments [85,88-90]. The maximum in the absorption corresponds to strong fluctuations of the local fields. We have estimated in Eqs. (7.126) the concentration range $\Delta p$ centered at the percolation threshold $p_{c}$ (where the giant local field fluctuations occur) as $\Delta p \propto 1 / \lambda$. Indeed, the absorbance shrinks at transition from Fig. 29 to Fig. 30, when wavelength $\lambda$ increases ten times. In Fig. 31 we compare the experimental results for gold semicontinuous film [85,88-90] with calculations based on the GOL approximation. In particular calculations the dynamic effective medium given by Eqs. (7.122) and (7.123) had been
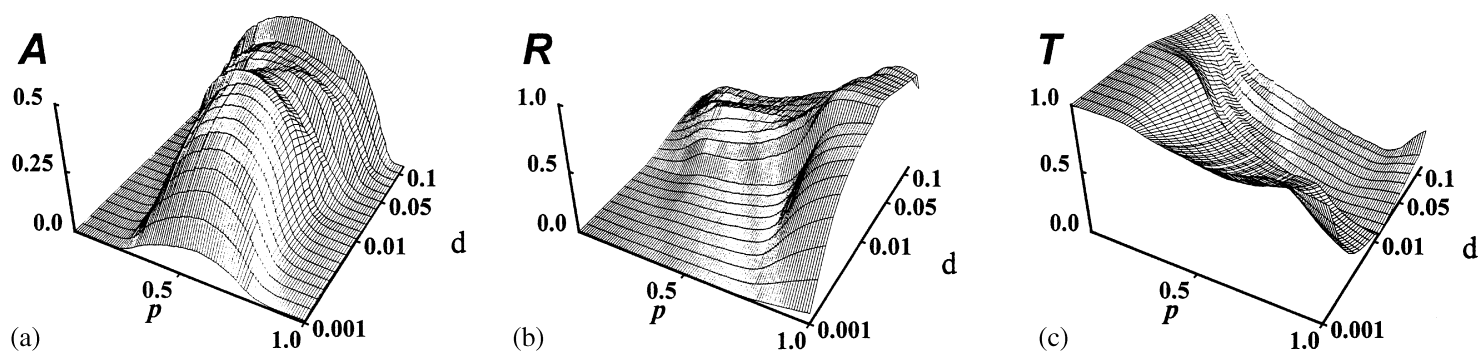

Fig. 29. Computer simulation of (a) absorptance $A$, (b) reflectance $R$ and (c) transmittance $T$ for a silver-on-glass semicontinuous film as functions of metal concentration $p$ and film thickness $d(\mu \mathrm{m})$ at $\lambda=1 \mu \mathrm{m}$. 

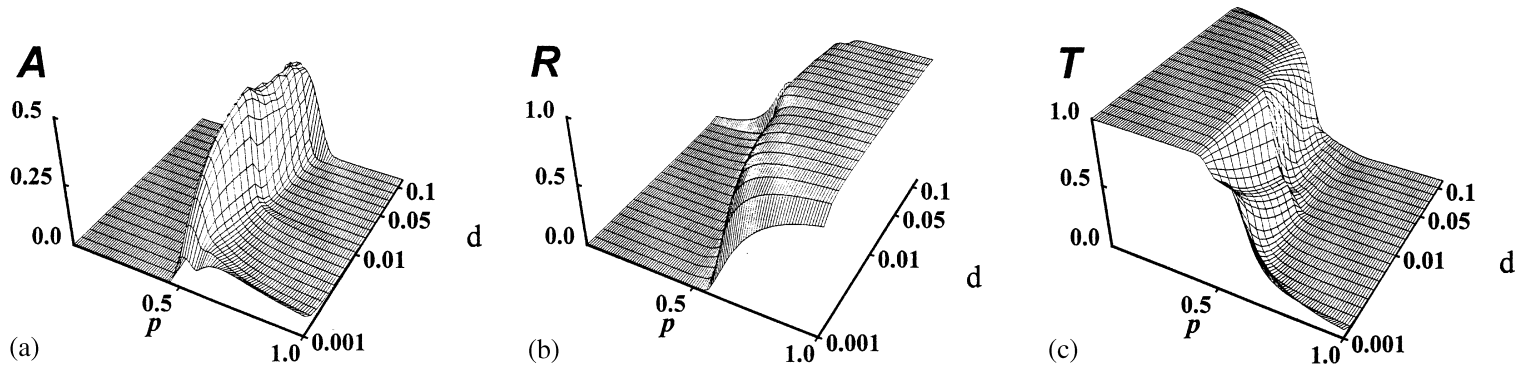

Fig. 30. Computer simulation of (a) absorptance $A$, (b) reflectance $R$ and (c) transmittance $T$ for a silver-on-glass semicontinuous film as functions of metal concentration $p$ and film thickness $d(\mu \mathrm{m})$ at $\lambda=10 \mu \mathrm{m}$.

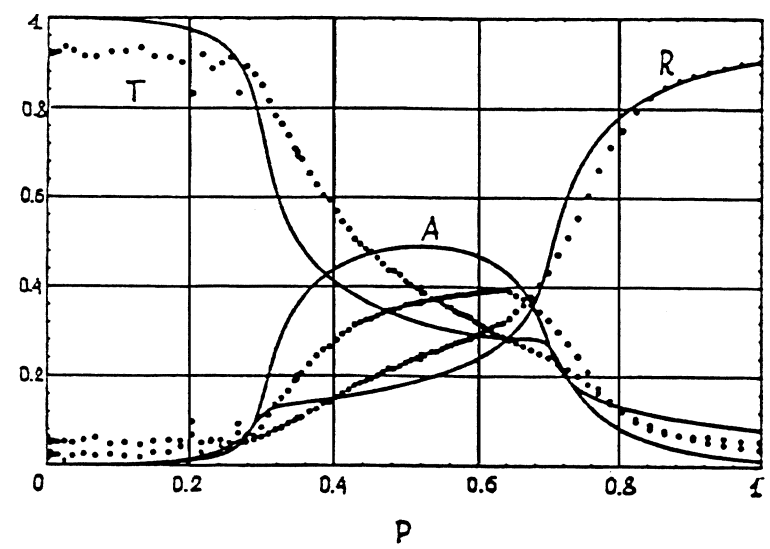

Fig. 31. Experimental and calculated coefficients $A$ (absorptance), $R$ (reflectance), and $T$ (transmittance) for a gold-glass film as functions of metal concetration $p$ at a wavelength $\lambda=2.2 \mu \mathrm{m}$. Solid circles show the experimental results [89,90], solid lines our calculations. The frequency-dependent complex conductivity of gold was taken to be $\sigma_{d c} /(1-\mathrm{i} \omega \tau)$, where $\sigma_{d c}=0.7 \times 10^{17} \mathrm{~s}^{-1}$ and $\tau=3 \times 10^{-15} \mathrm{~s}$, in accordance with Ref. [90]. The thickness of the gold grains was $d=20 \mathrm{~nm}$, and the value of the parameter $a$ was taken to be 3 .

used [96]. One observes that the theory indeed reproduces maximum of absorptance in the vicinity of the percolation threshold.

\subsubsection{Spatial moments of the local electric and magnetic fields}

The results obtained in the previous subsection allow to find spatial moments of the local electric $\boldsymbol{E}_{1}$ distributed in the reference plane $z=-d / 2-l_{0}$ (see Fig. 24) behind the film. The electric field $\boldsymbol{E}_{1}$ is expressed in terms of the fields $\boldsymbol{E}$ and $\boldsymbol{H}$ by means of Eq. (7.109). The fluctuations of the local magnetic current $\boldsymbol{j}_{H}(\boldsymbol{r})=w(\boldsymbol{r}) \boldsymbol{H}(\boldsymbol{r})$ can be neglected in the first Eq. (7.109) as it is discussed after Eq. (2.2). Therefore, the moments $M_{n, m}^{E}=\left\langle\left|\boldsymbol{E}_{1}(\boldsymbol{r})\right|^{n} \boldsymbol{E}_{1}^{m}(\boldsymbol{r})\right\rangle /\left(\left|\left\langle\boldsymbol{E}_{1}\right\rangle\right|^{n}\left\langle\boldsymbol{E}_{1}\right\rangle^{m}\right)$, where $\boldsymbol{E}_{1}^{m} \equiv\left(\boldsymbol{E}_{1} \cdot \boldsymbol{E}_{1}\right)^{m / 2}$, are approximately equal to the moments $M_{n, m}$ of the field $\boldsymbol{E}(\boldsymbol{r})$ estimated in Eq. (2.43). 
Consider the moments $M_{n, m}^{E}$ for arbitrarily strong skin effect assuming that the metal dielectric constant $\varepsilon_{\mathrm{m}}$ is negative, large in absolute value and can be approximated by Drude formula (2.1). The Drude formula Eq. (2.1) is substituted in Eq. (7.100) to obtain the Ohmic parameter $u_{\mathrm{m}}$ in the limit $\omega \ll \omega_{\mathrm{p}}$ and $\omega \gg \omega_{\tau}$; then the Ohmic parameter $u_{\mathrm{m}}$ is substituted in Eq. (7.128) to obtain $\tilde{\varepsilon}_{\mathrm{m}}$ and finally the moment $M_{n, m}^{E}$ is obtained from Eq. (2.43) as

$$
\begin{aligned}
& M_{n, m}^{E} \sim \rho\left[\frac{\omega_{\mathrm{p}}}{\omega_{\tau}}\left(\frac{a}{\xi_{\mathrm{A}}}\right)^{2} \sqrt{f_{0}(x)}\right]^{n+m-1}, \\
& f_{0}(x)=\frac{4 \tanh (x)^{3}\left[1+\frac{D}{4 \delta} \tanh (x)\right]}{x\left\{\tanh (x)+x\left[1-\tanh (x)^{2}\right]\right\}^{2}},
\end{aligned}
$$

where $x=d / 2 \delta=d \omega_{\mathrm{p}} / 2 c$ is the ratio of the film thickness $d$ to the skin depth $\delta \approx c / \omega_{\mathrm{p}}$. It follows from these equations that the moments of the local electric field are independent of the frequency in the wide frequency band $\omega_{\mathrm{p}} \gg \omega>\omega_{\tau}$ that include, for example, in the case of the silver semicontinuous films optical and infrared spectral ranges [cf. Fig. 5a]. When skin effect increases the function $f_{0}$ in Eq. (7.131) increases monotonically from $f_{0}(0)=1$ to $f_{0}(\infty)=D /(2 d)$. When the shape of the metal grains is fixed and they are very oblate, i.e., $D / d \gg 1$ the moments $M_{n, m}^{E}$ increases significantly with increasing the parameter $x$.

Let us consider now the far-infrared, microwave and radio frequency ranges, where the metal conductivity $\sigma_{\mathrm{m}}$ acquires its static value, i.e., it is positive and does not depend on frequency. Then it follows from Eqs. (7.103), (7.104) and (7.128) that Eq. (2.43) for the field moments acquires the following form:

$$
M_{n, m}^{E} \sim\left(\frac{2 \pi \sigma_{\mathrm{m}}}{\omega}\right)^{(n+m-1) / 2}
$$

in the limit of the strong skin effect. Since typical metal conductivity is much lager than frequency $\omega$ in the microwave and radio bands the moments remain large at these frequencies.

We proceed now to fluctuations of the local magnetic field $\boldsymbol{H}_{1}(\boldsymbol{r})$ in the reference plane $z=-d / 2-l_{0}$. The fluctuations of the field $\boldsymbol{H}(\boldsymbol{r})$ can still be neglected. Then it follows from the second Eq. (7.109) that moments $M_{n, m}^{H}=\left\langle\left|\boldsymbol{H}_{1}(\boldsymbol{r})\right|^{n} \boldsymbol{H}_{1}^{m}(\boldsymbol{r})\right\rangle /\left(\mid\left\langle\left.\boldsymbol{H}_{1}\right|^{n}\left\langle\boldsymbol{H}_{1}\right\rangle^{m}\right)\right.$ of the local magnetic field are estimated as

$$
M_{n, 0}^{H} \equiv M_{n}^{H} \simeq(2 \pi / c)^{n}\left\langle\left|\boldsymbol{j}_{E}(\boldsymbol{r})\right|^{n}\right\rangle /\left|\left\langle\boldsymbol{E}_{1}\right\rangle\right|^{n},
$$

where the conditions $\left|\left\langle\boldsymbol{E}_{1}\right\rangle\right|=\left|\left\langle\boldsymbol{H}_{1}\right\rangle\right|$, discussed in connection with Eq. (7.130), is used. Thus the external electric field induces electric currents in a semicontinuous metal film and these currents, in turn, generate the strongly fluctuating local magnetic field.

To estimate the moments $\left\langle\left|\boldsymbol{j}_{E}(\boldsymbol{r})\right|^{n}\right\rangle$ of the electric current density in semicontinuous metal films the approach suggested by Dykhne [100] was generalized for the nonlinear case [40]. Since in the considered case the electric current $\boldsymbol{j}_{E}$ is connected to the local field $\boldsymbol{E}$ via the first equation (7.98), the following equation $\left\langle\left|\boldsymbol{j}_{E}\right|^{n}\right\rangle=\alpha\left(u_{\mathrm{m}}, u_{\mathrm{d}}\right)\left\langle|\boldsymbol{E}(\boldsymbol{r})|^{n}\right\rangle$ can be written, where the coefficient $\alpha\left(u_{\mathrm{m}}, u_{\mathrm{d}}\right)$ is a function of variables $u_{\mathrm{m}}$ and $u_{\mathrm{d}}$.

Let us consider now the concentration corresponding to the percolation threshold $p=p_{c}$ and set the percolation value as $p_{c}=1 / 2$. It is also supposed that statistical properties of the system do not 
change when inter-replacing the metal and the dielectric. If all conductivities are increased by a factor $k$ then the average nonlinear current $\left\langle\left|\boldsymbol{j}_{E}\right|^{n}\right\rangle$ also increases by a factor $|k|^{n}$. Therefore, the coefficient $\alpha\left(u_{\mathrm{m}}, u_{\mathrm{d}}\right)$ increases by $|k|^{n}$ times as well. Then the coefficient $\alpha\left(u_{\mathrm{m}}, u_{\mathrm{d}}\right)$ has important scaling properties, namely, $\alpha\left(k u_{\mathrm{m}}, k u_{\mathrm{d}}\right)=|k|^{n} \alpha\left(u_{\mathrm{m}}, u_{\mathrm{d}}\right)$. By taking $k=1 / u_{\mathrm{m}}$ the following equation is obtained:

$$
\alpha\left(u_{\mathrm{m}}, u_{\mathrm{d}}\right)=\left|u_{\mathrm{m}}\right|^{n} \alpha_{1}\left(u_{\mathrm{m}} / u_{\mathrm{d}}\right) .
$$

Now we perform the Dykhne transformation

$$
\begin{aligned}
\boldsymbol{j}^{*} & =[\boldsymbol{n} \times \boldsymbol{E}], \\
\boldsymbol{E}^{*} & =\left[\boldsymbol{n} \times \boldsymbol{j}_{E}\right],
\end{aligned}
$$

It is easy to verify thus that the introduced field $\boldsymbol{E}^{*}$ is still potential, i.e., $\nabla \times \boldsymbol{E}^{*}=0$, whereas the current $\boldsymbol{j}^{*}$ is conserved, i.e., $\nabla \cdot \boldsymbol{j}^{*}=0$. The current $\boldsymbol{j}^{*}$ is coupled to the field $\boldsymbol{E}^{*}$ by the Ohm's law $\boldsymbol{j}^{*}=u^{*} \boldsymbol{E}^{*}$, where the "conductivity" $u^{*}$ takes values $1 / u_{\mathrm{m}}$ and $1 / u_{\mathrm{d}}$. Therefore, the following equation $\left\langle\left|\boldsymbol{j}^{*}\right|^{n}\right\rangle=\alpha\left(1 / u_{\mathrm{m}}, 1 / u_{\mathrm{d}}\right)\left\langle\left|\boldsymbol{E}^{*}\right|^{n}\right\rangle$ holds, from which it follows that $\alpha\left(1 / u_{\mathrm{m}}, 1 / u_{\mathrm{d}}\right) \alpha\left(u_{\mathrm{m}}, u_{\mathrm{d}}\right)=1$. Since we suppose that at the percolation threshold $p_{c}=1 / 2$ statistical properties of the system do not change when inter-replacing the metal and the dielectric the arguments in the first function can be changed to obtain $\alpha\left(1 / u_{\mathrm{d}}, 1 / u_{\mathrm{m}}\right) \alpha\left(u_{\mathrm{m}}, u_{\mathrm{d}}\right)=1$. This equation, in turn, can be rewritten using Eq. (7.135) as $\left|u_{\mathrm{m}} / u_{\mathrm{d}}\right|^{n} \alpha_{1}^{2}\left(u_{\mathrm{m}} / u_{\mathrm{d}}\right)=1$. Thus we find that $\alpha_{1}\left(u_{\mathrm{m}} / u_{\mathrm{d}}\right)=\left|u_{\mathrm{d}} / u_{\mathrm{m}}\right|^{n / 2}$, and the final result is given by $\alpha\left(u_{\mathrm{m}}, u_{\mathrm{d}}\right)=\left|u_{\mathrm{m}} u_{\mathrm{d}}\right|^{n / 2}$, i.e., the following generalization of the Dykhne's formula is valid:

$$
\left\langle\left|\boldsymbol{j}_{E}\right|^{n}\right\rangle=\left|u_{\mathrm{d}} u_{\mathrm{m}}\right|^{n / 2}\left\langle|\boldsymbol{E}|^{n}\right\rangle \text {. }
$$

This expression for $\left\langle\left|\boldsymbol{j}_{E}\right|^{n}\right\rangle$ is substituted in Eq. (7.134), which takes the following form:

$$
M_{n, m}^{H}=\left[\left(\frac{2 \pi}{c}\right)^{2}\left|u_{\mathrm{d}} u_{\mathrm{m}}\right|\right]^{n / 2} M_{n, m}^{E} .
$$

In optical and infrared spectral ranges it is possible to simplify this equation as it has been done for Eq. (7.134). Using again the Drude formula (2.1) for the metal dielectric constant and assuming that $\omega_{\tau} \ll \omega \ll \omega_{\mathbf{p}}$ the following estimate is obtained:

$$
M_{n, m}^{H}=\left[\varepsilon_{\mathrm{d}}^{\prime} \frac{x \tanh x}{(2 d / D)+x \tanh x}\right]^{n / 2} M_{n, m}^{E},
$$

where the moment $M_{n, m}^{E}$ is given by Eq. (7.134) and $x=d / 2 \delta=d \omega_{\mathrm{p}} / 2 c$ has the same meaning as in Eq. (7.134). It follows from Eq. (7.140) that spatial moments of the local magnetic field $M_{n, m}^{H}$ are of the same order of magnitude as moments of the local electric field $M_{n, m}^{E}$ in the limit of the strong skin effect, i.e., when $x \gg 1$.

We can estimate the moments of the local electric and magnetic fields from Eqs. (7.134) and (7.140) for silver-on-glass semicontinuous film with $\omega_{\mathfrak{p}}=9.1 \mathrm{eV}$, and $\omega_{\tau}=0.021 \mathrm{eV}$. Thus the moments of the local electric field are equal to $M_{n, m}^{E} \sim\left(4 \times 10^{2}\right)^{n-1}$, so that the field fluctuations are huge and they are in agreement with the numerical results shown in Figs. 25-28. For sufficient 


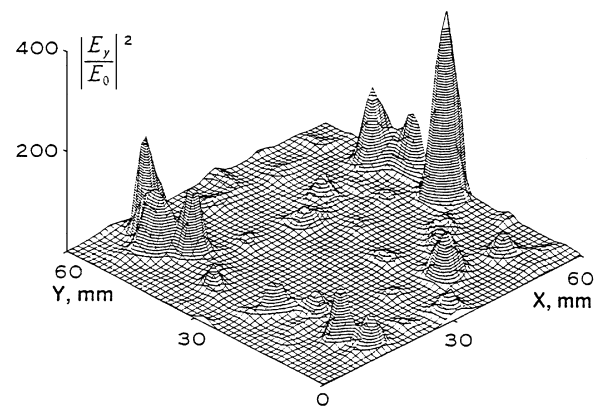

Fig. 32. Microwave localization. Electric field distribution in a macroscopic copper-dielectric film for $\lambda=2.5 \mathrm{~cm}$ at metal concentration $p=0.6$. Thickness of the copper spots $d=0.04 \mathrm{~mm}$.

strong skin effect $(x>1)$ the moments of local magnetic field $M_{n, m}^{H} \sim M_{n, m}^{E}$, which is also in agreement with computer results.

At frequencies much smaller than the relaxation rate $\omega_{\tau} \simeq 3.2 \times 10^{13} \mathrm{~s}^{-1}$ the silver conductivity acquires its static value $\omega_{\mathrm{p}}^{2} / 4 \pi \omega_{\tau} \simeq 10^{18} \mathrm{~s}^{-1}$. In this case the moments are given by Eq. (7.133). Thus for wavelength $\lambda=3 \mathrm{~cm}(\omega / 2 \pi=v=10 \mathrm{GHz})$ the moments are $M_{n, m}^{H} \sim M_{n, m}^{E} \sim\left(10^{4}\right)^{n}$. Thus we conclude that the local electric and magnetic field strongly fluctuate in a very large frequency range from the optical down to the microwave and radio spectral ranges. The fluctuations become even stronger for the microwave and radio bands. This is because for the strong skin effect (when the penetration depth is much smaller than the size of a metal grain), losses are small in comparison with the electromagnetic field energy accumulated around the film. This opens a fascinating possibility to observe the Anderson localization of the surface plasmons in microwave experiments with localization length in a centimeter scale.

Strong fluctuations of local electric field (see Fig. 32) was obtained in the microwave spectral range in the experiment [39]. In this experiment the local field was investigated in the macroscopic copper-dielectric films prepared by the lithographic method. The samples are composed of round spots made of copper foil and arranged on a plastic substrate. The diameter $a$ of the spots was given by $a=2 \mathrm{~mm}$ and thickness $d$ was given by $d=0.04 \mathrm{~mm}$. The microwave properties of the films were studied at the wavelength $\lambda=2.5 \mathrm{~cm}$ so that the skin depth $\delta \simeq 1.0 \mu \mathrm{m}$ was much less than the metal thickness $d \gg \delta$. In the case of regular (periodical) arrangement of the spots the absorptance $A$ was less than $A<5 \%$ for any surface concentration $p$ of the copper. For random arrangement of the spots a wide absorption band was observed around the percolation threshold $p_{c}$ where absorptance $A$ achieves $40 \%$. The local field fluctuations shown in Fig. 32 as well as effective absorptance in almost loss-free film allow us to speculate that the Anderson localization of the surface plasmons was observed in the experiment [39].

\section{Acknowledgements}

The studies described in this article were performed in close collaboration of authors with a number of excellent researchers, namely, Profs. Bergman, Boccara, Brouers, Clerc, Gadenne, 
Lagarkov, Rivoal, Drs. Antonov, Gresillon, Panina, Rosanov, Vinogradov, and Mr. Shubin. Useful discussions with Profs. Aharony, Boyd, Dykhne, Maradudin, Moskovits, Sheng, Soukoulis, Yablonovich, Ying, Drs. Safonov, Markel and Mr. Podolskiy are also highly appreciated. Work was supported in part by NSF (DMR-9810183), PRF, NATO, and RFFI (98-02-17628).

\section{References}

[1] L. de Arcangelis, S. Redner, H.J. Herrmann, J. Phys. Lett. 46 (1985) L585.

[2] P.M. Duxbury, P.L. Leath, P.D. Beale, Phys. Rev. B 36 (1987) 367.

[3] B. Khang, G.G. Bartrouni, S. Redner, L. de Arcangelis, H.J. Herrmann, Phys. Rev. B 37 (1988) 7625.

[4] A.P. Vinogradov, A.V. Goldenshtein, A.K. Sarychev, Zh. Tekh. Fiz. 59 (1989) 208 (English trans. in Sov. Phys. Tech. Phys. 34 (1989) 125); V.A. Garanov, A.A. Kalachev, A.M. Karimov, A.N. Lagarkov, S.M. Matytsin, A.B. Pakhomov, B.P. Peregood, A.K. Sarychev, A.P. Vinogradov, A.M. Virnic, J. Phys. 3 (1991) 3367; A.P. Vinogradov, V.A. Garanov, A.A. Kalachev, S.M. Matytsin, I.I. Oblakova, A.B. Pakhomov, A.K. Sarychev, Zh. Tekh. Fiz. 62 (1992) 44 (English trans. in Sov. Phys. Tech. Phys. (1992)).

[5] H.J. Herrmann, S. Roux (Eds.), Statistical Models for the Fracture of Disordered Media, Elsevier Science Publisher B.V. (North-Holland), Amsterdam, 1990; P. Meakin, Science 252 (1991) 226; P.M. Duxbury, P.L. Leath, Phys. Rev. B 49 (1994) 12,676; and references therein.

[6] K.K. Bardhan, R.K. Chakrabarty, Phys. Rev. Lett. 72 (1994); U.N. Nandi, K.K. Bardhan, Europhys. Lett. 31 (1995) 101; K.K. Bardhan, Physica A 241 (1997) 267.

[7] A.K. Sarychev, F. Brouers, Phys. Rev. Lett. 73 (1994) 2895.

[8] A. Aharony, Phys. Rev. Lett. 58 (1987) 2726.

[9] D. Stroud, P.M. Hui, Phys. Rev. B 37 (1988) 8719.

[10] V.M. Shalaev, M.I. Shtockman, Sov. Phys. JETP 65 (1987) 287 (trans. from Zh. Eksp. Teor. Fiz. 92 (1987) 509); A.V. Butenko, V.M. Shalaev, M.I. Shtockman, Sov. Phys. JETP 67 (1988) 60 (trans. from Zh. Eksp. Teor. Fiz. 94 (1988) 107).

[11] D.J. Bergman, Phys. Rev. B 39 (1989) 4598.

[12] D.J. Bergman, D. Stroud, Solid State Phys. 46 (1992) 14.

[13] P.M. Hui, in: K.K. Bardhan, B.K. Chakrabarty, A. Hansen (Eds.), Nonlinearity and Breakdown in Soft Condensed Matter, Lecture Notes in Physics, Vol. 437, Springer, Berlin, 1996.

[14] V.M. Shalaev, Phys. Rep. 272 (1996) 61.

[15] V.M. Shalaev, A.K. Sarychev, Phys. Rev. B 57 (1998) 13,265.

[16] Hongru Ma, Rongfu Xiao, Ping Sheng, J. Opt. Soc. Am. B 15 (1998) 1022.

[17] D. Stroud, Super-lattice Microstruct. 23 (1998) 567.

[18] D. Bergman, O. Levy, D. Stroud, Phys. Rev. B 49 (1994) 129; O. Levy, D. Bergman, Physica A 207 (1994) 157; O. Levy, D.J. Bergman, D.G. Stroud, Phys. Rev. E 52 (1995) 3184.

[19] R.W. Cohen, G.D. Cody, M.D. Coutts, B. Abeles, Phys. Rev. B 8 (1973) 3689.

[20] J.P. Clerc, G. Giraud, J.M. Luck, Adv. Phys. 39 (1990) 191.

[21] C. Flytzanis, Prog. Opt. 29 (1992) 2539 and references therein.

[22] V.M. Shalaev, M.I. Stockman, Sov. Phys. JETP 65 (1987) 287; A.V. Butenko, V.M. Shalaev, M.I. Stockman, Sov. Phys. JETP 67 (1988) 60; A.V. Butenko et al., Z. Phys. D. 17 (1990) 283; Yu.E. Danilova et al., S.G. Rautian, V.P. Safonov, P.A. Chubakov, V.M. Shalaev, M.I. Shtockman, JETP Lett. 47 (1988) 243 (trans. from Pis'ma Zh. Eksp. Teor. Fiz. 47 (1988) 200); Bull. Russian Acad. Sci. 60 (1996) 342; Yu.E. Danilova et al., S.G. Rautian, V.P. Safonov, P.A. Chubakov, V.M. Shalaev, M.I. Shtockman, 60 (1996) 374.

[23] V.M. Shalaev, M.I. Stockman, R. Botet, Physica A 185 (1992) 181; V.M. Shalaev, V.A. Markel, V.P. Safonov, Fractals 2 (1994) 201; V.M. Shalaev et al., Physica A 207 (1994) 197; V.M. Shalaev, E.Y. Poliakov, V.A. Markel, R. Botet, Physica A 241 (1997) 249.

[24] V.M. Shalaev, E.Y. Poliakov, V.A. Markel, Phys. Rev. B 53 (1996) 2437; V.A. Markel, V.M. Shalaev, E.B. Stechel, W. Kim, R.L. Armstrong, Phys. Rev. B 53 (1996) 2425. 
[25] M.I. Stockman, L.N. Pandey, L.S. Muratov, T.F. George, Phys. Rev. Lett. 72 (1994) 2486; M.I. Stockman, L.N. Pandey, L.S. Muratov, T.F. George, Phys. Rev. B 51 (1995); M.I. Stockman, L.N. Pandey, T.F. George, Phys. Rev. B 53 (1996) 2183.

[26] M.I. Stockman, Phys. Rev. Lett. 79 (1997) 4562; M.I. Stockman, Phys. Rev. E 56 (1997) 6494.

[27] V.M. Shalaev, R. Botet, A.V. Butenko, Phys. Rev. B 48 (1993) 6662; D.P. Tsai, J. Kovacs, Z. Wang, M. Moskovits, V.M. Shalaev, J. Suh, R. Botet, Phys. Rev. Lett. 72 (1994) 4149; V.M. Shalaev, M. Moskovits, Phys. Rev. Lett. 75 (1995) 2451; V.A. Markel, V.M. Shalaev, P. Zhang, W. Huynh, L. Tay, T.L. Haslett, M. Moskovits, Phys. Rev. Lett. 59 (1999) 10,903.

[28] V.M. Shalaev, R. Botet, J. Mercer, E.B. Stechel, Phys. Rev. B 54 (1996) 8235; E.Y. Poliakov, V.M. Shalaev, V.M. Markel, R. Botet, Opt. Lett. 21 (1996) 1628; V.M. Shalaev, C. Douketis, T. Haslett, T. Stuckless, M. Moskovits, Phys. Rev. B 53 (1996) 11,193; V.P. Safonov, V.M. Shalaev, V.A. Markel, Y.E. Danilova, N.N. Lepeshkin, W. Kim, S.G. Rautian, R.L. Armstrong, Phys. Rev. Lett. 80 (1998) 1102.

[29] G.L. Fisher, R.W. Boyd, R.J. Gehr, S.A. Jenekhe, J.A. Osaheni, J.E. Sipe, L.A. Weller-Brophy, Phys. Rev. Lett. 74 (1995) 1871; R.J. Gehr, G.L. Fisher, R.W. Boyd, J.E. Sipe, Phys. Rev. A 53 (1996) 2792; R.W. Boyd, R.J. Gehr, G.L. Fisher, J.E. Sipe, Pure Appl. Opt. 5 (1996) 505; and references therein.

[30] K.W. Yu, Phys. Rev. B 49 (1994) 9989.

[31] C. Zhang, X. Wu, S. Wu, Phys. Rev. B 54 (1996) 16,349.

[32] R. Rossignol, D. Ricard, K.C. Rustagi, Flytzanis, Opt. Commun. 55 (1985) 1413.

[33] G.R. Olbright, N. Peyghambarian, S.W. Koch, L. Banyai, Opt. Lett. 12 (1987) 413.

[34] D. Stroud, X. Zhang, Physica A 207 (1994) 55; X. Zhang, D. Stroud, Phys. Rev. B 49 (1994) 944.

[35] D.J. Bergman, E. Duering, M. Murat, J. Stat. Phys. 58 (1990) 1.

[36] F. Brouers, S. Blacher, A.K. Sarychev, Fractals in the Natural and Applied Sciences, Chapman \& Hall, London, 1995 (Chapter 24).

[37] F. Brouers, A.K. Sarychev, S. Blacher, O. Lothaire, Physica A 241 (1997) 146.

[38] F. Brouers, S. Blacher, A.N. Lagarkov, A.K. Sarychev, P. Gadenne, V.M. Shalaev, Phys. Rev. B 55 (1997) $13,234$.

[39] A.N. Lagarkov, K.N. Rozanov, A.K. Sarychev, A.N. Simonov, Physica A 241 (1997) 199.

[40] E.M. Baskin, M.V. Entin, A.K. Sarychev, A.A. Snarskii, Physica A 242 (1997) 49.

[41] V.M. Shalaev, E.Y. Poliakov, V.A. Markel, V.P. Safonov, A.K. Sarychev, Fractals 5 (1997) 63.

[42] P. Gadenne, F. Brouers, V.M. Shalaev, A.K. Sarychev, J. Opt. Soc. Am. B 15 (1998) 68.

[43] V.M. Shalaev, V.A. Markel, E.Y. Poliakov, R.L. Armstrong, V.P. Safonov, A.K. Sarychev, J. Nonlinear Opt. Phys. Mater. 7 (1998) 131.

[44] F. Brouers, S. Blacher, A.K. Sarychev, Phys. Rev. B 58 (1998) 15,897.

[45] A.K. Sarychev, V.A. Shubin, V.M. Shalaev, Phys. Rev. E 59 (1999) 7239.

[46] S. Grésillon, L. Aigouy, A.C. Boccara, J.C. Rivoal, X. Quelin, C. Desmarest, P. Gadenne, V.A. Shubin, A.K. Sarychev, V.M. Shalaev, Phys. Rev. Lett. 82 (1999) 4520.

[47] A.K. Sarychev, V.A. Shubin, V.M. Shalaev, Physica A 266 (1999) 115.

[48] A.K. Sarychev, V.A. Shubin, V.M. Shalaev, Phys. Rev. B 60 (1999) 23.

[49] D.A.G. Bruggeman, Ann. Phys. (Leipzig) 24 (1935) 636.

[50] X.C. Zeng, D.J. Bergman, P.M. Hui, D. Stroud, Phys. Rev. B 38 (1988) 10,970; X.C. Zeng, P.M. Hui, D.J. Bergman, D. Stroud, Physica A 157 (1989) 10,970.

[51] D.J. Bergman, in: G. Dal Maso, G.F. Dell'Antinio (Eds.), Composite Media and Homogenization Theory, Brikhauser, Boston, 1991, p. 67.

[52] O. Levy, D.J. Bergman, J. Phys. C: Condens. Matter 5 (1993) 7095.

[53] H.C. Lee, K.P. Yuen, K.W. Yu, Phys. Rev. B 51 (1995) 9317.

[54] W.M.V. Wan, H.C. Lee, P.M. Hui, K.W. Yu, Phys. Rev. B 54 (1996) 3946.

[55] P.M. Hui, P. Cheung, Y.R. Kwong, Physica A 241 (1997) 301, and references therein.

[56] P.M. Hui, D. Stroud, Phys. Rev. B 49 (1994) 11,729.

[57] G.W. Milton, Appl. Phys. A 26 (1981) 1207; G.W. Milton, J. Appl. Phys. 52 (1980) 5286.

[58] S. Gresillon, J.C. Rivoal, P. Gadenne, X. Quelin, V.M. Shalaev, A.K. Sarychev, Phys. Stat. Sol. A 175 (1999) 337.

[59] S.I. Bozhevolnyi, I.I. Smolyaninov, A.V. Zayats, Phys. Rev. B 51 (1995) 17,916.

[60] S.I. Bozhevolnyi, V.A. Markel, V. Coello, W. Kim, V.M. Shalaev, Phys. Rev. B 58 (1998) 11,441. 
[61] Ping Sheng, Introduction to Wave Scattering, Localization, and Mesoscopic Phenomena, Academic Press, San Diego, 1995.

[62] D.F. Sievenpiper, M.E. Sickmiller, E. Yablonovitch, Phys. Rev. Lett. 76 (1996) 2480.

[63] J.B. Pendry, A.J. Holden, W.J. Stewart, I. Youngs, Phys. Rev. Lett. 76 (1996) 4773.

[64] D. Stauffer, A. Aharony, An Introduction to Percolation Theory, 2nd Edition, Taylor and Francis, London, 1994.

[65] H.E. Stanley, Introduction to Phase Transition and Critical Phenomena, Oxford Press, Oxford, 1981; I.L. Fabelinskii, Molecular Scattering of Light, Plenum, NY, 1968.

[66] P.M. Chaikin, T.C. Lubensky, Principles of Condensed Matter Physics, Cambridge University Press, Cambridge, 1995.

[67] A. Aharony, R. Blumenfeld, A.B. Harris, Phys. Rev. B 47 (1993) 5756.

[68] B. Kramer, A. MacKinnon, Rep. Prog. Phys. 56 (1993) 1469.

[69] D. Belitz, T.R. Kirkpatrick, Rev. Mod. Phys. 66 (1994) 261.

[70] M.V. Sadovskii, Phys. Rep. 282 (1997) 225.

[71] K.B. Efetov, Supersymmetry in Disorder and Chaos, Cambridge University Press, Cambridge, UK, 1997.

[72] J.A. Verges, Phys. Rev. B 57 (1998) 870.

[73] A. Elimes, R.A. Romer, M. Schreiber, Eur. Phys. J. B 1 (1998) 29.

[74] T. Kawarabayashi, B. Kramer, T. Ohtsuki, Phys. Rev. B 57 (1998) 11,842.

[75] V.I. Fal'ko, K.B. Efetov, Phys. Rev. B 52 (1995) 17,413.

[76] K. Muller et al., Phys. Rev. Lett. 78 (1997) 215.

[77] M.V. Berry, J. Phys. A 10 (1977) 2083.

[78] A.V. Andreev et al., Phys. Rev. Lett. 76 (1996) 3947.

[79] M. Kaveh, N.F. Mott, J. Phys. A 14 (1981) 259.

[80] I.E. Smolyarenko, B.L. Altshuler, Phys. Rev. B 55 (1997) 10,451.

[81] R.W. Boyd, Nonlinear Optics, Academic Press, New York, 1992.

[82] L.D. Landau, E.M. Lifshits, L.P. Pitaevskii, Electromagnetics of Continuous Media, 2nd Edition, Pergamon, Oxford, 1984.

[83] P.J. Reynolds, W. Klein, H.E. Stanley, J. Phys. C 10 (1977) L167; A.K. Sarychev, Sov. Phys. JETP 45 (1977) 524.

[84] G.A. Niklasson, C.G. Granquist, J. Appl. Phys. 55 (1984) 3382.

[85] Y. Yagil, P. Gadenne, C. Julien, G. Deutscher, Phys. Rev. 46 (1992) 2503.

[86] T.W. Noh, P.H. Song, Sung-Il Lee, D.C. Harris, J.R. Gaines, J.C. Garland, Phys. Rev. 46 (1992) 4212.

[87] F. Brouers, J.P. Clerc, G. Giraud, Phys. Rev. B 44 (1991) 5299.

[88] P. Gadenne, A. Beghadi, J. Lafait, Opt. Commun. 65 (1988) 17.

[89] P. Gadenne, Y. Yagil, G. Deutscher, J. Appl. Phys. 66 (1989) 3019.

[90] Y. Yagil, M. Yosefin, D.J. Bergman, G. Deutscher, P. Gadenne, Phys. Rev. B 43 (1991) 11,342.

[91] L.C. Botten, R.C. McPhedran, Opt. Acta 32 (1985) 595; M. Gajdardziska-Josifovska, R.C. McPhedran, D.R. McKenzie, R.E. Collins, Appl. Opt. 28 (1989) 2744; C.A. Davis, D.R. McKenzie, R.C. McPhedran, Opt. Commun. 85 (1991) 70.

[92] J.C. Maxwell Garnett, Philos. Trans. Roy. Soc. London 203 (1904) 385.

[93] A.P. Vinogradov, A.M. Karimov, A.K. Sarychev, Zh. Eksp. Teor. Fiz. 94 (1988) 301 (Sov. Phys. JETP 67 (1988) 2129).

[94] G. Depardieu, P. Frioni, S. Berthier, Physica A 207 (1994) 110.

[95] A.K. Sarychev, D.J. Bergman, Y. Yagil, Physica A 207 (1994) 372.

[96] A.K. Sarychev, D.J. Bergman, Y. Yagil, Phys. Rev. B 51 (1995) 5366.

[97] Levy-Nathansohn, D.J. Bergman, Physica A 241 (1997) 166; Phys. Rev. B 55 (1997) 5425.

[98] X.C. Zeng, P.M. Hui, D. Stroud, Phys. Rev. B 39 (1989) 1063.

[99] F. Brouers, J.P. Clerc, G. Giraud, Phys. Rev. B 47 (1993) 666.

[100] A.M. Dykhne, Zh. Eksp. Teor. Fiz. 59 (1970) 110 (Sov. Phys. JETP 32 (1971) 348).

[101] J. Bernasconi, Phys. Rev. B 18 (1978) 2185.

[102] A. Aharony, Physica A 205 (1994) 330.

[103] D.J. Frank, C.J. Lobb, Phys. Rev. B 37 (1988) 302.

[104] E.D. Palik (Ed.), Hand book of Optical Constants of Solids, Academic Press, New York, 1985. 
[105] V.M. Shalaev, R. Botet, R. Jullien, Phys. Rev. B 44 (1991) 12,216.

[106] B. Hesselbo, D. Phil. Thesis, Oxford University, 1994.

[107] A.N. Lagarkov, L.V. Panina, A.K., A.K. Sarychev, Y.R. Smychkovich, A.P. Vinogradov, MRS Symposium Proceedings Vol. 195, Pittsburgh, 1990, p. 275; A.N. Lagarkov, A.K. Sarychev, Y.R. Smychkovich, A.P. Vinogradov, J. Electromag. Waves Appl. 6 (1992) 1159.

[108] See, e.g., D.J. Semin, A. Lo, S.E. Roak, R.T. Skodje, K.L. Rowlen, J. Chem. Phys. 105 (1996) 5542.

[109] M. Moskovits, Rev. Mod. Phys. 57 (1985) 783.

[110] K. Kneipp, Y. Wang, H. Kneipp, Lt. Perelman, I. Itzkan, R. Dasari, Ms. Feld, Phys. Rev. Lett. 78 (1997) 1667.

[111] S. Nie, S.R. Emory, Science 275 (1997) 1102.

[112] L.A. Lyon, C.D. Keating, A.P. Fox, B.E. Baker, L. He, S.R. Nicewarner, S.P. Mulvaney, M.J. Natan, Anal. Chem. 70 (1998) R341.

[113] R.K. Chang, T.E. Furtak (Eds.), Surface Enhance Raman Scattering, Plenum Press, New York, 1982.

[114] See, e.g., M.A. Tadayoni, N.R. Dando, Appl. Spectrosc. 45 (1991) 1613.

[115] A. Liebsch, W.L. Schaich, Phys. Rev. B 40 (1989) 5401.

[116] R. Murphy, M. Yeganeh, K.J. Song, E.W. Plummer, Phys. Rev. Lett. 63 (1989) 318.

[117] G.A. Reider, T.F. Heinz, in: P. Halevi (Ed.), Photonic Probes of Surfaces: Electromagnetic Waves, Elsevier, Amsterdam, 1995.

[118] T.Y.F. Tsang, Phys. Rev. A 52 (1995) 4116.

[119] R.A. Watts, T.W. Preist, J.R. Sambles, Phys. Rev. Lett. 79 (1997) 3978.

[120] A.R. McGurn, A.A. Maradudin, V. Celli, Phys. Rev. B 31 (1985) 4866; A.R. McGurn, Surf. Sci. Rep. 10 (1990) 359.

[121] J.A. Sanchez-Gil, A.A. Maradudin, J.Q. Lu, V.D. Freilikher, M. Pustilnik, I. Yurkevich, Rev. B 50 (1994) $15,353$.

[122] T.Y.F. Tsang, Opt. Lett. 21 (1996) 245.

[123] B. Hecht, H. Bielefeldt, L. Novotny, Y. Inouye, D.W. Pohl, Phys. Rev. Lett. 77 (1996) 1889.

[124] O.A. Aktsipetrov, O. Keller, K. Pedersen, A.A. Nikulin, N.N. Novikova, A.A. Fedyanin, Phys. Lett. A 179 (1993) 149.

[125] L. Kuang, H.J. Simon, Phys. Lett. A 197 (1995) 257.

[126] A.N. Lagarkov, L.V. Panina, A.K. Sarychev, Zh. Eksp. Teor. Fiz. 93 (1987) 215 (Sov. Phys. JETP 66 (1987) 123).

[127] A.P. Vinogradov, L.V. Panina, A.K. Sarychev, Dokl. Akad. Nauk SSSR 306 (1989) 847.

[128] D. Rousselle, A. Berthault, O. Acher, J.P. Bouchaud, P.G. Zérah, J. Appl. Phys. 74 (1993) 475.

[129] L.V. Panina, A.S. Antonov, A.K. Sarychev, V.P. Paramonov, E.V. Timasheva, J. Appl. Phys. 76 (1994) 6365.

[130] J.D. Jackson, Classical Electrodynamics, 3rd Edition, Wiley, New York, 1998.

[131] N.A. Nicorovici, R.C. McPhedran, L.C. Botten, Phys. Rev. Lett. 75 (1995) 1507; Phys. Rev. E 52 (1995) 1135.

[132] R.C. McPhedran, N.A. Nicorovici, L.C. Botten, J. Electromagn. Waves Appl. 11 (1997) 981.

[133] A.N. Lagarkov, A.K. Sarychev, Phys. Rev. B 53 (1996) 6318.

[134] A.N. Lagarkov, S.M. Matytsin, K.N. Rozanov, A.K. Sarychev, Physica A 241 (1997) 58.

[135] A.N. Lagarkov, S.M. Matytsin, K.N. Rozanov, A.K. Sarychev, J. Appl. Phys. 84 (1998) 3806.

[136] M. Golosovsky, M. Tsindlekht, D. Davidov, Phys. Rev. B 46 (1992) 11,439; M. Golosovsky, M. Tsindlekht, D. Davidov, A.K. Sarychev, Physica C 209 (1993) 337.

[137] V.A. Markel, T.F. George (Eds.), Optics of Nanostructured Materials, Wiley, New York, 2000 (tentatively).

[138] A.A. Kalachev, S.M. Matitsin, K.N. Rosanov, A.K. Sarychev, Method for measuring the complex dielectric constant of sheet materials, USSR Patent No. 1483394, 1987 (USSR Bull. Izobr. Otkr. No. 20, 1989).

[139] A.A. Kalachev, I.V. Kukolev, S.M. Matitsin, L.N. Novogrudskiy, K.N. Rosanov, A.K. Sarychev, A.V. Selesnev, in: J.A. Emerson, J.M. Torkelson (Eds.), Optical and Electrical Properties of Polymers, MRS 214, Pittsburg, 1991.

[140] H.B. Liao et al., Appl. Phys. Lett. 72 (1998) 1817. 